\title{
Taken Out of Context \\ American Teen Sociality in Networked Publics
}

\author{
by \\ danah michele boyd \\ B.A. (Brown University) 2001 \\ M.S. (Massachusetts Institute of Technology) 2002
}

A dissertation submitted in partial satisfaction of the requirements for the degree of

\author{
Doctor of Philosophy \\ in \\ Information Management and Systems \\ and the Designated Emphasis \\ in \\ New Media \\ in the \\ Graduate Division \\ of the \\ University of California, Berkeley
}

Committee in charge:

Professor AnnaLee Saxenian, Chair

Doctor Mizuko Ito

Professor Cori Hayden

Professor Jenna Burrell

Fall 2008 
Taken Out of Context: American Teen Sociality in Networked Publics

Copyright (C) 2008, Some Rights Reserved (See: Appendix 3)

danah michele boyd 


\begin{abstract}
Taken Out of Context: American Teen Sociality in Networked Publics

by

danah michele boyd

Doctor of Philosophy in Information Management and Systems

with a Designated Emphasis in New Media

University of California, Berkeley

Professor AnnaLee Saxenian, Chair
\end{abstract}

As social network sites like MySpace and Facebook emerged, American teenagers began adopting them as spaces to mark identity and socialize with peers. Teens leveraged these sites for a wide array of everyday social practices-gossiping, flirting, joking around, sharing information, and simply hanging out. While social network sites were predominantly used by teens as a peer-based social outlet, the unchartered nature of these sites generated fear among adults. This dissertation documents my 2.5-year ethnographic study of American teens' engagement with social network sites and the ways in which their participation supported and complicated three practices-self-presentation, peer sociality, and negotiating adult society.

My analysis centers on how social network sites can be understood as networked publics which are simultaneously (1) the space constructed through networked 
technologies and (2) the imagined community that emerges as a result of the intersection of people, technology, and practice. Networked publics support many of the same practices as unmediated publics, but their structural differences often inflect practices in unique ways. Four properties-persistence, searchability, replicability, and scalability-and three dynamics-invisible audiences, collapsed contexts, and the blurring of public and private-are examined and woven throughout the discussion.

While teenagers primarily leverage social network sites to engage in common practices, the properties of these sites configured their practices and teens were forced to contend with the resultant dynamics. Often, in doing so, they reworked the technology for their purposes. As teenagers learned to navigate social network sites, they developed potent strategies for managing the complexities of and social awkwardness incurred by these sites. Their strategies reveal how new forms of social media are incorporated into everyday life, complicating some practices and reinforcing others. New technologies reshape public life, but teens' engagement also reconfigures the technology itself. 
This dissertation is dedicated to my beloved advisor, Peter Lyman (1940-2007), for all of the support, guidance, and love he provided along the way. I could not have asked for a better friend, mentor, and partner in crime. May he rest in peace knowing that I finally finished. 


\section{Table of Contents}

Chapter 1: Introduction 1

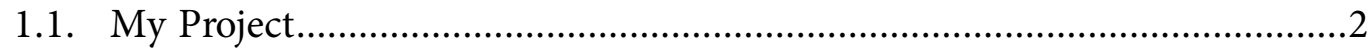

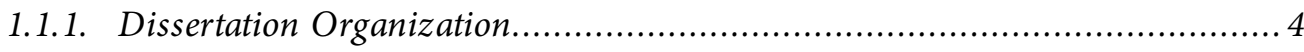

1.2. The Social Construction of Teenagers..........................................................6

1.3. Technology and Change............................................................................11

1.4. Locating Networked Publics........................................................................15

1.4.1. Public and Publics............................................................................. 16

1.4.2. Teenagers and Publics ......................................................................... 21

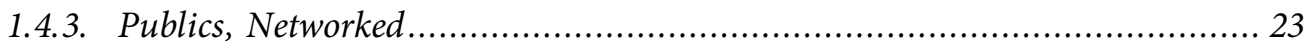

1.5. The Structure of Networked Publics.............................................................26

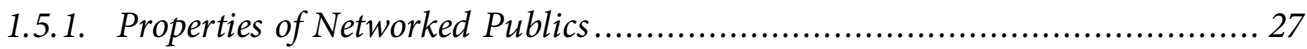

1.5.2. New Dynamics Resulting from Networked Publics .................................... 34

Chapter 2: Choose Your Own Ethnography 42

2.1. Ethnography in Context .........................................................................46

2.1.1. Ethnography and the Internet............................................................. 48

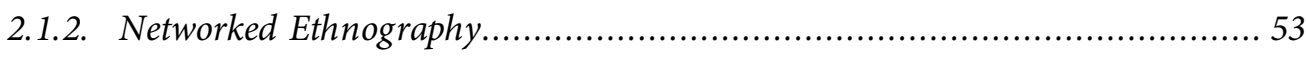

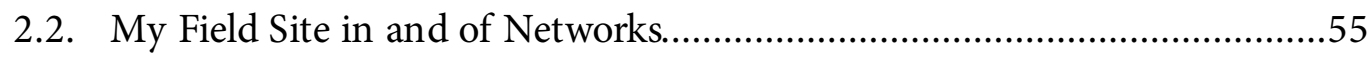

2.2.1. From Youth to American Teenagers ........................................................ 56

2.2.2. From Networked Publics to MySpace and Facebook .......................................... 61

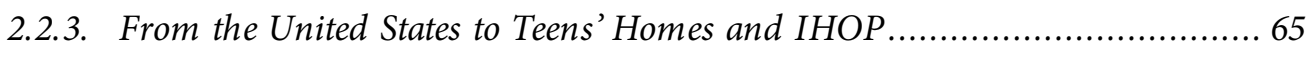

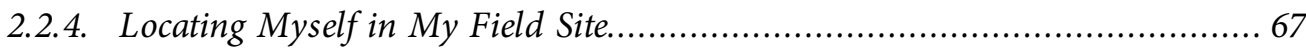

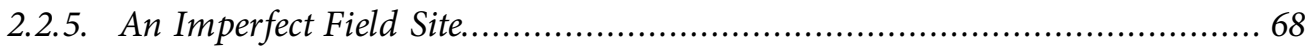

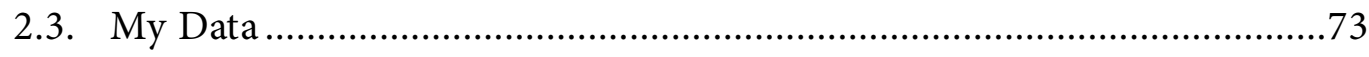

2.3.1. Online Data and Observation ........................................................... 74

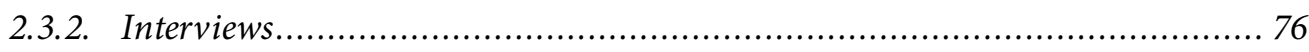


2.3.3. Fieldwork in Less Structured Environments. 80

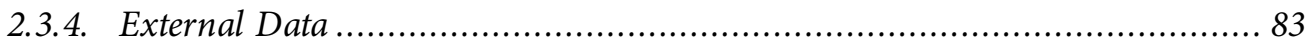

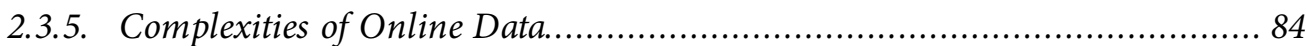

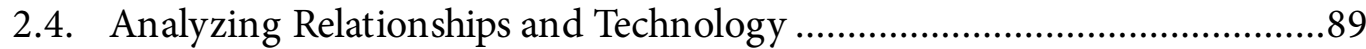

Chapter 3: Social Network Sites and Social Media $\quad 92$

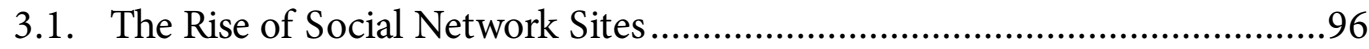

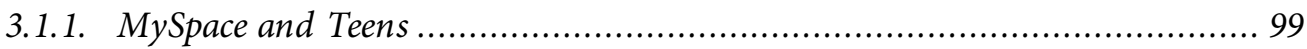

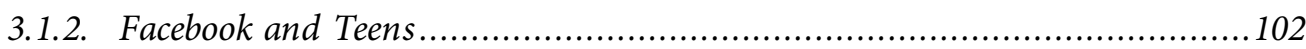

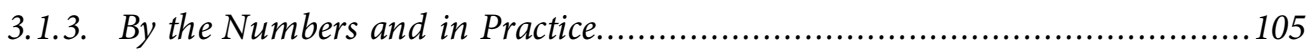

3.2. Participation in Context .....................................................................107

3.2.1. Negotiating Multiple Communication Channels...................................110

3.2.2. Teen vs. Adult Social Media Practices...................................................114

Chapter 4: Writing Oneself into Being 119

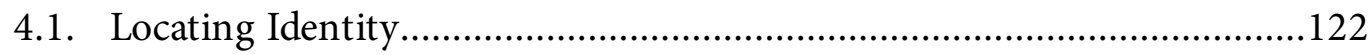

4.2. Writing Identity into Being Online.................................................126

4.3. The Art of Profile Creation and Management........................................129

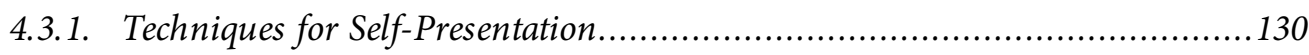

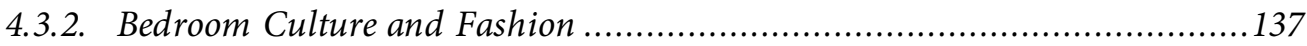

4.3.3. Varying Degrees of Participation .................................................... 140

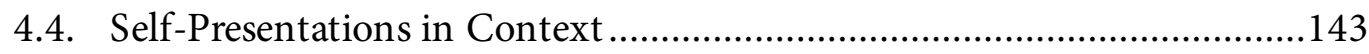

4.5. Performing Falsehoods-Deception, Play, or Control? ..........................148

4.5.1. Motivations for Providing Inaccurate Information................................ 149

4.5.2. Legal and Technical Limitations ................................................... 151

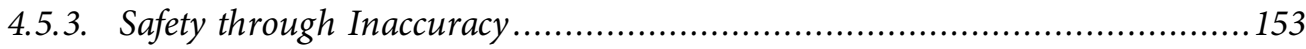

4.6. Controlling Access: Public or Private? .................................................159

4.7. Managing Identity in Networked Publics...........................................167 
5.1. Peer Relations and Teen Friendship.............................................................173

5.2. Pressure to Participate: Signing Up and Opting Out ................................176

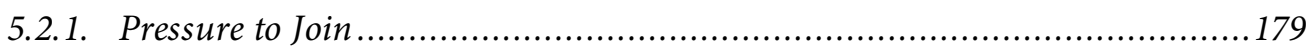

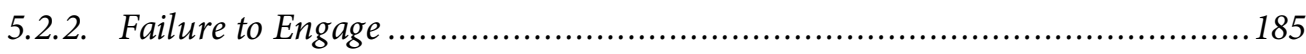

5.3. MySpace vs. Facebook: Social Categories and Networked Turf..................196

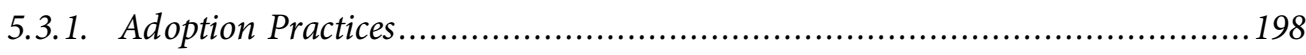

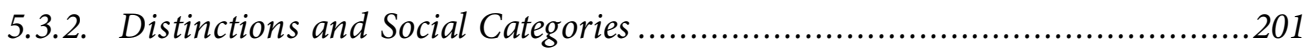

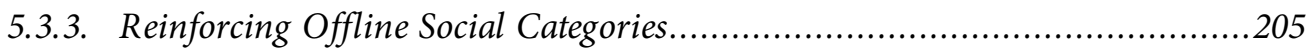

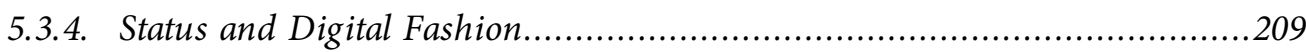

5.4. Public Displays of Connection and Status .................................................212

5.4.1. Strategies for Friending ....................................................................213

5.4.2. Hierarchies of Friends.......................................................................220

5.5. Status Battles and Peer Dramas.................................................................227

5.6. Peer Sociality in Networked Publics.........................................................237

Chapter 6: Power and Control $\quad 241$

6.1. Social and Structural Controls .................................................................245

6.2. Contemporary Adult-Teen Dynamics.......................................................249

6.2.1. Household Dynamics .....................................................................249

6.2.2. Engaging with Teachers, Youth Pastors, and Other Trusted Adults.............257

6.3. Fears and Moral Panics..........................................................................262

6.3.1. The MySpace Moral Panic....................................................................264

6.3.2. Teen Responses to a Culture of Fear ........................................................267

6.4. Access, Privacy, and Control..................................................................275

6.4.1. Restricting Access and Mobility .......................................................277

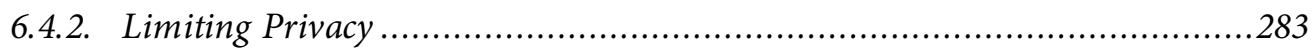

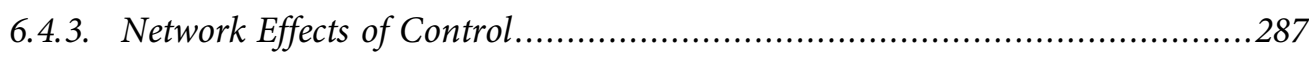

6.5. In the Pursuit of Freedom .......................................................................290 
7.1. Lessons from the Everyday Lives of Teens ...............................................293

7.2. The Significance of Publics........................................................................297

7.3. The Future of Networked Publics ...............................................................300

Bibliography $\quad 304$

Appendix 1: Brief Descriptions of Teens 337

Appendix 2: Features and Functionality $\quad 376$

Appendix 3: Creative Commons License $\quad 385$ 


\section{Acknowledgments}

“Taken out of context, I must seem so strange..."-Ani DiFranco, Fire Door

While writing a dissertation is one of the most isolating activities imaginable, I was never truly alone. I had the constant support and encouragement of family, friends, colleagues, and mentors. I cannot even begin to express my gratitude for their willingness to put up with me during these years.

The love and support that I received from my partner Gilad during this process exceeded my wildest dreams. He lived with me throughout this process and bore the brunt of my exhaustion, confusion, and frustration. Without complaint, he responded with chicken noodle soup and hugs. On my most recent birthday, he surprised me with a party and a cake. When I asked why the number of candles did not equal my years since birth, he explained that he had subtracted my time in graduate school. Gilad's love, humor, and patience made it possible for me to keep going and, for that, I am eternally grateful to him.

I am tremendously thankful for all of my family and their love. I am especially appreciative of my brother Ryan's willingness to be there for me even when I was most crazy. And to my grandparents who have embraced my insanity as proof that I am family; their willingness to go for an adventure and attend my graduation prompted me to work harder and faster. Finally, I would not be who I am today 
without my mother Kathryn. Mom-this document is the answer to your persistent question, “Are you done yet?” Finally, w00t!

When friends ask about my dissertation committee, I refer to Mimi Ito, Cori Hayden, Jenna Burrell, and Anno Saxenian as "the goddesses." I have had the great fortune of working with four brilliant women who have guided me and shaped me with boundless patience and encouragement. Their intellectual engagement with my ideas and support of my endeavors is what made this process worth it.

I have been surrounded by a great group of scholars, researchers, and thinkers who have helped me stay grounded. Ronen Barzel, Elizabeth Churchill, Cory Doctorow, Liz Goodman, Jo Guldi, Hannah Rohde, Irina Shklovski, and Wendy Ju, have helped me find humor in this process while always being there with an understanding shoulder on which to cry. Judith Donath, Nicole Ellison, Scott Golder, Bernie Hogan, Eszter Hargittai, Amanda Lenhart, Liz Lawley, Alice Marwick, Clay Shirky, Fred Stutzman, and Michele Ybarra provided ongoing counsel about researching social network sites and helped me believe in what I was doing. Marc Davis, Henry Jenkins, Barrie Thorne, Nancy Van House, and Barry Wellman were always there to challenge me to think wider and deeper while providing ongoing mentorship and support.

During this process, I had the great fortune of calling many different communities home. The iSchool at Berkeley was an intellectually stimulating environment where I grew as a scholar. I was lucky to learn from brilliant faculty 
and be surrounded by a fantastic cohort of fellow $\mathrm{PhD}$ students. I am especially grateful to Joe Hall, Dan Perkel, Vivien Petras, Dilan Mahendran, Christo Sims, and Yuri Takhteyev for asking me tough questions and helping me work through the answers. My fellowships at the USC Annenberg Center and Harvard Berkman Center were key to exposing me to new ideas, and I am especially grateful to Jonathan Aronson, Dan Gillmor, John Palfrey, Doc Searls, Wendy Seltzer, Jimmy Wales, David Weinberger, Jonathan Zittrain, Ethan Zuckerman, and the Digital Natives team for their crazy ideas. Working at Yahoo!, Google, and Tribe.net helped me get an inside scoop on how technology is produced and disseminated and I am particularly indebted to Adam Bosworth, Ian Rogers, Marc Pincus, and Evan Williams for believing in me. I am also grateful to my agent Wes Neff for putting me in front of a wide range of audiences who forced me to think through my ideas.

I was lucky to do my fieldwork as part of a much larger project funded by the MacArthur Foundation concerning youth, learning, and new media. I am indebted to the MacArthur Foundation for funding this project and am especially thankful to John Seely Brown, Ben Stokes, and Connie Yowell for their commitment to my research. It was a blessing to embark on this project surrounded by a community of like-minded scholars working on similar studies. The 28-person Digital Youth team assembled by Mimi Ito, Peter Lyman, and Michael Carter provided the ideal intellectual space for working out the puzzles in my dissertation. I am especially grateful for long conversations and debates with Becky Herr-Stephenson, Heather Horst, and CJ Pascoe. 
I am beholden to Sam Jackson and Ann Murray, my research assistants, for tirelessly tracking down online content for me and to Karen Bleske, my copy editor, for making sure this document is legible. All of my mistakes in this document are my fault; I had the wise idea of doing "just one more" revision before submitting.

Throughout the country, various adults helped me connect with teenagers in their communities, including Carrie Benseler, Irma Carranza, Ben Chun, Doug Elmer, Anastasia Goodstein, Janet Hawk, Tom Hicks, Rachel Hunt, Zayira Jordan, Dina Kraemer, Hollie Mendenhall, Jeff Plinsky, Julie Tereshchuk, and Kristi Williams. I am deeply appreciative of their kindness. I am also indebted to those who helped me gain access to other forms of data, including Tom Anderson, Bich Ngoc Cao, Vicki Davis, Charlene Li, and Miriam Simun.

Finally, and most important, I want to acknowledge and thank the hundreds of teens who took the time to talk to me and, especially, those who approached me to help me get better clarity and those who took the time to meet me for an interview. Without their voices, this project would be meaningless. It is my hope that this document will reach all of their parents, because so many of them asked me to call their parents and help them understand that what they were doing was simply normal. 


\section{Chapter 1: Introduction}

I met Amy, a black-white 16-year-old in Seattle in early 2007. She told me that she loved MySpace. She logged in every chance she could. She took pride in crafting and regularly updating her profile, a colorful montage of photos and text that conveyed who and what mattered to her. She used various communication channels on MySpace to leave messages for her friends and socialize with her peers. Her MySpace profile displayed more than 5,000 comments, mostly textual snippets that reflected an ongoing conversation between her and her classmates. Amy and her boyfriend engaged in online public displays of affection through the use of photos, comments, blog posts, and text. MySpace also became a site of the drama that ensued after they broke up. While she simply erased his existence from her profile, prompting her friends to leave comments about what happened, he used his profile to disparage her. When I asked Amy why she spent so much time there, her answer was simple: "My mom doesn't let me out of the house very often, so that's pretty much all I do, is I sit on MySpace and talk to people and text and talk on the phone, cause my mom's always got some crazy reason to keep me in the house." Although Amy relishes the opportunity to have a social life while restricted to her house, her participation in MySpace has caused new tensions between Amy and her mother.

Amy's story is fairly typical. Between 2004 and 2007, many American teens

joined social network sites where they crafted digital self-representations and 
socialized with their peers, much to the chagrin of their parents. For many of them, gathering with friends was more viable on MySpace or Facebook than in person. Social network sites supported a wide array of everyday teen practices and much of what takes place in these environments parallels longstanding teen practices. At the same time, the mediated nature of these digital environments inflects everyday practices in new ways and widespread teen engagement with these sites has reshaped certain aspects of teen sociality.

\subsection{My Project}

To examine the interplay between American teenagers and this new technological form, I embarked on a 2.5-year study of teen participation in social network sites and other emergent forms of social media. Moving between online and offline environments, I interviewed and observed teens throughout the United States in order to understand how they adopted social network sites into their lives and how their lives were shaped by their engagement with these sites. Like scholars before me (Ito 2002; Thorne 1993), I approached my fieldwork with the belief that the practices of teenagers must be understood on their own terms, divorced from the desires or expectations of adults. I believe that teens' activities and logic can be understood as a rational response to the structural conditions in which they are embedded. Thus, I focused on understanding those structural conditions.

As my project unfolded, I focused on three sets of relations that play a dominant role in teen life: self and identity, peer sociality, and parents and adults. I examined 
how teens' sociotechnical practices supported and complicated all three sets of relations. I analyzed how teenagers used social media to reproduce traditionally unmediated practices, how teens altered their practices to accommodate technical features, how teens repurposed technology to meet their needs, and how teens managed complications that arose as a result of their sociotechnical participation. In doing so, I began to recognize a set of technical properties (persistence, searchability, replicability, and scalability) that destabilized core teen relations and a set of dynamics (invisible audiences, collapsed contexts, and the blurring of public and private) with which teens were forced to contend when participating in these environments. While these properties and dynamics certainly do not determine teen practices, they reshape the environment that teens inhabit and, thus, play a role in how teens negotiate identity, peer sociality, and interactions with adults.

I found that teen participation in social network sites is driven by their desire to socialize with peers. Their participation online is rarely divorced from offline peer culture; teens craft digital self-expressions for known audiences and they socialize almost exclusively with people they know. Their underlying behaviors remain relatively stable, but the networked, public nature of social network sites like MySpace and Facebook is particularly disruptive to more traditional forms of interaction. In crafting a profile, teens must manage a level of explicit selfpresentation before invisible audiences that is unheard of in unmediated social situations. The publicly articulated nature of marking social relations can prompt new struggles over status and result in heightened social drama, but as teens learn to 
manage these processes, they develop strategies for maintaining face in a social situation driven by different rules. The same structural forces that allow teens to interact with broad peer groups in a new type of public space also make their behaviors much more visible to those who hold power over them, prompting new struggles over agency and access to public life. Technical properties can often complicate teens' relations to peers and adults, but my findings illustrate that teenagers often develop powerful strategies to adapt to the logic of new genres of social media. As teens learn to navigate social media, individual practices and collective social norms evolve to account for the infrastructure.

To explore and theorize teens' sociotechnical practices, I integrate social science theories concerning everyday practices with a theory of technology that accounts for instabilities in the structural conditions of the environment in which those practices occur. The result is an ethnographic account of how social media has taken social situations out of traditional contexts and how American teens develop strategies for making sense of these environments in order to engage in everyday practices that are important to them.

\subsubsection{Dissertation Organization}

The core of this dissertation is organized around the three sets of relations central to teen life-identity, peer sociality, and power relations. Chapter 4 examines the intersection of social network site profiles and identity, Chapter 5 describes how social network sites shape teen sociality and peer relations, and Chapter 6 accounts 
for how adults' restrictions and fears configure adult-teen interactions in the context of teens' sociotechnical practices. In each of these chapters, I begin by laying out a relevant theoretical framework before turning to analyze how teens are managing each set of relations in conjunction with their participation in networked publics. Chapter 4 builds on Goffman's analyses of impression management, presentation of self, and behavior in social situations (Goffman 1959, 1963, 1966, 1967). Chapter 5 considers how Eckert's social categories (Eckert 1989) and Milner's status rituals (Milner 2004) fare in digitally mediated environments. Chapter 6 extends Valentine's analysis of how children lost access to physical public spaces (Valentine 2004). In each chapter, my aim is to use ethnographic fieldwork to describe teens' everyday lives in relation to the theoretical backdrop and technology to account for what has and has not changed.

While each analytic chapter in my dissertation addresses a different body of theoretical concerns, three core theoretical frameworks are woven throughout my dissertation: teenagers, technology and change, and networked publics. The remainder of this Introduction maps out these central theoretical frameworks.

To support my core ethnographic chapters, Chapter 2 outlines the ethnographic approach that I took in this project and Chapter 3 provides a historical overview of social network sites and teen engagement with social media. For those seeking more detail on my subjects or on the technology, Appendix 1 provides brief biographical information on all teens quoted in this dissertation and Appendix 2 details some of 
the core features in MySpace and Facebook. A third Appendix includes the Creative Commons license that modifies the copyright claims I make to this dissertation.

Finally, my concluding chapter addresses the implications of my findings and analysis with respect to conceptions of networked publics. Unlike other chapters in this dissertation, I step out of analytic mode in Chapter 7 to consider what my work means for broader theoretical and social conversations. The tone of this chapter is intentionally more reflective and less grounded and I offer my views on how I believe my work can be applied.

\subsection{The Social Construction of Teenagers}

My dissertation is fundamentally about teenagers and youth culture. Teenagers are not a native category but rather a social invention (Hine 1999). Although some psychologists and biologists frame childhood as a series of developmental stages (Erikson 1959; Piaget 1969), other social scientists argue that childhood is a social construct (Buckingham 2000; Corsaro 1997; James et al. 1988; Postman 1994) and that age distinctions more adequately mark status than any meaningful psychological stage (Chudacoff 1989). These scholars and other historians (Hine 1999; Savage 2007) argue that the social categories of childhood and teenager emerged for varying social, political, and economic reasons and were justified through developmental psychology. 
The social position of today's teenagers is constructed and configured by the combination of historical views of teens as both vulnerable and dangerous (Buckingham 2000; Cohen 1972) and the ways in which adults reinforce those beliefs through fear, age segregation, and control (Horne et al. 2005; Skelton and Valentine 1998; Valentine 2004). Teens' networked experiences are shaped by these factors, as teens are viewed to be both vulnerable and dangerous online and, as discussed in Chapter 6, adults frequently seek to control their online activities as well. By taking teenagers and their activities seriously, my analysis confronts the ways in which teenagers are socially constructed.

The constructs of childhood, adolescence, and teenager are historically and culturally dependent, even within Euro-American frameworks. Aries (1962: 411) notes that in European medieval times, "children were mixed with adults as soon as they were considered capable of doing without mothers or nannies, not long after tardy weaning (in other words, about the age of seven).” According to Postman (1994: 13-14), this is because seven is the age at which children have command over speech, the only skill necessary to participate in adult life. Postman argues that oral competence prompted the Catholic Church to deem children capable of reason and understanding right from wrong at the age of seven. With the technological invention of the printing press, adulthood was reconstructed and demarcated by reading competence (Postman 1994: 17-18). This new construction created a liminal stage between childhood and adulthood, a stage later called "adolescence" by the nineteenth-century psychologist G. Stanley Hall. While Hall (1908) 
distinguished between the social construct of "adolescence" and the biological period of puberty, his work is often used to affirm the notion that adolescence is a unique cognitive stage.

The early twentieth century brought about tremendous reform in American society with respect to youth. Moral reformers actively called on the government to curb perceived problems of youth deviance by introducing mandatory education that extended into the teen years. In constructing the idea of adolescence, Hall sought to support these reforms; he believed that delaying children's introduction into the world of work would be beneficial and education would help civilize the “savage” young people (Savage 2007: 66-67). In his efforts to turn adolescence into a carefree period not weighed down by responsibilities, Hall helped shift the societal view of a 14-year-old from an inexperienced and energetic adult to a vulnerable and irresponsible child. Hall's work fueled the intensification of norms centered on age in the early 1900s (Chudacoff 1989: 65-91), including sparking the juvenile court reform movement and the rise of the compulsory public schooling movement (Hine 1999: 172,198). The societal construction of adolescence provided fodder for the business class to project its values onto youth (Savage 2007: 93).

One result of compulsory high school was age segregation. With teens separated from adults for most of the day, their social interactions became peer-driven and a peer society emerged (Chudacoff 1989). Schools reinforced peer-based social structures through the introduction of school-run activities and sports. Age 
segregation gave rise to the introduction of the term "teenager" as a label for a unique marketable youth demographic (Hine 1999). Teenagers became consumers and while the freedom to consume appealed to teens, it became a double-edged sword because consumer-driven youth culture "depends on young people's isolation from the adult world" (Hine 1999: 226). Through the creation and reinforcement of youth culture, consumerism reified divisions between adults and teens. As teens struggled to locate themselves in society, some youth insisted that adults recognized them and treated them as equals. In 1945, the New York Times Magazine published "A Teen-Age Bill of Rights," which included the rights to: "let childhood be forgotten," "to make mistakes, to find out for oneself," "to have rules explained, not imposed," and "to be at the romantic age" (Savage 2007: 455).

Sociologists of youth culture often identify the 1950 s as a pivotal period that saw the emergence of many of the dynamics that define contemporary youth peer culture and adult attitudes toward youth (Chudacoff 1989; Frank 1997; Gilbert 1986). The factors at play were already in motion-compulsory high school, youthoriented commercial culture, and the age-segregated peer culture that dominated youth's everyday negotiations over status and identity. Yet it was the 1950s when these factors solidified and youth became a demographic in most sectors of society. Adults further fragmented youth in the mid-1960s when, whether because of actual resistance or because of fears of potential mass rebellion, adult society began to worry about the corruption of teens by older peers. While adult society disliked the 1960s youth culture epitomized by those resisting the Vietnam War, it abhorred 
efforts by teens to politically align themselves with older peers and reject the rules and social controls of schools (Hine 1999: 269-270). Issues like drugs, sex, spirituality, and politics drove a wedge between the generations. While generational tensions had occurred before, hysteria over the highly publicized behaviors of some older youth made parents worry about their teen children. Adults began cracking down on the freedoms that teens had, trying to separate them from the rest of youth culture. With the draft, the lowered voting age, and the laws targeted at "minors," 18 became a constructed and realized age marker that separated teens from adults. These systems that configure teens typically have more to do with control and power than biological development.

Age continues to play a salient role in the lives of teens and age consciousness extends online. As such, issues of age and the way in which teenagers are constructed are woven throughout this dissertation. In Chapter 4, I discuss how articulating one's age is a universal practice in social network sites and teens weigh many factors when deciding what age to list. Age segregation also extends online, as teens' social worlds consist almost exclusively of peers their age. Thus, the inspection of peer sociality in Chapter 5 centers on same-aged peers. Furthermore, the adult fears and efforts to maintain control that have made age so salient extend online. Chapter 6's examination of the complicated power dynamics between teens and adults is rooted in the way in which teens are now configured. My efforts to recognize teens as capable people responding to their environment are threaded throughout. 


\subsection{Technology and Change}

While my focus is primarily on teens' practices, technology plays a central role in my analysis, and theories of technology and change are woven deeply into my analysis and arguments. In particular, I take a social constructivist approach with respect to technology, teens' engagement with networked publics, and the change that occurs in relation to technology. This approach explicitly counters the notion that technology determines practice and social outcomes. I believe that such "technological determinism" is a reductionist philosophy that fails to account for the complex ways in which technology and society interact.

The appeal and problems of technological determinism can be viewed in light of "hacking" culture, which simultaneously reflects the ways in which systems designers expect certain outcomes and how hackers reconfigure technology to subvert those outcomes (Jordon 2008). While both designers and users shape a technology, their relationship with one another can be fraught, especially when designers believe that their design should prompt specific responses by users and users see otherwise, as in the case of hackers. In some instances, technologists try to "configure the user" (Grint and Woolgar 1997) by commanding users to behave in a certain way and punishing them when they do not comply. This can rupture the relationship between users and the technology, often resulting in even more undesirable responses. Such was the case with Friendster, an early social network site that was designed to be an online dating service. When users rejected that 
assumption and chose to use it for varied creative activities, the company began eliminating features that the users leveraged and deleting the accounts of those who did not share the company's intentions; this prompted many users to depart, including those who ended up constituting MySpace's early adopters (boyd 2006a).

Arguments that vilify technology_like books that blame social media for giving everyday people too much power (Keen 2007) or for stupefying youth (Bauerlein 2008)-typically stem from technological determinist views that are fed by fears of what could be rather than what is. Rejecting technological determinism requires getting away from the notion that technology is inherently positive or negative. At the same time, it is not without value. This situation is best described by Kranzberg's First Law: "Technology is neither good nor bad; nor is it neutral" (Kranzberg 1986: 454-548). A technology's value is shaped by its social construction-how designers create it and how people use it, interpret it, and reconfigure it. It is not an outcome of the technology alone or its potential.

To make social constructivism applicable to analyzing technological systems and artifacts, Pinch and Bijker (1984) introduced a theoretical and methodological framework that was labeled SCOT, or "social construction of technology" (Bijker et al. 1987). SCOT explicitly accepts that technology shapes and is shaped by those who use it and the society in which it is embedded, and those behind SCOT offered a set of interventions and models for analyzing technological systems and artifacts. 
The model presented by Pinch and Bijker (1984) consists of five key elements that researchers should account for in their data collection and analysis:

- Relevant Social Groups: all who are connected to, affected by, and relevant to the technological artifact or system, including both users and producers.

- Problems and Conflicts: struggles between different relevant social groups in relation to the technology and issues that arise because of different usage patterns and practices.

- Interpretive Flexibility: people engage with technologies in different ways and technologies must not be presumed to have one meaning, purpose, or interpretation.

- Design Flexibility: there are countless ways of designing a particular artifact and design should not be presumed to follow a linear or logical path.

- Closure and Stabilization: interpretations and the design of a technology may stabilize, offering analytical and rhetorical closure, but new relevant social groups or interpretations may also destabilize them.

Upon introduction, the SCOT model was immediately challenged. Agreeing with their premise, Russell (1986) argued that the relativist approach taken by Pinch and Bijker (1984) moves toward the idea that technology is neutral and that their process is internally conflicting and fails to account for social structure, power, and 
access. He also calls into question the appropriateness of drawing on work from the sociology of science. Pinch and Bijker (1986) accept Russell's criticisms and welcome his comments on how SCOT can incorporate a theory of social structure, but they also push back against his rejection of the value of science studies and think it is unfair for Russell to have expected them to account for a wide swath of literature and analytic frameworks in a short article. The rest of the article expounds on their approach in an effort to counter Russell's criticisms. The SCOT framework is further detailed in Bijker, Pinch, and Hughes (1987), alongside articles by scholars who build on this model.

Not focusing on a history of technology, I did not formally adopt the SCOT methodology, but SCOT shaped my thinking. I was conscious to account for different relevant social groups, including diverse groups of teens, the producers of the technologies they use, and the adults who shape teens' lives. Interpretive flexibility runs throughout my fieldwork and analysis as I account for the different ways in which teens see social network sites, their features, and their relationship to the sites. Likewise, in accounting for the differences between MySpace and Facebook, I am directly drawing from SCOT's approach to design flexibility.

SCOT's footprint is also visible when I approach issues of change. While SCOT includes a discussion of stability and closure, my analysis is centered on an active sociotechnical phenomenon and a set of practices that are shaping and being shaped by technology. As a result, I am more attentive to the changes that are under way 
than to stabilizing forces. A social constructivist approach recognizes that the relationship between technology and practice is often in flux. Interpretive flexibility involves accounting for how interpretations change through time and how engagement with technology reshapes other practices. Likewise, in my analysis, I focus on the shifts in individual teens, between teens, and in relation to historical teens.

While I do not address SCOT explicitly in my analytic chapters, the approach to technology and change that is integrated throughout this dissertation focuses on the ways in which technological artifacts like networked publics shape and are shaped by teens and their practices. This is an ongoing process and my work is a snapshot in time. In that snapshot, I account for what has shifted, what has not, and what those shifts mean.

\subsection{Locating Networked Publics}

The third major theoretical framework that shapes my analysis concerns publics, and, in particular, networked publics. Networked publics are publics that are restructured by networked technologies. As such, they are simultaneously (1) the space constructed through networked technologies and (2) the imagined community that emerges as a result of the intersection of people, technology, and practice. Social network sites like MySpace and Facebook are networked publics, just like parks and other outdoor spaces can be understood as publics. Collections of 
people connected through networked technologies like "the blogosphere" are publics, just like those connected by geography or identity are.

Mizuko Ito introduces the notion of networked publics to "reference a linked set of social, cultural, and technological developments that have accompanied the growing engagement with digitally networked media" (Ito 2008: 2). I agree with her framing, but I extend the term further to account for the resultant spaces and collectives that emerge because of these developments.

\subsubsection{Public and Publics}

The concept of networked publics is slippery because the concept of "publics" is messy. The term "public" is contested, has multiple meanings, and is used across disciplines to signal different concepts. During my interviews, I found that teens also struggle to define this term and rely on multiple meanings to approach a definition from different angles. When used descriptively, "public" is often in opposition to the equally slippery concept "private" to signal potential access. For example, Lila, a Vietnamese 18-year-old from Michigan, told me that "when it's public, anyone can see it." As a noun, "public" typically refers to certain collections of people. Taking another stab at marking what is public, Lila notes that "you think about public as like within your big group of friends." In this way, she bounds public through the construct of a population. In addition to such social configurations, there are also civic and cultural takes on how those collections are constructed. 
Although nuanced, different approaches to public often refer to deeply intertwined notions.

Hannah Arendt argues that one approach to thinking about public is that which "can be seen and heard by everybody and has the widest possible publicity" (Arendt 1998: 50). Arendt's view is that bringing things into public affords them a reality otherwise inaccessible. While this can certainly have costs, Arendt notes that "the presence of others who see what we see and hear what we hear assures us of the reality of the world and ourselves" (Arendt 1998: 50). Her approach to "public" focuses on potential accessibility of spaces and information to wide audiences, including the possibility of strangers. While Arendt's approach is primarily concerned with civic or political publics, this framing can also be understood in cultural and social terms.

Another approach is to construct "public" as a collection of people who share "a common understanding of the world, a shared identity, a claim to inclusiveness, a consensus regarding the collective interest” (Livingstone 2005: 9). In this sense, public may refer to a local collection of people (e.g., one's peers) or a much broader collection of people (e.g., members of a nation-state). Livingstone’s approach focuses on how media helps shape collectives by providing a common context, but this definition can also be seen in a civic frame. In marking a collection of people organized by nationality as an "imagined community," Benedict Anderson (2006a) 
gets at the way that public is not a definable set of people but a flexible category where people may conceptualize but do not control the boundaries.

Traditionally, public is often marked by the definitive article "the," implying that there is only one public. Yet when the United States President addresses "the public," he is not talking to the same collection of people that the Zimbabwean President is addressing when he speaks. Presidents from different countries are speaking to different constituents and, thus, assume different collective norms and values. Rarely does a politician speaking about "the public" mean all who are living regardless of nationality, residence, or language. Using the indefinite article allows us to recognize that there are different collections of people depending on the situation and context. This leaves room for multiple "publics." Individuals often engage with and are members of different publics and they move between them fluidly. Publics are not always distinct from one another and there are often smaller publics inside broader publics. For example, Lila's cohort may represent a public, but they exist within other publics, including those labeled as teens, Americans, and consumers. Just as publics are made of smaller publics, there are also collectives that emerge to challenge the normative cultural ideas of the public to which they implicitly belong; these can be understood as "counterpublics" (Warner 2002).

Publics are most commonly theorized when one thinks about the political role that they play. Much of this is rooted in, conversational with, or challenging of Habermas's historical analysis of a public sphere as a category of bourgeois society 
(Calhoun 1992a; Crossley and Roberts 2004; Habermas 1991; Ryan 1992).

Habermas is particularly dismissive of depoliticized publics that involve a “preoccupation with consumption of culture" (Habermas 1991: 177), although he reserves most of his venom for modern broadcast media's role in creating "a public sphere in appearance only" by allowing people to examine and consume tastes (Habermas 1991: 171). Craig Calhoun argues that one of Habermas's weaknesses is his naive view that "identities and interests [are] settled within the private world and then brought fully formed into the public sphere" (Calhoun 1992b: 35). Feminist scholar Nancy Fraser argues that publics are not only a site of discourse and opinion but "arenas for the formation and enactment of social identities" (Fraser 1992). Even in the realm of civics, there is tremendous overlap between the political and cultural nature of publics.

Broadcast media restructured publics by changing the ways in which information flowed and by supporting the formation of collectives organized around a shared understanding of the world. Networked media-like the Internet-is another restructuring force. Habermas was wary of broadcast media because of the ways in which it altered political life; he relishes the introduction of the printing press for enabling an informed public, but he blames contemporary broadcast media for creating a consumer public that he sees as uncritical and irrational (Habermas 1991). There is little doubt that each new wave of media has fundamentally altered the structure of many aspects of everyday life (McLuhan 1964), but it is not clear that new media is the destroyer of culture that Habermas portrays it to be. As a reshaping 
agent, media introduces changes that can be seen as both positive and negative depending on one's perspective and situation. Habermas's nostalgic idea of a public sphere is unquestionably bourgeois while the publics created and made visible through mass media are more egalitarian. What Habermas sees as a decline in informed citizenry due to media may simply be the broadening of the public to include groups who were previously disempowered.

While political theory has dominated discursive efforts to define "public," cultural and media studies offers a different perspective. In locating the term "public," Sonia Livingstone (2005) notes that publics are sometimes synonymous with "audience" as both refer to a group bounded by a shared text, whether a worldview or a performance. The audience produced by media is often by its very nature a public. Additionally, as civic publics are highly mediated, politicians are synonymously speaking to a public that is an audience. When one thinks of publics in relation to audiences, questions of agency and participation emerge. Yet as Henry Jenkins $(1992,2006)$ has argued, audiences are not necessarily passive consumers. This builds on de Certeau's (2002) argument that consumption and production of cultural objects are often intimately connected. Mizuko Ito extends this to say that "publics can be reactors, (re)makers and (re)distributors, engaging in shared culture and knowledge through discourse and social exchange as well as through acts of media reception" (Ito 2008: 3). Both social scientists and theorists also address publics implicitly in their efforts to locate what constitutes society or culture or in 
order to map out everyday practices that take place in public spaces (e.g., Bourdieu 1984; Goffman 1966; Simmel 1972).

Because the concepts addressed by "public" are interconnected, I intentionally leave them messy and pull from different strands. In the context of my dissertation, a public is both a space where people may gather, interact, and be viewed and also an imagined community of people who share similar practices, identities, and cultural understandings. That which is public is potentially but not necessarily visible.

Furthermore, the boundaries of publics may be generally imagined but not necessarily understood. Media influences, configures, and is configured by publics. Publics play a role in all aspects of social life, including political and cultural dimensions.

\subsubsection{Teenagers and Publics}

Neither political nor cultural formations of publics properly account for teenagers. While scholars of feminism and queer theory examine the ways in which marginalized populations are excluded from and create their own publics (Warner 2002) or struggle to gain access to hegemonic publics (Fraser 1992; Ryan 1992), age-based marginalization operates differently. Teenagers are systematically barred from both political and cultural publics, but their exclusion is also temporary; once they are "of age," they attain a plethora of rights and access to places to which they were previously denied. As discussed earlier, their collective demands for access have often been met with resistance and increased restrictions. Their ability to organize 
their own publics or gain access to adult publics is not only limited by their status and mobility but also by the ways in which they leave their pleas behind once they have transitioned into adulthood. Also, unlike women, people of color, and queer individuals who have increasingly gained freedoms and rights with respect to publics through time, teenagers' freedoms and rights continue to erode and teenagers are more marginalized today than they were a century ago (Hine 1999).

While teens' disenfranchisement is temporally short-lived, it fractures public life at a deeper level. Teens are trained to fear publics (Valentine 2004), complicating later participation. The exclusion of teens from adult publics limits opportunities for intergenerational dialogue, furthering age segregation and decreasing social solidarity. Exclusion also keeps teens disconnected from incentives to contribute to the public good in the form of civic or political action. Teens are also relatively powerless to voice when they are being harmed, especially when that harm is taking place in the private spaces to which society restricts them. As women gained access to male-dominated publics and feminism unfolded, the private violence that women experienced became a public concern (Fineman 1994). Access to publics is key to combating the kinds of harm that fracture individuals and society as a whole.

It is not that teenagers lack the desire to engage in publics but, as detailed in Chapter 6, they lack the means to participate in broad cross-generational publics and the hegemonic on es controlled by adults. Teens seek access to physical spaces where publics manifest, but increasingly, their access is curtailed (Valentine 2004). 
Although teens' access to these types of publics is limited, teens are not totally disconnected from publics. The peer worlds that teens create at school can be understood as publics and youth subcultures can be interpreted as counterpublics (Warner 2002). Many teens have some access to controlled public spaces, most notably commercial publics. Yet the surveillance and restrictions in these spaces do not necessarily afford the opportunities of publics. Teens' early adoption of networked publics also highlights their desire to engage in publics, particularly for social and cultural purposes.

\subsubsection{Publics, Networked}

Networked media amplifies broadcast media's advantages and disadvantages, enabling everyday people to be both consumers and producers of cultural content in new ways. This is both potentially beneficial and problematic. People have more opportunities to make their voices heard, but the ruptures brought on by broadcast media-such as those to publicity, privacy, and social context-also extend to a much wider group. What distinguishes networked publics from unmediated or broadcast publics is the underlying structure. New forms of media-broadcast or networked-reorganize how information flows and how people interact with information and each other.

Both broadcast mechanisms and networked technologies reconfigure the architecture of publics. As a frame, architecture sheds light on how publics are structured. While a definition of architecture is contested (Hays 2000; Shepheard 
1994), in the everyday sense, architecture typically evokes the image of the design of physical structures-buildings, roads, gardens, and even interstitial spaces. The product of architecture can be seen as part engineering, part art, and part social configuration, as structures are often designed to be variably functional, aesthetically pleasing, and influential in shaping how people interact with one another. Frank Lloyd Wright takes a more inclusive view by arguing that "architecture is life" (Wright and Gutheim 1941: 257).

In technical circles, a system's architecture is the organization of code that produces digital environments. Code is the cornerstone of digital architecture. Drawing on William Mitchell (1995: 111), Lawrence Lessig (2006: 1-8) argues that "code is law" because code regulates the structures that emerge. James Grimmelmann argues that Lessig's use of this phrase is "shorthand for the subtler idea that code does the work of law, but does it in an architectural way" (Grimmelmann 2004: 1721). In looking at how code configures digital environments, both Mitchell and Lessig analyze the ways in which digital architectures are structural forces.

Physical structures are a collection of atoms while digital structures are built out of bits. The underlying properties of bits and atoms fundamentally distinguish these two types of environments, define what types of interactions are possible, and shape how people engage in these spaces. More than a decade ago, Nicholas Negroponte (1995) mapped out some core differences between bits and atoms to argue that 
digitization would fundamentally alter the landscape of information and media. He pointed out that bits could be easily duplicated, compressed, and transmitted through wires; media that is built out of bits could be more easily and more quickly disseminated than that which comprises atoms. This would mean that media built on bits would be cheaper to distribute than media built on atoms. He forecast the transformation of enterprises organized around the distribution of information and media, including the implications for news and music industries. During that same period, Mitchell (1995) argued that bits do not simply change the flow of information, but they alter the very architecture of everyday life. Through networked technology, people are no longer shaped just by their dwellings but by their networks (Mitchell 1995: 49). The city of bits that Mitchell lays out is not configured just by the properties of bits but by the connections between them.

As both a space and a network of people, networked publics are fundamentally shaped by the properties of bits, the connections between bits, and the way that bits and networks link people in new ways. Networked publics are not just publics networked together, but they are publics that have been transformed by networked media, its properties, and its potential. As a form of architecture, networked publics are regulated by the properties of bits. These properties configure the structure of networked publics, introduce new possible practices, and shape the interactions that take place. 


\subsection{The Structure of Networked Publics}

Four properties that exist because of bits play a significant role in configuring networked publics: persistence, replicability, scalability, and searchability. These properties are intertwined and codependent, and they help produce three dynamics that shape people's experience with networked publics: invisible audiences, collapsed contexts, and the blurring of public and private. To account for the structure of networked publics, I want to map out these different elements, situate them in a broader discussion of media, and suggest how they shape networked publics.

Media reshapes publics by introducing new properties into environments. Using McLuhan's phrase, media is an "extension of man" (McLuhan 1964). Media amplifies, records, and spreads information and social acts. This affects how people negotiate publics because information and social acts are essential to publics. As people adjust to these properties, new practices emerge. While these properties and dynamics do not determine social practice, they do destabilize core assumptions people make when engaging in social life and, thus, play a significant reshaping role when one thinks about publics. When left unchecked, media can play too powerful a role in controlling information and configuring interactions. This is one fault line that prompts resistance to and demonization of new forms of media. It is also why media literacy is essential—understanding the role that these properties play in shaping the environment is important to public participation. 


\subsubsection{Properties of Networked Publics}

The content of networked publics is inherently made out of bits. Both selfexpressions and interactions between people produce bit-based content in networked publics. As Negroponte (1995) explains, the features of bits are quite different from those of atoms. Because of these features, bits are easier to store, distribute, and search than atoms. These features of bits configure the four properties that are key to networked publics:

- Persistence: online expressions are automatically recorded and archived.

- Replicability: content made out of bits can be duplicated.

- Scalability: the potential visibility of content in networked publics is great.

- Searchability: content in networked publics can be accessed through search.

These four properties structure network publics and the interactions that take place in them. Many of these properties are not unique to networked publics-oral histories made stories persistent, the printing press replicated content with ease, broadcast media scaled the visibility of live acts, and librarians have long invested in approaches to searching for information. Yet in networked publics, these properties are a part of the environment by default and are interconnected in new ways. Furthermore, because they play a role in all mediated interactions and because networked publics play a significant role in the lives of many teens, teens who participate in social network sites must account for these properties during everyday acts and interactions. 
Technology and new forms of media have reshaped publics at multiple points in history. For example, writing allowed people to document events for the record. At one level, this can be viewed as an affront to oral histories and the role of storytellers. Some might say that writing is more permanent, more reliable, and easier to circulate. Others might argue that physical records can be destroyed more easily than human memory, or that written records can be easier to misinterpret when taken out of context while storytellers are more likely to adjust the narrative for the given situation so that it is best understood. Yet few would argue that the shift from orality to literacy changed the nature of public information (Ong 2002). While new technologies have their strengths and weaknesses, it is how they are adopted that changes cultural practices.

Just as writing changed how text could be recorded, photography changed the way that moments could be visually captured. Photography was less laborious than painting, but Walter Benjamin (1969) rightfully argued that it changed the essence of the art of capturing a moment in time. The printing press allowed for easy reproduction of news and information, increasing the potential circulation of those aware of events (Eisenstein 1980). The resulting newspapers and the rise of journalism were considered key to the idea of an "informed public" and, thus, democracy (Starr 2005). Broadcast media like TV and radio took this further, making it possible for events to be simultaneously experienced across great distances, radically scaling the potential visibility of a given act and reshaping the public sphere (Starr 2005). 
Through time, the technologies behind these information revolutions became broadly accessible. Cameras and video recorders are now omnipresent, allowing people to record their experiences. Printers are now household items, allowing people to produce their own documents. As these innovations became everyday gadgets, the channels and networks for scalable distribution were still limited. For example, the government regulates spectrum, making it difficult for everyday citizens to broadcast their own radio or TV stations, but this did not stop "pirates" from creating their own broadcast publics (Walker 2004). In the same way, "zines" emerged as alternatives to traditional news and magazine publishing, leveraging both social networks and the post office for distribution (Duncombe 2008). The Internet introduced new possibilities for distribution; blogging alone allowed for the rise of grassroots journalism (Gillmor 2004) and a channel for anyone to espouse opinions (Rettberg 2008). The Internet made it possible for anyone to broadcast content and create publics, although it did not guarantee an audience.

The changes brought on by networked media are more pervasive than those by earlier media. While recording devices allow people to record specific acts in publics, unmediated publics are not inherently recording whatever takes place there. The structural configurations of networked publics do, by default, record everything in the process of converting digital expressions to bits before they are transmitted across the network. Likewise, the process of dissemination results in the reproduction of every bit sent across the network. While original records and duplicated records could be deleted (or, technically, overwritten) at any point in the process, the 
"persistent-by-default, ephemeral-when-necessary" dynamic is new to networked publics. Furthermore, since deletion runs counter to the cultural ethos and reproduction of content is rampant, tracking down and deleting content once it is contributed to networked publics is often futile.

Because that which is contributed to networked publics is persistent and replicable (and networked) by default, it is possible for acts to be viewed more broadly. Physical spaces are limited by space and time, but, online, people can connect to one another across great distances and engage with asynchronously produced content over extended periods. This allows people to work around physical barriers to interaction and reduces the cost of interacting with people in far-off places. At the same time, persistence and replicability complicate notions of "authenticity," as acts and information are not located in a particular space or time and, because of the nature of bits, it is easy to alter content, making it more challenging to assess its origins and legitimacy. This issue has long been a part of discussions about reproductions and recordings, with Benjamin (1969: 220) suggesting that art detached from its time and space loses its "aura" and Auslander (1999: 85) arguing that aura is in the relationship between performances and their recordings.

Authenticity is at stake in networked publics because altering content in networked publics is both easy and common. Code, text, images, and videos are frequently modified or remixed. While remix is politically contentious, it reflects an 
active and creative engagement with cultural artifacts (Lessig 2005, 2008), amplifying ongoing efforts by people to make mass culture personally relevant by obliterating the distinctions between consumers and producers (de Certeau 2002). How people alter content in networked publics varies. Alterations can be functional (e.g., altering code to make it work in a new environment), aesthetic (e.g., altering images to remove red eye), political (e.g., modifying famous photos to make political statements [Jenkins 2006]), or deceptive (e.g., altering text to make it appear as though something was said that was not). This magnifies questions of what is original, what is a copy, and when does it matter?

While there are limits to how many people can be in one physical space at a time, networked publics support the gathering of much larger groups, synchronously and asynchronously. Networked publics make one-to-many and many-to-many interactions far easier. In essence, networked media allows anyone to be a media outlet (Gillmor 2004) and with this comes the potential of scalability. Yet an increase in people's ability to contribute to publics does not necessarily result in an increase in their ability to achieve an audience. While a niche group may achieve visibility that resembles "micro-celebrity" (Senft 2008), only a small fraction receive mass attention while there is a "long tail" of participants who receive very small, localized attention (Anderson 2006b). In other words, scalability in networked publics is about the possibility of tremendous visibility, not the guarantee of it. Andy Warhol argued that mass media would guarantee that "in the future everyone will be world-famous for fifteen minutes" (Hirsch et al. 2002). As new media emerged, 
others contended that "in the future everyone will be famous for fifteen people" (Momus 1992; Weinberger 2002: 104). In networked publics, one commodity is attention. The rise in content producers creates an attention economy in which people must compete for visibility.

The potentials of scalability raise questions about the possible democratizing role that networked media can play when anyone can participate and contribute to the public good (Benkler 2006; Shirky 2008; Tapscott and Williams 2006). Unfortunately, networked publics appear to reproduce many of the biases that exist in other publics-social inequalities, including social stratification around race, gender, sexuality, and age, are reproduced online (Chen and Wellman 2005). Political divisions are reproduced (Adamic and Glance 2005) such that even when content scales in visibility, it may not cross sociopolitical divisions. Those using networked media to contribute to the dissemination of news selectively amplify stories introduced by traditional media outlets, replicating offline cultural foci (Zuckerman 2008). Although networked publics support mass dissemination, the dynamics of "media contagion" (Marlow 2005) show that what spreads depends on the social structure underlying the networked publics. In other words, scalability is dependent on more than just the properties of bits.

Habermas's frustration with broadcast media was rooted in the ways that broadcast media was, in his mind, scaling the wrong kinds of content (Habermas 1991). The same argument can be made concerning networked media, as what 
scales in networked publics is often the funny, the crude, the embarrassing, the mean, and the bizarre, "ranging from the quirky and offbeat, to potty humor, to the bizarrely funny, to parodies, through to the acerbically ironic" (Knobel and Lankshear 2007). Those seeking broad attention, like politicians and wannabe celebrities, may have the ability to share their thoughts in networked publics, but they may not achieve the scale they wish. The property of scalability does not necessarily scale what individuals want to have scaled or what they think should be scaled, but what the collective chooses to amplify.

Because of persistence, replicability, and scalability, a great deal of information is out there, waiting to be structured, organized, and accessed by those looking for it. This prompted a rise in the search industry as companies sought to find more efficient and effective ways for people to find what they were looking for. While librarians have long developed techniques to organize and access information and search has long been a core feature of computational systems, the developments of search post-Internet have been tremendous (Battelle 2005). On the Internet, people and their information are increasingly accessible through the powers of search in ways that have no offline parallels. For example, while my mother may have wished to scream "Find!" into the ether in an effort to determine where I was when I was out with friends, she could not. Today, online, a few keystrokes make it easy to identify someone's website or other digital representation. 


\subsubsection{New Dynamics Resulting from Networked Publics}

Analyzing how broadcast media transformed culture, Joshua Meyrowitz (1985) acutely recognized that the properties of media change social environments and, thus, influence people and their behavior. He examined how broadcast media's ability to rework scale reconfigured publics, altered the roles that people play in society, complicated the boundaries between public and private, collapsed distinct social contexts, and ruptured the salience of physical place in circumscribing publics. Just as many of the properties of networked media extend those of broadcast media, many of the dynamics that play out in networked publics are an amplification of those Meyrowitz astutely recognized resulting from broadcast media:

- Invisible audiences: not all audiences are visible when a person is contributing online, nor are they necessarily co-present.

- Collapsed contexts: the lack of spatial, social, and temporal boundaries makes it difficult to maintain distinct social contexts.

- The blurring of public and private: without control over context, public and private become meaningless binaries, are scaled in new ways, and are difficult to maintain as distinct.

In unmediated spaces, it is common to have a sense for who is present and can witness a particular performance. Through persistence, replicability, scalability, and searchability, networked publics introduce the possibility of audiences that are, for all intents and purposes, invisible. It may not be possible to see who is actually present 
at that moment, because either they are lurking and not showing themselves or because the technology does not make their presence visible. Furthermore, because audiences often perceive performances asynchronously, the audience may not be present at the time of the performance. When performing in networked publics, people are forced to contend with invisible audiences and engage in acts of impression management even when they have no idea how their performances are being perceived.

This dynamic has long been a part of certain professions. In producing content for the camera, microphone, or printing press, journalists and actors sometimes prepare for invisible audiences by imagining the audience and presenting themselves to that imagined audience. When TV began, studio audiences were tremendously common because it helped people gauge their performances. This audience was not the complete audience, but the feedback was still valuable for the performers. Likewise, some journalists perform for those who provide explicit feedback, intentionally avoiding thinking about those who are there but invisible. Performing for imagined or partial audiences can help people handle the invisible nature of their audience. These practices became a part of life in networked publics, as those who contributed tried to find a way to locate their acts. In an earlier study on blogging, a popular blogger I interviewed named Carl explained this process:

“There's actually enough [readers of my blog] at this point, that there's no way I could have a real sense of who all of them are. What I have is what I think a lot of 
fiction writers have, which is a readership that I write for that is not the whole readership, and not even representative of the readership, but it's what I aim for. And some of them are actual people. ... I know my mom reads my blog. I know SJ and JL read my blog. ... I know my exes read my blog. I know my coworkers read my blog. ... And so when I blog, I generally have one of those people in mind when I post.”

The techniques that Carl uses help him determine what is socially appropriate, interesting, or relevant. Knowing one's audience is a crucial step in understanding the social context. Without this information, it is often difficult to determine how to behave, let alone to make adjustments based on assessing reactions. The properties of networked publics lead to a dynamic in which people are forced to contend with a loss of context.

To complicate matters more, networked publics collapse contexts. This is an issue that Meyrowitz (1985) details in relation to how public figures were forced to negotiate broadcast media. Meyrowitz (1985: 43) recounts the experiences Stokely Carmichael, a civil rights leader, faced when he began addressing broad publics via television and radio in the 1960s. Until then, Carmichael had used very different rhetorical styles when speaking to blacks and whites, but when faced with electronic media, he had to make a choice. There was no neutral speaking style and Carmichael's decision to use black speaking style alienated white society. While Carmichael was able to maintain distinct styles as long as he was able to segment 
social groups, he ran into trouble when electronic media collapsed those social groups and with them, the distinct contexts in which they were embedded.

Networked publics force everyday people to contend with environments in which contexts are regularly colliding. Even when the immediate audience might be understood, the potential audience can be far greater and from different contexts. Maintaining distinct contexts online is particularly tricky because of the persistent, replicable, and searchable nature of networked acts. People do try to segment contexts by discouraging unwanted audiences from participating or trying to limit information to make searching more difficult or by using technologies that create partial walls through privacy settings. Yet a motivated individual can often circumvent any of these approaches.

Some people argue that distinct contexts are unnecessary and only encourage people to be deceptive. This is the crux of the belief that only those with something to hide need privacy. What is lost in this approach is the ways in which context helps people properly locate their performances. Bilingual speakers choose different languages depending on context, and speakers explain concepts or describe events differently when talking to different audiences based on their assessment of the audience's knowledge. An alternative way to mark context is as that which provides the audience with a better understanding of the performer's biases and assumptions. Few people detail their life histories before telling a story, but that history is often helpful in assessing the significance of the story. While starting every statement 
with "as a person with $\mathrm{X}$ identity and $\mathrm{Y}$ beliefs and $\mathrm{Z}$ history" can provide context, most people do not speak this way, let alone account for all of the relevant background for any stranger to understand any utterance.

Networked publics both complicate traditional mechanisms for assessing and asserting context as well as collapse contexts that are traditionally segmented. This is particularly problematic because, with the audience invisible and the material persistent, it is often difficult to get a sense for what the context is or should be. Collapsing of contexts did take place before the rise of media but often in more controlled settings. For example, events like weddings, in which context collisions are common, are frequently scripted to make everyone comfortable. Unexpected collisions, like running into one's boss while out with friends, can create awkwardness, but since both parties are typically aware of the collision, it can often be easy to make quick adjustments to one's behavior to address the awkward situation. In networked publics, contexts often collide such that the performer is unaware of audiences from different contexts, magnifying the awkwardness and making adjustments impossible.

Additionally, as networked publics enable social interactions at all levels, the effects of these dynamics are felt at much broader levels than those felt by broadcast media and the introduction of other forms of media to publics. These dynamics alter interactions among very large and broad collections of people, but they also complicate the dynamics among friend groups and collections of peers. They alter 
practices that are meant for broad visibility and they complicate-and often make public-interactions that were never intended to be truly public. This stems from the ways in which networked media, like broadcast media (Meyrowitz 1985), blurs public and private in complicated ways. For those in the spotlight, broadcast media often appeared to destroy privacy. This is most visible through the way tabloid media complicated the private lives of celebrities, feeding on people's desire to get backstage access (Turner 2004). As networked publics brought the dynamics of broadcast media to everyday people, similar dynamics emerged (Solove 2007).

Some argue that privacy is now dead (Garfinkel 2001) and that we should cope and embrace a more transparent society (Brin 1999). That is a naive stance, both because privacy has been reshaped during other transformative moments in history (Jagodzinski 1999) and because people have historically developed strategies for maintaining aspects of privacy even when institutions and governments seek to eliminate it (McDougall and Hansson 2002; Toch 1992). For these reasons, I argue that privacy is simply in a state of transition as people try to make sense of how to negotiate the structural transformations resulting from networked media.

People value privacy for diverse reasons, including the ability to have control over information about themselves and their own visibility (Rossler 2004: 6-8). Social network sites disrupt the social dynamics of privacy (Grimmelmann 2008). Most importantly, they challenge people's sense of control. Yet, just because teens 
are adopting tools that radically reshape their relationship to privacy does not mean they are interested in giving up their privacy.

Defining and controlling boundaries around public and private can be quite difficult in a networked society, particularly when someone is motivated to publicize something that is seemingly private or when technology complicates people's ability to control access and visibility. What remains an open question is how people can regain a sense of control in a networked society. Nissenbaum (2004) argues that we need to approach privacy through the lens of contextual integrity, at least in terms of legal protections. I believe that we need to examine teens' strategies for negotiating control in the face of structural conditions that complicate privacy and rethink our binary conceptions of public and private. While public and private are certainly in flux, it is unlikely that privacy will simply be disregarded.

The three dynamics discussed-invisible audiences, collapsed contexts, and the blurring of public and private-are transforming publics. American teenagers are increasingly engaging in networked publics and, as such, the properties and dynamics described in this chapter shape the everyday practices of these teens. While these dynamics have affected different niche audiences before, the popularity of social network sites among teens introduced these dynamics to a broad cohort. When participating in networked publics as a matter of everyday life, teens are forced to face these dynamics and the potential complications brought on by them, especially with regard to identity work, peer sociality, and negotiating those who 
hold power over them. Not only are teens adjusting to a public life shaped by these dynamics, but, through their active engagement, they are also developing strategies to manage the complexities and are reworking both the technology and social norms as a response to structural shifts. Their practices and strategies reveal ways in which people, technology, and society adjust to and reconfigure the structural conditions of any given environment. 


\section{Chapter 2: Choose Your Own Ethnography}

Teenagers have been active participants in networked publics since the early days of Usenet, but their participation has been poorly documented. I was a member of that early cohort of geeky kids who found freedom and agency through online interactions in the 1990s. After spending my middle-school years desperately trying to be liked by the popular kids, I relished the opportunity to connect with people who shared my interests and geeky tendencies, regardless of how old they were or where they lived. There were teen-only spaces online, but teens were also participating alongside adults in interest-driven communities without disclosing their age. For socially ostracized teens, including myself, the Internet afforded an opportunity to be taken seriously-it seemed that what we had to say mattered far more than how old we were. In 1993, The New Yorker published a cartoon with the caption, "On the Internet, nobody knows you're a dog" (Steiner 1993). For many of my peers, that cartoon may well have said, "On the Internet, nobody knows you're a teen." Those of us who did not fit in at high school relished any possibility that our ages would not matter if we were articulate when we typed out our thoughts.

While the Internet shaped my teen years and those of many of my peers, little is documented about early teen involvement in the Internet. Early scholarship and journalism mention teens and age in passing (Cherny 1999; Kendall 2002;

Rheingold 1994), but the actual practices of early adopter teens were relatively 
invisible and poorly documented. Teens are implicated in early cybertheory, especially with respect to the ways in which technology could provide tremendous opportunities for youth to work out identity puzzles (Turkle 1984, 1995) and for youth to escape the trappings of age. At that time, early participants-both teen and adult-often embraced an idealistic vision of the Internet as a personal liberator (Barlow 1996; Rheingold 1994; Turner 2006). Teens, in particular, had good reasons to view it as such; for the first time, they were an authority on something that their parents did not understand (Tapscott 1998: 36-38). Scholars began positing that technology would free people from the constraints of their bodies, altering the makings of identity (Haraway 1991b; Stone 1995; Turkle 1995). Although technology did not obliterate embodied identity, it did obfuscate teen practices for a while.

By the time scholars began focusing on teens' online practices and the ways in which they incorporated the Internet into their everyday lives (Holloway and Valentine 2003; Lenhart et al. 2001; Livingstone 2002), much had changed. Internet adoption had skyrocketed and the majority of American teens had gone online (Lenhart et al. 2001). Instant messaging had taken off and blogging was under way. Adults fretted about teen engagement with the Internet. The e-commerce bubble had boomed and burst. Laws had emerged to regulate the Internet. In short, the Internet that teens experienced in 2001-2003 did not resemble what I had grown up with. Both technology and society had changed. 
These dynamics shaped my dissertation project. I had grown up online and was tremendously curious to know what being a teen with Internet access meant today. General scholarship concerning mediated participation had swung from cyberutopian theory to quantitative analyses of adoption practices; ethnographies examined a plethora of different mediated gen res and online communities. In devising this project, I was driven by the desire to do what I wished scholars before me had done. I wanted to know what was normative when it came to American teens and social media. I wanted to get beyond the statistical depictions of teen Internet use and understand the cultural logic behind their mediated practices.

In order to examine everyday practices of American teens, I decided to embark on an ethnographic study of the role of networked publics in the lives of American high-school teenagers, focusing on how teens incorporated popular forms of social media into their everyday practices and experiences. My ongoing interest in identity, privacy, impression management, and social interaction shaped where I began, but my fieldwork also led me to explore other topics. While I had grown up at a particular point in the history of the Internet, it was tremendously rewarding to be able to watch a new generation of teenagers embrace an entirely different set of circumstances and technologies.

I was well positioned to watch the phenomenon of teen engagement with social network sites unfold and I used that to my advantage. MySpace became popular with teens just as I began my fieldwork. I did not initially intend to focus on social 
network sites, but as teens turned their attention to these sites, so did I. Additionally, I had just finished a project studying the 20-30-something early adopters of an early social network site called Friendster (boyd 2008b). I was also tracking a plethora of social network sites, including MySpace and Facebook. I was familiar with the technology as well as the business behind it. Growing up online, I was comfortable with the rise and spread of new genres of social media and had developed a cautious and critical eye for wading through the hype that inevitably comes with such phenomena.

While ethnographically studying a sociotechnical phenomenon was thrilling, it also created various methodological challenges. I struggled to find a field site. Rather than focusing on one specific site, I developed a multisited project in which my field site became a network of different sites. I moved between mediated and unmediated spaces to observe and interview teens. This too created complications in tems of visibility, access, and privacy. I faced institutional barriers, such as navigating human subjects approval for my research, a process meant to guarantee my research would cause no harm to those I studied. Studying the technology itself was far easier than studying the practices that took place, which again was easier than understanding the context in which those practices were situated. Ethnographically, I struggled to overcome structural and social boundaries and get deep into the lives of teens. Finally, because the phenomenon that I was studying was mired in all sorts of controversy, I struggled to stay focused on teen practices and not become distracted by the hype or fears that surrounded what I was studying. 
This chapter documents my methodological approach and data. Additionally, I outline key struggles in my methodological trajectory, articulating decisions that I made, noting bumps and bruises, and highlighting the lessons I learned along the way.

\subsection{Ethnography in Context}

I approached this project as an ethnographic study because this approach allowed me to make sense of cultural practices in the context of everyday life. I see ethnography as a descriptive account of cultural practices, grounded in data attained through ethnographic fieldwork and situated in conversation with broader theoretical frameworks. Ethnography has its roots most formally in cultural anthropology (Clifford and Marcus 1986; Geertz 1973; Malinowski 1922; Marcus 1998; Mead 1928), although scholars from a variety of fields have begun adapting the method to engage with other theoretical frameworks. I approach ethnography as an interdisciplinary scholar and set my fieldwork against multiple theoretical backdrops.

While approaches vary, ethnographic fieldwork relies on participant observation, qualitative interviews, and analysis of cultural artifacts to make sense of cultural practices on their own terms. Ethnographers use different techniques to interpret, complicate, and analyze cultural practices, situate complex cultural phenomena, and map social worlds from the bottom up. As a method, ethnography does not speak to individual traits or beliefs but to the complexity and interconnectedness of 
culturally driven practices and norms. Ethnography produces a topological map of a particular set of cultural practices. My interest in mapping out the practices and cultural dynamics of American teens led me to ethnography.

Ethnographic methods are constantly evolving and even more frequently debated as researchers challenge once ubiquitous practices in response to fluctuating research norms and understandings. For example, early ethnographers bounded cultures by geography and identity while current ethnographers argue that global systems are at play (Appadurai 1996) and that even supposedly remote cultures are shaped by outside forces (Piot 1999). Even commonly used ethnographic terms can be destabilized. For example, Appadurai (1996: 11-16) argues that using the term "culture" as a noun conceals and decontextualizes the diversity of cultural practices that exist. Needless to say, many aspects of ethnography, its use, and its referents are contested. This makes locating ethnography or ethnographic practices challenging.

While methodological debates concerning ethnography are vast, I want to focus most directly on those studies and debates that specifically concern ethnographic studies of Internet-enabled practices before outlining my methodological choices. The unique challenges involved in Internet ethnography play a significant role in shaping my approach. 


\subsubsection{Ethnography and the Internet}

Ethnography has long been used to understand online communities and mediated practices. Some early examples include Elizabeth Reid's (1991) examination of IRC and Nancy Baym's (1993) study of the community and practices in a Usenet newsgroup about soap operas. While humanists began mapping out a vision of cyberculture (Haraway 1991b; Stone 1995), ethnographers focused on cultural practices under way. Much of cybertheory was branded technoutopian by ethnographers because it focused on how the Internet could free people from their corporeal limitations (Stone 1995), their social restraints (Turkle 1995), and the political regimes that regulated them (Barlow 1996). Ethnographers argued that such visions might be a guiding light, but they did not reflect how people actually experienced mediated life. Lori Kendall (2002) documented how people who engaged in online forums create online representations that bear close resemblance to their offline selves; she showed how social constructs like gender are reproduced and reinforced online. Jenny Sundén (2003) further challenged the disembodiment rhetoric by showing how the body plays a crucial role in people's mediated experiences. She argued that neither "postmodern utopianism (the online world as disengaged from the physical)" nor "realistic determinism (the online world as a copy of the 'real')" quite get at the ways in which online identities extend the physical while also being configured by the digital (Sundén 2003: 109). Kendall and Sundén-and their contemporaries_acknowledge that mediating technologies alter 
the role of bodies and identity, but they challenge early cyber-utopian visions of the Internet as providing freedom from traditional physical constraints.

As ethnographies of mediated practices emerged, scholars began struggling with the relationship between online and offline contexts, both theoretically and methodologically. Some viewed the Internet as a new kind of "third place" with its own cultural dynamics (Bruckman and Resnick 1995; Soukup 2006), while others saw the Internet as a tool that inflected everyday life in interesting ways (Haythornthwaite and Wellman 2002). Christine Hine (1998: 14-40) articulated this best when she explained that the Internet can be understood as both a culture and a cultural artifact. As a culture, the Internet possesses a set of norms and practices that are unique and that should be studied separately from unmediated life. As a cultural artifact, the Internet exists within the broader cultural context in which people live. Which approach ethnographers take fundamentally shapes their fieldwork and analyses. This divide is one of the core concerns underlying Internet ethnography and raises all sorts of methodological questions about boundaries, privacy, and ethics (Buchanan 2004; Markham and Baym 2008).

Much of what early ethnographers of the Internet grappled with concerned the idea of whether the Internet was or was not a separate space with a culture of its own. If discussed at all, unmediated life was invoked when scholars questioned the ways in which the "virtual" was like or unlike the offline. While this approach has value, there was very little discussion of how mediated and unmediated interactions 
are often seamless. Early on, ethnographers began challenging this approach by emphasizing the importance of taking context into consideration, even when doing studies of online communities (Kendall 2002). Others began highlighting the continuities between what might be seen as online and offline contexts (Bennett 2004; Miller and Slater 2000).

To methodologically account for the relationship between online and offline practices, ethnographers began "tracing the flows of objects, texts, and bodies" as they moved between mediated and unmediated environments (Leander and McKim 2003: 211). Some scholars collected and analyzed both online and offline data (Haythornthwaite and Wellman 2002; Orgad 2008). Others emphasized interactions or communities and followed the relationships between people and activities as they moved between online and offline environments (Hodkinson 2002; Kelty 2008; Wilson 2006). Ethnographers studying subcultures, social phenomena, and communities of practice incorporated multisited fieldwork techniques into their methodologies so as to trace activities that took place in different mediated and unmediated environments. Even those who focused specifically on mediated practices found that tracing secondary, unmediated interactions enhanced their research. For example, T. L. Taylor (2006) joined gamers for in-person meetups to get a better understanding of their mediated dynamics. Ethnographic studies of mediated cultural practices increasingly moved among different field sites and devised different techniques for following peoples, practices, and communities across different mediascapes. 
The interplay between online and offline peoples and practices also led scholars to focus on the ways in which the cultural foundations of mediated and unmediated environments are co-constructed. From Nina Wakeford's (2003) study of how local cultural dynamics are embedded in global communication through London Internet cafés to Mizuko Ito’s (2005) discussion of the mobile phone as a “technosocial tethering," ethnographers examined how technological practices are influenced by cultural practices, physical place, and the ways in which geographically determined cultural practices configure technologies. In other words, as technological systems are socially constructed through usage (Bijker et al. 1987), the sociotechnical practices that emerge shape the cultural landscape of both mediated and unmediated environments.

As more ethnographers interested in the Internet began employing multisited and social constructionist approaches, the claim that the Internet was a culture in and of itself began to fade and few protested its disappearance. This issue reemerged for debate in 2008 when Tom Boellstoroff (2008) defended his decision to locate his fieldwork wholly inside Second Life, an immersive virtual world. Boellstoroff argued that it is critical to study virtual worlds in their own right as a culture with their own context and that "to demand that ethnographic research always incorporate meeting residents in the actual world for 'context' presumes that virtual worlds are not themselves contexts" (Boellstorff 2008: 61). While he acknowledges that some questions require multisited fieldwork, he challenges the assumption that 
unmediated practices inform mediated ones. His challenge forced me to reflect on my own assumptions.

Boellstoroff s decision to bound his fieldwork by virtual interactions is driven by his claim that most Second Life participants do not interact with one another outside of the virtual environment. His argument is that Second Life is a contained culture because people's only interactions with one another take place there. As I worked out why I found this argument disturbing, I faced two issues. First, I questioned his claim. Other accounts of Second Life detail people's finding partners and friends online whom they connect with offline (Au 2008). Furthermore, in many online environments, people regularly connect with people they meet across a variety of Internet contexts (Parks and Floyd 1996; Rheingold 1994, 2002; Taylor 2006) and, more commonly, they interact online with people whom they know from unmediated environments (Ellison et al. 2007). I was not convinced that Second Life was unique and truly self-contained. My second concern was more philosophical. Ethnographers studying unmediated cultural practices had started to shift away from bounding projects by geographic proximity, emphasizing that global and networked forces shape even supposedly local cultural dynamics (Appadurai 1996; Piot 1999). Clifford (1997) argued that ethnography must adapt to the ways in which cultures are not isolated. Given that ethnographers looking at small villages had moved away from seeing these communities as bounded cultures, I failed to understand how a community that explicitly defined itself as people's second lives could be examined without attention to those people's "first" lives. 
While I disagree with Boellstoroff's approach, his critiques of the way in which online/offline fieldwork implicitly prioritizes the offline are valuable. Offline fieldwork should not simply be a mechanism to assess if people's mediated representations of themselves are accurate or to understand how lived, gendered, and raced experiences of everyday life affect mediated practices. The offline is not simply the "backstage" to the mediated "front stage." Rather, different geographically and socially constructed contexts are entwined. Conversations and experience move among media, space, and time. I agree with Boellstoroff that we must take the experiences people have in mediated environments seriously and understand how that context shapes cultural practice. Yet we do ourselves a disservice if we bound our fieldwork by spatial structures — physical or digital—when people move seamlessly between these spaces. Both mediated and unmediated fieldwork should have as their goal a rich understanding of the networks of people, objects, and practices.

\subsubsection{Networked Ethnography}

Multisited fieldwork is increasingly common in ethnography (Green 1999; Marcus 1995), but there is no consistent framework for relating the different sites to one another. One approach, articulated by Jenna Burrell (Forthcoming), is that field sites should be defined and viewed as a "network composed of fixed and moving points including spaces, people, and objects." She argues that such a framework allows ethnographers to approach social phenomena as a continuous system and 
capture the coherence and fluidity of the different spaces people occupy. Burrell's network-driven approach offers one way of addressing Arjun Appadurai's (1996) call for scholars to look at the fluid dynamics of "cultural flows" that takes place in a plethora of different "scapes" (e.g., ethnoscapes, mediascapes, technoscapes, financescapes, and ideoscapes). Both Burrell and Appadurai acknowledge that mediated landscapes disrupt traditional ideas of spatiality and that this requires rethinking how ethnographers should traverse such spaces.

Focusing on Burrell's networks or Appadurai's scapes addresses Boellstoroff's concern that multisited ethnographic studies implicitly privilege one site over another. Rather than starting with one environment and moving to the other or constructing a multisited project from disconnected sites, a network-driven approach should allow scholars to fluidly move along axes of people, places, and objects, generating meaningful networks and scapes. The result is indeed multisited, but the sites are chosen in relation to one another. Each axis constitutes a network in its own right, generating a field site that is a network of networks.

As Burrell articulates, approaching a field site as a network involves finding different entry points into a phenomenon, following different relationships between people and practices, and making sense of different types of networks and their relation to one another. Most important, it requires considering relationships among people, spaces, and objects, as opposed to studying these in isolation. While 
this approach ruptures any traditional notion of boundaries, it helps ethnographers track, study, and understand phenomena that are constantly moving.

\subsection{My Field Site in and of Networks}

Instead of starting with one bounded site, I decided to approach my field site as a network. I focused my study on the intersection of American teenagers, their relationship to networked publics, and, in particular, the sociotechnical phenomenon marked by the rise of social network sites. I began my fieldwork from different angles and traversed the phenomenon using different approaches. My fieldwork includes mediated and unmediated environments and I moved across different social contexts and engaged with different relevant social groups to gain an understanding of what was taking place. Approaching this puzzle, I began broadly and narrowed my focus as I achieved clarity. As appropriate, I expanded my scope when following specific people or trying to make sense of specific spaces. This created many layers of awareness that allowed me to locate people, spaces, and practices in a broader context.

I want to focus on three conceptual structures that shaped my field site: youth, networked publics, and the United States. Brought together, these three formed a complex intersection of people and practices. With each, I started broadly and was forced to confront issues of boundaries as I made decisions about where to focus. These three structures-representing people, mediated space, and unmediated geography - were constantly entangled. Together, they allowed me a variety of 
starting points for understanding the phenomenon I was studying. This was especially important when I came up against ethical or structural limitations.

Through the combination of narrowing and conscious decision making, my fieldwork centered on American teenagers and their engagement with networked publics. While my field sites primarily consist of three dominant and overlapping networks, my fieldwork also includes a variety of peripheral networks that involve the people, spaces, and cultural artifacts that surround teens. Parents, teachers, and youth ministers all became important in their relation to teens. Likewise, schools, camps, malls, homes, and networked publics all became important spaces, and technology, fashion, and media all became crucial cultural artifacts.

\subsubsection{From Youth to American Teenagers}

Choosing to focus on American teenagers was a conscious act of boundary making. Initially, my focus was more broadly on "youth.” Prior research on early adopters and subcultures (boyd 2008b) had led me to think through the construction of youth culture. Yet I quickly found the label "youth" too unwieldy for this project. The term itself lacks clarity-is it defined by age (e.g., anyone over 14 and under 24), legal standing (e.g., "minors”), life stage (e.g., unmarried individuals with no children), or something else? Literature on childhood, teenagers, youth, adolescence, minors, and students only complicated matters. More important, the population marked by such a label is too diverse for analysis. 
I chose to exclude those under 13 from my purview simply because the vast majority of websites do. Compliance with the Children's Online Privacy Protection Act (COPPA) requires companies that collect personal data (e.g., a log-in) to get parental consent for those under 13. Rather than devote resources to collecting parental consent, most companies require users to be 13 and older. While I knew that children under 13 lie about their age to gain access to various sites, I decided to focus my attention on those who could legitimately participate to avoid introducing an additional confounding variable.

Because my project was shaped by the social network site phenomenon, another natural boundary emerged. Initially, Facebook allowed only college students to join and their practices were often different from those of youth participating on MySpace. Many differences appeared to be shaped by broader structural conditions each group faced. Parental rules and legal restrictions like curfew laws and driving age tended to play a significant role in the choices made by high school-age teens, even if only as something to rebel against. Parents played a less direct role in controlling the practices of older youth. Older youth appeared more likely to leave home or at least have more flexibility concerning their mobility and choices. While most high school-age teens attend high school and live with their parents, older youth's lifestyle and practices are more variable. Some attend college while others are in the military or working. Some live at home while others have moved away from their parents' places. The structural forces that configure older youth's lives are much more varied. By focusing on high school-age teens, I could look at mediated 
dynamics in relation to relatively stable social structures like parents and school while still leaving room to talk with those who had decided to unenroll from high school before graduating, those who had run away from home, and those who were living in nontraditional housing arrangements.

Narrowing my focus by a combination of age and life stage forced me to reconsider what I meant by "youth." While "youth" seemed too broad a descriptor, I struggled to find a term that properly captured the cohort that I had conceptually bounded. I rejected "students" for three reasons: (1) not all high school-age youth are students; (2) not all students are high-school age; and (3) I did not want a term that referenced a role to a system that I was not studying. The legal concept of "minor" puts youth in relation to adults while also failing to accurately describe the population. "Children" is problematic for the same reasons. The term "adolescent" is often used to negate youth agency by suggesting that they are at an immature psychological stage at which they cannot be trusted to make decisions.

I am not the first scholar to struggle with this linguistic issue. Barrie Thorne (1993) conscientiously opted to use the term "kids" because that was the language her informants used. I decided against this term because it is not native to high school-age youth and because it typically signifies a population younger than the cohort I was studying. I finally decided to use the term "teenager" or "teen." While technically not all teenagers are of high-school age, most are. The term certainly has its problems - not the least of which have to do with the evolution of this term as a 
marketing construct in the 1940s (Hine 1999)—but more than any other term, I thought "teenager" encapsulated the population I was observing.

For entirely practical reasons, I decided to focus only on those teens who spoke English. I conducted all of my interviews in English and, while I observed nonEnglish online content, I analyzed only that which was in English.

To analyze mediated practices, I decided to exclude the small minority of teens who lacked any form of Internet access. In late 2006, Pew found that 93 percent of U.S. teenagers ages 12-17 had some form of Internet access (Lenhart et al. 2007). While I wanted to exclude teens with no Internet access, I did not want to exclude teens whose access was limited or those who were unable to access social media because of parental restrictions or school filters. During my fieldwork, I did not meet a single teen who completely lacked Internet access, although I interviewed and observed many teens who were unable to access the Internet from home or who had only limited, filtered access in schools and public places or whose parents tightly restricted their access. Given the availability of access in schools and public places at all socioeconomic levels, I began to suspect that those with zero access primarily lived in rural regions, were homeschooled, or were otherwise not part of contemporary teen culture and communities.

Because I wanted to interview teens in person, I decided to add a geographic filter to my scope. The Internet allowed me to observe teens from around the world, but a global scope was impractical and unmanageable. I considered locating my 
fieldwork in Los Angeles, but I decided that would be the wrong scale. I was too interested in understanding how practices varied across the United States. I decided to sample across the United States both online and in person. At some levels, the United States is a coherent cultural frame, while at others it is not at all. Media, consumerism, and politics all shape the country in fairly consistent ways. Clear Channel pumps a consistent selection of music to more than 1,200 radio stations in the United States; cable TV shows the same popular shows to every region; and movie theaters promote the same blockbusters. Chain stores and franchises dominate the geographic landscape and it is difficult to drive far in this country without finding a Starbucks, Wal-Mart, or McDonald's. Schools are shaped by "No Child Left Behind" standards, and, to the degree that people pay attention, what happens in D.C. defines the political landscape. But even taking into account demographics, there are huge cultural variations across the United States. The experiences of a white Christian teen from a poor family in New Mexico look vastly different from those of an equivalent teen in Appalachia. The same stores and food may exist in St. George, Utah, and Okemos, Michigan, but those small towns are worlds apart. The United States began as a loosely connected network of distinct communities with separate cultural dynamics and, even as a large nation-state, its communities still retain much of their unique flavor.

In deciding to take on the United States as a geographic frame, I made a conscious decision to work through cultural similarities and differences that are inherently a part of this country. I intentionally use the cultural referent 
"American" instead of the nation-state term "United States" to emphasize the complex cultural dynamics that are linked to the former. The United States is a nation-state defined by its (theoretically impermeable) borders and (often problematic) political activities. Conversely, an American identity is about the intersection of common cultural forces, geographically diverse cultural practices, and proud individualism marked by sentimental notions of freedom. As I see it, part of what it means to be an American is to constantly shape and be shaped by the tornado of cultural forces that constantly flow through the United States, resulting in a different and far more complex cultural experience than the image of American culture that the United States tends to export through media and commerce.

\subsubsection{From Networked Publics to MySpace and Facebook}

A networked public is both the space constructed through networked technologies and the people who are connected by those technologies. Genres of social media that produce spaces for publics to emerge are themselves networked publics. Although the most popular forms of social media are primarily about connecting individuals or small groups (e.g., instant messaging, text messaging), networked publics capture the social imagination by suggesting an alternative organization of society. Usenet, chatrooms, the blogosphere, and social network sites are all networked publics that allow people to gather, share, and converse. The structure of these spaces suggests that people can connect with others like them across social and geographic divisions. This, in fact, is the crux of the techno-utopian 
dream. My interest in networked publics stems from a fascination with the differences between what is imagined as possible and what people actually do when they gather in and create public spaces online.

My project focused broadly on networked publics. When I began, two blog/journal communities_Xanga and LiveJournal—were the most popular mediated spaces in which teens gathered. As teens grew enamored with MySpace and, later, Facebook, I followed them. Quickly, MySpace and Facebook played a much more central role in teens' lives than Xanga and LiveJournal ever did. MySpace and Facebook became the dominant mediated site for my fieldwork simply because they dominated teens' attention.

Temporally, my fieldwork traced the rise of MySpace. When I began observing youth practice in late 2004, only a handful of teens had adopted MySpace, but it quickly became the most popular networked public space in which American teens gathered. Midway through my fieldwork, Facebook started to take off and capture the imagination of American teens. I followed this phenomenon as well. By the end of my fieldwork, Facebook had become a significant competitor to MySpace and MySpace's status among teens had begun to fade.

MySpace and Facebook both host profiles for millions of American teenagers. It is impossible to follow every teen who uses these sites so I developed strategies for following different cultural dynamics that took place without following all participants. On MySpace, I used a random sample of user profiles as entry points 
and followed the Friend networks to get a sense of the topology. Because MySpace's profiles are linked to numerical user IDs, I was able to use a random-number generator to select a subset of profiles to view. Some of these had been deleted and others were bands-I ignored these. With the help of MySpace, I viewed the rest and analyzed all that were most likely teenagers. I did not keep quantitative measures of those that I discarded, as my goal was purely to get a random sample of teen profiles.

Because of differences in structure and access, it was not possible to get a random sample of Facebook profiles. Instead, I made accounts in different regional networks and started with a sample of teens who made their profiles visible to these networks. I could view their Friends' lists, but I could often not view their Friends' profiles. My sample on Facebook was very limited and unrepresentative but, at the time, I was less concerned with Facebook profiles because the options for selfexpression were more limited and fewer teens were engaged there. Only at the end of my fieldwork did I come to consider my lack of access as a limiting factor.

As a participant on these sites, I was connected to friends, colleagues, and peers; this participation provided an entirely different angle for understanding these sites. As a member of different technology circles, I also had access to the business side of these companies, providing yet another vantage point. While these sites became a core focus of my fieldwork, challenges in collecting data also complicated my work. The semi-public nature of networked publics gave me a window into teens' lives, but 
it also taught me that the limited view is often misleading. As I will discuss later, access and visibility became core methodological challenges.

Throughout my fieldwork, I tracked other forms of social media and networked publics. I kept tabs on older genres that were once fairly popular with teens, including chatrooms and blogs. I followed media-sharing services as communities began to form around them. Yet while sites like YouTube gained tremendous popularity among youth, teens predominantly used them as content-access and distribution tools rather than networked publics. Many pundits argued that the next teen fad would be immersive 3D worlds like Second Life and massively multiplayer online role-playing games like World of Warcraft, but no mass adoption took place while I was doing fieldwork. I observed teen practice in and interviewed them about a wide array of networked publics, but MySpace and Facebook were the stable crux of teen participation.

MySpace and Facebook serve as crucial case studies throughout my dissertation. ${ }^{1}$ MySpace and Facebook allowed me to observe traces of a variety of teens and their peers in a highly mediated environment. These tools gave me a window into aspects of many teens' lives, but the picture was always partial and slanted. While there are significant limitations to what I could watch through MySpace and Facebook, these sites allowed me to observe teens from all over the United States. Such access

\footnotetext{
${ }^{1}$ Chapter 3 documents the history of these sites and places them into a broader context of social media; Appendix 2 details specific site features so that readers can follow the discussion even if they are not familiar with the specific sites.
} 
provided valuable insights into the cultural dynamics of specific places, which, in turn, helped me as I began interviewing throughout the country.

\subsubsection{From the United States to Teens' Homes and IHOP}

While observing teens in Montana and Texas through MySpace did not require me to leave my chair, moving between different physical locations was time consuming and economically costly. When I began interviewing teens in person, I had to take geography into account in ways that I never considered online. The third core component of my field site-the United States—comprised a diverse set of individual communities in which I spent time visiting and working. I intentionally chose a variety of different types of communities in different regions and aimed to interact with a diverse swath of teens.

In choosing where to visit, I leveraged my travel schedule to get to different regions and then aimed for diverse types of communities. In 2006-2007, I extended work-related trips around the country to interview and observe teens in the surrounding areas. For logistical reasons, I decided to focus on teenagers living in urban, suburban, and small-town regions, although I did end up speaking with and observing a handful of teenagers living in rural settings.

I spent more than 150 days outside of California doing fieldwork during the 2.5 years I was in the field. My time in California was split between San Francisco and Los Angeles. I interviewed 94 teenagers and observed hundreds more in the District 
of Columbia and 16 states: California, Colorado, Connecticut, Iowa, Kansas, Massachusetts, Michigan, Missouri, Nebraska, New Jersey, New Mexico, New York, North Carolina, Pennsylvania, Texas, and Washington. I visited cities, small towns, new suburban communities, and old suburbia. I spent some time in rural Iowa and Kansas, but I primarily focused on nonrural environments. Unfortunately, a planned trip to Georgia was cancelled, leaving a visible hole in regions from which I interviewed and observed teens. In 2008, I had the opportunity to talk informally with teens in Louisiana and Utah, but I did not arrange interviews or structure our conversations based on my research. Still, in both environments, teens talked with me about their online practices after they learned that I was writing about this topic.

In each community, I practiced a combination of interviewing and observing. I observed social dynamics in shopping centers and malls, movie theater lobbies, fastfood restaurants, cafés, and churches. I watched teens socialize with peers at the beach, at all-ages music venues, on buses, in parking lots, and at youth centers. I visited high schools and after-school programs in Northern and Southern California, New Jersey, and Massachusetts. In short, I tried to spend time in all of the semipublic physical places where teens gather. I also visited more adult-centered environments and spoke with local community members about teen culture.

In most of the communities, I never stayed long enough to have a local's perspective. Yet even in those towns where I do or did live-Lancaster, 
Pennsylvania; Boston; San Francisco; Los Angeles-teens gave me an entirely different perspective on towns that I knew fairly well.

By spending time in multiple geographically organized communities in the United States, I was able to see teens' lives through a vantage point that encompassed differences across communities. For ethical reasons that I will discuss later, I was not able to directly communicate with teens online. Thus, in-person interviews became essential for mapping out teens' logic behind their practices.

\subsubsection{Locating Myself in My Field Site}

In approaching this project, I was conscious to account for my own experiences in relation to who, what, and where I was studying. My identity and experiences shape this project, my interviews, and my analysis. I am a 30-year-old, white, American-born, college-educated, queer woman. I have lived in eight different communities in the United States, including small towns, suburbs, and big cities, and I have spent time in many more. Because I am familiar with the United States, I worked diligently to interact with cultural artifacts and observe cultural practices that were unfamiliar to me.

I have also been online since I was 13 and the Internet has shaped my life and identity in uncountable ways. I am frequently called a "digital native," a controversial term applied to some of today's teens who incorporate mediating technologies in every aspect of their lives (Palfrey and Gasser 2008). My media and 
technology usage is far more aligned with that of today's teens than with that of most adults. That said, I am no longer a teenager and I do not share many of teens' concerns, practices, routines, and lifestyles. I use many of the same tools, but I do so differently.

My experience as a teenager, my relationship to social media, and my comfort with the United States put me in a unique position entering the field. Conscious of this, I constantly reflected on my assumptions and expectations. Throughout my fieldwork, the mantra of "make the familiar strange" echoed through my head.

\subsubsection{An Imperfect Field Site}

The value of building a field site as a set of networks is the opportunity for continuity, one of the advantages Burrell (Forthcoming) documents for choosing to approach fieldwork this way. Creating continuity requires being able to move seamlessly between different mediated and unmediated environments. The fluidity with which teens move between different contexts highlights the continuity that they experience. When I began my project, I believed that it would be possible to follow them across these spaces. Yet social, cultural, and ethical limitations thwarted my intention to move along the different axes I laid out. In essence, I struggled to handle the complexities of a collapse in contexts as a member of the invisible audience, issues that I laid out in Chapter 1. 
As minors, teens are vulnerable subjects. Part of their vulnerability stems from general concerns about the power relations between adults and minors. Cultural fears about sexual predators, stalkers, and abductors influence what is socially appropriate and ethical. Furthermore, parental consent is necessary for direct engagement with teenagers, which means that parents must preapprove any situation in which I can interact with teens. With parental permission, I was able to interview teens in predefined settings, but I could not simply follow teens about their daily lives or to school, even if teens gave me permission to do so. This restriction obliterates any chance of natural fluidity. In theory, following teens when they gathered with their friends in public settings was possible but felt stalkerish, as would following them beyond those settings. In short, my position as an adult meant that there was no comfortable way to move seamlessly across unmediated contexts without triggering ethical alarms.

Moving between mediated and unmediated environments introduced different challenges. While teens who I interviewed frequently showed me their online profiles, I was not able to sit with them on an average night when they were socializing with their friends through the sites. I accepted all Friend requests from teens, but I thought asking teens to be my Friend was an abuse of authority. As such, I could not really participate in collective "hanging out digitally" either. I could observe teens whom I interviewed, but this was not the same as creating a continuous space for interaction. I knew that what I was seeing included in-jokes, references to offline activities, and conversations that had begun elsewhere, yet I 
could not follow the referents. While my access was in some ways limited in these spaces, I was also privileged to have access to more everyday teen banter than I would normally encounter given my adult status.

Approaching teens online for offline interviews or interactions felt inappropriate given the cultural context concerning teens and online strangers. While scholars studying other communities felt comfortable contacting people online and interviewing them there or meeting with them in person (Baym 1993; Rettberg 2008; Taylor 2006), they were primarily dealing with adults. Mass media, safety organizations, police officers, and parents regularly tell kids that they should not talk to strangers online because they are potential child predators. Although, as I will discuss in Chapter 6, these fears are overblown, they are nonetheless real. As a result, I thought trying to move from online to offline would be inappropriate.

In unmediated contexts, observing typically makes a researcher visible to those being observed. Online, this is not the case. While I was an active participantobserver in networked publics, I was practically invisible to teens. I had a blog and profiles on both MySpace and Facebook long before any of the teens I met did, ${ }^{2}$ but there was no reason why teens should read my blog or stumble on my profile. Simply having a profile and being an active participant among my own friends did not make me visible to the teens I was observing. When I visited teens' profiles,

\footnotetext{
${ }^{2}$ I began blogging in 1997. I created my MySpace account in September 2003 and my Facebook account in mid-2004. Additionally, I began studying social network sites in the form of Friendster in early 2003 and created a profile on most major social network sites before I began studying them.
} 
they had no way of knowing that there were visitors, let alone who I was. Most teens I observed were (and still are) completely unaware of my existence.

As discussed in greater detail in Chapter 4, there is no implicit visibility online. Without my making my presence explicit, there is no way for someone to know that I am there. Yet there are few acceptable ways to make my presence known. Ethnographers in other networked publics often make a point of "de-lurking" in online communities to make their presence known. In communities where chatting with strangers or leaving comments is socially acceptable, de-lurking is often valued. Researchers entering such spaces can make their presence known and become trusted participant-observers. Such interactions allow them to develop social rapport with those they are observing and even contact participants directly for further conversation (e.g., Baym 1993; Rettberg 2008; Taylor 2006). This is not the dynamic of teens and social network sites. By and large, teens talk to people they know and have little interest in developing connections with strangers. To make my presence known, I would have had to initiate explicit contact with teens. I could have sent teens private messages, added them as Friends, or poked them on Facebook. Such direct contact removed from any social context in which it is socially appropriate feels unethical, not to mention the challenges associated with an adult's contacting a minor. I was not innately visible nor could I make my visibility known without direct contact. 
While unsolicited messages to teens felt inappropriate, I initially thought that I could leverage connections I had to contact other teens. I decided against this approach because of the potential it had to put teens in an awkward situation, particularly when they were forced to weigh their friends' support against their parents' warnings. Such a dynamic is best exemplified by an encounter I experienced in late 2006.

I met Dan, a 15-year-old from Northern California, at a conference for geeks. We talked about a variety of things and, at the end of the conference, our conversation spilled into an email dialogue. He invited me to be his Friend on MySpace and we kept in touch. At one point, we started talking about copying and pasting code into MySpace and he told me about Cory, a friend of his from school whom he thought to be the expert on the matter. He told me which of his Friends on MySpace was Cory and encouraged me to contact him. I sent Cory a MySpace message, indicating that Dan had encouraged me to contact him and explaining that I was a researcher. I then asked him a question about his technical practice. Cory responded with a curt note that included a brief explanation and a message about how his parents did not want him talking to strange adults online and while I seemed to be who I said I was, I would need to call his parents for permission to continue the conversation. I felt dreadful and apologized profusely for putting him an awkward situation. When I recounted this story to Dan months later, he sighed and acknowledged that concern about online strangers was rampant. My encounter 
with Cory made me realize that I risked making teens very uncomfortable by contacting them online for online-only conversations, let alone offline encounters.

Creating continuous spaces across different media requires a certain degree of permeability between those spaces. The potential permeability of different contexts terrifies parents. I erred on the safe side and chose to interact with teens one context at a time. As a result, I experienced teens' lives in a staccato fashion. I observed teens in discrete, bounded spatial contexts, but I was unable to follow them across spatial dimensions as they moved. That said, in casting my net widely, I was able to get tremendous information from multiple discrete contexts. Each interaction provided valuable perspectives into the lives of teens and the spaces that they inhabit.

\subsection{My Data}

The data that shaped this project stemmed from a variety of different sources, collected during an extended period. Formally, my ethnographic data collection took place during a period of 2.5 years, starting in early 2005. Informally, it began earlier and continues to this day. The bulk of data used in my analysis consists of digital content captured in networked publics and semi-structured in-person interviews. Both online and offline observation data also shape my description and analysis, as does material attained through a wide array of disparate sources. For example, I also collected data in informal settings and was able to access data 
collected by others. What follows is a description of the different types of data that I acquired, used, or had access to as a part of my fieldwork.

By the time this research project formally took shape in 2005, I was already immersed in the social network site phenomenon and looking at teen and young adults' participation in networked publics. For a different project, I began taking fieldnotes on social network sites in March 2003 and began taking fieldnotes on MySpace in January 2004. During this period, I focused broadly on early adopters. I also began taking fieldnotes on teen and young adults' blogging in July 2004. I started observing teen practices on MySpace in early 2005, but I did not begin unmediated observations of teens in public spaces until after receiving human subjects approval in May 2006. I began a series of formal semi-structured interviews in December 2006, although I had had numerous information conversations with teenagers before that. I left the field to start writing in October 2007. That said, I am still immersed in the social network sites and I still regularly interact with teenagers in unmediated contexts.

\subsubsection{Online Data and Observation}

As part of my data collection, I spent extensive time online. I never kept records of how much time I spent surfing profiles, reading teens' blogs and news articles about teens, informally talking with a variety of people, observing teenagers online and offline, or tracking the phenomenon more broadly. I was embedded in the online phenomenon involving teens and social network sites from mid-2005 to 
mid-2007 (with a three-month break in spring 2006). When I was involved in fulltime data collection, I probably spent roughly 50 hours a week inside teen networked publics or observing teens offline. I estimate that I spent more than 2,000 hours surfing MySpace alone. While collecting data, I scanned 10-50 random ${ }^{3}$ MySpace profiles a day; I examined more than 10,000 teen profiles in an effort to make sense of the content that teens shared. In sampling these random profiles, my goal was to gain a general sense of typicality while also looking for the range of experiences and presentations teens portrayed. I manually saved 1,000 representative public profiles for in-depth examination.

Toward the end of my research, I began joining Facebook town networks and using those to access teens' profiles. Because random sampling was not possible, I aimed to get a broad, representative sample by selecting different types of communities and looking for a wide range of profiles. That said, my breadth was limited in part because only a small subset of teens had made their profiles visible to their towns' networks. During the process of interviewing teens, I often looked at their profiles, either with them or later.

\footnotetext{
${ }^{3}$ MySpace profiles are assigned a unique identifying number (UIN) based on the order in which they are created. Through minimal trial and error, it is possible to determine the highest UIN (i.e., the newest account created). I used a random-number generator to get a random set of UINs, which could be plugged into http://www.myspace.com/UIN to produce users' profiles. I ignored band profiles, empty profiles, deleted profiles, and profiles of people who were clearly adults. With the support of MySpace, I was able to obtain a random sample of private profiles as well as public profiles.
} 
I used profile data to examine how teens presented themselves online. During interviews with teens, I often talked about their profiles to get a sense of how they thought about their self-presentations. The combination of profile and interview data frames the foundation of my analysis of identity performance. I also use online data to ground my understanding of teen practices with respect to status and peer relations.

I did not interact with most teens whom I observed online, but I did regularly communicate with teens who approached me or who I knew from unmediated contexts. A few teens became regular commenters on my blog, providing additional information when I talked about teen practices. In 2007, I hired one of these teens, Sam Jackson (now a freshman at Yale), as an intern to help me examine online data. Together, we scoured thousands of LiveJournal, Xanga, and MySpace blog posts, looking for teens talking about their use of social media. We stored more than 200 blog posts by teens talking about their use of social network sites. Blog comments helped me access teen perspectives that I did not hear during interviews. I use this data to counter or complement the broader topology of teen practices.

\subsubsection{Interviews}

From late 2006 to mid-2007, I conducted semi-structured interviews with 94 teens from 10 states (Alabama, California, Iowa, Kansas, Massachusetts, Michigan, 
Nebraska, Pennsylvania, Texas, and Washington). ${ }^{4}$ Roughly half came from major urban regions and their surrounding suburbs (e.g., Los Angeles, Seattle), a quarter came from suburbs of mid-size cities (e.g., outside Austin, Texas, and Kansas City, Missouri), and a quarter came from smaller towns or the rural regions surrounding them (e.g., Ames, Iowa, and Lancaster, Pennsylvania). I intentionally chose diverse regions and made an effort in these regions to go to diverse communities that represented different demographics and cultural makeup.

Recruitment varied in each region. In Seattle, the library association that invited me to speak used its connections to recruit a diverse sample of teens who lived within a 50-mile radius. In Kansas, I leveraged a network of teachers through a friend who worked for the state's department of education. In Los Angeles, I worked with a youth center organizer, a teacher, and a few parents. In Texas, my second cousin connected me to a local parents' organization. In Iowa and Nebraska, I leveraged the networks of friends who grew up there and friends of friends to reach people who lived there. Extended networks of friends, parents, and teachers helped me reach teens in Alabama, Massachusetts, Michigan, and Pennsylvania. In Seattle, Kansas, Massachusetts, Iowa, and half of Los Angeles, people who knew the community helped me recruit teens from different backgrounds. I asked these adults to introduce me to a representative range of teens who reflected the diversity of the community; I explicitly asked them to avoid focusing on exceptional teens-both

\footnotetext{
${ }^{4}$ Details about teens I interviewed or from whom I quoted public online content can be found in Appendix 1.
} 
those who stood out for their achievements and those who stood out for their disobedience or failures. In doing so, I wanted to curb the tendency to emphasize extreme cases so that I could obtain a sample that included average teens. I did meet and interview exceptional teens, but they were not dominant in my sample. In other locations, I asked the networks of adults whom I spoke with to encourage teens and/or parents to contact me if they wanted to participate. I requested simple demographic information (age, gender, zip code, interests) and selected a combination of teens who I thought might show differences within a community. When I took this route, more than twice as many teens contacted me as I had time to interview. At no point did I limit my interviewees based on their technical abilities or investments.

The formal in-person interviews were semi-structured and lasted 1-4 hours, with the majority lasting between 90 minutes and 2 hours. I allowed the teens to choose where they wished to be interviewed; I interviewed teens at their houses (with parents in a different room), at schools, at coffee shops and restaurants, at youth centers, and at their places of employment. I invited teens to bring along a friend or family member of their choice if that would make them feel comfortable; roughly half of those interviewed were interviewed in pairs. In earlier projects, I had found that this sometimes helps ease the power dynamics inherent in interviews, especially when there is a discrepancy in age. I had also found that allowing pairs makes parents more comfortable, which can help with gaining access to teens. Not surprisingly, interviews with pairs tend to take longer. No doubt interviewing pairs 
alters the dynamic, but the differences are neither better nor worse. Pairs tend to correct one another or provide clarity or context to the other's answers, leading me to believe that singles are more likely to obscure or misremember the truth. When the rapport between all three of us is good, pairs are more likely to reveal embarrassing events about the other person or to push the other person to do so. Conversely, singles are more likely to indicate lack of knowledge or weaknesses. It is easier to get at - and for that matter observe-friendship dynamics in pairs while teens offer a more detailed portrait of home life when alone.

Of the teens I interviewed, 57 percent were female and 43 percent were male. The average age of the teens I interviewed was 15.9 years old. I asked teens to indicate their race and allowed them to indicate multiple races -51 percent listed white; 20 indicated they were Hispanic, Latino, Mexican, Puerto Rican, or Chicano; 15 percent marked themselves as black; 6 percent said that they had some Native American roots; 4 percent identified as Indian or Pakistani; 4 percent indicated they were Asian; and 2 percent identified as Egyptian. I did not ask for information on religious practices, but the majority of teens I interviewed referenced church during the interview; two volunteered that their families were Muslim. More than 25 percent indicated that they spoke a non-English language at home either sometimes or frequently.

While marking socioeconomic status in the United States is fraught, I attempted to interview teens who appeared to be from a wide variety of socio-economic 
backgrounds. I asked teens to indicate their parents' education, although many did not. Of those who did, approximately two-thirds indicated that they had at least one parent with a college education. I interviewed teens whose parents were lawyers and veterinarians and I interviewed teens whose parents worked the night shift at a hotel and worked at the family restaurant. Some of the teens I interviewed did not have a parent who worked and three did not live with either parent. Some teens had parents who expected them to do graduate work while others simply wanted them to stay off drugs. Not all of the teens I interviewed expected to go to college and few intended to leave their home state to do so. A handful expected to go to the military and the rest intended to work after high school. In finding teens to interview, I tried to find teens with different stories and different perspectives.

The bulk of my dissertation analysis stems from the data that I collected during interviews, set against online observations and observations of the communities in which these teens live. My analysis of peer relations and power dynamics is deeply rooted in these interviews and I use teens' words to give shape to the analysis that I do. These interviews are also what helped me map teens' online performances.

\subsubsection{Fieldwork in Less Structured Environments}

As it was not possible for me to be a true participant-observer in teen communities, I found other ways to get access to and observe teens' everyday cultural practices. I connected with numerous teens in less structured environments to gain a more general sense of what was going on and how the teens I was 
interviewing and observing online fit into a broader ecology of teen culture. These observations and informal interactions helped me ground the formal data that I collected and analyzed. I used different tactics to work around the structural limitations that I faced in getting close to teens on an everyday basis.

I spent time with kids of friends and colleagues, volunteered to help friends who were working with poor and gang youth, and sat in on classes that friends were teaching. I interviewed high-school seniors from my alma mater and worked alongside activist youth. I talked with teens at malls, on airplanes, and in churches. I never formally interviewed any of these teens, but their perspective and stories were invaluable in helping me make certain I was looking at the full picture.

In public places where teens gather, I started wearing some of the MySpace Tshirts that the company gave me. At the beginning of the MySpace phenomenon, this frequently motivated teens to approach me to ask about the shirt. When I told them that I was a researcher, they would tell me stories about how they used MySpace.

I was a guest on two teen-organized radio programs and the expert for dozens of teens' articles in their school newspapers. Teens interviewed me for their school reports and, when they wrote to get quotes, we often started conversing about what they were doing online. I moderated youth panels at conferences, helping adults hear teens' voices directly. 
Beyond youth media, I regularly served as a radio, $\mathrm{TV}$, and newspaper press expert. When I appeared, teens would write me with questions, thoughts, or requests for advice. While NPR and the New York Times provoked some teenagers to respond, I received the greatest response when I appeared on Fox's The O'Reilly Factor. One teen wrote to me on MySpace to say that even though I went to Berkeley and was probably a liberal, he really liked what I had to say and wished that I would call his mother.

At my public talks, teens were often present. Initially, these situations terrified me because I felt awkward describing teen practices with teens in the audience. Yet I found these events to be tremendously rewarding. My talks usually energized the teens in the audience and they frequently approached me to thank me, correct me, and tell me their stories. Four of my talks included large groups of teens: an online safety-planning session for teens, a countrywide religious synod, an event for teen media creators, and a conference on science that attracted high-school volunteers. After each of these talks, I was surrounded by more teens than adults and their feedback was quite helpful.

More than anything, my informal interactions with teens during the last few years helped me ground what I was seeing and develop a sense for what I might be missing. Teens would tell me where I should be looking, let me know what I was missing, and push back on my analysis. In this way, I was able to learn from and share my findings with other members of the population being studied. 
Anthropologists have argued that ethnography should involve collaboration between researchers and subjects (Lassiter 2005; Mosse 2006) and I valued the ability to share my work with those I was studying.

\subsubsection{External Data}

I have been-and continue to be-actively involved with technology companies and communities of researchers. While working on my dissertation, I was exposed to a tremendous amount of relevant data that was not part of my dissertation fieldwork. This proved to be valuable in shaping my data collection and validating my analyses. When outside data suggested findings that conflicted with my own, I was forced to work out what might explain the differences. While this did happen on occasion, prompting path correction, outside findings typically confirmed what I was seeing. By having access to outside data, I was able to focus my interviews, contextualize my mediated observations, and get a better grasp of the phenomenon. Although I do not use outside data directly in this project, I believe it is important to acknowledge that such data did shape this project.

While in graduate school, I consulted for and interned at a variety of companies involved in social media. Through these engagements, I ran focus groups, interviewed teens, interpreted data, and analyzed profile data. In return for my feedback, MySpace shared internal research with me and gave me access to some of its data. As an expert on social media, I spoke with hundreds of journalists, safety experts, youth librarians, ministers, marketers, parents, teachers, youth researchers, 
congressional staff, district attorneys, technology developers, and social workers. Many of these people shared data or anecdotes in return for my expertise. I also collaborated with various researchers at different institutions who were also collecting data about topics related to my dissertation, including the Pew Internet and American Life Project, Harvard's Berkman Center for Internet and Society, and the MacArthur Foundation-funded digital kids project at University of Southern California and University of California at Berkeley.

Throughout my project and beyond my fieldwork, I have been exposed to data about how teenagers are using technology, how adults are responding, and how society is coping. Other researchers' observations, interview data, and unpublished findings have helped inform my work and given me a sense of clarity about what I am finding. The work of colleagues has helped me contextualize what it is that I am seeing. While I do not analyze their data for my dissertation, I would be remiss not to account for how this data informed my perspective.

\subsubsection{Complexities of Online Data}

Many of the complexities of collecting data stem from the challenges I faced in setting up my field site, but I want to highlight the issues I faced in collecting online data because of privacy settings and norms. As I collected data and observed practices online, I had to ask myself two questions: (1) If content is publicly accessible on MySpace, do I have a right to access it?; and (2) When I have access to private content without people's awareness, how should I incorporate this as data? In 
essence, I struggled to negotiate the convergence of public and private contexts, an issue that I mapped out in Chapter 1 as a core complexity of networked publics.

Even when teens' content is "public," they may not understand quite how public that content really is (Stern 2004). My interviews with teens suggested that while many teens on MySpace and Facebook do not restrict adults' viewing of their profiles, they do not expect adults to view what they write. As one teen remarked, "Why would an adult want to look at my profile?" Most of the teens I interviewed did not feel as though they had something to hide, but they were dumbfounded as to why adults would visit their profiles if they did not know them. Teens expected that random strangers might stumble on their pages but presumed that the strangers would keep moving.

Just because I could view their content did not necessarily mean teens wanted me to. This is not to say that all teens objected to my viewing their profiles. On the contrary, teens frequently gave me their MySpace addresses as a way to contact them, implicitly welcoming me to view their profiles. When I told teens that I was browsing profiles, they thought it was an odd use of my time, but they were not appalled. It was clear that they had not accounted for me in their mental model of potential visitors, but they did not see me as a harmful visitor either. What worried me is that most teens had no idea that I was viewing their profiles because of my invisibility. 
Researchers have argued that, when it comes to data that is in a public/private continuum, it is important to take into consideration both content and context (Elm 2008). Most of the content that I viewed was harmless, filled with stories that were irrelevant to anyone who did not know the particular teen. Although the press tends to spin a teens-gone-wild narrative when addressing MySpace profiles, I visited more profiles with odes to Jesus than with disturbing photos. In other words, the content was simply mundane. At the same time, the context in which that content was created did not include me. Teens created their content to be consumed by their peers. Furthermore, the inequality in visibility worried me. Not only did I not fit into the context, but also my presence was nonexistent.

Given this combination of content and context, I decided to be tremendously cautious in the ways in which I captured and used public profile data. Even though the data was purportedly public, I treated it as sensitive material for the purposes of data capture and storage. I analyzed the profile content, but when using examples in my writing, I scrub all identifying information and, in some cases, alter the text so that the profile is not findable through search. To minimize potential harm, I use examples from this data set rarely and cautiously.

I decided to treat public content with such care because of the mediated nature of it. In ephemeral public situations, repeating direct quotes that are overheard is unlikely to put anyone at risk. Because of the persistent and searchable nature of networked publics, it is tremendously easy to trace a digital conversation back to its 
source. Doing so shines a spotlight on individuals, their content, and their peers. While teens' content might be public, most of it is not meant to be read by all people across all time and all space. There are cases in which teens create content that is clearly intended for a wide audiences, such as the teens who produce podcasts for Youth Radio that are then aired on NPR. Yet these cases are rare. In drawing the line, I decided to directly reference content that was clearly meant for mass consumption and to do everything possible to protect content that appeared to be intended for peers or small audiences. I decided that it was unethical to amplify content's visibility when that does not appear to be the teen's intention.

Private profiles were a different matter altogether. Although MySpace profiles were initially public-only, both MySpace and Facebook implemented privacy settings to allow users to restrict who could access their profiles. With MySpace, profiles are either public or Friends-only. Facebook's approach is more complex; teens can never make their profiles truly public, but they can make them available to everyone in their school or city. When these settings came into play, teenagers were encouraged to-and frequently did-limit access to their profiles (Lenhart and Madden 2007b). On one hand, I was glad to see teens restrict their profiles to the audience that they intended their content for. On the other, these restrictions hampered my ability to observe teen practices. Furthermore, I was concerned that if I considered only public profiles, I would get a skewed view of what was taking place. 
As it turned out, I gained access to private content in two ways: through teens and through MySpace. In interviews and conversations, teens (and their parents) regularly showed me their private profiles. Because profiles are constructed collectively, these profiles revealed just as much about those teens' friends as they did the teens themselves. Additionally, teens often showed me the private profiles of their friends and classmates and these contained comments and connections to a wider set of teens. In other words, through one teen's profile, I could see the private dynamics of a much broader swath of teens' practices. MySpace also gave me limited access to some private profiles in exchange for advice on how to approach certain situations. The company also gave me limited access to content that teens and their parents had submitted to support and abuse teams, both for advice and during my deposition in a lawsuit.

Such access was tremendously informative, but it came with ethical baggage that I was forced to confront. In both cases, I viewed content that teens produced without being its intended recipient. While public content is also not necessarily meant for me, teens had not made explicit moves to restrict who could access their profiles. Of course, private content is never truly private. Teens can copy and paste what peers write and broadcast it elsewhere on the web (a common form of bullying). Company privacy policies allow companies to use content for internal purposes and to share it with third parties under specific circumstances, including police investigations and lawsuits. Company employees access private profiles for both support purposes and research. 
Given that I believed that this material was highly sensitive and not meant for my research purposes, I decided not to document or store any information from private profiles. I observed this content and it shaped my understanding of the data that I did capture, but I did not directly analyze any of this material as part of my dissertation nor do I ever quote from such content. While access to private data required tremendous caution, I also believe that viewing both public and private content strengthened my analysis.

\subsection{Analyzing Relationships and Technology}

By constructing my field site as a network and collecting disparate data using multiple techniques in a variety of settings, I set the stage for analyzing a social phenomenon rather than a specific population or space. To analyze that phenomenon, I examine the networks of people in relation to one another, focusing on the role that technology and other mediating objects play in those networks. My analytic approach focuses on three different sets of relationships: (1) the relationship teens have to themselves as they engage with networked publics; (2) the relationship teens have to their peers through networked publics; and (3) the relationship that teens have to parents and other adults who hold power over them as a result of networked publics. My dissertation is organized around these analytic categories and a chapter is dedicated to each of these three. I use a combination of cultural and structural analysis and draw on different types of data to determine the dynamics involved. 
In the first section of my dissertation, I analyze how teens negotiate selfrepresentation and impression management in social network sites. My approach is primarily a cultural analysis in which I interrogate how social network sites alter everyday practices involving identity and context. I use data collected through MySpace profiles and interview data concerning the decisions that teens made as they crafted their profiles. My analysis works to understand the relationship between what teens view to be their practices and what the traces of those practices signal.

The second section of my dissertation explores how teens relate to their friends and peers through and because of networked publics. My analytic approach in this chapter is primarily structural in that I examine how technology replicates, reinforces, and alters the practices of peer relations. I bridge interview data about teens' practices with their friends and peers with an analysis of the properties and features of social network sites. The interview data that I use includes discussions of unmediated peer relations as well as those involving networked publics. My analysis focuses on how networked publics alter the structural conditions for peer management and the ways that teens negotiate this.

In the third section of my dissertation, I examine the relationships between teens and adults in relation to the social network site phenomena. The bulk of this chapter centers on the moral panics that emerged and the ways in which teens and adults navigated these fears and their relationship to one another. My analysis primarily takes a structural form and focuses on the power that adults have in 
controlling teens' relationship to public spaces, including networked publics, and teens' efforts for agency in this context. While the bulk of this chapter focuses on the struggles that teens and adults face in relating to one another, I also spend time analyzing more positive interactions between teens and adults as a result of these sites. I draw primarily on interview data concerning teens' relationships to their parents and other adults, but I also use teen blog posts, mass media news coverage, and discussions with adults.

My field site, data collection, and analysis rely heavily on networks-networks between peoples, between spaces, and between practices. The analytic process that I take in working through my data is centered on these networks and includes both cultural and structural approaches. My emphasis is on the relationships and the movement, not on what is static. While I was not able to collect data in a continuous fashion across these networks, I attempt to bridge the gaps analytically by moving between different bodies of data, constantly trying to evaluate each set of data from different angles and to understand contextual information that is both accessible and inaccessible. 


\section{Chapter 3: Social Network Sites and Social Media}

Social network sites like MySpace and Facebook are a relatively new genre of social media and a part of a long lineage of networked publics. The term "social media," as I am using it, is an umbrella term that refers to the set of tools, services, and applications that allow people to interact with others using network technologies. Social media encompasses groupware, online communities, peer-topeer and media-sharing technologies, and networked gaming. Instant messaging, blogging, microblogging, forums, email, virtual worlds, texting, and social network sites are all genres of social media. Social media is sometimes referred to as "social software" or "social computing" or "computer-mediated communication." Most gen res of social media leverage personal computers and the Internet, but increasingly, mobile networks are serving as an additional key network technology. Social media includes systems that support one-to-one, one-to-many, and many-tomany interactions. Some enable many-to-many interactions and support the creation of spaces for people to gather and publics to form. I call these spaces, and the resultant collective, "networked publics." Usenet, the blogosphere, and social network sites are all examples of networked publics.

The power of social media is rooted in its ability to connect people across time and space. The way these tools are used alters a plethora of practices, including communication, collaboration, information dissemination, and social organization (Benkler 2006; Castells 1996; Rheingold 2002). Computer code does not determine 
practice, but as a form of architecture (Lessig 2006), it does shape the way in which people can interact. Social media has affected how people interact with one another and, thus, it has the potential to alter how society is organized. Although they are simply the messengers, social media tools are revered for their potential to connect (Shirky 2008; Tapscott and Williams 2006; Weinberger 2008) and despised as corrupting forces (Keen 2007).

The history of the Internet is entwined with social media. Social media is as old, if not older, than the Internet. A local instantiation of email predates the moment when, in October 1969, machines at Stanford and UCLA were able to first talk to one another through the nascent ARPANET, which would later become the Internet. ARPANET popularized email and email became the "killer app" for ARPANET, especially after Ray Tomlinson initiated the use of the @ sign to uniquely identify users on individual machines. ${ }^{5}$ Networked publics followed almost immediately. The first public bulletin board system (CBBS) and the first multiuser dungeon/domain (Essex MUD) were both created in 1978 and Usenet was launched in $1979 .^{6}$ Chatrooms, instant messaging, and mailing lists all have roots that extend to the 1970s. Different instantiations of these services took root in the 1980s alongside the emergence of "virtual communities" (e.g., the WELL). After the 1993 launch of

\footnotetext{
${ }^{5}$ Much of this is well documented and referenced on Wikipedia in the entries on "Email" and "History_of_the_Internet."

${ }^{6}$ These early networked publics are well documented at Wikipedia in the entries on “Computerized_Bulletin_Board_System," "MUD," and "Usenet."
} 
Mosiac popularized the World Wide Web, a whole new set of social media tools began to form. Online dating sites, blogging, social bookmarking, and wikis are just a few examples of web-native genres.

Social network sites are but recent developments in the history of social media. They are their own genre, but they also build on prior forms of social media. Their uniqueness lies in the combination of their features and the structures that allow people to demarcate relationships. In trying to locate this uniqueness, Nicole Ellison and I defined "social network sites" as "web-based services that allow individuals to (1) construct a public or semi-public profile within a bounded system, (2) articulate a list of other users with whom they share a connection, and (3) view and traverse their list of connections and those made by others within the system" (boyd and Ellison 2007). The profiles of most social network sites resemble those of online dating sites, in part because the early social network sites were designed to be online dating sites (boyd and Ellison 2007). The articulation of relationships resembles that which takes place through IM and address books, but the public formalization of this is unique.

Social network sites incorporate features from a wide array of other genres of social media, including blogs, instant messaging, email, bulletin boards, chatrooms, and media-sharing sites. They are an amalgamation of many prior genres. In choosing to label them "social network sites" instead of the more common nomenclature "social networking sites," Ellison and I sought to emphasize that what 
makes them unique is the way in which they allow people to articulate relations, not the ways in which they allow people to meet new people online. We thought the latter emphasized the wrong things and, in doing so, it expanded the scope to reference a much wider subset of social media tools.

Two social network sites-MySpace and Facebook-play a central role in my dissertation, both as elements of my field site and key case studies for my analysis of networked publics. These sites are not the first social network sites, but they are the most relevant to my study. To help the reader understand the practices being discussed, I want to locate these sites in a broader history of social network sites, document the dynamics that surrounded the emergence of these sites as a phenomenon among youth, and place teens' engagement with social network sites into a broader context of teen social media adoption. For those wholly unfamiliar with MySpace and Facebook, I have also detailed their features and functionality in Appendix 2.

The history of social network sites that is mapped out here is an abbreviated version of that which is outlined in boyd and Ellison (2007); the data for this history comes from interviews I conducted between 2003 and 2007 and field notes that I took during that period while studying this phenomena. In locating the phenomenon and discussing broader teen social media practices, I combine prior literature, news media, and explanations offered by teens I interviewed. This chapter is not intended as an exhaustive review of social media or teen practices, but instead, 
it serves to provide critical context for those wanting a better understanding of how the sites that I used in observing teen practices fit into a larger picture.

\subsection{The Rise of Social Network Sites}

Social network sites grew out of prior forms of social media. Because many of the features existed in previous forms of social media, it is challenging to locate the origin of social network sites. Arguably, using the definition Nicole Ellison and I devised (boyd and Ellison 2007), the first recognizable social network site was SixDegrees.com, launched in 1997. SixDegrees allowed users to create profiles, list their Friends, and, beginning in 1998, surf Friends lists. While profiles and buddy lists existed before SixDegrees, SixDegrees was the first to bring them together in a recognizable form. An earlier site, Classmates.com, allowed people to affiliate with their high school or college and surf the network for others who were affiliated, but users could not create profiles or list Friends until later. SixDegrees was the first to truly integrate the different features that constituted a social network site.

SixDegrees eventually shut down; its founder believed that it was ahead of its time (Andrew Wein reich, personal communication, July 11, 2007).

Throughout the late 1990s, other online communities and social media tools began implementing social network site features, arguably turning their online communities into social network sites. LiveJournal, Asian Avenue, and Cyworld are just a few examples of online communities that launched during this period and eventually became social network sites through the addition of key features. While 
social network site infrastructure was proliferating, profile-driven social network sites like SixDegrees reemerged when Ryze.com and Friendster were launched in 2001 and 2002, respectively.

Ryze was designed as a business-networking tool and Friendster was intended to improve on the popular online dating site Match.com by adding information about people through their social networks. Ryze achieved limited success, but Friendster gained tremendous popularity in 2003, primarily by word of mouth (O'Shea 2003). Friendster's popularity set in motion a wave of development in this area. Friendster should have dominated the market, but what it gained by being the first to attain reasonable success, it lost through a series of technical and social missteps, most notably the company's determination to configure how its early adopters engaged with the site (boyd 2006a, 2008b).

Designed as an online dating site (Cohen 2003), Friendster wanted users to provide accurate information, connect only to people they knew and trusted, write formal testimonials on each other's profiles, and engage with others in preconstructed ways. The site's users had much more creative ideas about how the site should be used. Some treated profiles as artwork and creatively represented themselves, groups, celebrities, institutions, objects, and ideas through these profiles (boyd 2008b). The logic behind which people connected was varied and the public display of those connections shaped people's approach (Donath and boyd 2004). Participants began collecting Friends and competing to be most popular as 
measured by Friend count (boyd 2008b). Testimonials were repurposed as public spaces for conversations and participants developed a wide array of practices that had nothing to do with online dating. The company was not pleased. While participants were "socially constructing" the system (Bijker et al. 1987), the company turned around and tried to "configure the users" (Grint and Woolgar 1997). This did not work and many users rebelled. Some turned their attention elsewhere; others lost interest.

MySpace was able to capitalize on Friendster's alienation of many of its early adopters, especially when users began encouraging their Friends to switch services after a subscription fee scare (Tom Anderson, personal communication, August 2, 2007). MySpace wanted to attract estranged Friendster users (Tom Anderson, personal communication, February 2, 2006); it was designed to be a close replica of Friendster with additional features that resembled other popular social media sites and fewer limitations (Tom Anderson, personal communication, August 2, 2007). Although the founders of MySpace realized that social network sites could be used for more than dating, they included all of the dating features. Many frustrated Friendster users left Friendster and joined MySpace. One of the notable groups that transferred allegiance included musicians and indie rock bands. They had joined Friendster to connect to fans, but Friendster had deleted many of their profiles, claiming that they were not legitimate users. MySpace, on the other hand, supported their practices. 
Like Friendster, MySpace initially attracted 20-30-something urbanites. Many of MySpace's early adopters were passionate about music, primarily indie rock music and hip-hop. While Friendster had forbidden bands from creating profiles, MySpace encouraged bands to use the site to communicate with fans (Tom Anderson, personal communication, September 28, 2006). MySpace even developed features to further support the bands, an act that was greatly appreciated. In Los Angeles, promoters began leveraging MySpace to promote shows by offering VIP passes to those who Friended them. Fans valued this practice and encouraged their friends to join to take advantage of the opportunity.

\subsubsection{MySpace and Teens}

Although individual teenagers joined MySpace early on, teens became a visible demographic on the site in 2004. Unlike the 20-something crowd, few teenagers on MySpace came from Friendster. Most early adopter teens were attracted to MySpace through one of two paths: bands or older family members. Teens who learned of MySpace through bands primarily followed indie rock music or hip-hop, the two gen res most popular on MySpace early on. While many teens love music, they are often unable to see their favorite bands play live because bands typically play in $21+$ venues. MySpace allowed these teens to connect with and follow their favorite bands. Early adopter teens who were not into music primarily learned about the site from a revered older sibling or cousin who was active in late-night culture. These teens viewed MySpace as cool because they respected these family members. As with 
older users, the first wave of teens to inhabit MySpace primarily lived in California, but teens in other states quickly followed.

Teenagers who joined MySpace began proselytizing the site to their friends. Given its popularity among musicians and late-night socialites, joining MySpace became a form of subcultural capital. Teens, especially those in urban settings, tend to look to the 20-30-something crowd for practices that they can emulate. Drinking, for example, is sometimes viewed as "cool" because it is an act that marks adulthood. MySpace's early popularity among teens was tightly entwined with its symbolic reference to maturity, status, and freedom in the manner espoused by urban late-night culture. While teens often revere the risky practices of this older cohort, many adults work to actively dissuade them from valuing these acts. By propagating and glorifying 20 -something urban cultural practices and values, MySpace managed to alienate parents early on.

With little mass media coverage of MySpace before News Corporation acquired the company in mid-2005, many teens learned of the site through word-of-mouth networks. Teens learned about MySpace from friends at school, church, activities, and summer camp, as well as from older family members. West Coast teens learned about it before East Coast teens, and urban teens joined before suburban or rural teens. While teens began flocking to the site in 2004, their participation was significant by mid-2005 and the media coverage that followed the sale further popularized the site. 
Immediately after the sale of MySpace to News Corporation, much of the media coverage focused on the bands. After adults began realizing how popular MySpace was with teens, the media became obsessed with teen participation and the potential dangers they faced. This media coverage was both a blessing and a curse for MySpace. On one hand, many teens joined the site because media sold it as both fashionable among teens and despised by parents. On the other hand, many teens avoided joining because of the perceived risks.

As I began my fieldwork, safety concerns emerged as a dominant theme that shaped teens' perceptions of MySpace. Some teens worried about their safety, particularly with respect to pedophiles. Jennifer, a white 17-year-old from Kansas, did not want to join MySpace because "pedophiles and stufflike that kind of scared me away." In some places, individual teen fears turned into school wide stigmas concerning the site. Laura, a white 17-year-old with Native American roots from suburban Washington, told me that everyone at her school "looks down on MySpace because they think everyone is putting themselves in danger." Elsewhere, teens rejected these fears as irrational adult concerns. How teens negotiated adult concerns is a central theme of Chapter 6.

As MySpace grew and simultaneously appealed to and scared off U.S. teens, other social network sites started gaining traction with different demographics. Most did not appeal to teenagers en masse, although niche groups of teens did join a wide array of different sites. In particular, teens from immigrant families who still 
had strong connections to family and friends outside of the United States often joined sites that appealed to those geographic communities. This phenomenon also came out during my interviews. For example, Korean 17-year-old Seong used Cyworld to stay connected with her friends and cousins back home. She was born in Korea but moved to Los Angeles when she was young and she loved having a tool to reconnect with friends and family. On the other hand, Anindita, an Indian 17-yearold girl from Los Angeles, joined Orkut upon the insistence of her Indian cousins, but she found this site too confusing to fully engage.

\subsubsection{Facebook and Teens}

While teenagers were adopting MySpace en masse, Facebook began gaining traction with college students. Facebook started as a Harvard-only social network site before expanding to support all Ivy League schools and then top-tier colleges and then mainstream colleges. Initially, only college students from preapproved schools could join Facebook and they had to prove their collegiate affiliation by signing up with their .edu email addresses. The college-centered nature of Facebook quickly appealed to teenagers who saw access as a rite of passage. They were aware of the site through older family members and friends from high school who had already graduated and gone off to college. Before access became readily available, collegebound teens began coveting access. In spring 2005, I spoke with college computingsystems administrators who were surprised to receive messages from high school seniors seeking access to their .edu email addresses shortly after being accepted as 
upcoming college freshmen. These students explicitly wanted those .edu email addresses to get access to Facebook. For many college-bound teens, access to the social world of college students became a status marker.

Originally, access to Facebook was intentionally limited. This, combined with its origins at Harvard and the way the company initially opened up only to other Ivy League colleges, marked Facebook as an "elite" social network site. The highbrow aura of Facebook appealed to some potential participants while repelling others. Those bound for college saw access to Facebook as a rite of passage, a sign that they were no longer high-school students but instead college students. Even those who had MySpace accounts relished the opportunity to gain access to the college-only Facebook as a marker of transition. In September 2005, Facebook began slowly supporting high schools as networks, allowing teenagers to join the site. While this gave some teens access, the processes in place for teens to join and be validated as high-school students were challenging, creating a barrier to entry for many potential participants. Those who managed to join were quite motivated, typically because they had close friends in college and wanted an easy way to communicate with them. A year after allowing high-school students to join, Facebook opened access to anyone. This sparked a wave of teen adoption.

While many teens valued the opportunity to join Facebook, college students were not thrilled to have high-school "kids" on what they perceived to be their site. In the Daily Princetonian, Danny Shea and Matt Feinstein (Shea and Feinstein 
2006) wrote an open letter to Mark Zuckerberg, the founder of Facebook, bemoaning the addition of high school students:

"But last week, when we each accepted friendships from girls born after the fall of the Berlin Wall, we got angry. Really angry. Suddenly, we had to begin removing tags from photos of us drinking, erasing wall postings referring to awkward hookups and getting rid of anything else that might negatively influence younger siblings or get back to once-adoring high school teachers. But even beyond that, there's just something about high school facebook that feels wrong. It is annoying. It is embarrassing."

College students lamented teen access, but this did not stop massive teen adoption. Teens were not the only non-college student population to join once Facebook opened access. As I write this, Facebook has achieved tremendous popularity among a wide array of different populations of all ages and nationalities.

The segment of teens that initially flocked to Facebook was quite different from those who were early adopters of MySpace. In both cases, the older early adopters shaped teen engagement. With MySpace, 20-something urbanites and musicians set the stage for teen adoption. College students-and particularly those from top-tier universities-played a more significant role in shaping teen adoption of Facebook. In Chapter 5, I discuss the divisions around these sites in greater depth. Teen engagement with each was shaped by both the technology and the social context in which these sites emerged. 


\subsubsection{By the Numbers and in Practice}

Shortly after launching in 2003 and 2004, respectively, MySpace and Facebook became significant phenomena in the United States. Although they attracted different populations, teens quickly became a significant demographic. There is little quantitative data on the size of the phenomenon or the makeup of participants and nonparticipants. In late 2006, the Pew Internet and American Life Project found that, of the 93 percent of U.S. teens ages 12-17 it surveyed who had Internet access, 55 percent reported having a social network site profile when asked in front of their parents (Lenhart and Madden 2007a). Using survey data collected throughout 2007, Forrester Research found that 80 percent of online teens ages 12-17 visited social network sites and half of online teens visited at least weekly (Li 2008). Since then, analysts have reported continuous growth (Bausch 2007; Lipsman 2007, 2008). In 2006 data, Pew wrote that 85 percent of teens who reported having profiles were using MySpace while only 7 percent were on Facebook (Lenhart and Madden 2007a). This has undoubtedly changed, but there is no public data on the split.

Although I never met a teen who had not heard of either MySpace or Facebook, I met countless teens who had opted out. I detail some of the reasons that they chose not to participate in Chapter 5.

Teens learned about and joined social network sites to connect with their friends and peers. While networked publics have a long history of being used to help strangers connect around interests, teens' adoption of social network sites was 
driven primarily by their desire to connect with people they already knew. Pew found that 91 percent of the teens they interviewed in 2006 who use social network sites do so to stay in touch with friends they see every day, while 82 percent indicated they used the sites to connect with those they see on a less regular basis (Lenhart and Madden 2007a). They also found that 49 percent of teens use the site to make friends (Lenhart and Madden 2007a). In my research, I followed up on this latter practice and found that there are many who do make new friends through social network sites, but they do not do so in the way that most people imagine. Rather than connecting with complete strangers, these teens use social network sites to get to know friends-of-friends and build connections with classmates they do not know. It is not clear how many of those who use social network sites to make friends do so to connect with complete strangers.

The friend- and peer-centric nature of MySpace and Facebook use is significant. This is a shift away from the dominant interest-driven paradigm in networked publics. Most of the earlier popular genres of networked publics—Usenet, mailing lists, chatrooms, and so on-are organized around topics. People who used these spaces often gathered with strangers who shared their interests. While other gen res of social media have been primarily friendship-driven and while some people have always used networked publics to gather with people they knew, social network sites are almost exclusively organized around friends, not topics or interests. 
Social network sites are also the first form of social media that the majority of teens have adopted to engage with peers in a semipublic way. While blogs enabled similar dynamics, only 28 percent of online teens have ever created one (Lenhart et al. 2007). Other forms of social media that garnered widespread adoption among youth-email, instant messaging, and text messaging-were predominantly one-toone or small-group oriented. Social network sites allow for large-scale interactions but are being adopted primarily to enhance preexisting networks. Regardless of the sites' potential, teens primarily use social network sites in ways that reinforce and replicate unmediated social dynamics. Teens are not engaging with social network sites as a separate or distinct world but as an extension of everyday life. Not only do social media extend the individual (as per Marshall McLuhan [1964]), but social network sites also appear to extend the social context and peer culture.

\subsection{Participation in Context}

As teens adopted social network sites, they did not necessarily stop using other forms of social media. Pew found that teens use many different and complementary forms of social media to communicate and share information with friends and peers (Lenhart et al. 2007). The landline telephone is still extremely valuable to teens, although the rise of cell phone plans with unlimited calling options cut into landline use. Email use is declining, but instant messaging and text messaging are still widespread. Blogging has not reached widespread adoption, but some teens still blog as a way of connecting with friends. To contextualize teens' use of social network 
sites, I asked teens about their communication choices more broadly. What I learned reflects the numbers that Pew is finding, but the qualitative data helps flesh out the logic behind how teens use different forms of social media and how they see social network sites' fitting into that media ecology.

In asking teens why they started to use specific gen res of social media, I heard a common refrain: "That's where my friends are." Teens adopt the tools that their friends use and, in doing so, they influence those around them. As discussed in Chapter 5 with respect to MySpace and Facebook, the influence of peers creates network effects. This explanation holds for almost all types of social media.

Using the right technologies is important for staying connected and keeping up. For example, white 15-year-old Myra from Iowa acquired an AIM account after she started feeling out of the loop. She explains:

"I felt really disconnected from a lot of my friends 'cause they would always [ask], 'Oh, were you talking to this person? And did you find out about this kind of thing going on?' I'm like, 'No. What's happening?' So that really motivated me to and I thought it would just be easier to stay connected and stuff."

Myra joined AIM because her friends had and she continues to use it because her friends do. At the same time, when collectives stop using a gen re of social media, there is a ripple effect. In Los Angeles, Ana-Garcia, a Guatemalan-Pakistani 15-yearold, stopped using Xanga after her friends did. She explained, "If I went on there, there would be no comments or-and everyone says that's why they stopped, so no 
one does it anymore." From Ana-Garcia's point of view, Xanga's popularity ceased instantaneously. There was a time when her entire peer group was engaged and then "just everyone stopped."

Network effects drive social media adoption because collective action is necessary for a genre to gain traction and it can equally un ravel participation. In some ways, these patterns appear fadlike, as certain gen res are viewed as "cool” and then "passé." Yet, unlike fashions that go through fads, participation in social media is directly tied to others' participation. There is no value in being on AIM, cool or not, if no one else is because participation relies on others to be engaged. Furthermore, unlike fashion, social media is most effective when there is high network density. In other words, genres of social media are most valuable when everyone is engaged. That said, there are exceptions and social media does decline in status when it spreads far enough that teens are forced to navigate social situations with people they do not want to interact with, namely those they do not like, those who hold power over them, and those who have malicious intentions. The ideal is high network density among those they like and low network density among those they do not. While specific gen res of social media ebb and flow, many teens are engaged with multiple genres at one time. They have multiple channels through which they can communicate with those around them. 


\subsubsection{Negotiating Multiple Communication Channels}

In asking teens about which channels they use and how they decide among them, I found that teens recognize that each genre has its strengths and weaknesses. As other scholars have noted (Bryant et al. 2006; Grinter and Eldridge 2001; Grinter et al. 2006), teens use different communication channels in different interactions. In choosing which channel to use, teens account for the social situation, the technical affordances, the people they are trying to reach, and their own preferences. For most of them, multiple genres of social media are embedded into their lives and they leverage whichever one they think makes the most sense given a particular encounter.

For example, Kat, a white 15-year-old from Massachusetts, mapped out the ways in which the channel was dependent on the purpose of the interaction and her expectations of how her peers consumed these different media. As she explains,

"It depends on why I want to contact them. If I'm calling Jessica to see if she can give me a ride to dance, it's always phone. If I have a question about homework, I'll do it on IM, because Facebook is really riskier, because you don't know when they'll answer you, or you don't know if they're signed on, but they're not at their computer at all. So I usually use IM if they're online and I have a question. I don't use email for my friends. I use it for usually like adults."

Kat does not universally prefer one channel to another but sees each as valuable in different situations. 
Timing is a key issue that many teens bring up. Teens account for the affordances of the technology with respect to timing and take into consideration the usage and lifestyle patterns of their friends in choosing how to best contact them. As Laura, the 17-year-old from Washington, explains, "In urgency, I use texting. If it can wait, I use MySpace." She takes into consideration the priority of a message, but she also accounts for the weight of it in relation to the cost of the contact. For many short messages, she prefers texting or MySpace "because sometimes you only need to say one little thing that doesn't require a whole phone call." Laura, like many of her peers, reserves phone calls for conversations and prefers to avoid them when the goal is to communicate something simple. Another timing-related issue that teens account for is the disruption factor. While instant messaging and texting are often framed as interruption technologies, the teens I interviewed often saw them as less invasive than voice-based channels.

With text messaging and mobile phone calls, economic issues are also at stake. Text messaging is often a desirable medium for one-to-one communication, but the cost can limit use. For example, white 15-year-old Catalina from Austin, Texas, chooses to send texts only when she is out because at home, "you're not gonna waste the text." The teens I interviewed are often familiar with the costs of phone plans and they often know which friends have what carriers, what phone and texting plans, and which limitations. Teens are conscious of how their acts affect their friends. Some check to see if a friend is on IM before sending him or her a text message. Many ask their friends about their phone plans, determining whether or 
not they share the same carrier and how many texts they can receive. In Michigan, white 16-year-old Bianca is required by her father to ask her friends about their plans before she can communicate with them. Others avoid text messaging because of the potential economic impact of communication. Those I interviewed who switched from limited to "all you can eat" text-messaging plans started texting much, much more. Because most teens do not have control over their phone plans, parents often limit their phone options.

While there is gen erally a sense that high bandwidth channels are "better," teens believe that the technical affordances of social media can provide a valuable safety net in some situations. In Nebraska, white 15-year-old Brooke prefers to use AIM to talk with friends when she is hurt or angry. She explains that she is shy and that AIM allows her to compose her thoughts and communicate her feelings without being silenced. At the same time, she notes that she has come to believe that talking is "better" and she is trying to learn to vocalize her feelings. For Ty, a black 17-yearold in Los Angeles, flirting through social media is far less intimidating than doing so offline. Furthermore, there is less cost. As Ty puts it, "If they don't respond or they don't say the things I want to hear, then I'll just go on to somebody else. It's as simple as that." For other teens, social media lets them be more cautious. Some teens spend a long time crafting a message to send to a crush, providing relief from the awkwardness of approaching someone in person and risking stumbling over words. Melanie, a white 15-year-old in Kansas, points out that the "computer mask" is extremely valuable for learning how to negotiate relationships, especially in middle 
school. As she explains, "It's so much easier to say what you're thinking when you're not looking at someone." Thus, while higher bandwidth channels are still generally preferred, social media and low bandwidth channels can be especially valuable when learning how to navigate awkward or new situations.

The rise of social network sites did not introduce just one new communication channel, but a plethora of them. The affordances of the three most popular-private messages, comments, and bulletins-are each significant. As teens adopted social network sites, they treated each channel differently. Private messages are structurally similar to email, but teens can count on their friends' checking these more often and they do not have to remember their friends' current email addresses. Comments are more personal than bulletins and yet more public. Comments are often valued for lightweight conversations when it is acceptable for the interaction to be "overheard." Furthermore, because of the public nature of comments, comments are often used as a tool for marking and negotiating status, forcing teens to carefully craft their posts at times. In choosing between private messages and comments, teens often consider the intensity of the connection. For example, Jill, an El Salvadoran 14-year-old from Los Angeles, explains that she thinks "a message is like if you want to like to a person like talk and talk and the comment is just like to just drop by and say 'how are you' and stuff." MySpace bulletins provide a one-to-many channel where teens can broadcast their thoughts for everyone they know to read. This is effective when people send messages infrequently, but collective abuse can kill this channel and many of the teens I interviewed complained about getting too 
many updates to bother reading their bulletins. Bands often use this channel to mass market and it became a preferred channel for spammers who phish accounts to send mass marketing messages to potential customers. Yet continual chain mail, quizzes, and requests for comments can also start to look like spam, even when they come from friends. Other lightweight channels of communication-like Facebook's "poke" feature-are nothing more than a tool to acknowledge someone's presence.

In choosing which channel to use for a particular interaction, teens balance many different factors. Mentally, they associate different channels with different types of content, for use in different situations, and for specific people. This results in a segmentation of people and content into different buckets based on tool. For example, a teen might view email in relation to teachers and homework, MySpace as the tool for joking with classmates and flirting, and IM as the channel for intimate conversations with friends. Not all channels are created equal and negotiating social interactions requires choosing which channels are best for which social situation. Teens have many choices, but this process cannot set by an individual; it must be negotiated collectively.

\subsubsection{Teen vs. Adult Social Media Practices}

Through the years, teenagers have adopted a wide variety of social media and communication tools. When it comes to genres of social media for communication, teen use often precedes broad adult adoption. Teens played an important role in the popularization of instant messaging, beepers, mobile phones, text messaging, online 
journals/blogs, and social network sites. They were by no means the only early adopters, but they were significant adopters and often shaped how the tools evolved.

Teens' use of social media is primarily visible when teens develop or adopt practices within these genres that appear distinct from adult practices. Usually, practices common among teens raise concerns among adults who do not adopt the same patterns. An example of this is the use of MiXeD cAsE tYpInG or txting $n$ im msgs w/ wassup, lol, ttyl in em. ${ }^{7}$ While teens often view these practices as expressive or valuable shorthand, scholars raise concerns that these semi-oral, semi-written practices may affect or "damage" written communication and language (Baron 2008).

Practices that are particularly common among teens are also often ridiculed, even when adopted by adults. For example, the dominant use of blogging software—by both teens and adults—is to share personal content among friends in a style that resembles journaling or keeping a diary. Another style of blogging resembles amateur journalism, but only a fraction of the blogosphere comprises content written for massive consumption in the style of amateur journalism, political commentary, or tabloid exposé. Yet it is this approach that is typically viewed as a more productive use of these tools. Services that support the blogosphere make this explicitly clear by offering lists of the "best" or "top" blogs based on quantity of links or size of readership. There are no such lists for depth of

\footnotetext{
${ }^{7}$ In standard English: An example of this is the use of mixed-case typing or texting and instant messaging shorthands that effectively mean "what's up?", "laughing out loud", "talk to you later."
} 
engagement. Whenever mass media wants to demean bloggers, they are labeled as "web diarists" and condescending references to teen practices are typically employed. For example, in 2004, the New York Times ran an article titled "Web Diarists Are Now Official Members of Convention Press Corps" as a not-so-subtle critique of the Democratic Party's willingness to include bloggers as journalists (Lee 2004). The photograph that the newspaper ran with this story was of a 16-year-old blogger.

While teens may adopt the same tools as adults, they often use them for different purposes or in different ways. For example, email is the all-purpose communication tool for most adults while it is used by teens for a much narrower set of purposes. Teenagers rarely use email to socialize with friends. It is primarily a tool for communicating with parents, teachers, and other adults or to sign up for websites or commercial offers. As a social tool, email is passé. In explaining why he preferred using MySpace over email when talking to peers, white 17-year-old Michael from Seattle noted that "email's boring-There's no pictures, there's no 'about me.' There's no colorful background." Kira, a Latina-white 17-year-old also from Seattle, took Michael's complaint one step further by noting that MySpace is easier "because then you just click on their picture, you can either leave them a comment, a message, whatever." She also noted that many of her friends' emails bounce, making it frustrating to try to contact friends this way. Teachers, on the other hand, expect teens to contact them via email and their emails do not bounce. As teens lamented email and indicated why they preferred social network sites or 
other forms of social media, they highlighted technical affordances that have made this channel less appealing. Most of the value of email can be replaced with other genres. The only exception is sending files and many teens still use email to send files to their friends. Although many teens have email addresses, they are quite different from their parents in their lack of attachment to them.

Similar patterns are playing out with social network sites. Teens were among the earliest of adopters, but adults have since followed. Yet what teens do on social network sites appears different from what adults do. Little data exists on exactly how adults are using social network sites, but in my viewing of both teen and adult profiles during the last few years, it is clear that the traces of their use are quite distinct. Teens appear to spend more time there, hanging out and socializing with people they know, changing their profiles, and leaving comments. Adults, on the other hand, appear to be more likely to "network," using these technologies to reconnect with old acquaintances, find business connections, or date. They are less likely to write comments regularly, update their profiles, or add photographs. The differences in behavior do not stem from the technology but are most likely driven by how these tools fit into different groups' everyday practices.

Adults and teens also have different reactions to and coping mechanisms for handling similar dynamics. For example, teens found Friending to be awkward because it complicated their relationships with their peers while adults stumbled over having to manage professional acquaintances and long-forgotten classmates. As 
discussed in Chapter 5, many teens developed strategies for navigating these complicated social dynamics, but journalistic stories continue to highlight the difficulties adults are having with this aspect of social network sites (Jerigan 2008; Swidey 2008). The differences may stem from the ways in which teens learn to manage relationships during the period in which they learn to negotiate social network sites, while these sites are forcing adults to develop new skills to handle new social situations.

Although both teens and adults may adopt many of the same technologies, it is important to examine how teens engage with social media separately from how adults use these tools because teens' participation is not simply an extension of adult practices. As teens began engaging with social network sites, they developed interaction models that were quite distinct from the ways in which adults were previously using these sites. Likewise, the ways in which these tools shape their lives are different and teens come to these sites with different expectations and presuppositions than adults. The next three chapters highlight some of core teen practices, showing how teens' practices involving these sites are connected to broader dynamics that structure their lives. 


\section{Chapter 4: Writing Oneself into Being}

“i'm allie. i'm not a very complex person.

i like music. i like reading. i love meeting new people.

i'm still trying to figure a lot of things out. about God. about life. about my future. about people.

i don't like who i am, but i'm working towards being someone better.

i'm really trying to follow Jesus with my all. sometimes i need help."

-Allie, a white 17-year-old from Indiana, in her "About Me" on MySpace

Writing a biographical statement can be challenging. The ways to describe oneself are countless and choosing which way is appropriate wholly depends on the context. Yet even with the context in mind, successfully portraying oneself is not simple, whether on paper or in person. In an effort to make a good impression, people tend to look around, see how others are acting in that context, and choose their performance accordingly. Depending on how they are received, people alter their behavior to increase the likelihood of being perceived as intended. Such is the essence of what Erving Goffman (1959) calls "impression management," including the processes involved in the "presentation of self."

Mediated environments like networked publics formalize and alter the identity processes of self-presentation and impression management. Teens must formally make their presence known through the explicit creation of profiles, and the 
iterative acts of impression management are complicated by the limited social feedback in online environments. More challengingly, teens must do so in an environment whose context is unclear and constantly changing.

Allie’s “About Me" self-description on her MySpace profile reveals her attempt to textually locate herself. Alongside this description, Allie offers a grinning selfportrait, an eclectic list of interests, an assortment of media tastes, simple demographic information, reflective blog posts, and a melancholic song to flesh out her digital self-representation. Her list of Friends and the comments they leave provide insight into her social world, reflecting on her identity. While Allie's MySpace profile is filled with information about who she is, the very creation of this profile is a social oddity, in the sense that hers is the first generation to have to publicly articulate itself, to have to write itself into being as a precondition of social participation.

As rich as her profile is, it is hard to place Allie. Her self-description reveals some angst, but she is glowing in her photograph. Her self-description has stayed the same for months, making it difficult for me to sense change. I can gather from her five blog posts over two years that she is struggling to make sense of her religion, but I have no idea how pervasive these thoughts are in her daily life nor can I tell why this is the only topic that she seems to post about. I can see whom she lists as Friends, but I have no idea how she feels about these people or those she spends time with who are not on MySpace. Her profile is public, which makes it possible for me to see, but 
I have only the slightest idea of whom she intends to view it. For all that is revealed, there is much more that is not.

Every day, in unmediated environments, people ritually seek to manage the impressions they make in social interactions. This impression management requires them to negotiate, express, and adjust the signals that they explicitly give and those that they implicitly give off. In his seminal text, Goffman (1959) details the ways in which people take into account the social situation and their role in it to use body language, speech, and other people to convey an impression. What Goffman's account does not foresee is the way that mediated situations might alter this process.

Much of what people take for granted in unmediated situations cannot be accounted for in mediated ones. Online, there are no bodies in the corporeal sense, obscuring both identity information that is typically written on the body and presence information that makes a person visible to others. To exist in mediated contexts, people must engage in explicit acts to write themselves into being. On social network sites, this means creating a profile and fleshing out the fields as an act of self-presentation.

While creating a tangible digital identity is relatively simple, negotiating the technology to engage in acts of self-presentation and impression management is complex and different from how these acts play out in unmediated environments. The processes of social signaling are complicated by technology, altering how teens can gain access to impression-management fundamentals: context, explicit 
feedback, and implicit reactions. The persistent, searchable, alterable, and networked nature of these environments makes it difficult for teens to locate their performances and thus they run the risk of being taken out of context. In creating and negotiating self-presentations in mediated spaces, teens struggle to develop techniques to account for and adjust to these environments.

This chapter examines some of the ways in which teens negotiate selfpresentation and impression management in social network sites through the construction and maintenance of profiles. In analyzing these practices, I consider the ways in which teens alter their practices surrounding identity and impression management to account for the technical features of social network sites. My goal is to analyze how teens incorporate social network sites into their identity practices and how they work with and against the technology to meet their needs.

\subsection{Locating Identity}

The processes of self-presentation and impression management are intricately entwined with the concept of "identity," yet the term "identity" is slippery at best. Scholars have long argued about the meaning of this term as well as its psychological, social, cultural, and philosophical roots (Buckingham 2007; Gay et al. 2001). Countless theories have been posited as definitive approaches to identity and this continues to be an area of rich debate. 
Trying to locate identity, Buckingham (2007) maps five salient framings of identity that are specifically useful to consider when thinking about teens and new media. First, he looks at psychological or behavioral approaches in which identity is a developmental process. Adolescence is marked by the ways in which identity is formed, or at least put into crisis. Key scholars taking this approach include G. Stanley Hall, Jean Piaget, and Erik Erikson. Second, he turns to sociological approaches in which identity is marked through an individual's relation to society or culture. Issues of "socialization" shape this discourse, notably in studies of subcultures and the ways in which deviance and delinquency are seen as failed identity development. Third, Buckingham offers a notion of social identity best understood as "identification," in which an individual's sense of self is marked in relation to the group. Goffman's studies of self-presentation and impression management play a central role here. Fourth, he accounts for the concept of “identity politics," which emerges from the struggle over how identities are constructed by those with power. This body of scholarship destabilizes the notion of who actually wields the power to construct and control an individual's identity and is tightly wrapped up in discourses of class, race, gender, and queer. Fifth, he introduces how modern social theory approaches identity as being what Anthony Giddens (1991) calls a "self-reflexive ... project of the self” or what Michel Foucault (1990) might refer to as "self-monitoring."

In technical and legal discourses, the term "identity" is often employed to reference a unique person or body (Solove 2006). Technical systems often use 
"identity" to refer to the database placeholder or the set of demographic characteristics that uniquely identify someone. Legal discussions of "identity theft" and privacy also take this angle, using identity to refer to the collection of information that successfully references a unique individual.

There is a long history of scholarship on the intersection of technology (including the Internet) and identity, stemming from diverse theoretical and methodological perspectives and addressing different aspects of identity (Balsamo 1995; Castells 2004; Clippinger 2007; Donath 1999; Reed 2005). Some of the earliest work, such as Donna Haraway’s (1991a) "Cyborg Manifesto,” focused on how a mediated existence as a cyborg would result in new manifestations of identity, challenging systems of power by complicating embodied assumptions in identity politics. While Haraway's hypothesis prompted tremendous discussion and analysis, this utopic future did not emerge. Even when trying to be deceptive, people reproduce their embodied experiences online (Berman and Bruckman 2001).

One of the most prominent scholars examining identity and technology is Sherry Turkle. Her seminal texts The Second Self (1984) and Life on the Screen (1995) examine identity from a psychological perspective, focusing primarily on youth. Turkle uses psychoanalysis to consider the ways that technology helps and complicates identity development. She also posits that the fragmentation of identity enabled by technology takes identity crisis to new levels (Turkle 1995: 255-269). While she showcases and examines various ways in which youth use technology to 
work through identity, she frames these practices as acts of identity simulation. Her analysis assumes that online activity is removed from physical interactions. Additionally, her work focuses on early adopter youth and children who are just introduced to computing. While our topical interests are similar, I disagree with many of Turkle's conclusions. Even if her analysis holds for those who are trying to create separate "virtual" worlds through online engagement, the vast majority of teens are not. For this reason, I believe that the assumption that teens are fragmenting their identities through online engagement is inaccurate. That said, I do believe that they present a facet of their identity based on the social context involved (boyd 2002). I just do not believe that this creates the identity crises that Turkle suggests.

Like many of the scholars before me, I weave together multiple approaches to identity in my own approach. While my approach draws on multiple frameworks, Goffman's approach to the performance of self and negotiation of impression management are at the core. The frameworks that I purposely exclude are those that presume that identity is a set of prescribed and time-delimited stages; I do not subscribe to this view, although I occasionally address scholars who do. I see profiles as "digital bodies" in that they both uniquely identify a person and are the product of self-reflexive identity production. To me, profiles locate and are the combination of controlled self-descriptions in the context of social connections. As teens struggle with the ways they are seen and how they mark themselves in relation to those around them, I see identity work that combines the complex ways in which social 
norms, context, and people complicate acts of self-presentation and identity management. I see their work as introducing an entirely new set of identity politics by pointing out the ways in which the collapse of context can increase the challenges of those whose identities are framed by systems of power.

\subsection{Writing Identity into Being Online}

Analyzing the textual culture of an early online community, Jenny Sundén (2003: 3) argued that an online participant has to actively and consistently "type oneself into being" to exist and be visible online. Sundén's research focused on an early precursor to massively multiplayer online role-playing games known as a MUD (a.k.a. Multi-User Domain or Multi-User Dungeon). In this gamelike social environment, participants had to textually produce every aspect of the imagined world, from the chairs and tables in a room to the fashion accessories worn by individuals. They typed spaces into being and they also explicitly typed people into being. Attributes like gender are assigned to a person through the use of an @gender command.

In unmediated environments, it is easy to take bodies—and the roles that they play-for granted. By locating a person in space and time, a body signals presence by its very being. A body is loaded with cues about a person's identity; gender, race, and age are written on the body in ways that are often difficult to obscure. Through fashion and mannerisms, bodies can be used to convey a wide variety of attitudes, emotions, affiliations, and identity information. Bodies, in the traditional sense, do 
not inherently exist online. By default, a person's digital presence is little more than an IP address. While bodies in the corporeal sense are not present online, Sundén argues that the digital world is not free from the constraints of bodies of materiality for "the virtual does not automatically equate disembodiment" (Sundén 2003: 5). How people represent themselves and interact online is fundamentally influenced by their embodied experience.

In the environments Sundén was investigating, it was quite common for participants to have no face-to-face interactions with one another. Likewise, the norms in MUDs did not demand that participants model their online representations to accurately reflect their offline bodies. As such, the typed bodies in MUDs might not be "real" in the commonly understood sense. Such deviations are not considered deceptive in MUDs as these environments encourage identity play and participants do not assume that a textual performance is a sincere representation of the typist's unmediated body.

Conversely, for American teens, social network sites are not a distinct space that is constructed online and left as a virtual sphere of imagination. The performances, conversations, interactions, and context of social network sites are tightly entwined with other aspects of participants' lives. Teens move seamlessly between different mediated and unmediated environments and their participation in social network sites is typically inextricably linked to unmediated encounters. Teens participate in these spaces alongside people with whom they interact in unmediated 
environments. The performances that take place online are not isolated acts, disconnected from embodied settings, but rather conscious acts that rely on a context that spans mediated and unmediated environments and involves people who are known in both settings. While identity play was commonplace in MUDs, the profiles that teens create in social network sites are typically tightly connected to the identity that teens embody in unmediated environments.

The continuity between social network sites and other environments affects teens' practices concerning the creation of a digital representation. The digital bodies that emerge through profiles are tightly tethered to the individual behind the profile, if for no other reason than because they serve as a direct digital representation of that person for mediated interactions. While some suggest that identity work through the Internet tends to involve the creation of fictional characters unconnected from embodied reality (Turkle 1995), this was not a common practice that I witnessed. Some teens choose to depict an idealized self or present a facet of their identity that they do not normally show in public spaces, but few generate self-representations that are completely disconnected from their everyday lived experiences. More often, they are simply seeking to represent themselves in the most positive light possible.

The process of writing oneself into being online forces teens to work through identity in new ways. Teens must work out how they envision themselves and how they want to be seen and then they must use tools to formally articulate this, often 
without the feedback mechanisms and context that make impression management seamless. They must struggle with being misinterpreted and having those around them and the technology itself control how they are represented. Yet the ways that they manage this through the construction and maintenance of profiles sheds light on the intersections of identity and technology and the ways teens learn to do identity work in wholly new environments.

\subsection{The Art of Profile Creation and Management}

The mere act of creating a profile on a social network site requires some selfreflection, if only to consciously decide what to fill in or ignore when prompted with questions and forms. As documented with respect to many gen res of social media (Brake 2008; Ellison et al. 2006; Hodkinson and Lincoln 2008; Reed 2005), creatively constructing and updating a "digital body" requires participants to think about how they wish to represent themselves. Creating digital self-representations has become a common act for many teens. From choosing a representative IM screen name to updating a blog to maintaining a social network site profile, the teens I met often face pressure to be witty, entertaining, creative, or otherwise interesting while writing themselves into being online.

The pressure teens face in digital self-presentation is not wholly different from that surrounding fashion and image in unmediated contexts. How individual teens adorn themselves-online or off — signals valuable information about their sense of self and their social identity (Crane 2000; Davis 1992). Teens consume fashion as a 
direct form of self-expression (Piacentini and Mailer 2004) and they seek out fashion symbols that allow them to simultaneously fit in and stand out among peers (Milner 2004). Through the use of text, images and other media, and design, teens create profiles that signal information about their identities. The mediated selfrepresentations that they create reveal both what they share in common and how they distinguish themselves from those around them (Liu 2007). Creating a profile is an act of impression management, requiring teens to consider who might see their profiles and how they might be interpreted. The desire to be seen in a positive or accurate light compels teens to carefully craft their profiles.

\subsubsection{Techniques for Self-Presentation}

Crafting the perfect social network site profile is an art. Choosing photos, selecting songs, creating layouts, and determining how to fill in various text fields takes time because teens consciously consider the impressions that their profiles might leave. Dom, a black 16-year-old in Washington, told me that carefully choosing what content to put on his profile was important to him. Explaining why, he said, "I chose what I wanted on my profile because I thought it represented me well." Dom wanted his profile to make a good impression on those who saw it. For Dom, this meant creating a profile that centered on his friends and his music. Dom and his cousin scoured the web for layouts until Dom found one he liked. He then Photoshopped a picture of himself and used that as his background. He added photos of his friends and uploaded songs he created so that people would get to hear his 
music when they visited his site. Through the combination of a funky layout, photos of himself and his friends, and the streaming of his music, Dom was able to create a self-representation that he thought conveyed who he is and what mattered to him.

Social network sites-and MySpace, in particular-are structured in a way that assumes the viewer does not know the person behind the profile, yet most teensincluding Dom-are primarily using social network sites to interact with friends and peers (Lenhart and Madden 2007b). In crafting their profiles, these teens are not trying to explain themselves to strangers but rather to create a digital selfrepresentation that will be well received by people they already know. This drives what they choose to put on their profiles and motivates them to repurpose features of MySpace that were designed to help strangers meet.

As discussed in more detail in Appendix 2, teens input six types of content to create a MySpace profile: photographs, demographic information, lists of tastes and interests, open-ended areas for self-description, music, and designed layouts. Additionally, as their list of Friends and those Friends' comments are displayed on teens' profiles, the content their Friends choose to contribute helps shape a teen's profile.

Sections like MySpace's About Me are structured for individuals to explain themselves, presumably to strangers. This open-ended section is centrally displayed in profiles, but many teens feel no need to describe themselves to those who already know who they are. Traviesa, a Hispanic 15-year-old in Los Angeles, goes so far as to 
use this section to make it clear that her profile is intended for those she knows. She uses her About Me to state, "Hey, my name's Traviesa ... but most all of you know me anyways so just talk to me and stop being stupid." Her profile is publicly visible and she knows strangers might stumble on it, but she believes only those who know her would have any reason to dive deeper. Given this, she does not think she has to go into detail about who she is, although she happily offers a list of her favorite music.

The About Me section is the most prominent open-ended field on a MySpace profile and many teens repurpose this for various forms of self-expression. Some teens display videos that they wish to share with their friends. Others—primarily girls-use this space to display quiz or personality test results. Some teens write poems or display a list of favorite quotes. Some leave it blank and others, like Allie above, take the challenge seriously and try to describe an aspect of their identity. Surfing MySpace, I saw a variety of different acts of self-expression. One teen posted a long rant, followed by: " $i$ dont try and act smart to others / $i$ just state the facts in which i know / also, im very up front / handle." A teen boy used this section to write a love poem to his girlfriend, exclaiming his love and promising to be there forever. Elsewhere, a girl offered a list of facts about herself, including "I love duckies!" Biblical passages and song lyrics are common, as are photographs of oneself or the teen's friends. While these various moves may not directly address the About Me query, they all reflect information about the teen being represented. 
Another open-ended section that teens regularly repurpose is "Who I'd Like To Meet." Based on the MySpace profiles I viewed, most teens treated this section in a similar fashion to the About Me section, preferring to leave it blank, add multimedia or quizzes, or otherwise use it as a continuation of the About Me section. Many of those who actually addressed the topic explicitly defined the intended audience: "I would only like to meet friends on here." A few mentioned that they would be open to meeting interesting people or people who shared their interests, but this was not common. More often, teens indicated that they would like to meet celebrities, bands, or public figures like the Dalai Lama and the pope. While this profile element was designed to help people indicate those they would like to meet through the site, choosing to list well-known people is primarily about marking identity through fandom.

Rather than describing themselves, some teens use open-ended fields to express their feelings about those around them. On one profile, a girl detailed how much she loved her friends in her About Me and on another, a boy wrote a R.I.P. message to a friend who had died. Michael, a white 17-year-old from Seattle, told me that his profile focused on two things: football and his girlfriend. When we met, his About Me began with "I love my girlfriend AMY." Likewise, Amy, a black-white 16-yearold, filled her profile with odes to Michael. They both showcased their relationship through blog posts, photos, and comments. Like many other teens, Amy and Michael visibly performed their relationship using profile sections intended for selfdescription. A few weeks after I met Michael and Amy, they broke up. While any 
sign of Michael disappeared from Amy's profile, Michael made this change in relationship status quite visible. Where there was once a proclamation of love, Michael's profile displayed, "I hate my stupid bitch ex girlfriend." Whether favorably or not, teens regularly mark who they are in relation to those around them and their self-presentations are framed by their relations to and opinions of others.

Using photographs of friends as a form of decoration is not unique to the Internet. When I interviewed Gabriella, a Honduran 15-year-old, at her school in Los Angeles, she came into the room holding a binder spilling over with paper. The front, back, and side of the binder were decorated with a collage of cut-up photographs of her and her friends artistically positioned. I asked Gabriella about the binder and she told me that she liked having her friends with her at all times. Gabriella's choice in clothing, accessories, and makeup made it clear that she took fashion seriously. Her fingernails were painted as black-and-white dice and she told me that she had them redone regularly. Gabriella took her online profile just as seriously as her clothing. She told me that she actively sought out interesting backgrounds and changed her profile weekly. That day, the theme was checkers. In visiting her MySpace, I found parallels between her profile and her binder-both were covered with photographs of friends.

While teens have fun with most self-description and interest fields, their responses to the taste fields (e.g., favorite music, TV shows, movies, etc.) tend to be more serious and more complex. Rank ordering "favorite" media is not simple for 
many teens-tastes change through time and many people do not think about their tastes as abstract lists. Tastes, like fashion, are rooted in and constructed by social systems. People distinguish themselves by their tastes and tastes are one way in which social distinctions are made (Bourdieu 1984). When asked to articulate favorites, many people—consciously or unconsciously—select tastes that will signal the right impression (Donath 2007). In other words, they try to position themselves in relation to others through their taste choices. Analyzing MySpace profiles, Liu (2007) found that social structures drive taste performances. In a surprise twist, people were more likely to list tastes that were different from those of their Friends. Yet this performative differentiation does not imply that people no longer share tastes with those around them. Rather, it is quite likely that the act of public articulation motivates people to list tastes that differentiate themselves from those around them.

The self-descriptive text, media artifacts, and designed layouts are only one aspect of a profile on social network sites. Another key component is the public articulation of social connections through the Friends lists. Because these connections are publicly displayed on a teen's profile for all to see, they serve as more than just a buddy list or address book. These connections-and the comments left by these Friends-shape teens' digital self-representations. In other words, social network sites formalize the adage that "you are who you know." While teens control certain content on their profiles, they do not control the photos or names their Friends select, although these are displayed on their profiles. They may delete 
comments that Friends leave, but most often, they do not. On Facebook, teens can also post and tag photos, which are automatically connected to their profiles without their permission. As such, what teens explicitly state on their profiles is only one part of their self-presentation online. Their profiles are heavily co-constructed by those around them.

Network effects also play a significant role in how teens construct their profiles. As teens surf each other's profiles, they get a sense for what is common among their peer group and they often craft their profiles to reinforce these norms. Girls who have "sexy" photos tend to have Friends who also choose this style of photo. Teens who dedicate their profiles to Jesus tend to have Friends who also publicly display their faith. When surfing profiles, it is not possible to tell who set the norms, but the clusters are visible. While the general tone of a profile tends to be consistent within social groups, the actual content is rarely replicated. For example, while extensive MySpace layout modification may be consistent across a Friend group, no two Friends would use the exact same layout. With both profile styles and tastes, teens tend to differentiate themselves through specific content even when the general tone or taste genre resembles that of their Friends. In other words, each profile is unique, but there are network effects in terms of tone, genre, and style suggesting that teens are positioning their digital identities in relation to those around them. This practice is akin to fashion in unmediated settings, where it is common for teens to choose clothing that is generally of the same style as their peers but where wearing the exact same clothes as their friends is taboo. 


\subsubsection{Bedroom Culture and Fashion}

The way in which teens adorn their online profiles parallels how they decorate other spaces and material objects that they control—school lockers, backpacks, bedrooms, and their bodies. The photomontage style that Gabriella, the 15-year-old from Los Angeles, uses for her binder mirrors how many teens decorate their lockers and bedroom walls. Teens have long stitched together media artifacts as a form of self-expression, placing them on spaces or objects that are connected to them. Likewise, clothing and accessories have long been a way for teens to mark their identities in relation to cultural dynamics and people. As teens craft their profiles, they combine both of these practices, revealing the ways in which profiles are both like and unlike their physical counterparts.

For teenagers, and especially teen girls, bedrooms and their postered walls have long been a space where cultural participation and identity are manifested and media of all forms has played a central role in this process (McRobbie and Garber 1976; Steele and Brown 1995). McRobbie and Garber's (1976) initial introduction of "bedroom culture" focused on the ways in which teen girls consumed culture, although critiques have emphasized that teens actively engage in cultural production in bedroom culture, especially when media is involved (Kearney 2006; Lincoln 2004, 2005). What teens create through their choice of decoration and memorabilia are both social spaces and self-representations (Lincoln 2004). 
As teens move toward networked publics, they take bedroom-culture practices with them and networked publics can be seen as "virtual bedrooms" (Hodkinson and Lincoln 2008). Digital self-representations are equivalent to the bedroom walls where teens exhibit their identities and the social spaces that are created are both like and unlike bedrooms. Mixed media is used in both environments, but the media that teens use in bedrooms is primarily static, while the content they display on their profiles can be interactive, animated, and linked. It may be cheaper to display media on profiles than in bedrooms, but the time to find and combine such media may be much greater.

More than anything, the difference between bedroom walls and profiles is the scale of the audience. Teens may show their bedrooms off to their friends, but they rarely have the opportunity to invite their entire cohort over to see their decorations. While the potential scale of interaction online is far greater than in a bedroom, Livingstone (2008) found that there is still an expectation of intimacy; teens deliberately choose what to share based on their understanding of the social situation and technical context. Teens approach social media environments with a view of privacy that is primarily about having control over the situation (Livingstone 2006). Quoting Giddens (1991: 94), Livingstone (2008: 471) reminds us that "intimacy is the other face of privacy." It may seem paradoxical, but teens seek to be simultaneously public to some audiences and private to others (Livingstone 2008: 471). In this way, they work toward a sense of intimacy and control that parallels bedroom culture. Many of the teens I interviewed noted that their bedrooms were 
not quite private because their parents and siblings entered when they wanted, but at the same time, they felt as though these spaces were not quite public either because they had some sense of control and not just anyone could or would come walking in. With social network sites, teens may understand that they are visible to broad audiences, but they do not see their participation in networked publics as being universally public.

Social media does not necessarily replace bedroom culture, but it is sometimes a meaningful alternative, especially for teens who lack control over their physical environments. For example, after a talk I gave in New Jersey, a girl told me that she liked decorating her MySpace profile because it allowed her to be creative. She was not allowed to alter her bedroom after her interior decorator mother had designed it, but she could do as she pleased with her MySpace profile. She relished the opportunity for creative self-expression and changed her profile regularly. While this is an extreme case of noncontrol, many teens are restricted in what they can and cannot put on their bedroom walls or in other spaces where they might have once marked identity. Many of the schools I visited no longer have lockers and many of those that do restrict locker decorations- "for fire safety reasons."

Fashion is another site where control for self-expression is fraught. Clothing and backpacks continue to be a battleground, especially when it comes to schools. Likewise, parents still try to limit what their kids may wear. Fashion plays a significant role in the marking of identity (Davis 1992) and, as I will discuss in the 
next chapter, status (Crane 2000; Piacentini and Mailer 2004). Teens use fashion to mark themselves in relation to each other (Milner 2004) and identify with social groups (Hebdige 1979). Clothing and accessories become tools for self-expression and teens dress themselves as a form of identity work. Yet while teens value fashion's symbolic opportunities for self-expression and identification (Milner 2004; Piacentini and Mailer 2004), adults worry about visible markers of teens' resistance to adult norms and reinforcement of social hierarchies. Dress codes are relatively common and often hailed by parents as healthy approaches to curb gang violence, status mongering, and conspicuous consumption. While fashion and its relation to status and peer groups is discussed in greater detail in the next chapter, it is important to note that fashion still operates as a key mechanism of self-presentation and it plays a core part in impression management.

As adults seek to control the ways in which teens can engage in acts of selfexpression, teens seek out new spaces, including the Internet. Because profiles are both a representation of an individual and also a space for social interaction, the practices of self-expression that take place parallel both bedroom culture and fashion.

\subsubsection{Varying Degrees of Participation}

Although creating a profile is necessary for participation, actively decorating it is not. Teens often feel social pressure to upload a photo and put some effort into creating their profiles, but there is less pressure to regularly update the style and 
layout. At the same time, teens often do not want to let their profiles get stale because they think that this leaves a bad impression. The desire to keep a profile fresh often drives updates. Nick, a black 16-year-old with Native American roots from Los Angeles, updates his pictures and backgrounds every few months because otherwise "it gets real boring... I'll log on to my profile and see the same picture every time. I'm like man, I'm gonna do something new."

While some teens are motivated to update their profiles constantly, others never update them. Some, like Shean, a black 17-year-old in Los Angeles, approach MySpace from a functional, communicative perspective. "I'm not a big fan of changing my background and all that.... As long as I keep in touch with my friends or whatever, I don't really care about how it looks as long as it's, like, there." His attitude is fairly common, although boys more frequently express this than girls. In my interviews, more girls felt pressure to keep their profiles up-to-date and expressive, rich with photos and new content. When I browsed profiles, it also appeared to be girls who updated more frequently.

Not all teens felt pressure to create the perfect profile or update it often, but even among those who did, not all conformed. Just as some teens resist pressures to conform to other normative peer culture practices, some teens also eschew the construction and maintenance of profiles online. Much of their resistance stems from the general frustration that participation requires conformity to broader peer culture attitudes, social hierarchies, and value sets. When marginalized teens opt out 
and critique these practices, it is often an explicit move to distance themselves from the unattainable status hierarchies of the "popular" people. Their critiques, while often warranted, challenge the practices of identity work and status negotiation that take place through the construction of profiles. While I interviewed teens who eschewed these practices, none were as articulate and descriptive as Cara, a 20-yearold from Maine. Rich with sarcasm, Cara lamented the ritual of profile construction on her blog:

"Really I'm only doing anything to construct an ideal identity though, and so everybody knows how emo and special I am. It's the same reason I have a LiveJournal, and a MySpace, so that I can stare at pictures of myself and tweak my layout until I feel satisfied with my feigned attempt to digitally define myself. I have a better idea, I'll just cease all activities that could in any way possible suggest that I'm whoring myself out to some stereotyped image, and quit trying because no expression is ever accurate enough to encompass all my human complexities... God forbid other people think I was faking myself or degrading the sum of my substance down to a list of my favorite movies and a few choice photos."

Cara's sarcastic take on the process of profile development and maintenance gets to the heart of why teens engage in these practices. Many teens want to create a digital representation that successfully represents who they are and is well received by those around them. Yet to do so through a social network site profile requires 
fitting oneself into a set of predetermined boxes and lists of tastes. The result is coarse, and not always representative, adding to the social uncertainty teens experience when they face their peers.

Profile creation forces teens to consider how they want to represent themselves. In working through this process, teens account for who they think will see their profiles. Their decisions about what to say are deeply connected to their sense of audience. That audience, by and large, is their friends and what teens say is best understood in that context. Out of context, some of what appears is not quite what it seems.

\subsection{Self-Presentations in Context}

Profiles and other acts of self-presentation are not performed in a void. Decisions about what content to display are situated in a context that is driven by the space, social situation, and people. In mediated environments, technology helps shape context, but technology alone does not define the context. For the teens I interviewed, other people were the most important factor in their decisions of how to present themselves. They were concerned with who they thought should, would, or could view their profiles. The context in which they were operating was shaped primarily by those they imagined to be their audience and how they related to that group. Likewise, in performing toward this imagined audience, teens were trying to define the social situation by rooting it in that context. 
Teens typically target their profiles at their friends. On multiple occasions, teens quoted the byline from MySpace's website: "MySpace is a place for friends." This intended audience is most visible when teens worry about being well received, a dynamic that is mapped out in Chapter 5.

When pushed to define their audience, teens often focused on who they thought should not be viewing their profile. By and large, teens emphasized that adults were not part of their intended audience. For example, when asked if she thought her teachers were on MySpace, Traviesa, the 15-year-old from Los Angeles, responded by saying, “That's nasty!” Aria, a 20-year-old college student from California, took this sentiment one step further, noting, "I don't really believe that 'online social networking' is something you can do with someone whose genetic material you inherited without subverting the laws of nature." The challenges of negotiating profiles with parents and other adults are discussed in greater detail in Chapter 6.

Speaking to an imagined audience is not new. Writers, politicians, and TV actors have long performed to an imagined audience, driven by who they think is watching and who they wish might be. These people learn how to control their selfpresentations before imagined audiences as a part of their professions, but these acts are always situated in public contexts. For teens negotiating networked publics, navigating imagined audiences is a part of everyday life. Unlike professionals seeking to address the public at large, teens are not focused on situating their acts broadly. While their potential audience might be global, their imagined audience is 
very local, primarily consisting of people whom they know. Yet teens must also face many of the same complexities that public speakers face in crafting their selfpresentations, in part because of the potential visibility of their acts.

In No Sense of Place, Joshua Meyrowitz (1985) maps out the ways in which electronic media—and particularly television—affects social situations, impression management, and self-presentation. He focuses on the ways in which electronic media collapses spatial boundaries and social contexts, blurring social roles and bringing audiences together who might not normally be co-present. Because of electronic media, information and social acts lose their context and new identities, behaviors, roles, and social situations must be formed to account for the way that social structure is changed. This, in turn, disrupts public and private distinctions, boundaries between social groups, and the very essence of public life. Writing before the Internet had captured mass attention, Meyrowitz was before his time. What he demonstrates with respect to television and other early forms of electronic media has only intensified since the rise of the Internet. The complexities that he documents regarding what public figures face in managing social contexts are now part of everyday life.

While teenagers leverage Friending features to construct an imagined audience toward which to target their self-presentations (boyd 2006b), who is watching might not always be who teens expect or want. While some teens fear the presence of strangers who have malicious intentions, many teens accept that average strangers 
might happen upon their profiles, just as average strangers pass them in the streets, but they assume that these strangers will walk on by. Thus, they do not account for average strangers in choosing how to present themselves. For example, Kiki, a blackwhite 16-year-old from Kansas, originally made her profile private because, as she explained, "I don't want nobody on my profile." In other words, she did not want strangers to be there. Yet once she was familiar with the site, she decided she should change her profile to make it public. She realized no one would visit her profile and so she stopped caring about locking her profile down. Still, she does not want to accept Friend requests from strangers, but getting requests does not bother her; she just ignores them.

In trying to balance different potential audiences and attract the ones that they desire, teens focus on dissuading unwanted visitors and crafting profiles that appeal to their peers, even at the expense of upsetting the audiences that they do not want. This move can be risky, especially if their profile content is upsetting to those who hold power over them, because the technology easily collapses the boundaries that allow teens to distinguish context through social group. The challenges teens face with respect to balancing different audiences mirror those Meyrowitz (1985) described with respect to public figures' facing television and radio. Negotiating multiple audiences creates context collisions and teens feel more pressure when they are forced to contend simultaneously with different audiences, such as peers and parents. 
In an effort to control the context of their self-presentation, teens take two different tactics. First, they use structural means like providing false information to make themselves unsearchable or using privacy settings to limit who can access their profiles. This first approach is "security through obscurity" and the teens I interviewed recognize that it is not foolproof, but they believe that it is a good first measure to dissuade teachers and college admissions officers from finding their profiles. Parents intent on finding a teen's profile are a different story, but teens believe that privacy settings are generally effective against them as well as against the nosy teacher or school bureaucrat.

Second, teens try to define the social situation through explicit and implicit acts to control the audience. They use Friends lists to make it clear whom they see as their intended audience; this is reinforced by privacy features that lock out all others. They also make social demands that parents and other adults stay out, using "Keep Out" language that mirrors fights between parents and teens over bedroom access. In this way, teens try to control access to the space to define the context. Yet this is often ineffective, in part because technology makes access appear public. In countless communities, I have heard teens try to dissuade parents from accessing their visible profiles by declaring, "But it's my space." In responding, "But it's public," parents typically miss the point that teens are making, for their sense of privacy has to do with context and control, not potential access. That said, this fight often follows the same logic of older teen/parent fights over space, context, and privacy that include arguments like "but it's my bedroom" and "but it's my house." 


\subsection{Performing Falsehoods-Deception, Play, or Control?}

Some teens seek to create rich profiles, while others maintain profiles that provide little information. Yet among both groups, uncountable teens respond to requests for name, age, location, income, and other demographic information with responses that do not accurately reflect the teen's "true" identity. Sometimes what they list seems slightly off; other times, the misinformation reads as absurd. For example, some teens say that they are 100 years old or make more than $\$ 250,000$ in income. At one level, this content can be seen as fraudulent. At another, it looks like identity play. Neither of these theories account for why teens tend to put "inaccurate" information on their profiles. Teens do not view these acts as deceptive, because those for whom they intend their profiles know their real names, ages, locations, and hometowns. They may see their responses as humorous, but they are not trying to create an alternate identity. They are simply trying to structure their presence in a way that allows them to be visible to those who matter while being invisible to those who do not. For example, in explaining his practice of putting fake information on his MySpace, Mickey, a Mexican 15-year-old from Los Angeles, says, "It's not that I lie on [MySpace] but I don't put my real information. Like I put my real information under 'About Me." For Mickey, fake content is not the same as lying because those who know him can see that it is him. 


\subsubsection{Motivations for Providing Inaccurate Information}

Teens lie about their ages for a variety of reasons. Some are simply trying to be funny or entertaining, such as those who indicate large incomes. Others seek to fool strangers who might be interested in engaging with them. Responding to adult fears concerning safety, many teens believe that obscuring their ages and other identifying information will decrease the possibility of harm. Another group of teens alter their ages to circumvent technical and legal restrictions. They lie because age equals privilege in online settings. They lie because doing so is the only way in which they can gain access to the technology that they desire. They lie to circumvent barriers to entry that they disrespect. And, often, they lie with their parents' knowledge.

Roughly a quarter of the teens I spoke with had profiles that indicated a false age. Some lied excessively, indicating that they were 101,69 , or another postretirement age. Penelope, a white 15-year-old from Nebraska, explained that she chose to list her age as 100 because she thought it was "funny." Others indicated that they were over the socially constructed "legal" ages of 18 or 21 . Some told me that they listed these ages because they thought that MySpace restricts minors' accounts. One girl said she thought it was better to be older than 18 because this would "keep the child molesters away." (Ironically, many adults worry that teens pretending to be older are more likely to be vulnerable to those with malicious intentions.) Another girl told me that she listed her age on her MySpace to match her fake ID in case anyone 
checked. A third group lied arbitrarily, indicating that they were anywhere from three years younger to eight years older than they were. When I asked them about their decisions, most shrugged and responded with "I don't know." One boy told me that he scrolled down the list of birth years and chose one randomly. The teens I interviewed did not take their profile ages seriously or think that they signaled anything important, even those who had indicated that they were older. Some did tell me that other teens at school marked themselves as older to look "cool," but this was never an explanation I was given by those whose profiles marked them in the cool age range of 18-25, and I doubt that those who arbitrarily chose ages older than 40 were actually driven by looking cool when they told me that they did not know why they chose that age. The closest example I experienced of someone's taking a profile age seriously was a teen who had gotten on Facebook as a high-school student when it was college-only because his father had given him an .edu address; he said that he marked himself as older because college students are older and he did not want to stand out.

Among other cohorts or in other contexts, age inaccuracies mean different things. In online dating sites, adults frequently shave off a few years (and a few pounds) to appear desirable to potential suitors (Hancock et al. 2007). In this context, adults are intentionally engaged in deceiving other people. Teens do sometimes change their ages as an intentional act of deception, but their goal is typically to deceive companies whose websites have age restrictions or to discourage unwanted attention from strangers. From their perspective, the people they care 
about (e.g., their friends) already know how old they are and do not require a system to inform them. For teens, it is more important to be accurate about birth dates than birth years so that when the systems remind their friends of their birthdays, there are no awkward situations. When it comes to peer groups, the social consequences of providing inaccurate birth-date information is far greater than of providing inaccurate birth year.

While teens have various motivations for providing inaccurate information, it has become a common practice. Most teens who engage in these practices are not looking to deceive but to leverage the technology to meet their needs.

\subsubsection{Legal and Technical Limitations}

In 1998, the U.S. Congress passed the Children's Online Protection Privacy Act (COPPA). Intended to protect children from marketers, COPPA restricts websites from collecting information from children under 13 without verifiable parental permission. It also includes provisions for when websites must provide privacy policies and rules for protecting the safety of children under 13. Because age verification is a technical and privacy nightmare, most websites comply by barring all those who self-report that they are under 13 from creating an account. The most common approach taken by websites is to limit the list of birth years in the scrolldown menu to current year minus 13. COPPA did not stop most children from creating accounts, but it did teach children and their parents an important lesson: Lying is the path to access. 
Many of the teens who I interviewed learned to lie about their ages in middle school, when they were younger than 13 . When I asked teens how they learned to change their birth year to get access, parents and older siblings were most frequently the culprit. Once, when I was talking to a mother/daughter pair at an airport, the girl told me that when all of her friends were getting onto AOL's instant messaging (AIM) service, she approached her mother about being too young to create an account. Her mother sat down with her at her computer and showed her how to change her birth year during the sign-up process. Her mother confirmed this account, noting that she thought that the limitations were "stupid" and that technology companies should not be in the business of parenting. These two were not alone-I heard similar accounts across the country and, for the cohort I interviewed, AIM was the gateway to age deception. Most joined during early middle school, typically with their parents' knowledge. By the time they joined MySpace, they were already accustomed to lying about their ages and did so regularly.

I did not interview teens who were too young to be on Facebook or MySpace, but there are unquestionably countless underage youth using these sites. In a Boston focus group run by members of the Berkman Center's "Digital Natives" project, 12year-old Tom declared that he was on Facebook even though he was not technically eligible because"there's no way of proving age. You can go on any site and say, 'I was born in 1981, and I'm 18 now. '” He found the age limitations ludicrous and wanted them to end, but 'even if the age limit isn't brought down, there's still people like me. 
I'm not technically allowed to be on Facebook yet. But I mean I can say I'm 14 now." Tom lies about his age because he can and because this gives him access to a site that he believes he should have the right to access.

COPPA was designed to protect children's privacy and keep marketers from going after them, but it has been increasingly renarrated as a safety mechanism. The teens I interviewed knew nothing of the intentions behind the restrictions and simply saw it as a tool of control. Thus, they assumed that any site that made them provide their ages would limit their activities depending on what they selected. Like many sites, MySpace offers different features for users based on their ages. Unaware of the differences, many teens assumed that accounts for minors were likely limited or "crippled," prompting them to mark themselves as older. Ironically, accounts for minors were initially more advanced than adult accounts—-minors were offered options for privacy while adult users could not set their profiles to private. There were also limitations, but typically they protected teens' privacy. While teens were lying that they were older to avoid restrictions, adults started lying that they were younger to gain access to additional features.

\subsubsection{Safety through Inaccuracy}

Teens do not provide inaccurate age information to circumvent technical limitations. Across the country, adults regularly encourage teens to lie to avoid potential predators and most of the teens who I spoke with believed that providing 
inaccurate information was an important step toward safety. ${ }^{8}$ Law enforcement officers regularly give school assemblies where they encourage youth not to provide identifying information to public websites. In Michigan, white 16-year-olds Bianca and Sasha recounted a school assembly by police officers at which the officers tried to scare teens away from Facebook and MySpace by telling them horror stories of terrible incidents. What Bianca and Sasha took away from this event was that teens who got hurt were "stupid" and being "smart" meant not putting up real information. Parents and teachers replicate this view, encouraging teens to explicitly lie about who they are and where they are from so as to avoid being stalked. The teens I interviewed were far less likely to provide inaccurate information on Facebook than on MySpace, most likely because they believed that Facebook was safer than MySpace and that they were less at risk.

For similar reasons, many teens lie about their locations. While teens in major urban centers like Los Angeles typically provided accurate hometown information, teens in smaller town or suburbs often did not. Some listed only their states, but many more chose nicknames for their towns like "Nowhere," "somewhere ur not," and "Hicksville." Others lied about their states or countries. When I began analyzing teen profiles, this practice appeared widespread. While it is impossible for me to verify the locations of teens I did not interview, it seems unlikely that so many

\footnotetext{
${ }^{8}$ While this approach to safety is common and widespread, there is no data to show that the elimination of identifying information actually does reduce risks of molestation, abduction, or harmful encounters with strangers (Wolak et al. 2008).
} 
teens in Afghanistan and Zimbabwe are connected and active on MySpace. What makes their location information especially suspicious is that these users often appear white, list U.S. high schools, and have friends who are primarily from a single small town in the United States. While some of these teens may indeed be studying abroad, it is more likely that they chose these countries because they are the first and last options in the pull-down menu of countries. Providing inaccurate location information is particularly common among teens who worry about safety and parents encourage this practice.

In further efforts to obscure identifying information, some teens post fake names or put up abstract images or photos that are unidentifiable. In addition to avoiding potential predators, teens who did this were often trying to avoid parents, teachers, and other adults who knew and held power over them. In other words, they were using "security through obscurity" to achieve privacy.

Aaron, a white 15-year-old in suburban Texas, has mostly fake demographic information on his profile. His MySpace lists a fake name referencing media culture and indicates that he is older than 80 and from Azerbaijan. His profile is private and his primary photo is clearly Photoshopped. When I asked him about his photo, he said, "I turned it into a negative so if you know me, you'd probably be like, 'Oh, yeah, that's him.'” Not having a real photo reduces the risk that a stranger could find him. It is virtually impossible to tell that this profile is Aaron's unless he has made you his Friend. Yet, once Friends, it is possible to see his About Me, where he clearly states 
that this profile belongs to Aaron. Aaron is not trying to fool his friends. Instead, he is trying to decrease the likelihood that his profile will be found by his parents, teachers, and any stranger who may wish to contact him. He wants his MySpace to be available only to friends from school and church and he does not worry that they will misinterpret his age or location, although he said that a few have playfully teased him about his choices. The photo he chose is to help those who wish to Friend him confirm that it is indeed him while being unrecognizable to those who are simply scanning profiles. Still, he expressed doubts about this photo because he knew that parents and teachers would also recognize him and he did not want this. Shortly after our interview, he changed his primary photo to be that of a cartoon character.

Nothing about Aaron's profile is risqué or embarrassing. He has a few funny YouTube videos and some cartoon-related images, which, while copyrighted, suggest nothing other than an appreciation for this form of art and the culture around it. His photos are all G-rated and mostly consist of snapshots of him goofing around with his friends. His written content suggests that he is passionate about music and sports, has a lot of school spirit, is antidrug and antialcohol, and really believes in his church. The only thing he includes that might offend someone is his view on the war and a banner for a political candidate whom he supports. I should note that his Friends' comments are more colorful, including foul language and speech that could be interpreted to be attacks on Aaron. From Aaron's point of view, this is just how his friends talk with one another. This is consistent with other studies that find that 
most MySpace profiles contain some swearing and that swearing appears to be common for young people in MySpace (Thelwall 2008).

Scholars note that antiprivacy advocates often argue that the only people who want privacy are those who have something to hide (Solove 2007). Aaron is hiding, but it is not because he has something to hide. Aside from a potential misinterpretation of his Friends' comments, Aaron's profile reveals a healthy teen who is engaged and passionate about school, activities, politics, and God. He chooses to be private to prevent unwanted contact both from those who know him and might misinterpret his participation on MySpace and those who do not know him and might have malicious intentions. While he does not think that he is doing anything wrong, he does not want his mother to know about his profile. He is concerned that it would worry her because she regularly tells him about the terrible reports about MySpace that she hears on the news and how she is generally concerned about the potential that Aaron might be abducted. He has not lied about his account because she has not asked, but he would rather her not find out through searching.

Providing inaccurate information is one of the primary mechanisms by which teens attempt to control access to their profiles. They believe that inaccurate information makes it much harder for those who hold power over them and those with malicious intentions to find them. Teens have been socialized into practices of online deception and they have internalized safety warnings such that they believe 
that providing inaccurate information makes them safer and wards off unwanted contact. Likewise, teens have little incentive to provide accurate demographic information when their primary audience is their friends. Locating on eself in "Hicksville, USA" is seen as entertaining, not deceptive.

In constructing their social network site profiles, teens are forced to contend with conflicting views of how profiles relate to identity. Many adults, and the technology itself, expect teens to construct a digital identity that is linked to an embodied identity through articulated demographic information and identifying information. Honesty involves providing such data and constructing one's profile around such information. The logical extension of this viewpoint is that all other inaccurate information is either identity play or an act of deception. While this viewpoint is quite common among adults, teens do not share it.

Teens approach profiles and their digital identity from a different vantage point. Because preexisting social networks that bridge online and offline environments define the social context, teens feel no need to provide demographic or identifying information. As they see it, this information is already available and understood by those for whom it is relevant. As Dominic, a white 16-year-old from Seattle, explains, "Because all my Friends are actually my friends, they'll know if I'm joking around or not." Teens are not interested in providing the systems with identifying information simply to be honest. They do not see this as a breach of ethical behavior, but they do believe that providing such information can put them at risk 
later. When building a profile, teens are not constructing a census dossier-they choose content that helps them define the social situation and express themselves in that context.

\subsection{Controlling Access: Public or Private?}

In unmediated social situations, people tend to know who is present to witness a social act. This is not often the case in networked publics where audiences are invisible and access is asynchronous. Physical limitations help control the boundaries of unmediated environments-walls define the space and expressions can be witnessed only in hearing or visual range. Online, boundaries are porous-search collapses contexts, replicability allows traces of social acts to be copied to other spaces, and the persistence of data means that acts performed are not bounded by ephemerality. In other words, trying to keep social acts to one space online is futile, even though that is the norm in unmediated environments. Teens center their understanding of context on other people. Lacking the ability to control context and audience by confining social acts to particular spaces, teens must define the situation by controlling the potential audience. Inaccurate data may prevent searching, but it is not an effective tool for controlling access. Instead, teens leverage privacy settings to limit access to specific people, reinforcing the connection between audience and context in networked publics.

Controlling visibility through privacy settings varies by site. On MySpace, profiles are either "public" or "private"-profiles that are "public" can be viewed by 
anyone, while only an individual's Friends can access "private" profiles. Facebook is a bit more complex because its privacy settings involve high school and neighborhood networks. Teens can make their profiles visible to just their Friends or they can adjust the settings to involve everyone in their networks. Facebook allows teens to adjust settings by module. Since my fieldwork ended, Facebook has added additional layers of privacy, including the ability to make groups of people and control elements by groups.

All of the teens who I interviewed were aware of privacy settings, but not all knew how to use them or what the options meant. Nick, the 16-year-old in Los Angeles, told me that when his brother's girlfriend created his profile, she set it to private and he has yet to figure out how to change this. While some teens may not know how, 66 percent of those interviewed by Pew in 2006 (Lenhart and Madden $2007 \mathrm{~b})$ reported that they limit access to their profile in some way. This is particularly impressive given that users frequently do not understand privacy options, let alone adjust the default settings (Lederer et al. 2004).

While teens do appear to limit access, I found that their perceptions of visibility did not always match with reality, particularly with respect to Facebook. Because Facebook is perceived as the safer alternative to MySpace, few teens bother looking at the privacy settings there. At a private gathering after I finished my fieldwork, I was talking with a teen girl about the differences between MySpace and Facebook and she told me that Facebook was much more private because only people you 
knew could see your profile. Logging into Facebook, I joined the town network she was in, accessed her profile, and showed it to her. She was shocked-she had not realized that joining a town network made her profile visible to people who claimed to be from that town. I have been able to repeat this "party trick" on multiple occasions with both teens and adults. While there is no data on how many teens join town networks or how many of those do not realize how visible their profiles are, I was able to access many teen profiles by joining town networks across the country and I sensed that few of those I looked at, and teens in general, realized this. Teens throughout the country told me that they thought Facebook is safer than MySpace because of how it handles privacy. When asked why, Sasha, the 16-year-old from Michigan, told me that more controls meant greater privacy, while black 15-year-old Kaleb, also from Michigan, told me that he did not know why but he "just got that feeling." In fact, both of these teens had Facebook pages that were more visible than a Friends-only MySpace page would be.

Teenagers who use MySpace are far more fluent in the language of privacy settings and most have consciously chosen to make their profiles public or private. The teens I met who had public profiles had chosen this route intentionally. Some teens left their profiles public so that they could more easily be found. Sabrina, a white 14-year-old from Texas, explains that being public is the best way to be found by friends. She says, "I have searched some of my friends by their name, but if [their profile is] on private, then I can't really find out if that's who they are." In Los Angeles, Eduardo, a Hispanic 17-year-old musician, leaves his profile visible because 
his music is available there. He hopes others will listen to it and he wants to be able to connect with other musicians and be accessible to potential fans. Some teens eschew the pressure to go private because they think that this implies they have something to hide. For example, Shean, the 17-year-old from Los Angeles, keeps his profile public because, as he says, "I don't believe there's anything on my page that's private." At the same time, he acknowledges that some of his friends have turned theirs private to avoid specific people and to keep spammers away.

Teens who chose to make their profiles private also had specific reasons for wanting to control who could access their content. Some, like Penelope, the 15year-old from Nebraska, wanted to be able to know their audience. Penelope explained that she kept her profile private "so that I know who's looking at my page at what time." While she is not actually aware of who is viewing her profile, she wants to have control over who might be able to. Others, like white 15-year-old Ann from Seattle, make their profiles private as a safety precaution. Ann explains, "My profile is private and friends only because I want to be safe and don't want my parents to worry." For Ann, and for many other teens who joined after hearing various horror stories, visibility is not worth the potential risks. Laura, a white 17 -year-old with Native American roots from Washington, explains, "The risk of having an open profile is not worth it to me. I want to feel in control of my own personal safety. I don't want the whole world to be aware of my feelings or what school I go to." She is concerned that ill-intentioned strangers could use her information against her and she thinks keeping it tightly guarded is a form of protection. Many teens keep their 
profiles private to prevent access by parents, teachers, siblings, college admissions officers, or others who might use their content against them. Others do so in an attempt to avoid spammers, scammers, marketers, and phishers.

Even those who want to be found by some are not keen to be found by all and those who think they have nothing to hide are not necessarily craving to be seen by just anyone. Many of those I interviewed who had adopted MySpace early on often saw the site as teen-centric and saw no need to make their profiles private because being public made connecting with friends and classmates easier. Through time, as adults began to join and other issues emerged, many of these teens made their profiles private. Their stance-"public by default, private when necessary"-is an approach I regularly saw teens take to privacy-related issues. The rise of adults and the potential dangers of unwanted attention forced teens to revert to a more private stance, although they often preferred the easy access that being public allowed.

Being "public" made sense when the relatively homogeneous potential audience did not create conflicts in how people presented themselves, but when teens started facing unwanted audiences, many chose to control access instead of limit selfpresentation.

While most teens I interviewed do not regret moving toward a Friends-only model, it does complicate the process of Friending on MySpace. With public profiles, teens could justify not accepting Friend requests from people whom they knew but did not feel close to. When they made their profiles private, they closed 
off visibility to all but their Friends. Because of this, those who switched from public to private felt more compelled to include their broader peer group among their Friends. Facebook altered this dynamic somewhat, allowing teens to make their profiles visible to all classmates without accepting them as Friends. At the same time, other pressures often motivated teens to include all classmates as Friends. This is discussed in more detail in the next chapter.

The desire teens have to control access also has a counterpart in their desire to know who is viewing their profile. Teens like Penelope, the 15-year-old from Nebraska, limit access in order to know who is viewing her profile, but teens also seek out other mechanisms to keep tabs on their visitors. This desire to know drove software coders to write "trackers" for MySpace, allowing users to copy and paste bits of javascript code into their profiles to capture the IP addresses of those who visited their pages. Some of these initial scripts worked, but MySpace quickly blocked them. This did not reduce their desirability, though, and users began scouring the web for alternatives. While some coders tried to write legitimate software to perform this task, a host of scammers began capitalizing on teens' desire to have this feature. They disguised phishing and spamming code as trackers, getting millions of teens (and adults) who do not understand what their scripts do to install them on their pages. This malware did tremendous damage to MySpace and the company spent extensive time trying to stop what scammers were doing. In 2007, Tom Anderson, the founder of MySpace, posted an entry, pleading with users to not 
add trackers to their profiles. ${ }^{9}$ The company posted bulletins about spammers, phishing, and bogus scripts. Yet as I write this, searching for combinations of "myspace profile tracker" produces millions of hits in Google for sites offering free code to track profile viewers. Some claim to be "official" and others claim to be "the most advanced," but the fact that many of them claim to be "real" is most indicative of the problem that this desire has produced. None of these trackers works, but teens still copy and paste code from these sites into their profiles in an effort to know who is visiting.

On the surface, controlling and tracking access is often viewed as being about safety, but in practice, it ends up serving as a mechanism to bound context. Teens want to have a sense for who is present so that they know what is appropriate. As adults joined the site, teens also wanted to control for what happens when their selfperformances are used against them, either because of misunderstanding or disagreement. Two such incidents forced Kira, a Latina-white 17-year-old in Seattle, to make her profile private and peers-only. First, her grandfather found her public profile, where she had filled out answers to a quiz that asked, "What Sublime song are you?" Her answers resulted in her getting the song "Smoke Two Joints" and her profile displayed some of the lyrics: "I smoke two joints in the morning, I smoke two joints in the afternoon." She liked the song and so she did not think much about it until her grandfather caused everyone in her family to "flip out" by

${ }^{9}$ http://blog.myspace.com/index.cfm?fuseaction=blog.view\&friendID $=6221 \&$ blogID $=236538717$ 
informing them that she was abusing marijuana. After that, she made her profile private but left her stepmother as one of her Friends. Her grandparents later used her stepmother's account to access her profile and misinterpreted a photo she posted of a group of girls with "I love Colleen and Kylie and Brook" below it. She had come out as bisexual to them and they interpreted the profile note as an indication that she was sleeping around. After that uproar, she de-Friended all adults. In recounting this story, Kira expressed frustration that she could not say anything online without adults misinterpreting what she said.

Misinterpretation is one challenge teens face. Clyde, a Hispanic-white 16-yearold from Michigan, keeps his profile private because he is afraid that people might misinterpret the comments left by his Friends and believe that he is a "jerk." His comments include "Screw you over. You suck. Go get a life, [and] just mean comments nobody would get besides me." He knows his Friends are joking around, but he thinks that others might not realize this. Another issue for teens is that their friends and parents do not necessarily share their views on what is appropriate. In Seattle, James, a white 17-year-old with Native American roots, decided to make his profile private after watching a friend get into trouble. After a party, his friend's Friends posted comments on his MySpace page, saying things like, "Oh, man, you were so messed up at that party. You were so drunk. You were so high." His friend's mother saw the comments and promptly grounded him. James was not concerned that his parents would object to his profile, but he was concerned that his Friends might post something that would get him into trouble with his parents. Because 
teens' self-presentations are co-constructed by those around them, controlling access is also desirable to prevent broader audiences from accessing content that they do not control.

Teens rely on privacy features to control the social situation online. These features, while beneficial, are not without their problems. As discussed in the next chapter, choosing whom to Friend can often be socially awkward. Thus, teens are often forced to include people from different social contexts as Friends or to include those with whom they are not that close. Thus, their ability to constrain the audience does not necessarily resolve their desire to have a coherent context in which to act.

\subsection{Managing Identity in Networked Publics}

As teens write themselves into being, couched in the context of their peers, they are forced to face the ways in which impression management in networked publics is unlike that which takes place in face-to-face encounters. The technical affordances for defining the situation and presenting on eself are quite different, forcing teens to explicitly articulate their identity, imagine the context in which they are operating, and negotiate the impressions they are conveying with few structures for feedback. Much of what they face is an extension of what Meyrowitz (1985) described in his discussion of how electronic media alters the negotiation of situations. Yet Meyrowitz, writing before the rise of the Internet, did not account for what would happen as the processes that celebrities and public people faced behind the screen became an everyday part of life. 
Unlike public people seeking to self-define to an unknown audience using electronic media, teens are constructing their presentations of self for people with whom they interact every day. What plays out online influences unmediated situations and what takes place in school shapes the social situations defined in networked publics. For teens, the two are constantly entangled and the context in which they are operating spans both mediated and unmediated encounters. And yet in each environment, teens must contend with other forces. In school, teens are faced with adults who seek to control the social situation. In networked publics, they must face invisible and unknown audiences, many of whom hold power over them. Their efforts to define the situation and control the context are often thwarted by adults who seek to control peer worlds.

In all environments, teens' identity is often framed in relation to those around them. The social categories that shape social relations in school (Eckert 1989) help define teens' identity, both in the ways they themselves identify with those social categories and in the ways others mark them. Online, this is made more explicit. Social relations are publicly articulated and teens' profiles comprise not just what they themselves explicitly state, but also what others state about and to them. Goffman (1959) speaks of how a person's presentation of self comprises what they explicitly give and what they give off. While much of what teens explicitly include in their profiles gives off important signals, what their Friends say gives off signals at a whole new level. 
More than anything, what makes impression management different in mediated and unmediated environments is the ways in which acts of self-presentation do and do not iterate. Often, it is impossible for the performers to assess the success of their presentations in conveying their sense of self to the audience. There are no feedback loops that allow teens to adjust based on real audience feedback and, often, there is no way of truly knowing who the actual audience is. Even a teen's intended audience might not actually be consuming the content produced and the actual audience might be quite different from the perceived or imagined one. Given the multiple environments in which teens operate with the same people, feedback does occur, but changing one's profile is not an ephemeral act and these adjustments might come long after damage is done.

While teens understand the context in which they are operating, they may not always understand the context in which their friends are operating. In unmediated environments, witnesses can look around and gauge the audience themselves, but online, this is not possible. Thus, when teens contribute to their friends' selfpresentations, they may put their friends in uncomfortable positions when their ideas of the context are not compatible. Teens' lists of Friends help convey what the teens intend and imagine to be their audience, but they may not help resolve the conflicts that teens face. As core parts of profiles, these lists allow teens to express information about their identities, but they also feed directly into the ways teens negotiate status and maneuver in peer worlds. 


\section{Chapter 5: Friendship, Status, and Peer Worlds}

When Skyler Sierra's mother asked her to explain why MySpace was so important, the 18-year-old from Colorado turned to her mother and said, "If you're not on MySpace, you don't exist." For many of the teens whom I met, participating on social network sites is a necessary part of participation in peer culture. Social network sites are one of the many forms of social media that fill in social gaps by allowing teens to connect when getting together is not possible. While teens may see each other at school, in formal or structured activities, or at each other's houses, social network sites support a much more unstructured environment for peers to gather more broadly. Teens use networked publics to support peer socialization. In doing so, many re-create many of the complex social dynamics that play out wherever teens gather. At the same time, technology introduces new structural forces that alter the social dynamics.

Teen practices involving social network sites mirror many of those that scholars have documented in other places where teens gather with peers (Eckert 1989; Milner 2004; Skelton and Valentine 1998). Just as they have done in parking lots and shopping malls, teens gather in networked publics to negotiate identity, gossip, support one another, jockey for status, collaborate, share information, flirt, joke, and goof around. In other words, they go to social network sites to "hang out." Through mediated interactions, social network sites allow teens to extend their social worlds beyond physical boundaries. Conversations that begin in person do not end when 
friends are separated. Teens complement private communication through messaging and mobile phones with social network sites that support broader peer engagement.

As with other forms of social media, most teens do not treat social network sites as alternative or "virtual” worlds (Osgerby 2004; Osgerby, Abbott 1998). Social network sites are simply another place for teens to connect with their friends and peers; participation fits seamlessly into their everyday lives and complements other practices. Just as they use more intimate channels of communication to maintain "full-time intimate communities" (Matsuda 2005) with close friends, teens use social network sites to build "always-on" networked publics inhabited by their peers. Teens will usually have a small circle of intimate friends with whom they communicate in an always-on mode via mobile phones and IM, and a larger peer group that they are connected to via social network sites. Social network sites are one of many types of social media that teens use to engage with friends and peers, but they were the most dominant type of networked public that teens used during my fieldwork.

Social network sites are replacing physical gathering spaces for youth out of necessity. In the 1980s, the mall served as a key site for teen sociability in the United States (Ortiz 1994), because it was often the only accessible public space where teens could go to hang out (Lewis 1990). Teens are increasingly monitored and many have been pressured out of public spaces such as streets, parks, malls, and libraries (Buckingham 2000; Valentine 2004). Lacking other options, U.S. teens 
have turned networked publics into their contemporary stomping ground. Just as teens flocked to the malls because of societal restrictions, many of today's teens are choosing to gather with friends online because of a variety of social and cultural limitations (boyd 2007). While the site for gathering has changed through time, many of the core practices have stayed the same. What differences exist stem from the ways in which social network sites alter common social practices.

The relations and dynamics that play out in school extend into the spaces created through networked publics. What takes place online is reproduced and discussed offline (Leander and McKim 2003). When teens are socializing, online and offline are not separate worlds-they are simply different places to gather with friends and peers. Conversations may begin in one environment, but they move seamlessly across media so long as the people remain the same. Social network sites mirror, magnify, and extend everyday social worlds. At the same time, networked publics provide opportunities for always-on access to peer communication, new kinds of social pressures, public display of connectedness, and unprecedented access to information about others. As a result, technology inflects different aspects of teen sociality and peer social structures.

In this chapter, I examine how social media shapes peer worlds, focusing primarily on how social network sites are leveraged in and alter teen sociality. I center my analysis on practices that are salient in teens' lives, like friendship development and maintenance, and core structural dynamics like social categories. 
My goal is to investigate the role social network sites play in the lives of today's teens by considering what has changed and what has not. I found that the majority of mediated teen practices replicate everyday interactions and dynamics, but that technology inflects these dynamics in critical ways, often by magnifying and formalizing interactions, conflicts, and social divisions.

\subsection{Peer Relations and Teen Friendship}

Teen friendship practices in social network sites and networked publics need to be understood in relation to the broader contexts of teen sociality as it plays out in U.S. high schools. For contemporary teens, the age-segregated institutions of school, after-school activities and sports, and youth-oriented consumer culture continue to be strong structuring influences. The peer relations of teens are structured by adult society and educational institutions organized by rigid age boundaries. There is a cultural consensus that the ability to socialize with peers and make friends is a core part of growing up (Berndt 1996; Newcomb and Bagwell 1996). Academics are only one part of why youth are sent to school. Youth are also put in educational systems and immersed in peer groups to learn how to develop friendships (Corsaro 1985; Howes 1996). In theory, the "personal communities" that children and teens develop help them negotiate identity and intimacy (Pahl 2000). At the same time, the groups that form-and the social categories that define them-can reinforce gender, race, and class divisions. 
The teen years are marked by a rise in peer-centered sociability as adult oversight recedes and status and popularity battles that we typically associate with middle school and high school take hold. This is also the period when performances of sexuality—primarily heterosexuality—emerge and relationships marked by sexual attraction begin to complement same-sex friend groups (Eckert 1996; Pascoe 2007; Thorne 1993). These everyday dynamics of teen life are replicated and reinforced online.

Social categories also play a significant role in structuring the social worlds teens inhabit (Cotterell 1996; Eckert 1989; Milner 2004). Labels like “jocks and burnouts” (Eckert 1989), “nerds and normals” (Kinney 1993), and "freaks, geeks and cool kids" (Milner 2004) identify different social categories. Each label is associated with stereotypes about tastes, practices, and attitudes. Peer status is inferred through these social categories. Teens struggle to position themselves in relation to social categories and, in doing so, reinforce them. Pop culture also reinforces social categories through its portrayal of cliques and peer status. From Grease to Mean Girls, Hollywood uses social categories as a prominent plot props in films about teenagers.

The social categories that exist in most high schools today are not nearly as simplistic as those on the silver screen. In trying to describe the structural dynamics that they experience at school, teens struggle to account for the ways in which their experiences do and do not match up to the images presented by the media. Jessica, a 
white 14-year-old from Massachusetts, rejected the notion that her school has cliques because, while there are distinct groups of friends, "it's not like completely separate." In Jessica's mind, a clique is a group of friends who refuse to interact with anyone else. Her school is a small charter school and while there are distinct friend groups, she does not see these as cliques because there are no clear boundaries. Sara, a white 16-year-old from Austin, Texas, also rejects the notion of cliques because, to her, the term "clique" implies labels and hierarchies. Yet Sara's notion of a friend group resembles Jessica's idea of a clique: "people who hang out with each other, and not anyone else." Two of her classmates-Catalina, a white 15-year-old, and Jordan, a Mexican-white 15-year-old-concur. They note that friend groups in their school are very tight and that people would feel uncomfortable sitting with friend groups other than their own at lunch.

While teens struggle with the term "clique," most acknowledge that friend groups play a dominant role in structuring their social worlds. When I asked teens how friend groups emerge, they pointed to similar tastes, activities, and identity markers. For example, Court, a white 17-year-old from Nebraska, told me that friend groups emerge when "the style is the same, you listen to the same music, you hang out with the same people." Teens accept that "birds of a feather stick together," but they are not always aware of the structural forces that drive such homophily (McPherson et al. 2001) or the role that it plays in shaping the social groups that they associate themselves with. 
Friend groups define peer worlds. Yet these friend groups are not haphazardly determined-larger structural forces shape them. Social categories emerge from and help configure friend groups. As documented by Penny Eckert (1989), social categories are driven by social conditions, primarily class. The social categories of "jocks" and "burnouts" are loaded with class-marked references and the structural conditions of high school reinforce class distinctions through the valuation of jocks and demonization of burnouts. While the labels have changed, it is clear that these same divisions are still operating in the schools of many of the teens I interviewed. The social categories are visible through social networks of teens and the geographies they inhabit. They are also displayed and reinforced online.

The underlying dynamics of teen friendship and peer relations have not changed. Neither school-driven friendships nor social categories are new. What has changed is where these dynamics play out. Rather than gathering at malls or in parking lots, teens are leveraging social media and connecting through social network sites. The same pressures to fit in and stand out extend to these environments and these environments mirror and magnify the dynamics underlying teen friendship and peer relations.

\subsection{Pressure to Participate: Signing Up and Opting Out}

Teens typically join social network sites because their friends are all using them and they feel the need to participate. The ability to maintain and enrich social connections, keep up with gossip, and gather with friends when in-person 
encounters are impossible all drive participation. Social network sites allow teens to interact with their broader peer group, including fellow classmates whom they do not know particularly well and members from neighboring schools whom they might see at sports events. This allows teens to interact with and get to know peers with whom they are only acquainted. For example, white 15-year-old Melanie from Kansas values Facebook because it "makes it easier to talk to people at school that you may not see a lot or know very well. Just to say something quick." Melanie sees Facebook as a fantastic tool for building new friendships with people from school. Aaron, a white 15-year-old from suburban Texas, likes that he can use MySpace to get in touch with classmates even when he does not have their phone numbers. This is valuable for getting homework assignments as well as arranging times to connect in person. In other words, both Melanie and Aaron use social network sites to develop and maintain broader peer connections. While teens often have close friends, their connections to their broader peer group vary tremendously.

Despite the perception that social media is enabling teens to reach out to a new set of people online, the majority of teens define their peers and friendships by the relations fostered in school. Social network sites theoretically enable teens to connect to new people (Rheingold 1994), but this is not the dominant paradigm. Surveys of U.S. teens indicate that most teens use social media to socialize with people whom they already know or with whom they are already loosely connected (Lenhart and Madden 2007a; Subrahmanyam and Greenfield 2008). Most of the teens I interviewed underscored that those they knew online were the same as those 
they knew from school. For example, Sabrina, a white 14-year-old from suburban Texas, explained that while she uses MySpace, she never uses it to meet new people. "I just find my friends and hang out." The dominant and normative social media usage pattern is to connect with friends, family, and acquaintances, thereby reinforcing the structural dimensions of peer social worlds that exist in schools. It is less common for teens to seek out new friends online; doing so can sometimes be stigmatized because of prevalent stranger danger rhetoric.

Many teens believe participation is essential for keeping up with peers at school. Sitting in a coffee shop in suburban Michigan, I asked Tara, a Vietnamese 16-yearold, about Facebook. She giggled and told me that she had "an addiction" to the site. She logged in regularly to check for new messages from friends, read updates about her classmates, and commented on friends' photos. I asked her why she joined the site and she said, "Like everyone says get a Facebook. You need to get one." Tara thought joining was a social imperative. Initially, when her friends started joining, she tried to resist out of concern that Facebook would take up too much time. Yet as more friends joined and became engaged with the site, Tara decided that she had to join.

In his examination of high-school social structures, Milner (2004: 4) suggests that teens' obsession with status exists because "they have so little real economic or political power". He argues that hanging out, dating, and mobilizing tokens of popular culture all play a central role in the development and maintenance of peer 
status. Working out markers of cool in the context of friendship and peer worlds is one of the key ways that teens do gender, race, class, and sexuality work (Bettie 2003; Pascoe 2007; Perry 2002; Thorne 1993). The struggle to fit in and keep up is a salient force in teen life, especially for girls (Eder et al. 1995; Milner 2004). Many of teens' choices, from which clothes to wear to which hobbies to adopt, are driven by their desire to be part of the crowd. Teens flock to social network sites because these sites represent an arena to play out these status negotiations beyond the school yard and living room. Using these systems, teens struggle to fit in, stand out, and keep up with what is cool. Social pressures shape both participation and nonparticipation. Because these systems create always-on peer groups, participation and mediated maintenance of social relations_-not simply presence in physical space-structure many aspects of teens' social worlds.

\subsubsection{Pressure to Join}

When I met Milo, an Egyptian 15-year-old from Los Angeles, he detailed how his friend got him into MySpace:

"He was like, 'Yo bro, you need to get into MySpace.' 'What are you talking about?' He was like, 'Yeah, MySpace is the thing now.' It's like, 'All right,' signed up. He first did my page. I was like, 'Hey, how do you do that?' He was like, 'Here, go to this.' I was like, 'All right.' I was like, 'Hey, this is wack,' and I got into it." 
The thought process that led to Milo's joining MySpace is quite similar for that of many teenagers whom I met-friends encouraged participation by marking MySpace as the cool thing and, often, teens helped their friends and peers take the first steps toward joining.

Most teenagers join social network sites to connect with friends and peers, but there is also social pressure to do so. On one hand, teens directly pressure peers to join. On the other, teens feel left out if they do not. For Sabrina, the 14-year-old from Texas, pressure came in the form of a friend: "My friend Christian said, 'Get a MySpace now!' Okay. So I just kind of got one." She had been concerned about the potential dangers of the site, but Christian convinced her otherwise. She felt more reassured about the site when she realized many of her classmates and friends were also there. Other teens joined because of more collective peer encouragement. For example, in Los Angeles, Zero, a black 15-year-old with Native American roots, told me that he created a profile because "at the time, everybody was like, 'Do one. Join it.' I'm like, 'Okay, what the heck?'” For Zero, it was not pressure from one person but ongoing encouragement from his peers more generally.

Like Sabrina, Lolo, a Guatemalan 15-year-old from Los Angeles, had also hesitated out of fear. When she found out a close friend had joined, she got upset and asked, "Why do you have one of those? There are like rapists or whatever." She tried to convince her that MySpace was dangerous, but "then everyone started getting one and then I got one." While no one had convinced her that the site was 
not dangerous, she was more concerned that she was missing out. Lolo is not the only teen who joined out of fear of being socially disconnected. Some teens like Lolo worry about keeping up with what is happening with their friends; others worry about being left out of what is cool at school. Penelope, a white 15-year-old from Nebraska, joined MySpace because people at school were talking about it and she "felt out of the loop." Being able to gain access to school gossip and the dramas of the "popular" teens gave Penelope a sense that she was better connected at school. MySpace gave her a window into her classmates' lives, but Penelope's ability to follow along did not make her a part of their social circuits.

Teens have long consumed in an effort to gain peer status (Milner 2004). Indeed, some teens can-and do-achieve some level of status when they gain access to valuable material goods that are difficult to find, particularly when the desired object can be shared. Attaining a rare pair of Nikes might have been a valuable status marker, but having acquired a Wii gaming system while they were hard to come by could prompt a social invitation in the hopes that the Wii would come along. Both the Nike and Wii are valuable markers of cultural capital-they are generally valued but hard to attain.

Social network sites are slightly different. Being an early adopter of MySpace and Facebook was a marker of cool in certain circles. The capital that teens attained by being early adopters was more "subcultural capital" (Thornton 1996) than cultural capital. The value was about being "in the know" rather than having access 
to something rare but generally valued. In this way, being an early adopter of MySpace or Facebook was akin to being at a cool underground rave in that subculture, where simply knowing the location of the venue denoted status. Joining MySpace or Facebook once it became popular was necessary for baseline social membership, but it did not in itself afford status. Still, many teens who struggle to fit in joined social network sites in the hopes that participation alone would elevate their status. In this way, they conflated cultural participation with social membership.

Initially, social network sites grew popular through word of mouth (boyd and Ellison 2007). Teens convinced their friends to sign up; they, in turn, convinced their friends. Schools served as local clusters and, in most cases, a handful of teens would learn about a particular site and popularize it inside their school. While high school is still the primary social sphere for U.S. teens, teens also have friends from extracurricular activities, church, and summer camp; cousins and friends who moved away extend their social networks further. It is through these extended networks that social network site knowledge passed from school to school. Eventually, as mainstream media became familiar with teen adoption, it further enabled the spread of the phenomenon.

Social network sites, like other genres of social media, are most valuable with high network density. In other words, teens gain the most value from social network sites when all of their friends are also using the site. Network density is important 
for the various communication features to have value, but, thanks to the Friends feature, it is also significant for identity display. Not only do teens want to be able to interact with their friends on the site, but they also want to be able to list those friends as Friends as a public display of status through connections (Donath and boyd 2004). MySpace's Top Friends feature further amplifies this because teens want to make sure that certain people are listed there.

Many teens in my study felt compelled to get their friends to join, either to achieve network density or to make certain that said friends were included in conversations and representations. When those friends refused or were too lazy to join, teens sometimes opted to make an account for them. The practice of making accounts for others is mainly confined to MySpace, although it does also happen to a lesser degree on Facebook. Michael, a white 17-year-old from Seattle, was unfamiliar with MySpace until his girlfriend introduced him to it. After he failed to make an account for himself, his girlfriend decided to do it for him. She wanted to list him in her Top Friends. While he did not create the account, he got into MySpace after he had an account created for him. Keke, on the other hand, had an account created for her but she never got into the site. A black 16-year-old from Los Angeles, Keke had no interest in MySpace because she's "not a computer person." All of her friends use MySpace and, after realizing she would never make her own account, they decided to make one for her. She knows she has this account and her friends showed her the profile, but she has never gotten on it. Instead, her friends check her profile and tell her if anything happens. 
When teens make profiles for their friends, they typically do more than create an account. They often fill out the profile information to the best of their ability, choose a background and design the layout of the page, upload photos of their friend, and, in some cases, respond to their comments and messages for them. In other words, these teens take on their friend's identity to create a digital representation of the person. While some teens never engage with the profile that their friends create for them, most get curious and engage. Others have their friends manage their profiles because of structural limitations. For example, Mickey, a Mexican 15-year-old from Los Angeles, is able to access MySpace only through his phone because his parents restrict his accessing the site. Through his phone, he can do only certain things so, as he explains, "I just gave my password to my friend and my friend like pimps it out." Mickey's friend updates his profile and tells him about changes that he cannot see via his phone.

While creating and managing someone else's profile is rare, teens often create their profiles while in the presence of friends who already participate. Collaboration is especially enjoyable for teens who are uncertain about how to create a meaningful self-representation or those who lack the technical knowledge to alter the background and layout. In explaining how his profile was created, Shean, a black 17year-old from Los Angeles, shows how the collaborative process results in a tremendous learning opportunity: 
"I was at one of my best friend's house and she was like, 'Have you heard of this thing called MySpace? It's so cool, you should get one,' and she showed me her whole web layout and she knew how to make your page and redesign it and all that. It took us all day to redo my whole page and do pictures and all that-she taught me how to do that."

Sitting alongside a friend with more expertise, Shean learned how to navigate MySpace to design an elaborate profile. Not only did Shean join MySpace because of his friends, but he also learned how to navigate the technical landscape. Learning how to manipulate MySpace code is both practical and a form of media literacy (Perkel 2006). While teens like Shean often join to socialize with friends, the process of developing a profile can lead to new interests and the development of technical knowledge.

\subsubsection{Failure to Engage}

While participation is valued by most communities of teens, not everyone participates. There is no current accurate data on how many teens have never created an account or how many have deleted their account, but it is clear that not all teens do participate. In every community I visited, I met or heard about teens who did not participate, although I never met a teen who had not heard of either MySpace or Facebook. Discussion of nonparticipation is important because not participating when participation is normative can alter teens' relationships with their peers. 
The reasons behind nonparticipation vary, but three categories of nonparticipants appeared to dominate. They are disenfranchised teens, conscientious objectors, and former users. Disenfranchised teens lack access either because of structural limitations or social restrictions. Conscientious objectors choose not to participate, although their choice is frequently correlated with other sociostructural factors. Increasingly, there is also a third category of former users who once participated and no longer do. Many of these teens never did more than create a profile. For example, Kaleb, a black 15-year-old from Michigan, says that social network sites are not popular in his friend group. His friends had tried it, but they found it boring and so they deleted their accounts; he kept his. Other former users were once active participants but deleted their accounts because of circumstances. For example, Anindita, an Indian 17-year-old from Los Angeles, was active on MySpace until her boyfriend demanded that she delete her account; she made a new one as soon as they broke up. Lolo, the 15-year-old from Los Angeles, told me that it is common for people to become nonusers when they are in relationships, as she had done. The reasons that most former users delete their accounts stem from the same roots as those that prompt some teens to never participate at all. For this reason, I focus on what underlies disenfranchisement and conscientious objection.

Two factors drive disenfranchisement: social restrictions and structural limitations. The most common form of social restriction is that imposed by parents, although parents are not the only restrictors. Some private schools threaten 
expulsion if they find that their students have profiles. Some teens have significant others who threaten to end the relationship if an account is created or not deleted. While teens who are forbidden from creating accounts can-and sometimes choose to-find workarounds, the assertion of power by parents, schools, and significant others is still a form of disenfranchisement. It forces teens to sneak around to avoid getting punished.

The most dominant structural limitations teens faced concerned issues of access. On one hand, some teens simply have no Internet access anywhere. This is typically understood as the "digital divide" (Katz and Aspden 1997). While such teens do exist, typically in poorer communities, the access divide has nearly closed for American teens (Lenhart et al. 2005). Yet not all access is created equal. Many argue that the digital divide is not simply a matter of access but of technical skills, media literacy, and quality of access (Hargittai 2002; Martin 2003; van Dijk and Hacker 2003). Schools and libraries invest heavily in filtering software to limit teen access to problematic content. The result is that MySpace and Facebook are often banned in the public places where poorer teens gain Internet access. Filters are also used in homes by parents who wish to enforce social restrictions through technical means. Such filtered access creates a new divide because teens with limited, filtered, and heavily regulated Internet access do not have the same opportunities and learning potential as those who can access the Internet freely at home. Examining the Internet practices of rural teens, Mary Gray (Forthcoming) found that MySpace participation is virtually nonexistent in rural Kentucky. While many of the teens she 
met had Internet access through schools and libraries, all of these institutions blocked MySpace. While teens in such environments do not feel local pressure from their peers to participate, they are written out of broader cultural trends and lack the sociotechnical familiarity to engage with teens from more media-rich environments. They are part of a growing "participation gap" (Jenkins et al. 2006) that separates teens not based simply on access but on quality of access.

Filtered access can be circumvented through technical means. Many of the teens I met in urban settings were familiar with proxies. Proxies allow teens to visit an unblocked site that will serve as a go-between and provide access to blocked content. Systems administrators can—and do-block each proxy site that they learn about, but new ones keep popping up. Thus, teens in such environments traffic in knowledge about which proxies work and which are already blocked. Ty, a black 17year-old in Los Angeles, does not have access to the Internet in the group home in which he lives. He relies on school access, but his school blocks MySpace. His use of proxies exemplifies the circumvention mechanisms that some teens employ to gain access:

"[My school tries] to block [MySpace] so bad but it just doesn't work. You always find a way to get around it. If I'm using a certain proxy that I have and like the next day I try to use and it's blocked, I'm like, 'Dang, it's blocked.' But then I'll go around and I'll look at like on another person's computer or whatever and I'll see they're on MySpace and I'm like 'Oh, how you get on, man, come on.' And 
they'll share with me the proxy that they use and then I'll get back on. So it just keeps on going around in a big old cycle."

Proxies are a central form of technical currency in some communities, while teens in other communities do not know that they exist. Familiarity with proxies appeared to increase in communities with low levels of home access, especially in more urban settings. Teens learned of proxies through friends and older siblings, suggesting the existence of grounded knowledge networks through which information flows. While proxies work equally well on home filters, I did not run across teens who used this approach to circumvent parental restrictions; visiting a friend's house appeared to be more common.

Teens who are truly disenfranchised are more often than not restricted on both social and structural levels. One such example is Egyptian 15-year-old Mic, the only son of an immigrant family living in Los Angeles. Initially, Mic wanted to get on MySpace because it was popular among his classmates, but he was forbidden. MySpace is not his only restriction. Mic does not have Internet access at home because "Dad always hears bad things happening on MySpace and he doesn't think I'm mature enough to get the Internet at this point." His school bans all social network sites; while Mic knows about proxies, Mic is afraid that if he were caught at school, his father would find out. Even if Mic wanted to try out a proxy and visit a social network site, his time at the school computer lab is extremely limited. Mic's father drops him off at school right before the first bell, removes him from school 
during lunch, and picks him up immediately after school. He is not allowed to be involved in school activities and so his only Internet access is during class time. Mic has few options for Internet access or, for that matter, social contact. His friends are not allowed to come to his house and it is very rare that he can go over to someone else's house; his only regular social time with peers is during Sunday school at church. His cell phone is prepaid and he is allowed to use it only to contact his parents from school. Mic says that his father does not trust him to control himself when faced with temptations. While he cannot use the Internet at home, he does have limited access to TV and is especially fond of Lost. When he gets free time at school, he uses the Internet to learn more about the show's plot. While Mic does not like the restrictions he is faced with, he accepts them without question.

Mic's acceptance of his situation has multiple layers. At one level, Mic believes that this is simply part of being from an immigrant family. Given Mic's depiction of his social world, there is also a decent possibility that Mic's father fears what assimilation might mean. At another level, Mic feels a pressure to live up to his father's expectations. Mic knows his father immigrated to give him opportunities and he feels tremendous pressure to focus on schoolwork. To resolve the conflicting narratives at home and school, Mic frames his nonuse to be a matter of personal choice. While he initially wanted a profile and all of his friends have profiles, he now believes that MySpace is dangerous and that his father was justified in not wanting him to be on the site. Although his nonparticipation stems from issues of 
disenfranchisement, he is able to accept his lot by reframing it as a matter of personal choice.

In addition to the disenfranchised, some teens choose not to adopt social network sites. Most conscientious objectors fall into four categories: teens who are too busy to participate, teens who are concerned about their safety, marginalized teens who think that social network sites are for the cool kids, and teens who, according to their peers, think they are too cool for these sites. The latter two groups frequently justify their nonuse as "because it's stupid." With MySpace, I found a few politically minded teens who opposed Rupert Murdoch (and, thus, MySpace) and some religious teens who thought that MySpace was a sinful environment, although these two explanations were more common among adults.

When teens referenced time, they typically emphasized that they were too busy with school, activities, and work. Some included a rich social life in the list of things that made them too busy, but this was less common. Myra, a white 15-year-old from Iowa, laments her schedule, constructed mostly by her well-meaning mother. Guessing that 98 percent of her time is taken up by scheduled or structured activities, she says she simply does not have time for Facebook or MySpace. Although Samantha, a white 18-year-old from Seattle, uses MySpace, she says, "People who have lives are not using them because they have better things to do. The people who have sports to do and jobs to go to and friends they hang out with a lot are the people who I think don't use them as much." Overscheduling of teens is a 
relatively recent phenomenon, although it is rooted in a broader frame of concerns about unstructured leisure time. As child labor disappeared in the early twentieth century, youth spent more time in leisure activities, primarily those involving social interaction (Larson 2001; Wartella and Mazzarella 1990). Early fears contributed to the rise of compulsory schooling while ongoing concerns contributed to the rise of organized and structured activities. As research showed that unstructured time resulted in delinquency (Osgood and Anderson 2004) and that learning took place in organized activities (Mahoney et al. 2005), many parents began to value structured and organized time. In my fieldwork, I found that many teens from wealthier and more educated families had little unstructured time in their daily lives. While organized activities may have benefits, the overstructuring of teens' lives left them craving opportunities to interact with peers in less controlled settings.

Fear is another common explanation for nonparticipation. Some teens fear potential stalkers while others are concerned about their reputations. Sabrina, the 14year-old from Texas, saw her initial nonparticipation as rare. When her teacher asked who did not have a MySpace, only she and one other classmate raised their hands. In explaining why, she told the teacher, "I don't want to get stalked and die." She told me that the other classmate shared her concern. While fear prevented her from joining for years, she did eventually join after a friend convinced her that her fears were unreasonable. While Sabrina's nonuse primarily stems from her own fears, other teens conscientiously reject social network sites out of respect for their parents' fears. Fear of sexual predators is the dominant explanation for fear-driven 
nonparticipation, but some teens fear how participation might affect their futures. In Kansas, a white 16-year-old named Lilly told me that one of her classmates was afraid to join Facebook out of fear that her reputation would be blemished: "She's afraid, because people could put a picture of you Photoshopped next to a beer as a joke. Not that she's the kind of person that anybody would do that for, but she's the kind of person that would worry about somebody doing that."

While Lilly's classmate chooses not to participate to keep her reputation from tarnishing, other teens worry that they do not have enough of a social standing to participate. Teens who lack strong social connections with others using the sites often resist joining out of concern that participation would be more socially awkward and embarrassing than enjoyable. Often, this explanation is confounded with lack of technical familiarity. For example, Fred, a white 15-year-old from suburban Texas, opted not to join MySpace because "I don't know it as well-I don't know what to do-I don't know that many people on it." It is clear in our conversation that while Fred has classmates on MySpace, none of his close friends use the site. The combined lack of technical and social familiarity is a significant barrier for Fred. Other teens are more concerned that they lack the social connections to create a profile that is not embarrassing. For them, it is better not to have a profile than to have one without any Friends.

Finally, there are teens who supposedly think they are too cool for social network sites. Teens never gave this explanation for their own general nonuse, but 
this explanation regularly emerged as teens tried to explain why some of their friends and peers opted out. Gabriella, a Honduran 15-year-old in Los Angeles, says that her boyfriend does not have a MySpace because "he says it's dumb. He says it's immature for him." She rolls her eyes as she tells me this. By mocking his view that he is too cool for MySpace, Gabriella seeks to reject her boyfriend's implication that she and her friends are engaged in an immature activity.

Nonconformity plays a central role in teens' views that others do not participate because they are "too cool." Some teens choose to rebel against popular culture by not participating in whatever is popular. Kevin, a white 15-year-old from Seattle, explains this view:

"There are a lot of people-like how I used to be-that kind of still don't want to [join MySpace] because they want to go against the grain and they don't want to be like everyone else, so I have a lot of friends that aren't on MySpace just because they don't want to be part of that, and because it's culture."

Nonconformity has a long history among teens and is one cornerstone of subcultural participation (Gelder and Thornton 1997; Hebdige 1979). While youth subcultures are frequently viewed as being a rejection of adult society (Cohen 1972; Hall and Jefferson 1976), they are just as likely to be about a resistance to peer culture hegemony (Hodkinson 2002; Leblanc 1999; Thornton 1996). The social categories that emerge in high school can also be understood through conformity and nonconformity to hegemonic views (Eckert 1989). Not surprisingly, some teens 
reject social network sites as part of rejecting dominant peer culture. As participation became mainstream, nonparticipation became a way of rejecting the mainstream norms and values. In turn, those who were engaged dismissed the nonconformists.

As social network sites gained mass popularity, teens were expected to participate. While a plethora of reasons explain why some teens did not, this put them at social risk. Nonparticipation was stigmatized and as Dom, a black 16-yearold male from Washington, explained, "People not using them are known as 'squares."' Teens responded to this stigma in various ways. Some were ashamed that they lacked the access or social standing for their participation to be viable. Those whose access was restricted blamed the social or structural reasons. Finally, in defiance of such stigmas, teens who had opted out sometimes chose to reframe nonuse as cool.

Reframing is exemplified by Sara, the 16-year-old from Austin, who is forbidden from having a Facebook account because her "mom doesn't like all that stuff, 'cause like you hear about all of the sex predators and stuff like that. So, she won't let me have one." There are no restrictions on her computer and her friends are welcome to use Facebook at her house; she is simply forbidden to have a profile. Sara is aware that she could disobey her mother's rule, as many of her friends do, but she chooses not to. Given the other rules that she follows, it is likely that Sara is too afraid of the consequences of disobedience. Initially, she wanted a profile "cause everyone else 
had one," but she no longer cares because, as she explains, "My friends are gonna like me just as much if I do or don't." This confidence in her social standing allows her to reject the importance of having access. She is not afraid that she will miss out because "if there's something that important that I have to see, then [my friends will] show me." Instead of feeling ashamed or angry, Sara argues that not having a Facebook gives her more time to focus on what is important.

Shame, blame, and defiance are all common teen responses to social conditions that place them outside of traditional systems of peer status. Participation and nonparticipation are influenced by and configure the social structures of teen social worlds. When adoption becomes normative in a particular social setting, participation is necessary to being a part of those peer worlds. Nonparticipation does not necessarily result in social isolation, but in some environments, it does mean limited access to peer culture. Participation alone does not confer social status within peer culture, but nonparticipation can put teens at a social disadvantage.

\subsection{MySpace vs. Facebook: Social Categories and Networked Turf}

Social network site participation is not about the technology itself but about the peer groups that connect through a particular technology. As a result, it is not only important to join a social network site but to join the right social network site.

When I began my fieldwork, MySpace or not MySpace was the social network site decision. There were other social network sites, but none had enough traction 
among teens to warrant much consideration by anyone but early adopters. This dynamic changed in 2006 when Facebook emerged as a serious competitor to MySpace for the attention and loyalty of American teens. Thus, during the 20062007 school year, the social network site question changed to "MySpace or Facebook?"

As teen presence on Facebook became noticeable, journalists began speculating that MySpace was dead; they assumed that teens were switching to Facebook from MySpace (Levy 2007). In the field, I saw a far more complex picture. Teens were not simply switching. Teens were using one or the other, both, or neither. MySpace continued to rise in popularity among some groups while Facebook gained traction in others. When asked, teens primarily framed their choice around the presence of friends, specific features or aesthetics, or their understanding of safety. Yet their explanations were laced with value judgments and references to structural conditions. In examining these, I found that "preference" was primarily a reflection of broader teen social structures. In other words, the distinctions in MySpace and Facebook membership revealed the ways in which teen social worlds are divided by social categories, which, in turn, are driven by socioeconomic class, peer status, and other structural conditions.

\subsubsection{Adoption Practices}

Collective action drove Facebook adoption, as it had MySpace, but many of those who flocked to Facebook had never fully engaged with MySpace. In some 
communities, teens had only recently heard of MySpace. In others, there were enough nonusers such that peer density had not yet formed on MySpace. Many of those who drove initial Facebook adoption had never been that involved in MySpace. Teens who were deeply engaged in MySpace felt no need to switch, particularly when all of their friends were already on MySpace. As Kevin, the 15year-old from Seattle, explained, "I'm not big on Facebook; I'm a MySpace guy. I have a Facebook and I have some friends on it, but most of my friends don't check it that often so I don't check it that often. I mostly just communicate with my friends by MySpace because it's efficient." For Kevin, Facebook had no appeal because it was not where he could find his friends. Collective action had driven his friends' adoption of MySpace, but there was no drive to shift to a different site. Collective inertia shaped his friends' choice.

MySpace entrenchment was not universal. Entrenchment was partially shaped by geography, the origins of MySpace, and the viral way in which it spread (see Chapter 3). Because of this, MySpace initially gained traction among teens in urban and West Coast communities before spreading to other parts of the country. I found that outside of the West Coast, many teens in suburban, small-town, or rural environments had not heard of MySpace before the media-driven "technopanics" (Marwick 2008) began in 2005. The moral panic that emerged shaped both use and nonuse. On one hand, there were parents and teens who rejected MySpace because of perceived dangers. On the other, there were teens who rejected what they perceived as adult fear mongering; some ignored adult concerns while others 
embraced MySpace out of rebellion. Because network effects shaped how MySpace was adopted, individual views and decisions influenced collective engagement. Thus, teens tended to share their friends' attitudes toward the dangers of MySpace. Those who failed to engage with MySpace were far more inclined toward an alternative than those who were entrenched.

Some teens who were deeply engaged in MySpace adopted Facebook, but most commonly as an addition to a long line of social media tools they used. Summer, a white 15-year-old in Michigan, called such teens "the designer class." In her school, the teens who flocked to Facebook right away were still using MySpace, Xanga, IM, and other sites. As she explained, "Anything that you can think of, they have it. So it's just kind of like the latest thing." They had not rejected MySpace-just as they had not let go of beloved clothes bought the previous season-but they relished using the new as well as the old.

While some teens joined Facebook simply because it was the next cool thing, others joined because they knew people who were already deeply engaged there. Teens who had friends in college were driven to the site both because of the possibilities of friend maintenance and because socializing with older friends had great appeal. Those who moved or changed friend groups and found that their new peer group was already engaged on Facebook were equally inclined to join. Midway through high school, Red, a white 17-year-old from Iowa, stopped hanging out with the crowd she had been friends with for years and developed friendships with a new 
crowd at school. The new crowd preferred Facebook so she switched her attention there, although she did not bother deleting her MySpace. "I am on Facebook and MySpace. I don't talk to people on MySpace anymore ... the only reason I still have my MySpace is because my brother's on there." While she no longer maintained ties to the friends whom she used to socialize with on MySpace, her brother was important enough to her for her to continue maintaining her MySpace account.

Some teens had truly switched from MySpace to Facebook, but they were rare; switching was far more common as a rite of passage between high school and college. In those cases, switching was about letting go of high school past and moving on to college future. Yet more often than not, when teens joined Facebook, they either never had an account on MySpace or joined Facebook in addition to MySpace.

As distinctions formed, teens began to judge each other based on which site they used. Maria, a Peruvian 15-year-old from Massachusetts, refused to get on MySpace for fear that she would be kidnapped; she chose to join Facebook because she thought it was safe. While some of her classmates are on Facebook, most of the people in her community are on MySpace and they think that Facebook is "stupid." By refusing to participate, Maria was implicitly considered stupid as well. Her peers pressured her to join MySpace. Some pointed out holes in her safety logic by noting that Facebook makes users provide first and last names while MySpace does not. 
When I interviewed her, she doubted her own decision. She did not like that she was being socially ostracized for choosing the "wrong" site.

\subsubsection{Distinctions and Social Categories}

As teens began choosing between Facebook and MySpace, distinctions started to emerge between the adopter populations of each. While geography and town size did play a role in adoption patterns, teens in the same schools-and even households-began to move toward different sites. Even teens who used both sites used them to interact with different groups of people. Many of the teens I interviewed noted that the split correlated with social divisions that played out in school, with some types of teens choosing one and a different type of teen choosing the other. In trying to locate these distinctions, teens turned to terms that mark social categories. Consider the following two descriptions of MySpace and Facebook differences, as described by teens who preferred Facebook.

In an email, 17-year-old Anastasia from New York explained:

"My school is divided into the 'honors kids,' (I think that is self-explanatory), the 'good not-so-honors kids,' 'wangstas,' (they pretend to be tough and black but when you live in a suburb in Westchester you can't claim much hood), 'the latinos/hispanics,' (they tend to band together even though they could fit into any other groups) and the 'emo kids' (whose lives are alllllways filled with woe). We were all in MySpace with our own little social networks but when Facebook 
opened its doors to high schoolers, guess who moved and guess who stayed behind ... The first two groups were the first to go and then the 'wangstas' split with half of them on Facebook and the rest on MySpace ... I shifted with the rest of my school to Facebook and it became the place where the 'honors kids' got together and discussed how they were procrastinating over their next AP English essay."

Across the country in California, 17-year-old Craig Pelletier tried to locate the distinctions on his blog:

"The higher castes of high school moved to Facebook. It was more cultured, and less cheesy. The lower class usually were content to stick to MySpace. Any high school student who has a Facebook will tell you that MySpace users are more likely to be barely educated and obnoxious. Like Peet's is more cultured than Starbucks, and Jazz is more cultured than bubblegum pop, and like Macs are more cultured than PC's, Facebook is of a cooler caliber than MySpace."

Both Anastasia and Craig distinguished MySpace and Facebook participation through references to social categories, class dynamics, and stereotypes. They were not alone. White 15-year-old Kat from Massachusetts calls MySpace "the ghetto." She is reluctant to use this term in front of me because of its racist implications, which she acknowledges, but she clarifies that she does not mean it in a racist manner, but rather that Facebook is aligned with being "more mature." Melanie, the 15-year-old in Kansas, reinforces this view by pointing out that changing layouts on MySpace is "like you're a little kid on Xanga, and Facebook is more like adultness." 
The view that Facebook - and its users-are more mature is woven through many of the dialogues I had with teens who preferred Facebook.

Of course, the same qualities that signal maturity to some are seen as symbols of brownnosing to others. Penelope, the 15-year-old from Nebraska, rolls her eyes as she tells me that Facebook is for the "goody, goody kids" while everyone else is on MySpace. Other teens tell me that Facebook is "sterile" and "boring" and that those who participate lack the ability to creatively express themselves. Seventeen-year-old Martin mocked Facebook as being the trendy place for the "popular kids." He and his friends preferred MySpace, especially since it meant not having to deal with that group from school.

While the usage breakdown and dominance differed across the country, teens in vastly different communities used common language to distinguish between MySpace and Facebook and their users. In trying to map out the distinctions between the adoption of Facebook or MySpace, teens employed the language of race, class, and social categories. They recognized that their social worlds were structured by these dynamics and saw this reflected in the site distinctions. While the language teens used focused on stereotypes, the divisions they were indicating were correlated with emergent but real demographic differences in usage.

Using data collected at a diverse university in Chicago, Eszter Hargittai (2007) found that freshman site choice was correlated with race and parental education. Students from families with higher education and those who were white or Asian 
were more likely to use Facebook, while Hispanic students and those from families with less education tended toward MySpace. While Hargittai's findings are not specifically about socioeconomic class, race and education are strongly correlated with class in the United States. In San Francisco, Chuck Lam (2007a; 2007b) found that MySpace and Facebook distinctions could be found at schoolwide levels, correlated with the ranking of the school. Because school rankings often fall out along socioeconomic class levels, Lam used a school-rating service to choose a sample of public and private schools in San Francisco with different ranks and then assessed how many teens from each of those schools had MySpace and Facebook profiles. He found that students from top-rated schools were more likely to use Facebook while students from lower-ranked schools were more likely to be on MySpace. These two quantitative studies complemented what I was seeing and hearing qualitatively.

Adult society also reinforced divisions through the attitudes it maintained toward these sites. Throughout my fieldwork, MySpace was criticized by mainstream media and among parents while Facebook was valorized, even when both sites enabled the same practices that worried adult society. What effect this had on youth adoption is unknown, but adult biases were prevalent throughout my fieldwork.

\subsubsection{Reinforcing Offline Social Categories}

The split between MySpace and Facebook is visible along the lines of race, class, and education levels, but fundamentally, the split is driven by social categories. As 
Penny Eckert (1989) notes, race, class, and education levels configure social categories. Using Eckert's language, Facebook became the site for the "jocks" while MySpace attracted the "burnouts." Just as the vast majority of teens are not truly jocks or burnouts, the vast majority of teens are not cleanly defined by either MySpace or Facebook. They move between both, trying to find their place. Yet each site still represents the core archetypical social categories described by Eckert, and those who opt for one tend to mock those who adopt the other.

The online divisions mirror broader offline divisions surrounding social categories that are persistent in schools today. Some of this can be seen through teens' usage of labels. While the practice is not universal, teens often used labels to demarcate outcasts (e.g., "gothics," "nerds," "Dirty Kids," etc.). Teens also grouped people by shared activity and labeled them with terms that referred to the activity (e.g., "band kids," "art kids," "cheerleaders," etc.). When teens did use labels, they often felt the need to describe them to me. For example, Heather, a white 16-yearold from Iowa, explained: "You've got the pretties, which are the girls that tan all the time. They put on excessive makeup. They wear the short skirts, the revealing shirts, that kind of things. Then you've got the guys who are kind of like that, dumb as rocks by the way." By and large, teens did not identify themselves through labels except to indicate how others referenced them. When teens did take on labels, they were predominantly driven by activity. For example, Clyde, a Hispanic-white 16-year-old from Michigan, located his own identity when listing off the cliques at his school, relying heavily on activity markers: 
“There's the band geeks, which I'm part of. There's the weird kids, which they've deemed themselves weird. There's kids that dress up like ghetto. There's kids that dress up like gothics, but they're not. They're posers. You can even tell. Then there's choir geeks. Then there's theater geeks. Then there's art geeks. Then there's the jocks and then there's the cheerleaders."

Race, gender, and class are also frequently employed when teens divide their peers. For example, I heard labels like "boy cheerleaders" and "girl jocks." These terms add gender to an activity-centered approach in a manner that indicates underlying assumptions about normative activity membership. While gender is usually added to other markers, race tends to be referenced as a category of its own. Throughout my fieldwork, I heard labels like "the Chinese people," "the Hispanics," "the blacks," "the Mexicans," "the white people," and so forth to describe specific social groups. In schools where a particular racial group clearly dominated, that group went unmarked, but otherwise, race was tremen dously visible.

When I asked teens about why friend groups are defined by race, they typically shrugged and told me that that is just the way it is. Traviesa, a Hispanic 15-year-old from Los Angeles, articulated this best when she told me, "If it comes down to it, we have to supposedly stick with our own races.... That's just the unwritten code of high school nowadays." Race was not an issue only in major metropolitan communities. Heather, the 16-year-old in Iowa, told me that her school was not segregated, but then she went on to mark people by race, noting that "the black kids are such 
troublemakers." It became clear that while there was no formal segregation, the black teens in her dominantly white school stuck together socially and were marked as a social group. Because social categories are frequently structured by race, the divisions around social categories reinforce racial stereotypes.

Unlike race and gender, class markers tended to be much more implicit except for labels like "the rich kids." For example, teens in Kansas told me about the "dirty kids" who never seemed to shower, change their clothes, or get haircuts. When I met with teens whom others labeled as "DKs," it became clear that the factors that resulted in their being perceived as "dirty" were primarily driven by poverty.

While race, class, and gender have long been seen as salient to the construction of friend groups (McPherson et al. 2001), social categories (Eckert 1989), and tastes (Bourdieu 1984), I also found that religion is sometimes employed in ways that are similar to how race is used to demarcate groups and segregate populations. In particular, I was surprised by how frequently non-Muslim teens talked about "the Muslims" when talking about groups at school. In two communities, teens unabashedly told me that Muslim teens were usually labeled "the terrorists." While I did not analyze the bigotry that I witnessed, the presence of these labels surprised me.

While shared activities and identity markers are one way that teens can demarcate friend groups and social categories, it is more common for them to do so by social network or geography. For example, Sara, the 16-year-old from Austin, told 
me that she could describe friend groups at her school by listing people by name. Yet more frequently teens told me that the way to reference friend groups was by talking about lunch tables at school. For example, Myra, the 15-year-old from Iowa, and white 14-year-old Leigh, also from Iowa, told me that they could not tell me the names of different cliques in their school, but that they could see them in the lunchroom. Teens gather with friends in the morning and sit with friends at lunch. Most of the teens I interviewed sit at the same lunch table every day and, as Hollie, a white 15-year-old from Pennsylvania, points out, "There are different tables that have different groups of tables." By and large, these tables are segregated by social categories and grade. The most notable exception occurs when differently labeled outcasts all sit together.

In some schools, the organization of turf goes beyond the lunchroom. When I interviewed Keke, the 16-year-old from Los Angeles, she mapped out where different groups hang out during breaks:

"This school is so segregated, it's crazy. We got 'Disneyland' full of all the white people. ... The hallways is full of the Indians, and the people of Middle Eastern decent. They in the hallways and by the classrooms. The Latinos, they all lined up on this side. The blacks is by the cafeteria and the quad... Then the outcasts, like the uncool Latinos or uncool Indians, the uncool whites, they scattered."

Divisive social categories_-often driven by race, gender, and class—are a salient part of teens' lives. These are replicated and reinforced online. Just as teens divide 
courtyards and lunchrooms based on social categories, so too do they divide digital environments. The division between MySpace and Facebook is more than a reproduction of preference; it is a reproduction of turf wars that play out as a result of social categories. Furthermore, as shown by the media coverage of these two sites, it is a reproduction of cultural fears and anxieties around race, class, gender, and the social categories that are driven by these factors.

\subsubsection{Status and Digital Fashion}

As adoption patterns unfolded, MySpace and Facebook became a form of digital turf framed by social categories. While there are functional differences between the sites, they are relatively limited. When I asked teens about functional differences, their comments quickly moved to aesthetics and profile personalization. In essence, the "glitter" produced by those who "pimp out" their MySpaces is seen by some in a positive light while others see it as "gaudy." While Facebook fans loved its aesthetic minimalism, others viewed this tone as boring, lame, and elitist. The aesthetics issues that teens mark further reflect the differences in the core social categories represented by MySpace and Facebook.

Catalina, the 15-year-old from Austin, told me that Facebook is better because "Facebook just seems more clean to me." What Catalina sees as cleanliness, Anindita, the 17-year-old from Los Angeles, frames as simplicity; she recognizes the value of simplicity, but she prefers complexity. "Facebook's easier than MySpace but MySpace is more complex. You can add things to it. You can add music, make 
backgrounds and layouts, but Facebook is just plain white and that's it." While Anindita loves being able to express herself through MySpace, the extensive options for self-expression are precisely what annoy other teens. Craig Pelletier, the 17-yearold from California, complained that

"these tools gave MySpacers the freedom to annoy as much as they pleased.

Facebook was nice because it stymied such annoyance, by limiting individuality.

Everyone's page looked pretty much the same, but you could still look at pictures

of each other. The MySpace crowd felt caged and held back because they weren't able to make their page unique."

For Craig, the desire to personalize is what divides his peers between MySpace and Facebook.

Profile personalization is a form of digital fashion. Fashion and aesthetics are rooted in identity, class, and social grouping. Style is an important marker of identity (Davis 1992; Hebdige 1979), and people are drawn to styles that signal their identities and social groups. The social agendas around race and class also drive fashion (Crane 2000). The distinctions people make about taste reflect their relationship to socioeconomic status and status is reinforced through distinctions in taste (Bourdieu 1984). These same issues play out online. The valued minimalism of Facebook is not inherently better, although conscientious restraint has been one marker of bourgeois fashion (Arnold 2001). On the contrary, the flashy style that is popular on MySpace has a lot in common with "bling-bling," a style of conspicuous 
consumption that is associated with urban black culture. To some, bling and flashy MySpace profiles are beautiful and creative; to others, these styles are gaudy. The division is not a matter of universal aesthetics but of the cultural signals implied by identifying with different aesthetic approaches.

Fashion is one of the most visible ways in which teens seek to stand out and fit in (Milner 2004). It is how teens mark social categories, subcultures, and peer affinity. Not surprisingly, these same practices that play out in social groupings in unmediated spaces are also playing out online. The choice of MySpace vs. Facebook fractured teen culture. As the popularity of these sites unfolded, MySpace and Facebook became synonymous with different social categories. As teens chose stylistic approaches that reflected their peer structures and located themselves in relation to these sites, they identified with and reinforced the cultural divisions and social categories that these sites represented. Their affinity for the different aesthetics presented by these sites reveals the ways in which fashion continues to be a marker of social categories, even in the digital era. In essence, MySpace and Facebook became a new type of turf-digital turf on which teens' struggles for status and identity play out.

\subsection{Public Displays of Connection and Status}

Small children often seek confirmation of friendships through questions such as "We're friends, right?" (Corsaro 1997: 164). Yet in everyday life most teen friendships are never formalized or verified except through implicit social rituals. 
Social network sites change this by forcing participants to publicly articulate and display their social connections. Not only does this feature affect how teens negotiate friendships, but it also complicates teens' relationship to peer status.

While these lists display "Friends," the connections teens have on a social network site are not necessarily the same as "friends" in the everyday sense (boyd 2006b). The reasons to connect with others are countless and participants use the term "Friends" to label all connections, regardless of intensity or type (e.g., family, colleagues, etc.). Part of what makes this feature complicated is that choosing whom to select as Friends can be socially challenging. Teens must struggle to decide whom they wish to include and exclude. Their decision is more than personal, though—all of those they choose to include are publicly displayed in connection with their profile and those that they exclude are not. MySpace and Facebook do not allow directed connections. When someone decides to add someone else as a Friend, a Friend request is sent. That request must be confirmed or denied or else it lingers in limbo. Only connections that are confirmed appear as Friends; ignored and rejected connections do not.

The Friends list feature serves multiple purposes. At a social level, this feature is a formal structure for marking oneself in relation to others within the system and working out the status dynamics involved in peer relations. Functionally, the Friends list plays multiple roles. As an address book, this list provides an easy way to maintain a record of relations. As an access-control list, it allows teens to leverage 
privacy settings to control who can access their content, who can contact them, and who can see if they are online or not. It also provides a mechanism by which teens can navigate the social structure. Finally, the public display of connections that takes place in social network sites can represent a teen's social identity and status(Donath and boyd 2004). As such, it places an individual within a social context, while also serving to help define that context in important ways.

\subsubsection{Strategies for Friending}

Teens have different strategies for choosing whom to mark as Friends. By and large, the teens I interviewed "Friend" those they know—friends, family, peers, and so on. Yet, even within the confines of this general rubric, there is immense variation. Teens may choose to accept requests from peers they know but do not feel close to if only to avoid offending them. They may also choose to exclude people they know well but do not wish to connect with on Facebook or MySpace. This category rarely includes peers, but it often involves parents, siblings, and teachers. Both MySpace and Facebook offer many incentives for adding people other than close friends. Many of the privacy features that were introduced during the course of my study limit non-Friends from profile viewing, leaving comments, and, in some cases, sending messages. Teens who wish to talk with peers or friends of friends are encouraged to accept requests from peers so as to open the channel of communication. 
Teens must determine their own boundaries concerning whom to accept and whom to reject. For some, this is not easy. Generally, there are common categories of potential Friends that most teens address in deciding how to structure their boundaries. The first concerns strangers. While many early adopters of MySpace gregariously welcomed anyone and everyone as Friends, the social norms quickly changed. For most teens, rejecting such requests is now the most common practice. Although teens who accept Friend requests from strangers rarely interact with these people online, let alone offline, the same concerns that keep teens from interacting with strangers online also keep them from including strangers in their lists of Friends. Like many teens, Guatemalan-Pakistani 15-year-old Ana-Garcia from Los Angeles adds only people she knows as Friends. She does not want strangers on her Friends list and she is adamant that her sister does not have strangers either. Her approach reifies MySpace's claim that it is "a place for friends."

While most teens focus on Friending people they know, some teens actively connect with strangers. Teens commonly send Friend requests to bands and celebrities. They do not believe that such connections indicate an actual or potential friendship, but they still find value in these Friends. Other teens seek strangers who share their interests, primarily around music. For example, Eduardo, a Hispanic 17year-old from Los Angeles, leverages MySpace Music to make his rap music available to a wider audience. He loves that he can share his music with his friends, but he especially likes that there is the potential to meet other musicians or people who might help him produce his music. Another musician, Dom, the 16-year-old from 
Washington, has actively used MySpace to connect to other musicians. Through MySpace, he found another musician who shared his musical interests and they recorded music together. Teens who have passionate interests can and do use social network sites to meet others who share their interests.

Some participants go beyond the occasional connection and collect strangers as Friends. The vast majority of those who do this are adults-musicians, politicians, corporations, and wannabe celebrities. Teen musicians and activists sometimes collect Friends-a.k.a. fans-for the same purposes as adults. Teens also collect as a form of entertainment or competition among friends. Some collect fake profiles. For example, Heather, the 16-year-old from Iowa, is Friends with various characters from TV shows and a Fiji Water bottle-she knows that they are not real, but she thinks that such fake characters are entertaining. Collecting fake profiles has a long history on social network sites (boyd 2008b) and it is often a form of entertainment. The teens who collect strangers or fake entities are typically not interested in developing friendships with these people; they simply collect them because it is something to do. Of course, some teens do collect in the hopes that a Friend connection will evolve into something more. One boy said that it is fun to see which attractive women would say yes to his Friend requests. Spammers have started making profiles of attractive women to lure males, knowing that they are often receptive to such potential connections. 
One reason that teens collect Friends is as a marker of status. Ty, the 17-year-old from Los Angeles, explained that he likes to have a lot of Friends on his MySpace "to show that, you know, you're popular or something like that." Connecting with strangers, especially attractive women, allows him to create a profile that appears cool. Yet this is precisely why other teens criticize the practice of collecting Friends. Mark, a white 15-year-old from Seattle, complains that "there's all these people that judge [MySpace] as a popularity contest and just go around adding anyone that they barely even know just so they can have like, you know, 500,000 friends just because it's cool. I think that's stupid, personally." Such collectors are sometimes derogatively called "MySpace whores." While this term is both gendered and sexualized in nature and those loaded references are sometimes intended, it is applied to both boys and girls and refers to attention seekers of all types, not just those seeking sexual attention. Some teens find the attention-seeking nature of Friending problematic. Aggravated by her peers' use of MySpace as a popularity contest, Samantha, the 18year-old from Seattle, decided to alter her MySpace profile so that it would say that she had thousands of Friends. She did this "to mock other people."

While connecting with strangers is controversial, there is little social cost to rejecting Friend requests from strangers-because these people are unknown, teens do not worry about offending them. Rejecting known individuals, on the other hand, is much more complicated. So while teens differ on whether or not to connect with strangers, they generally accept Friend requests from all known peers, including all friends, acquaintances, and classmates, regardless of the quality of the 
relationship. Jennifer, a white 17-year-old from a small town in Kansas, upholds this social convention because "I'd feel mean if I didn't." She sees Friend requests as a sign of niceness and the opening of potential friendships. She also thinks it is important to be nice because she would be "mad" if someone rejected her attempt to be nice.

Incentives for Friending all peers extend beyond the social cost of rejection. In Iowa, white 17-year-old Sam told me that the reason teens add people as Friends is the same reason that they try to make lots of friends- "because they want to seem popular." Having lots of connections is socially valuable, both for reasons of status and to be in the loop. Sam thinks that teens try to have lots of Friends because:

"They want to have their foot in everything that's going on and people like to be informed and they like to know the gossip or what's happening with so-and-so and you can't really go up to someone and ask them if you don't know them. So you kind of have acquaintances all over the place that help keep you in the know."

Social network sites take this to the next level because Friending can be both a symbolic display of popularity and a functional way of gaining access to what is being said.

While Friending classmates can lay the groundwork for building a friendship out of an acquaintanceship, not all such Friend requests are attempts to deepen the relationship. Often teens send requests to everyone they know or recognize and no additional contact is initiated after the Friend request is approved. This only adds to 
the awkwardness of the Friend request. As Lilly, the 16-year-old from Kansas, explains, getting Friend requests from classmates does not mean that they even know who she is at school, making it difficult to bridge the online-offline gap:

"It's just on Facebook, you're friends. At school, you don't have to talk if you don't want to.... It's kind of nice, but then at the same time it's not because you know they're your Friends. ... You don't say hi in the hall 'cause maybe they just added me because somebody else had me added and they'd be like, 'I don't know who you are. Hi."'

Lilly accepts requests from all classmates, even those who she is not sure know who she is, but her friend, the 15-year-old Melanie, prefers to mock the dynamic that this sets up. Melanie will approach classmates who send her Friend requests with comments such as "Hey Friend from Facebook" simply because she thinks it is funny. Melanie's approach to Facebook is quite unusual. Not only is she willing to call out the absurdity of being Friends online but not talking at school, but she is also willing to buck the norms by rejecting people she does not like and deleting people who annoy her. Melanie notes that Facebook "is better than real life" because while there is no simple mechanism to formally indicate disinterest in school, it is possible to say "No" on Facebook by rejecting Friend requests. Likewise, when people annoy her on Facebook, she is comfortable deleting them. While Melanie and Lilly both find the online Friending practices to be "fake," Melanie is more outraged. Both girls are in the top classes at school and involved in many activities, but neither is 
particularly popular. I get the sense that Melanie's resentment stems from her frustration with the status games and peer pressure that take place at school. Melanie is adamant to point out that she does not drink or party; she thinks teens should be more focused on what is "important."

Most teens find deleting people discomforting and inappropriate. Penelope, the 15-year-old from Nebraska, says that deleting a Friend is "rude ... unless they're weird." Yet while she will do it occasionally, the process of deleting someone is "scary" to Penelope; she fears that she will offend someone. Generally, it is socially unacceptable to delete a Friend whom one knows. When this is done, it is primarily after a fight or breakup. In these situations, the act of deletion is spiteful and intentionally designed to hurt the other person. Teen awareness of malicious deletions adds to the general sense that deleting someone is socially inappropriate. Thus, it can be problematic when teens accidentally delete people they know. AnaGarcia, the 15-year-old from Los Angeles, faced this problem when her brother decided to $\log$ in to her account and delete two pages' worth of Friends. Luckily, those she did know understood as soon as she explained what happened. While deleting known people can be seen as malicious, it is socially acceptable to delete strangers. In fact, there is often social pressure to do so. Lolo, the 15-year-old from Los Angeles, says: "At the beginning, I was just adding people just to get Friends and just random boys living in New York or Texas. Then my boyfriend kinda like, 'You don't know them ...' so I deleted them and then I had 300 and I really knew them." 
By forcing them to articulate relationships, the Friends feature forces teens to navigate their social lives in new ways. While teens are developing a set of shared social practices for Friending, the norms for these practices are still in a state of flux and interpretive flexibility. The process of adding and deleting Friends is a core element of participation on social network sites. It allows teens to negotiate who can gain access to their content, but it also means that teens have to manage the social implications of their decisions. Because the peer groups that teens connect with on social network sites are the same as those they socialize with in everyday life, decisions about whom to accept and reject online directly affect their offline connections. By facing decisions about how to circumscribe their Friends lists, teens are forced to consider their relationships, the topology of their peer group, and the ways in which their decisions may affect others.

\subsubsection{Hierarchies of Friends}

Friending forces teens to be explicit about their connections to others. Yet an additional feature on MySpace_- "Top Friends" (formerly “Top 8”)—-takes this to another level by requiring teens to rank their Friends. While teens can avoid offending classmates by accepting all Friend requests from peers, the ranking feature complicates teens' relationships with their friends. In essence, this feature requires teens to list their best and "bestest" Friends. Because there is often no socially appropriate way to rank order close friends, many teens blame this feature for the 
proliferation of social drama on MySpace. Choosing whom to display is fraught and teens struggle to find ways to manage this without doing social damage.

Rhetoric such as "best friends forever" ("BFF") is common among children, especially young girls (Thompson et al. 2001: 62). This stems from a desire by children to understand the strength of their relationships and embedded in this is an expectation of affirmation and reciprocity. Most friendship declarations take place verbally between friends, but girls have used symbolic accessories such as "BFF" heart charms and friendship bracelets to formalize and display their connection. While these practices exist, they are far more common with elementary-school children and middle-school tweens than with teenagers. The idea of "best friends" does not disappear in high school, but the formal symbolism fades. In many ways, MySpace's Top Friends feature reintroduces the formal symbolism to an older crowd.

Top Friends was designed to help participants add nuance to their Friends list, but it quickly became a social battleground as participants struggled over who should make the list and, more important, who should be in the first position. As Anindita, the 17-year-old from Los Angeles, explains:

"People will be like, 'Why am I number two? You're number one on my page.' I was like, 'Well, I can't make everyone number two. That's impossible.' Especially with boyfriends and girlfriends, get in a fight like, 'Why is she before me? I'm your girlfriend. I should be higher than her.' I'm just like, 'Okay.' I don't really think it's 
a big deal, the top thing. If you're friends, you shouldn't lose your friendship over that."

Like many teens, Anindita finds the social dynamic around Top Friends frustrating, but she is not immune to its effects. Even though she thinks it should not be important and although she could avoid it on her own page, this feature is a topic of regular conversation among her friends. While Anindita may see her friends' attitude as cattiness, Top Friends plays into insecurities by forcing teens to face where they stand in the eyes of those around them. Top Friends interferes with the way in which teens normally manage relations that they would not normally declare or rank. As Nora, a white 18-year-old from Virginia, explains on her MySpace:

"It's like have you noticed that you may have someone in your Top 8 but you're not in theirs and you kinda think to yourself that you're not as important to that person as they are to you ... and oh, to be in the coveted number-one spot!"

Many teens see the Top Friends feature as a litmus test of their relations and this prompts anxieties about where they stand.

Reciprocity plays a central role in the negotiation of Top Friends. Many teens expect that if they list someone as a Top Friend, that person should do so in return. Teens worry about not being listed and about failing to list those who list them. Jordan, the 15-year-old from Austin, says, "Oh, it's so stressful because if you're in someone else's [Top Friends] then you feel bad if they're not in yours." The struggles 
that teens face in constructing their Top Friends resemble those involved in choosing whom to invite for a special occasion. As Nadine, a white 16-year-old from New Jersey, describes on her MySpace:

"As a kid, you used your birthday party guest list as leverage on the playground. 'If you let me play I'll invite you to my birthday party.' Then, as you grew up and got your own phone, it was all about someone being on your speed dial. Well, today it's the MySpace Top 8. It's the new dangling carrot for gaining superficial acceptance. Taking someone off your Top 8 is your new passive-aggressive power play when someone pisses you off."

While there are parallels between Top Friends, speed dial, and the birthday party, there are also differences. Top Friends are persistent, publicly displayed, and easily alterable. This makes it difficult for teens to avoid the issue or make excuses such as "I forgot." When pressured to include someone, teens often oblige or attempt to ward off this interaction by listing those who list them. Catalina, the 15-year-old from Austin, says: "If you're in someone else's, you have to put them in yours." Other teens avoid this struggle by listing only bands or family members. While teens may get jealous if other peers are listed, family members are exempt from the comparative urge. This is the strategy that Traviesa, the 15-year-old from Los Angeles, takes to avoid social dramas with her friends:

"It's very difficult to choose a Top 8 because when you do your friends are like, 'Well, why didn't you choose me?' And this and that, and I'm like, 'Well, all right 
fine, I'll just choose, 'like I choose my cousins now because I can't deal with it. Like everybody's always like, 'Why didn't you put me on, why am I not on your Top 8? You're on mine."'

In addition to having to decide whom to include, teens must also decide in which order those Friends will be listed. The most valuable position—the "first" one-is the one in the upper left corner of the grid. This position is usually reserved for a person's "best" friend, significant other, or a close family member. While few object to a significant other's appearing first, some teens, especially girls, get jealous when other same-sex peers are listed above them on the page of the person they believe to be their closest friend. Exceptions are made for family members and it is common in some teen circles to list family first. While some teens list family to avoid conflict with friends, others do so because they see a family member as their closest friend. This is exemplified by Laura, a white 17-year-old with Native American roots from Washington, who says: "My sister is in position number one because she is one of my best friends and she will be there for me most likely longer than anyone else."

Because of the ways in which Top Friends collapses the complexities of social relations and hierarchies, teens have developed a variety of strategies that govern what is and is not appropriate. While common practices ease some tensions, the Top Friends feature still causes anxieties and social pressures. Most of these stabilize through time but not without a few battle scars. 
Although most teens find a way to manage the Top Friends feature, others prefer to avoid it altogether. Some intentionally leave the site's founder in the first position while others find more creative solutions. One teen explained that she changed her Top Friends every month, creating themes such as "all Sagittarius Friends." After getting frustrated with the resultant social dramas, Amy, a black-white 16-year-old from Seattle, found code that allowed her to not display her Top Friends on her profile so that no one could see them and, thus, no one could be upset with her. While Amy's approach is uncommon, it highlights the power of this feature in shaping how teens interact with the site.

Not all teens participate in the social dramas that result from Top Friends, but it does cause tremendous consternation for many. The Top Friends feature is a good example of how structural aspects of software can force articulations that do not map well with how offline social behavior works. Top Friends suggests a single, context-free, hierarchical ranking of friends and a hard cut between "Top" friends and everyone else. This results in social drama for multiple reasons. First, teens do not necessarily think of their friends as hierarchically ranked, but the technology forces this ranking. Second, teens might feel closer to different friends in different contexts and along different dimensions. Friends from a sports team might be different from friends in geometry class. All of those situational distinctions are erased in the Top Friends feature. As a result, friends from different contexts are forced into a single spot for comparison. Finally, teens might feel close to some friends because they get them invited to parties and close to other friends because 
they help them with their homework. Collapsing these different dimensions only adds to the difficulties teens have when struggling with status among peers at school (Milner 2004).

The process of articulating and ranking Friends is one of the ways in which social network sites take what is normally implicit and make it explicit. When teens are enmeshed in dramas about social categories, cliques, and popularity, the forced nature of Friending can be turbulent. Like the practices of accepting or rejecting Friend requests, the practices of ranking Friends translates certain forms of social connectedness into an online representation. The problem with explicit ranking, however, is that it creates or accentuates hierarchies where they did not exist offline, or were deliberately and strategically ambiguous, thus forcing a new set of socialstatus negotiations. The give-and-take over these forms of social ranking is an example of how social norms are being negotiated in tandem with the adoption of new technologies, and how peers give ongoing feedback to one another as part of these struggles to develop new cultural standards.

\subsection{Status Battles and Peer Dramas}

As core social hangout spaces for teens, social network sites are home to the struggles that teens face as they seek status among peers (Milner 2004). Teens use social network sites to develop and maintain friendships, but they also use them to seek attention and generate drama among peers. Often the motivation behind the latter is to relieve insecurities about popularity and friendship. While teen dramas are 
only one component of participation on social network sites, they are often the most visible and memorable component. The persistent and networked aspects of social network sites alter and amplify the ways that these dramas play out in teen life. For this reason, it is important to pay special attention to the role that social network sites play in the negotiation of teen status.

Teens seeking to spread rumors or engage in drama use every channel of communication available. These acts may be lightweight parts of everyday teen life or they may snowball in magnitude and become acts of bullying. Regardless of the intensity, what is most noticeable is that the acts of drama involving social network sites are primarily a continuation of broader dramas. Stan, a white 18-year-old from Iowa, says, "You'd actually be surprised how little things change. I'm guessing a lot of the drama is still the same; it's just the format is a little different. It's just changing the font and changing the background color really." While the underlying practices may be the same, Michael, the 17-year-old from Seattle, points out that MySpace amplifies dramas because it extends social worlds beyond the school:

"MySpace is a huge drama maker, but when you stick a lot of people in one thing, then it's ... it always causes drama. 'Cause, like ... MySpace is, like, a really big school. ... School's filled with drama. MySpace is filled with drama. It's just when you get people together like that, that's just how life works, and stuff."

The unique features of networked publics-persistence, searchability, replicability, invisible audiences, and scalability—can alter the visibility of these 
dramatic acts, making it much more difficult for participants to gain a complete picture of what is happening or to interpret the acts accurately. Gossip and rumors have played a key role in teen struggles for status and attention since well before the Internet (Milner 2004). Furthermore, other channels of social media are well documented as being conduits of gossip and drama (Baron 2008; Grinter and Eldridge 2001; Grinter et al. 2006). When teens gather with friends and peers, they share stories about other friends and peers. While it is unclear whether or not the Internet has changed the frequency of gossip, social media certainly alters the efficiency and potential scale of interactions. Because of this, there is greater potential for gossip to spread much further much faster. While teen gossip predates the Internet, some teens blame the technologies for their roles in making gossip easier and more viral. The properties of the Internet that allow information to flow more efficiently also allow it to be a culprit in teen drama.

Social network sites provide another stage on which dramas can be played out. Some of these dramas are truly dramatic, while others are mundane parts of everyday life. When content is persistent (e.g., comments on social network sites), teens can gain access to the gossip even when they were not present for the situation being referenced. The public nature of social network sites, in particular, makes it much easier for teens to "overhear" what is being said. Furthermore, because teens' presence as observers may not be noticeable online, social network sites can allow them to "stalk" their peers, keeping up with the gossip and lives of people they do not know well but with whom they are familiar. Penelope, the 15-year-old from 
Nebraska, says, "If [the popular kids are] having a fight you know about it. They confront each other. They say, 'Well, if you're going to leave a comment like that on her page then you'd better send a comment to everybody because this is a war,' or something like that."

While teens can surf through their Friends' profiles to read their comments, Facebook introduced a feature in September 2006 that made this process much easier: the "News Feed." When teens log in to their Facebook, they are presented a News Feed that lists actions taken by their Friends on the site. Some of the actions that are announced on the News Feed include: when two people become Friends, when someone leaves a comment on someone else's wall, when a Friend uploads new photos, and when two people break up. Although teens can opt out of this, many of them do not, either because they do not know about the option or because the juicy updates are too alluring. The News Feed draws on teens' desire to consume gossip about those they know (boyd 2008a).

Cachi, a Puerto Rican 18-year-old from Iowa, finds the News Feed useful "because it helps you to see who's keeping track of who and who's talking to who." She enjoys knowing when two people break up so that she knows why someone is upset or when she should reach out to offer support. Knowing this information also prevents awkward conversations that might reference the new ex. While she loves the ability to keep up with the lives of her peers, she also realizes that this means that "everybody knows your business." While Cachi values the News Feed, some teens 
find it annoying or irrelevant. Gadil, an Indian 16-year-old from Los Angeles, thinks that it is impersonal while others think it is downright creepy. For Tara, the Vietnamese 16-year-old from Michigan, the News Feed takes what was public and makes it more public. "Facebook's already public. I think it makes it way too like stalker-ish." Her 18-year-old sister, Lila, concurs and points out that it gets "rumors going faster." Kat, the 15-year-old from Massachusetts, uses Facebook's privacy settings to hide stories from the News Feed for the sake of appearances.

As a feature that amplifies public acts, Facebook's News Feed helps rumors posted publicly to spread further faster. Yet according to the teens I interviewed, the vast majority of rumors spread through more private channels such as IM and text messaging. IM allows teens to converse with multiple people at once and to copy and paste conversations to spread information. Through forwarding, text messaging can help create gossip chains. Thus, even though these channels may be more "private," information can become public through incessant sharing.

While gossip is fairly universal among teens, the rumors that are spread can be quite hurtful. Some of this escalates to the level of bullying. Measuring "cyberbullying" or Internet harassment is difficult, in part because both scholars and teens struggle to define it. The teens I interviewed spoke regularly of "drama" or "gossip" or "rumors," but few used the language of "bullying" or "harassment" unless I introduced these terms. When Sasha, a white 16-year-old from Michigan, was asked specifically about whether or not rumors were bullying, she said: 
"I don't know, people at school, they don't realize when they are bullying a lot of the time nowadays because it's not so much physical anymore. It's more like you think you're joking around with someone in school but it's really hurting them. Like you think it's a funny inside joke between you two, but it's really hurtful to them, and you can't realize it anymore."

Sasha, like many of the teens we interviewed, saw rumors as hurtful, but she was not sure if they were bullying. Some teens saw bullying as being about physical harm; others saw it as premeditated, intentionally malicious, and sustained in nature. While all acknowledged that it could take place online, the teens I interviewed thought that most bullying took place offline, even if they talked about how drama was happening online.

When teens told me about being bullied, they did not focus on the technology. They were distressed that others—often, former friends—-were maliciously spreading rumors about them to others at school. For example, Summer, the 15-year-old from Michigan, described how her best friend decided to reject her because she was not popular enough. Her former friend began by spreading secrets, but these quickly got modified and exaggerated as they spread. Summer did not know how the rumors were spreading, but she knew that everyone in school knew them fast and that many believed them. In Summer's eyes, the bullying that she experienced took place offline. Yet she also acknowledged that IM was extremely popular among her classmates at the time. It is likely that some of the rumors may have spread through 
IM or phone conversations in addition to conversations in school. For Summer, it did not matter whether it was online or offline; the result was the same. In handling this, she did not get offline, but she did switch schools and friend groups.

Media convergence complicates bullying dynamics. Both offline and online elements played a role in many of the stories that I heard. When teens are harassed online, it is often by people they know offline. Cruelty that takes place offline is often fueled by mediated rumors. Technology provides more channels through which teens can potentially bully one another, although it is not clear whether or not bullying is on the rise as a result. Studies on Internet bullying continue to show that bullying is primarily linked to various psychosocial issues, including substance use and school problems, regardless of whether the bullying takes place online or off (Hinduja and Patchin 2008; Wolak et al. 2007). Most of the teens I interviewed conceded that technology could amplify bullying, but they did not believe that technology is the root cause of it.

While bullying exists, the teens I interviewed did not see it as commonplace. They did, though, see rumors, drama, and gossip as pervasive. The distinction may have more to do with language and conception than practice. Bianca, a white 16year-old from Michigan, sees drama as being fueled by her peers' desire to get attention and have something to talk about. She thinks the reason that people start drama is that they are bored. While drama can be hurtful, many teens see it simply as part of everyday social life. 
Teens were also quick to point out that most drama and gossip comes primarily from girls, not boys. As Penelope Eckert (1996) notes in her study of girls who are transitioning to middle school, adolescent girls take on the role of "heighteners of the social." Mark, the 15-year-old from Seattle, explains that drama happens more often with girls "because they always take it more seriously." While girls are more likely to be agents in talking about drama, boys are frequently cited as the cause. A lot of drama that takes place involves crushes, jealousy, and significant others. For example, girls get upset when their friends text message or IM their boyfriends or leave comments on their social network site profiles. In general, using technology to communicate with someone who is not single can be seen as an affront.

Anindita, the 17-year-old from Los Angeles, recounted the story of how she stopped speaking to her former best friend, Meghana. Anindita was dating a boy and Meghana started telling him privately to break up with her, even though they were supposedly friends. One day, Anindita's boyfriend showed her a text message he received from Meghana. The message read, "You're the guy I love and you don't understand." This angered Anindita and she ended the friendship. From Anindita's point of view, social media takes what she sees as typical "Indian drama" and magnifies it out of control. She thinks that her peers enjoy the opportunity to start a fight for no reason other than because it is possible.

Although some drama may start out of boredom or entertainment, it is situated in a context in which negotiating social relations and school hierarchies is part of 
everyday life. Teens are dealing daily with sociability and related tensions. Lila, the 18-year-old from Michigan, sees drama as the substance of daily life while her 16year-old sister Tara thinks that it emerges because some teens do not know how to best negotiate their feelings and the feelings of others.

danah: Do you think that drama has value?

Lila: You have something to talk about.... And you're like, you want to fit in, kind of thing. You know, like way back when, when you don't know who you are, kind of. Not like I know now, but you know, when you're in middle school.

Tara: You have something to do, like to be honest, to resolve.... You feel like you're mad at somebody and you don't know how to handle it. So you just kind of turn on them like that. So it's just like, just not like having enough experience with dealing with things.

Many teens are insecure about their friendships, unsure of whether or not friends are truly loyal and trustworthy. While social network sites can complicate interactions, especially when things are already heated, they can also be used to try to ease tensions between friends. Teens can use social network sites to publicly validate one another and reaffirm a friendship. Teens use these sites to reassure their friends that they are still thinking of them. So while drama is common, teens spend much more actual time and effort trying to preserve harmony, reassure friends, and reaffirm relationships. This spirit of reciprocity is common across social network sites. Trying to be nice when someone else is being nice is one example of how this 
plays out. Penelope, the 15-year-old from Nebraska, believes in responding to comments because "if someone's nice enough to say something to you then you have to be nice enough to say it back."

Others view the social script of reciprocity from a more cynical angle, believing that their peers are being selfish when they leave a comment. These teens believe that commenting is not as much about being nice as it is about relying on reciprocity for self-gain. While some teens leave comments to be nice, others hope that they will get comments in return. This can be viewed as selfish, but it can also be seen through the lens of insecurity. Many teens worry that they may appear lame if they have too few Friends or too few comments. Some opt out because they fear that these tools would simply highlight the ways in which they are not cool. Alternatively, some who view Friends and comments as markers of social worth think they must attain these so as not to be alienated from their peers. Kevin, the 15-year-old from Seattle, believes that getting comments is cool "because it lets everyone who goes to your page know that you're not just a guy that has MySpace; you're a guy that has friends and a MySpace."

Successful participation is not simply about having an account on a social network site but about having one with status. Still, insecure and marginalized individuals sometimes seek the markers of cool even if they themselves are not actually perceived of as cool. Teens want to be validated by their broader peer group and thus they try to make themselves look cool, online and off. Even when status is 
not necessarily accessible to them in everyday life, there is sometimes hope that they can resolve this through online presentations. While some teens are happy to attain status solely within the context of a social network site, most hope that if they look cool online, their peers will notice and validate them. This strategy often is not successful. Dominic, a white 16-year-old from Seattle, says:

"I don't really think popularity would transfer from online to offline because you've got a bunch of random people you don't know; it's not going to make a difference in real life, you know? It's not like they're going to come visit you or hang out with you. You're not a celebrity or something."

Achieving status purely through social network site participation may not be viable, but participating and being popular online can complement offline popularity. Just as having the "right" clothes or listening to the "right" music can be an indicator of status in everyday peer groups, participating in the "right" social media in a manner that is socially recognized is often key to offline status. As with clothes and music, online participation alone is not enough to achieve status, but it is still important.

Gossip, drama, bullying, and posing are unavoidable side effects of teens' everyday negotiations over friendship and peer status. What takes place in this realm resembles much of what took place even before the Internet, but certain features of social media alter the dynamics around these processes. The public, persistent, searchable, and spreadable nature of mediated information affects the way 
rumors flow and how dramas play out. The explicitness surrounding the display of friendships and online communication can heighten the social stakes and intensity of status negotiation. The scale of this varies, but those who experience mediated harassment are certainly scarred by the process. Further, the ethic of reciprocity embedded in networked publics supports the development of friendships and shared norms, but it also plays into pressures toward conformity and participation in local, school-based peer networks. While there is a dark side to what takes place, teens still relish the friendship opportunities that social media provides.

\subsection{Peer Sociality in Networked Publics}

Peer relations have been and continue to be key organizing features of teen life. The popularity of social network sites and other networked publics in contemporary teen culture is intrinsically connected with teens' desire to socialize with their peers. Not only do social network sites offer an additional channel of communication for teens, they also create a networked public space in which teens can gather and socialize.

Social network sites make peer interaction possible when face-to-face encounters are not. As such, social network sites allow teens to connect across spatial and temporal barriers. As Laura, the 17-year-old from Washington, explains, "MySpace lets me talk to friends even when I can't call or see them." Sabrina, the 14-year-old from Texas, notes that her school has multiple campuses and that teens in different grade levels and classes might never see one another. She has many friends whom 
she never sees at school, but "MySpace is a good way to talk to people that I don't talk to very often at school." Because of how they are structured, social network sites have the potential to connect new people, allowing teens to socialize with those with whom they do not share geographic proximity. While the technology makes this possible, this is not normative. That said, teens do leverage the ability to enhance connections with those they already know, including those they do not know that well. In this way, social network sites alter the social relations that are realized in teens' lives.

While social network sites can and do alter the structural conditions in which teens can interact with one another, the underlying social motivations and dynamics have not changed much. Teens still gather to flirt, gossip, gain support, and work out status and identity issues. They do so in unmediated contexts and they do so online. Yet the technology also inflects these practices in unique ways. The public articulation and ranking of connections complicates social relations and status. The ability to copy and paste conversations and access interactions asynchronously can amplify the spread of gossip and magnify the cost of bullying.

Yet for all of the potential complexities, participation has become a core part of teen culture because teens value opportunities to gather with peers broadly, especially when their interactions are not heavily configured by adults. As physical spaces for peer sociability have disappeared or been restricted and as teens have found their access structurally or socially curtailed, the value of mediated spaces in 
which teens can gather has increased. Networked publics have become the modernday equivalent of the mall or movie theater, a place where teens can hang out with friends and run into other friends and peers.

Teens realize that there is a difference between one-on-one and group interactions and they value each type differently. Emily, a white 16-year-old from Pennsylvania, points out that the social possibilities of going to the mall or movies are far greater than going to a friend's house:

"If you go [out] with your friends, there might be other people you run into that are your friends too. I would say it's more of an opportunity to see more of your friends than just going over to a friend's house. Going over to a friend's house, there might be one friend or maybe three. Whereas going to the mall, it can be seven or $12 . "$

In the same way, MySpace and Facebook allow teens to go online with friends and, in the process, run into other friends and peers.

Public spaces—-whether mediated or not-provide greater opportunities for teens to make sense of the social world around them. Furthermore, these spaces allow teens to develop new connections and to flirt. Unstructured social spaces offer more opportunities for working through status issues and building relationships. Given these factors, teens seek out opportunities to gather with their peers in unstructured ways. In their choosing where to go, the presence of peers and friends is the most important. If friends and peers gather in person, teens feel the need to be physically 
there to feel included. If the gathering takes place online, being in that space online becomes socially critical.

The key teen social practices have not changed as a result of technology, but the site of gathering has. In learning how to use social network sites and facing decisions about which site to join, how to Friend, and how to behave appropriately, teens are learning to negotiate peer relations, social status, and structural divisions. By and large, they mirror and magnify longstanding dynamics, but they do so in ways that are much more visible than in traditionally unmediated settings. 


\section{Chapter 6: Power and Control}

When I asked Lolo and Gabriella, two Latina 15-year-old girls from Los Angeles, about their relationship with their parents, I provoked an explosion of pent-up frustration and exasperation. Gabriella was_once again_- "on punishment” after her mother caught her talking with her boyfriend around the corner from her house. This meant no cell phone, no MySpace, and no social life. Gabriella thinks her mother means well but she does not think she can live up to her mother's expectations_- "she wants me to be perfect ... hello!?” Gabriella does not feel close to her mother and Lolo is annoyed by how restricted Gabriella's mobility is. Of course, Lolo is not exactly pleased with her "nosy" and "paranoid" mother either. Lolo is annoyed that her parents always think she is up to "no good" when she goes online, because "that's what they see on the news." As Lolo and Gabriella map out their use of social media, they regularly return to the restrictions they face from their parents and how this affects them. Their parents' use of power and authority frustrates them:

Lolo: She always says this, "When you become a parent, you're gonna understand." She always says that.

Gabriella: Yeah, my mom always says that, too.

Lolo: I'm like, "No, I'm gonna somewhat remember my days. God, give me a little freedom.” 
Lolo and Gabriella think their parents do not understand them and they are infuriated by both the controls their parents exert and the condescending tone in which it is exerted. The frustration that Lolo and Gabriella feel is neither unique nor new. Teens' relationships with parents are often thought to be fraught and psychologists have extensively documented and theorized parent-teen conflict for decades (Montemayor 1983; Muuss 1995). Teens also have a long history of tension with other adults and authorities over issues of agency and control (Hine 1999; Savage 2007; Willis 1981).

Teenagers are alternately viewed by adult society as a nuisance who must be restricted or an impressionable population who must be protected; they are seen as both deviant and vulnerable (Buckingham 2000; Hine 1999; Sternheimer 2006; Valentine 2004). Many issues involving power and control stem from this dichotomous view of teens. Adults—parents, teachers, and other authorities—play a significant role in shaping the lives of teens and their views of teens often drive their actions. Adults can be powerful mentors and guides, but they can also be strict regulators and enforcers of adult policies. Personal and institutional constraints, lack of agency, and limited power can be tremendously frustrating (Csikszentmihalyi and Larson 1986) and teens struggle over whether to accept their social position or to challenge it.

The fundamental struggles for agency in an adult world have not changed much, but technology has introduced new inflection points for consideration. Social 
media operates simultaneously as a provocateur, an outlet, and a point of control. Depending on the dynamics in a given household or adult-teen relationship, networked publics can be a valuable space for intergenerational interactions, a respected youth-centered environment, or a source of tremendous conflict and fear. Teens can use social media to work through their struggles with parents and other authorities, but restrictions that extend into digital environments or a failure to understand technology's significance can rupture trust and destroy intergenerational relationships.

Social media has become a battleground for struggles between some adults and teens. Many teens turned to social media to escape control and yet parents and authorities followed them there. While some adults used these tools to enhance communication with teens, others perpetuated ongoing fears and called for heavy curtailment of teen participation, citing the dangers of online interaction and the importance of adult control over teen practices, spaces, and values. Media-produced fears turned into a widespread moral panic, centered on longstanding fears about inappropriate adult-teen relations, teen access to mature content, and teencontrolled social and sexual relations. The moral panic shaped how both adults and teens conceptualized these sites and, all too often, drove a wedge between them. By focusing on imagined dangers, often at the expense of actual risks, and blaming technology, fearful adults furthered generational divisions. 
Adult fear has made social network sites the locus of intense intergenerational struggles for agency and control. The underlying dynamics of these struggles are not new but nor are they historically stable. The rise of new technologies like social network sites invigorates debates about healthy engagement and showcases differences in parenting and educational philosophies. Many of the struggles stem from the need to balance control and freedom, especially with respect to public life, sociality, and sexuality. Efforts to maintain control reflect broader trends toward curtailing access and privacy in an attempt to create structure and prevent potential dangers. While most adults are well intended, not all teens welcome the resultant restrictions or believe that freedom must be surrendered for safety. In their efforts to achieve freedom, teens often resist adult control in ways that fracture intergenerational relations.

Adults who focus on their own teen years and emphasize a "right way" to grow up tend to further alienate teens, especially when the supposed right way does not take technology into consideration. "When I was your age" anecdotes are still used by adults to assert matured knowledge and authority, but, as teens are acutely aware, the technological infrastructure that shapes their social worlds was not part of teen life when their parents and teachers were children. Thus, when technology becomes a centerpiece for conversation between adults and teens, teens often tune out. For example, when white 17-year-old Julie from Kansas uses Facebook, her mother "makes fun" of her. This annoys Julie and she dismisses her mother by noting, "She doesn't understand it because she's old." By not understanding the ways in which 
technology—and particularly networked publics—is shaping teens' lives, adults reinforce the generation gap that divides them. Focusing on and failing to understand technology makes it difficult for adults to convey what they do know to teens.

This chapter analyzes some of the intergenerational struggles that have emerged in connection with social network sites. I examine how social media reinforces broader adult-teen relationships, in both beneficial and problematic ways. Intergenerational fault lines are not only reproduced online, but they are the source of broadly reaching fears that plagued the rise and adoption of networked publics. The circumstances around these fears and implementation of the fears as laws and regulations mirror the moral panic over public space (Valentine 2004). In moving between similarities and differences, I show the role that agency and control play in teens' efforts to engage with technology. Even when adults curtail mobility and privacy, teens respond by trying to assert authority in an environment that they relish as their space.

\subsection{Social and Structural Controls}

Today's teen culture is very much an extension of that which emerged in the twentieth and twenty-first centuries (Hine 1999; Savage 2007). Now, more than ever, teens are a constructed, controlled, regulated, commoditized, and segregated population. Adults continue to fear for and be afraid of teenagers, mostly because of the popular belief that adolescence and puberty render teenagers incapable of 
making rational decisions (Bradley 2003; Strauch 2003). Teens' lives are shaped by these beliefs, especially when adults seek to protect teens by heavily restricting what it is they can do and learn. As a result, as Stanley Cohen notes, "The young are consigned to a self-contained world with their own preoccupations, their entrance into adult status is frustrated, and they are rewarded for dependency" (Cohen 1972: 151). Teens are socially isolated for their safety and for the safety of adult society; child development is used to justify this policy, but little consideration is given to the costs of such restrictions and the ways in which limited access to broad social contexts curbs cognitive development.

Unlike other oppressed populations, teenagers are promised by society and its adult representatives that their status is temporary. Developmental stages are marked by age and teens' privileges can also be measured by age. Birthdates are celebrated as milestones, as they indicate new freedoms. Schooling is structured by age and those school years mark discrete steps toward graduation. Driver's licenses, tobacco and alcohol access, bar and club entry, voting rights, freedom from curfews, and other legal rights are all linked to age, although the ages themselves regarding some of these rights vary across the country. Age markers introduce rites of passage that provide legal, political, cultural, and societal relief. Even online, age is used as a delimiter for access. These factors combine to make age—and its symbols—highly desirable as indicators of status. While these age markers purportedly correlate with cognitive capabilities, historical work raises important questions about the true meanings of these age-driven rites of passage (Chudacoff 2007). 
While some teens accept the limitations afforded by their age and status, others desperately crave the perceived privileges afforded to adults. Adults generally believe that teens are not mature enough for adult vices like alcohol, sex, tobacco, loud noise, late nights, violence, or, for that matter, mobility and freedom. Engaging in adult behavior, or committing "status offenses" by seeking adult vices, before being "of age" is often believed to be a gateway to broader societal deviance (Mcnamara 2008). Status offenses can alternatively be viewed as a form of resistance to authority and a challenge to an oppressive age-organized society. Examining subcultures, sociologists have long argued that nonnormative behaviors and "deviance" are often a logical response to the social conditions in which teens live (Cohen 1972; Gelder and Thornton 1997; Hall and Jefferson 1976; Hebdige 1979). Supposedly deviant acts may also be teens' attempts to assert or attain power in a context where little is afforded to them. Rebellion may have more to do with a natural response to the curtailment of freedoms or the realization of a marginalized social position than a specific psychological stage (Gaines 1998; Willis 1981). My interviews with teens reveal that not much has changed in this regard. Teens still rebel, sneak out, and explicitly reject parental guidance. Adult vices like alcohol are still popular among teens, although studies suggest American teen substance abuse is declining (Johnston et al. 2007).

Even teens who do not rebel complain about those who hold power over themparents, teachers, authorities, and so on. The complaint lodged by Emily, a white 16year-old from Pennsylvania, that adults "don't give us enough credit" could have 
come from just about any teen in any town in the last century. Even in healthy environments, parents still try to pry information out of their children and teens still respond to adults' "What are you doing?" questions with "Nothing," just as they have for decades (Paul 1957). As teens move online, their desire for privacy extends there, as does their parents' desire to know what they are doing.

Another salient way in which control has been enacted is through systemic age segregation. Segregating youth from adults and splitting youth by age is a social project with a long history in American society (Chudacoff 1989; Hine 1999). Today, most teens have limited close personal ties to anyone but their peers and adults who hold power over them. The reasons behind why teens have such limited connections to other adults varied among those I interviewed, but they most commonly centered on limited opportunities, limited time, and fears surrounding potentially problematic adult-teen relations. Age segregation is not new, but it is more pronounced than ever in the lives of teens today. Furthermore, it is reproduced and reinforced online and the fears that drive age segregation are the source of moral panics surrounding environments in which adults may be able to interact with teens.

While the tensions between adults and teens are not new, the historical and social forces that frame youth culture continue to complicate intergenerational dynamics. Teens' relations to adults and adult society are quite varied, but popular beliefs and widespread attitudes create normative standards for teen behavior and 
intergenerational interactions. Even teens who generally have positive relations with adults are configured by these systems of power. Both positive and negative intergenerational connections are set against this cultural backdrop.

\subsection{Contemporary Adult-Teen Dynamics}

Teens' relationships with adults continue to be diverse and complex. For the most part, social media simply mirrored and magnified preexisting dynamics. Social media rarely damaged healthy intergenerational relationships and, in some promising situations, social media enhanced already open channels of communication. That said, unstable or contentious relations were sometimes weakened because of these tools, especially when teens turned to online environments to gain freedoms and adults responded by extending controls to the digital environment. Yet for the most part, the struggles that teens faced with respect to social media were a continuation of the struggles they faced offline.

\subsubsection{Household Dynamics}

Parent-teen connections are the most significant intergenerational relationships in teens' lives. Not all teens have healthy relationships with their parents, but many of the teens I interviewed liked, trusted, or at least respected their parents. Household dynamics are a salient force in teens' lives and the factors that configure their households often shape their online engagement. 
Kevin, a white 15-year-old from Seattle, told me that he gets along well with his parents. He believes they trust him and, in return, he trusts them. "I can communicate to them well because I don't have anything to hide. "He attributes the open communication channels to being the third son, noting that his parents learned from dealing with his older brothers. Kevin says that he has tremendous freedom to do as he pleases so long as he obeys the law. While there are things he does not tell them-like "late-night escapades"-he is conscious to stay out of trouble because he does not want to disappoint them. His freedoms extend to social media and he feels free to make his own choices online. Kevin says that his parents respect his privacy, both online and offline.

In the same way but in the opposite situation, offline restrictions extend online for Anindita, an Indian 17-year-old from Los Angeles. She is not allowed to go out and her mother monitors her online activities. When Anindita began dating a boy of a different race, her father made her choose between the boy and her family. Although some teens may have chosen otherwise, Anindita decided to dumpher boyfriend. She believed her father was simply looking out for her and she trusted him to guide her. In the same way, she is cautious about her online interactions with boys because she knows her parents are watching. Anindita's mother plays a central role in her life, both as a regulator and a confidante.

“My mom's like my best friend. I tell her everything. She knows everything. She's the one I gossip with. She likes it like that. She's like, 'It's better to keep it like 
that 'cause then you don't create enemies.' That's what I do. I talk to my mom. She's my best friend."

Although Anindita has no privacy or mobility because her parents do not trust her, Anindita sees her relationship with her parents as positive. She respects their rules and believes that her parents are wisely looking out for her better interests.

In both of these examples, the rules and norms that structure the household extend online. Because Kevin and Anindita respect their parents' approaches and meet their expectations, there is little conflict. While most teens reject the notion that parents should be on social network sites, such healthy relationships can ease the intergenerational divide around social network sites. In some cases, as with Anindita, teens accept their parents' viewing their profiles as a protective measure. In others, as with Kevin, parents have accepted social network sites as teen space and trust their children to act wisely. Much more rare are teens whose parents create accounts and join them on social network sites.

In Los Angeles, twin 16-year-old brothers Denzel and Nick regularly communicate with their father through MySpace. ${ }^{10}$ They both play football at school and their father is their football coach. Thus, when he joined MySpace, he immediately connected to Denzel, Nick, and all of their friends. The twins like that their father is on MySpace, although they find his sense of humor questionable.

\footnotetext{
${ }^{10}$ Denzel self-identifies as black; his brother Nick self-identifies as black with Native American roots.
} 
Denzel explains that his father will regularly leave comments on his profile, saying things like, “'You guys pages are funny. They're whack.' And he'll be, 'All right. I'm gonna see you all later.' And he'll say, 'Guess what? I'm gonna take your phone.' And then he'll be, 'Laugh out loud. I'm just playing.'” While the boys find their father's humor odd, they are entertained that he managed to get so many Friends because he threatened push-ups for the whole football team if their friends did not accept his Friend requests. More than anything, Denzel was relieved when his dad joined MySpace because this eased earlier tensions that occurred when he did not approve of their participation on the site.

Parent-teen communication on social network sites can enhance already healthy relationships by introducing new moments of dialogue. In Los Angeles, I talked with a father of a 16-year-old girl who was overjoyed when his daughter invited him to join her on MySpace. He saw this as a signal that he was doing something right as a parent. Through MySpace, he was able to learn things about her tastes, interests, and priorities that he did not know. At the same time, he struggled to make sense of signals that worried him. Upset by a personality quiz that suggested drug use, he carefully confronted her; she helped put the reference into context and reassured him that she had no interest in drugs. Always nervous about approaching this topic because of his own past, he valued the way that MySpace eased his ability to do so. Although father and daughter were already close, MySpace further strengthened their relationship and gave them a new channel with which to joke with one another. 
Even when teens have healthy relationships with their parents, not many teens want to interact with their parents through these sites and parental participation can be unsettling. Aria, a 20-year-old college student in California, blogged her reaction to her father's decision to join Facebook. She was initially uncomfortable because she did not believe that Facebook "is something you can do with someone whose genetic material you inherited without subverting the laws of nature." Yet she did not think she had a choice, noting, "I may not be in the running for Golden Child, but I sure as hell enjoy not paying my own tuition." For this reason, she accepted his participation and began enjoying his efforts at creating a "patently hilarious" profile. Aria continued by reflecting on the "mind-boggling generational role reversal" of parent participation in youth space. "How strange it is to watch our parents learn things we learned first! Is this the feeling they get when we get our first paycheck or bring home boyfriends or start driving?" Even though Aria was initially wary, her relationship with her father was strong and she ended up being more humored by his participation than upset.

Unfortunately, not all teens have healthy relationships with their parents. At the most basic level, there can be a lack of trust or resentment, often stemming from struggles over control. For example, Ana-Garcia, a Guatemalan-Pakistani 15-yearold in Los Angeles, told me that her father is extremely "overprotective" and "controlling," especially in relation to her, her sister, and her mother-"my brothers get to go out with their friends sometimes, but me and my sister, we're just stuck." While she is not allowed to leave the house and has an unmanageable number of 
chores, her brothers have no responsibilities and may do as they please. On weekends, when her father is not around, her mother will sometimes let her walk to the nearby store even though both know that this is forbidden. Yet most of the time, Ana-Garcia's mother does nothing to challenge the rules dictated by her father and Ana-Garcia finds this upsetting. To explain the reasoning behind her father's rules, Ana-Garcia simply says that it is "cause he's Muslim." Ana-Garcia says that the restrictions get worse as she grows older because her father believes that she is more likely to have inappropriate desires as she gets older. Ana-Garcia lacks mobility, privacy, and control and she resents this. Going online is her only outlet, but the household computer belongs to her brother and she is lucky if she can get online for 30 minutes a day. While access is an issue, privacy is a bigger problem because her parents monitor her and her brothers often compromise her accounts. Recently, her younger brother found her MySpace profile and deleted most of her Friends. Noting that little is fair at home, she says that she simply must ignore her brothers because reporting on their misdeeds only makes things worse. While Ana-Garcia accepts the power dynamic in her household, she says she has lost any sense of trust in other people. She told me that she has closed down and does not want to share anything with anyone. ${ }^{11}$

While Ana-Garcia's relationship with her parents is sour, there are more extreme examples of unhealthy parent-teen relations. In Iowa, white 18-year-old Wolf told

\footnotetext{
${ }^{11}$ Given this, I was surprised that she was willing to talk to me. Yet, she seemed both authentic and resigned to a home dynamic in which she felt she lacked control.
} 
me that he started sleeping on friends' couches after he turned 18 to avoid the ongoing alcohol-driven fistfights with his father. Wolf is visibly out of control, angry, and confused, and the only interactions he has with adults are when he gets into trouble at school or with the law. Wolf regularly looks for trouble and, most likely, attention. Wolf says that he works out his stress by punching people and "If I've had a bad day particularly and I'm feeling depressed I'll drink myself into the most insane stupor I can and wake up in the toilet the next day. 'Cause ... I would be vented the next day. I wouldn't have a hangover and I'd just be, 'All right, cool. New day."”

While Wolf can do whatever he wants online, he rarely spends time there. He would rather interact with people in person to be able to "gauge their reactions." He spent most of the interview trying to provoke me while his white 18-year-old friend Stan mocked Wolf's hard edge.

Like Wolf, Ty, a black 17-year-old in Los Angeles, has struggled in relating to his parents. He left his mother's house in Philadelphia to get away from an unhealthy home and social environment. He moved in with his dad in Los Angeles, but their interactions turned violent and Ty was kicked out. He ended up as a ward of the state in a group home, where he was able to do some thinking. Eventually, Ty reached out to his father and they have been rebuilding their relationship through their mutual interest in dancing. Ty's relationship with his father works because his father no longer holds power over him. While his relationship to his father has 
improved, Ty's life is structured by the state and his freedoms are curtailed by the group home in which he lives. He must do explicit chores and follow strict curfews. His social opportunities are heavily curtailed; Internet access is unavailable and phone time is limited. That said, the group home does not monitor his online activities. Ty goes online at school and on the rare occasions when he can obtain a pass to visit a friend's house. MySpace is extremely important to him—he uses it to share his writing and the music that he made. He also uses it to flirt, catch up with friends, and connect to extended family members. For Ty, MySpace provides opportunities that otherwise are strictly curtailed because of his living situation.

Unfortunately, the physical and emotional absence of parents occurs all too frequently and takes a toll on teens. While teens resent being controlled by their parents, they also resent being in control of their parents. Alexis, a Latina-white 16year-old in Seattle, takes care of her little brother and cleans up after her mother because her mother's use of crystal meth has made her "kinda gone." At home, Alexis has many responsibilities, but online, she is able to do as she pleases. She enjoys flirting and being provocative, engaging in behaviors that might be seen as risky. MySpace is an outlet for Alexis, a place where she can have fun.

Teens who have healthy relationships with their parents are frequently open about social network sites. Whether they participate or not, these parents tend to know about social network sites and have an opinion of them. How strict they are varies and restrictions can hamper the relationship. Conversely, in less healthy 
households, parents are often unaware of their child's participation on these sites or social network sites are an additional point of contention. While most teens use social network sites to socialize with their friends, I found that teens in more controlling or tumultuous households were more likely to use them as an escape. Other scholars have found that poor home environments full of conflict and poor parent-child relationships are correlated with a host of unhealthy online practices, included online harassment and sexual risks (Wolak et al. 2003; Ybarra and Mitchell 2004). Yet in both positive and negative settings, social network site engagement is shaped by the parent-teen dynamics.

\subsubsection{Engaging with Teachers, Youth Pastors, and Other Trusted Adults}

Parents are not the only important adults in teens' lives. Age segregation and structural forces limit teens' interactions with most adults, but there are still adults who play a significant role. In my fieldwork, the most visible of these adults were teachers, extended family members, youth pastors, and friends' parents. For example, Summer, a white 15-year-old from Michigan, is especially close to her friend's mother, who is "like a second mother to me." Summer feels safe turning to her about issues that she does not feel comfortable discussing with her own mother. “There are things she knows that my mom doesn't know just because, I don't know, I feel more comfortable telling her and my best friend than I do my own mom." Summer often goes over to her friend's house to seek advice from her friend's 
mother. This relationship is important to Summer because there are few other adults whom she feels safe approaching about her doubts and concerns.

In some cases, teens who have positive relationships with trusted adults use social media to connect with them. Most adult-teen online interactions are an extension of the offline relationships. Teens talked about communicating with aunts and uncles between holidays, seeking advice from teachers, and keeping up with religious youth groups through social media. By and large, the media tends to focus on negative adult-teen online interactions (Gordon 2006; Hempel 2005; Schrade 2006), but the vast majority of adult-teen interactions online are productive and healthy, opening channels of communication so that teens can enhance connections with adults they know and respect to get advice and support.

Mediated exchanges between teachers and students can allow teens to obtain personal and academic support, as well as information and answers to questions. For example, Sara, a white 16-year-old from Austin, Texas, told me that one of her teachers makes his IM screen name available to students "and he'll get on every night before a test, in case you have questions on what the test is about." While Sara does not always ask him questions, his willingness to answer them makes her feel more confident as she is studying.

Teachers also find this opportunity to be quite valuable, especially when it comes to helping struggling teens, those who are too embarrassed or crunched for time to approach teachers during the day, and those who are in need of general support and 
validation. For example, Mr. C, a high-school math teacher at a charter school in a poor district of the Bay Area in California, told me that MySpace allowed him to connect with students and build bonds of trust that he could leverage in the classroom. Mr. C initially created a MySpace profile for personal purposes, but as teens began flocking to the site, he reworked his profile to be teacher-centric, stating his identity as a math teacher clearly in his "About Me." He wrote a handful of blog entries, including "Calculus Blog" and "SAT Prep." As a young teacher in a failing school, Mr. C struggled to find ways to motivate his students to learn; MySpace provided a valuable channel for him to connect with students.

As Mr. C's students and former students found his profile, they began commenting, leaving updates, and asking questions. For example, one male former student writes, "just comin' to say hello and that I really do miss your math teaching!" When I asked him about commenting, Mr. C told me that he regularly responds to students' messages and that he believes participating on MySpace can be tremendously helpful to his students, especially those who are struggling. In one encounter, a female student left a message asking Mr. C to "pleeeeeeeeeeease tell me why pre-calculus is important to me and how it will benefit me and affect me after I graduate? There are sooooooooooo many adults who don't know pre-calculus and they are doing quite well in life." Recognizing this comment as an ideal opportunity for intervention, Mr. C posted a comment on her profile in response, explaining that she will probably not use pre-calculus, Shakespeare, or iambic pentameter as an adult, but that "studying these things ... helps you get good at learning how to learn. 
And that, you will definitely have to do for the rest of your life. That's practically all we do as adults." He continues to explain how learning math helps with problem solving, pattern recognition, and understanding how the world fits together. "That may not be the most satisfying answer, but it's the truth!"

Teachers are not the only adults who believe they can benefit from interacting with teens online as well as offline. After being initially horrified by MySpace, Ben, a youth pastor in Atlanta, quickly learned that social media allowed him to connect with his young parishioners in new ways. He joined the site out of fear that the teens he worked with might be in danger, but he now sees social network sites as a key tool for his work. He is Friends with all of his students and sends Friend requests to newcomers to make that initial connection. He sends bulletins on MySpace and leverages Facebook Apps to goof around with teens. As he explains, "The connections may not be as 'deep' as face-to-face conversations but they're connections nonetheless, which is important in this line of work." For Ben, tools like social network sites allow youth pastors to play more of a role in teens' lives. As he explains, "The tendency of youth pastors is to only have meaningful contact with students once or twice a week (on Sundays and Wednesdays). SNSs provide a fairly effective means to at least keep lines of communication open between the pastor and his/her students."

While Mr. C and Ben are exceptional in that they are willing to go out of their way to meet teens on the teens' turf, most adults who work with youth are opposed 
to these sites. Countless news stories tell of teens getting into trouble when teachers and authorities find their profiles (Kaufman 2006; Kornblum and Marklein 2006), and social network sites have further complicated already rocky relationships between teens and teachers. In Seattle, white 18-year-old Samantha told me about students in her class who were suspended because of fake teacher profiles that they created. In some of the profiles, teachers were identified as gay and in love with each other. Other profiles constructed teachers as pedophiles. While the students viewed these profiles as jokes, the school administration did not find them humorous; the students were suspended. In this way, the schools asserted control over these spaces.

Because of such incidents, many teens hesitate to interact with adults through these sites. Penelope, a white 15-year-old from Nebraska, created a Facebook group around her geosciences teacher. He makes pithy statements that she and her friends found entertaining so they started posting them to a group called "I failed geoscience but I aced life." After someone at school showed this group to him, Penelope and her friends were terrified that they would get into trouble.

"We didn't want him to see that 'cause you know we might get in trouble, but then he saw it and he was just like, "Well I'm going to report this,' then he was cool with it. I mean I thought we were going to get in trouble but we didn't."

This incident made Penelope extremely cautious when involving teachers. Later, when she decided to post a picture of her favorite teacher to her profile, she asked 
permission first so that the teacher would understand that she wanted to honor her teacher, not inadvertently mock her.

In all of these cases, the issue is one of trust and control. Teens are willing to interact with adults with whom they have healthy relationships through social network sites when they believe these adults respect the space and the ways in which teens are engaging. When teens do not trust adults offline, they do not trust them online. Their trust is also fractured by adults' attempts to assert control over the space and the ways in which teens may participate.

\subsection{Fears and Moral Panics}

Struggles between teens and adults over social network sites were further complicated after a moral panic emerged. Centered on the dangers of interactions between adults and minors on MySpace, this moral panic damaged intergenerational dynamics in two ways. First, it prompted parents and schools to restrict access and increase online surveillance of teen participation. Second, it discouraged teens from interacting with any adult online, further solidifying online age segregation. The moral panic surrounding MySpace parallels earlier moral panics and is driven by unwarranted fears. Nonetheless, the effects of these fears were significant.

Fear has been at the heart of adult concerns about teens for decades (Cohen 1972; Goode and Ben-Yehuda 1994; Springhall 1998). Adults are simultaneously 
fearful for and afraid of teens and youth culture (Buckingham 2000; Valentine 2004). Fears are culturally dependent, but in the United States, violence, drugs, and gangs are all used as proof of teens as miscreants while pedophiles, abductions, and peer pressure showcase teen vulnerabilities. The rise of mass media has only amplified these concerns, generating a culture of fear that is outright pervasive (Glassner 1999). The operationalization of fear is one of the most significant ways in which adults have leveraged power to control teens.

While everyday fears are used to restrict teens in different ways, fear takes on a different form when it becomes a tool for the construction and perpetuation of a moral panic. In his seminal text on moral panics, Stanley Cohen (1972: 1) defines a moral panic as:

"A condition, episode, person or group of persons emerges to become defined as a threat to societal values and interests; its nature is presented in a stylized and stereotypical fashion by the mass media; the moral barricades are manned by editors, bishops, politicians and other right-thinking people; socially accredited experts pronounce their diagnoses and solutions; ways of coping are evolved or (more often) resorted to; the condition then disappears, submerges or deteriorates and becomes more visible. ... Sometimes the panic passes over and is forgotten, except in folklore and collective memory; at other times it has more serious and long-lasting repercussions and might produce such changes as those in legal and social policy or even the way the society conceives itself." 
Moral panics are epitomized by mass hysteria and collective delusion created from a disproportionate amplification of rare events or myths (Goode and BenYehuda 1994), the construction of scenarios that substitute for facts (Glassner 1999), and reinforcement by terror talk that capitalizes on people's fears (Valentine 2004). Most moral panics target youth and youth culture. Moral panics have surrounded every youth-valued media, from penny theaters and comics to rock and rap music (Springhall 1998). Not surprisingly, when teen engagement in the Internet gained visibility in the 1990s, a moral panic erupted over the potential dangers youth faced online, most notably the dangers of child predators. While many fears of specific media tend to fade through time, the general fears about child safety, teen sexuality, predatory adults, and child abduction are cyclical. The moral panic that erupted over MySpace stemmed from these same concerns.

\subsubsection{The MySpace Moral Panic}

After MySpace gained popularity among American teens, fears spread about the potential dangers that they faced in this new medium. Fears about sexual predators were amplified after the Dateline TV show “To Catch a Predator” began using fraudulent profiles on MySpace to entice potential pedophiles into real-world encounters with "children" and to document their reactions when they learned that they had been duped. This and other forms of "perverted justice" were used to showcase the "dangers" of social network sites. To further complicate matters, countless print and broadcast stories were dedicated to the potential dangers of 
MySpace (Bahney 2006; Hempel 2005; Pimentel 2006). Combined, the portrait created by these different media sparked a moral panic.

Media coverage of MySpace focused on the hypothetical dangers the site presented, but the coverage was often misleading or inaccurate. For example, after two girls in California disappeared, the media ran a series of articles on how they were abducted because of their MySpace profiles (KNBC 2006). I followed the case closely, talking to law enforcement and MySpace. I was scheduled to appear on TV alongside the girls' parents to debate the dangers of MySpace when police officers found the girls; they had run away. The media did not amend the stories or issue corrections or conclusions.

Public services and child safety organizations further fueled this moral panic by misappropriating scholarly research to exaggerate the dangers teens faced. Many referred to the Online Victimization studies done at the Crimes Against Children's Research Center (Finkelhor et al. 2000; Wolak et al. 2006). These organizations regularly pronounced that between one in five and one in seven minors are sexually solicited online. The Ad Council used this data to run advertisements stating that children are solicited every 10 minutes. While these numbers do come from these studies, they do not mean what the public believes them to mean. First, "online solicitation" in this study is defined broadly to include sexual harassment and flirting. Second, an astonishing 43 percent to 63 percent of those doing the solicitations are other minors, while only 1 percent to 9 percent of those soliciting 
are over the age of 25 (Finkelhor et al. 2000; Wolak et al. 2006). Finally, because virtually no teen responds to sexual solicitations from strangers, these rare incidents amount to little more than unsolicited spam. Yet, this data was leveraged to propagate widespread fears on TV, in school assemblies, and in parenting events.

As with other moral panics, the one concerning MySpace had more to do with perception than reality (Marwick 2008). As researchers began investigating the risks that teens faced in social network sites, it became clear that the myths and realities of risk were completely disconnected (Wolak et al. 2008; Ybarra and Mitchell 2008). Counter to the highly publicized myths, most problematic sexual interactions between adults and minors involving the Internet resemble statutory rape in that teens knowingly and repeatedly met with adults for sexual encounters (Wolak et al. 2008). Most encounters did not involve deception, forcible sexual assault, or pedophilic child molesting; the victims were usually teenagers (not children) and the perpetrators were mostly men in their 20s (Wolak et al. 2008). This is not to say that these encounters are not problematic; they are. It is just that the public image being presented is inaccurate. Furthermore, Internet sexual solicitation is down since the original study in 2000 (Wolak et al. 2006), and social network sites have not increased the risk of victimization (Wolak et al. 2008).

While statistical data makes it clear that the panic around social network sites is groundless, the fear was nonetheless real. Fears prompted adults to seek efforts to control teens' engagement with these sites. At a legislative level, lawmakers began 
introducing bills to try to block teens from using school or library computers to access any genre of social media that allows interactions between strangers (Fitzpatrick 2006; Stevens 2007). These bills have not passed, but others continue to emerge. Even without these bills, schools began blocking social network sites. At home, parents began limiting and monitoring teen access.

The moral panic surrounding social network sites combined elements of previous panics concerning new genres of media and those involving teens and public spaces. Little is new about the moral panic around social network sites and networked publics. Yet historical insignificance does not make the impact any less powerful. This panic constructed and configured how teens saw social network sites and how they interacted with them.

\subsubsection{Teen Responses to a Culture of Fear}

The discussions of predators on MySpace were pervasive in youth culture and they shaped teens' ideas of and experiences with the site. Fear configured teens' participation and, as such, it was an element of power itself. Some teens' parents forbid them to use the site because of the risk of sexual predators, while other teens tried to keep the stories of predators from reaching their parents for fear of being restricted. Sara, the 16-year-old from Austin, is forbidden to create a Facebook because her mother worries about sexual predators. She never created an account, but she does browse Facebook when her friends log in to the site with their accounts. She does not share her mother's concerns, but she does think that there are dangers 
to having a profile-she heard that some teens were rejected from college because of their profiles. While Sara respects her mother's restrictions, other teens ignore such restrictions and create an account behind their parents' backs. Natalie, a white 15year-old in Seattle, has a MySpace against her mother's wishes. She says that her mother is concerned that she will be raped, but she thinks her mother's fears are unfounded. By taking active steps to be safe and not talk to strangers, she thinks that she has the right to have a profile. Yet even though she feels justified, she worries that her mother might find out.

In addition to the news, teens regularly faced school safety assemblies concerning predators. While some teens rejected these messages as unfounded, others embodied the fears the adults presented. Sabrina, a white 14-year-old from Texas, does not communicate with anyone in public online communities because "any person could be a 40-year-old man waiting to come and rape me or something. I'm really meticulous about that, because I've heard basically my whole life, don't talk to people you don't know online, 'cause they'll come kill you." She has never known anyone personally who has been contacted by a predator, but she has seen episodes of Law and Order in which terrible things have happened to people who talk to strangers online. For a long time, she was afraid to get on MySpace for fear that stalkers might find her. Her friends convinced her to join by pointing out that she could protect herself. Still, she worries that she might be stalked. "It's still like a possibility, because I mean anyone can just pick, click on your profile and find out kind of what's going on." Sabrina's experience with social media is shaped by 
pervasive stranger-danger rhetoric. She is cautious in her decisions, but she is constantly afraid that something will go wrong. Sabrina's fears limited her participation in MySpace and other online communities because she was afraid that she might bond with people who would turn out to be stalkers.

Many teens, especially girls, fear the possibility of being raped, stalked, kidnapped, or assaulted by strangers as a result of their participation online. Their fears are not rooted in personal experience but in media coverage, parental concerns, and poor risk assessment. No teen I interviewed knew anyone who had been a victim of an online pedophile, but many referred to Dateline's "To Catch a Predator" as proof that predation is real.

Fear also drives teens to engage in some places but not others. Because the concern about predators is primarily targeted at MySpace, some teens choose to create profiles elsewhere for safety. For example, Julie, the 17-year-old from Kansas, is not allowed to have a MySpace because her mother heard terrible stories in the media, but because her mother never heard about bad things happening on Facebook, she was allowed to create a profile there. She acknowledges that her mother does not understand Facebook. Many of the teens I interviewed who were only on Facebook shared Julie's belief that Facebook was safer, even if they were not quite sure why. As Vietnamese 16-year-old Tara from Michigan explains, "It kind of seemed safer, but I don't know like what would make it safer, like what main thing. But like, I don't know, it just seems like everything that people say, it seems safer." 
Because Tara regularly heard that Facebook was safer than MySpace, she believed it, even though she had no idea what made it safer or if it actually were.

Notions of fear and safety are often disseminated through communities as rumor; often, people's perceptions of risk and safety do not align with what the research shows are risky behaviors or appropriate steps for safety. That said, many teens I interviewed questioned the messages they were receiving. Few teens outright ignore the various discussions of risk, but many believed that the news reports about the dangers of MySpace were sensationalistic and rooted in a lack of understanding of social network sites. While most believe that there are indeed risks to going online, they believe that the news coverage portrays an unrealistic image of what is happening, capitalizing on adults' failure to understand social network sites and their underlying lack of trust in their children and for their children's safety.

Often, teenagers told me that only "stupid" people would ever respond to messages from strangers. At an after-school program one day, a girl adamantly told me that there is one correct response to creepy Friend requests and messages: "You just delete that shit.” Teens argue that they should not be judged poorly and controlled because some teen out there did not learn basic safety lessons.

Many teens believe they have a better understanding of how to be safe on these sites than their parents do. For example, Ann, a white 15-year-old from Seattle, complains that her parents, like most parents, "do not understand what protection some MySpace sites have-like no name and address, and you are able to restrict 
yourself to people you know only." She keeps her profile private to reduce her parents' worries. She knows that strangers are on MySpace, but this does not concern her. On the rare occasions when she and her friends receive messages from strangers, "we just tell them to bug off and they usually do." For Ann, the opportunities presented by MySpace far outweigh the potential dangers and she works hard to help her parents understand this.

Teens had numerous strategies for staying safe, many of which mirrored the strategies that adults and safety organizations perpetuated. Upset that teens were being restricted from social network sites, Danny, a 17-year-old from Florida, emailed me with a list of safety guidelines that he thought all teens should adopt:

A) Only accept "Buddy Requests" from people you know personally/are bands. (Cheesy, I know, but it's the safest way. I have around fifty-eight friends, and I can tell you their name, address, or what their most popular songs are).

B) Don't give out personal information. (I don't have my school listed even! State and City, that's it. Don't give it when asked.)

C) When posting a picture, ask if it shows you in a positive manner, and if it is something you would wear to go visit your grandparents/religious leader. (Mine are!)

D) Keep your parents involved. If you hide things from them, they will find out, and you will get in trouble. Be open with them-most of the time, they are cool about stuff like this. 
Another approach that many teens take is to keep their profiles private or Friends-only. For most teens, these sites are about interacting with people they already know, and friends-only Friend lists, private profiles, and limited identifying information make complete sense. Catalina, a white 15-year-old from Austin, is not concerned about predators "because I know that I don't talk to anyone I don't know and I don't go looking for someone that I don't know." Like many of her peers, she believes that making her profile private and talking to and connecting only with friends are good strategies for avoiding dangerous strangers. To prevent stalkers or abductors, other teens argue that it is important to not publicly post any identifying information-name, city, phone number, IM screen name, and so on. Heather, a white 16-year-old from Iowa, says that she does not indicate her school name or grade because she believes that this would allow someone to track her down. Some teens argue that first names are all right, but last names are not.

Teens' sense of what it means to be safe online is very much driven by adult education programs. Online safety programs and law enforcement have been encouraging teens to limit identifying information for more than a decade. Private profiles and peer-only social interactions have also been core messages in educating teens about safety and social network sites. While the teens I interviewed had nothing positive to say about the school assemblies and online safety programs that they were given, it was also clear that they had integrated the messages contained in these programs into their practices. 
While these safety measures have merit and teens should be commended for taking the issue into their own hands, their approaches reproduce the inaccurate account of online risks that adults hold and it is not clear that these steps actually prevent real dangers. Research shows that teens are not randomly at risk; psychosocial problems combined with risky behaviors are the key predictors of risk (Ybarra and Mitchell 2008). In other words, the teens others see as "stupid" for not understanding how to be safe may actually be dealing with much larger personal issues. Many of these approaches assume that deception is at the crux of predatory adults, when this is rarely the case (Wolak et al. 2008). Because of this, personal information alone does not put teens at risk for sexual solicitation (Wolak et al. 2008), even though not providing identifying information is touted as the first step that everyone should take.

While teens' safety solutions may have no real effect on preventing sexual solicitation, they do have a secondary desirable benefit because identifying information does put teens at risk of being observed by prying college admissions officers, school officials, law enforcement, and other adults who hold power over them. Or, as Wolf, the 18-year-old from Iowa, explains, providing identifying information online is foolish "because it'll bite you in the ass some way or another. There's 1,000 different way it'll happen." Wolf is not concerned about predators, but he can think of many people he would rather not interact with online. If efforts to ward off potential predators result in warding off other types of unwanted contact or observation, teens are more than willing to take necessary safety precautious. 
In adopting safety strategies, teens worked to address the narrative of the moral panic, failing to realize that was not the logic behind it. Many teens grew frustrated that their safe practices did not stop adults from panicking. Some grew angry at the media's negative portrayals; others were upset by their parents' lack of understanding. James, a white 17-year-old with Native American roots from Seattle, was additionally annoyed by the bipolar approach that the media tended to take. He thought that it was odd to see "MySpace Is Bad" articles alongside "MySpace Is Good" stories. Those focused on predators and rapists proclaimed the former while those that detailed how missing people were found argued the latter. More than anything, the extremes bothered him because it felt so hypocritical- "it's kinda like a two-face thing, you know?" James's frustration stems from his realization that the way the media portrayed MySpace shaped public sentiment. James sensed and did not like the manipulative role that the media played. Yet, even recognizing this, he took many of the same safety precautions as his peers. He could not be sure, but he did not want to find out.

The moral panic surrounding social network sites fundamentally altered teens' relationship to these sites and to the Internet more generally. It prevented some from participating and it shaped others' practices. Neither the teens who took the fears seriously nor those who rejected them as sensationalistic could avoid the culture of fear. Even those who were free to participate had friends who were not. The companies behind the sites changed features to address public concerns and this affected teens' experiences with the sites. Additionally, teens always had to account 
for their fears in justifying their practices to adults. Moral panics assert control by tapping into underlying fears.

In her analysis of children's access to public spaces, Gill Valentine (2004: 27) argues that "by reproducing a misleading message about the geography of danger, stranger-danger educational campaigns contribute towards producing public space as 'naturally' or 'normally' an adult space where children are at risk from 'deviant' others." In other words, moral panics and the responses that emerged to address the potential risks configure youth practices in restrictive ways. Moral panics work by creating a culture that restricts itself more than any piece of legislation could possibly do. In their attempts to redress adult concerns, teens limited their own access to public space. While teens are seeking to connect with peers, there are costs to their lack of engagement in public space.

\subsection{Access, Privacy, and Control}

By most measures, teens are safer now than they have been in decades; crimes against children have gone down, but media coverage of dangers has gone up (Valentine 2004). Thus society perceives that today's teens are more at risk than ever before and has a constant sense that every new technology brings about new dangers. This "culture of fear" is driven by a perception that is completely misaligned with real risks and dangers (Glassner 1999). 
Concerned adults often seek to control teens' freedoms in an effort to keep them out of danger. Moral panics heighten adults' desire to control situations that teens might face. While moral panics emerge out of concern for youth, they often result in a decline in youth freedoms in which teens lose access to publics and the right to privacy. Mobility and privacy are limited in both explicit and implicit ways; restrictions are enacted through law, social norms, and in individual households. Many of the parents who I met believed that responsible parenting involved taking fears seriously and responding by limiting mobility and constantly supervising teens' activities. These restrictions are often the source of youth anger and frustration.

Throughout the 1980s and 1990s, fears about the dangers of public spaces prompted parents, legislators, and other adults to curb teens' access to public spaces (Valentine 2004). As a result, many of the teens I met had very limited access to public spaces. Often, they turned to networked publics as a substitute for inaccessible physical environments. Lila, a Vietnamese 18-year-old from Michigan, was adamant that she would rather hang out with friends in person "for sure ... but if you don't have the option, then you can just go online." For many teens, networked publics became a refuge in response to the curtailment of offline mobility. Yet when the moral panic erupted over MySpace, teens were faced with similar restrictions extended to the digital environment. Once again, teens found their freedoms of mobility and privacy curtailed. But because of differences between networked publics and physical public spaces, the restrictions played out differently. 


\subsubsection{Restricting Access and Mobility}

Fears surrounding the dangers of specific spaces—-physical and digital—often prompt adults to curtail access to those spaces in order to protect youth or limit their ability to cause trouble. These restrictions come in different forms and are enacted by both parents and authorities.

Legal restrictions are one technique that adults take. In response to fears over teen activities in public spaces (Valentine 2004), curfew laws were enacted to address gangs, delinquency, teen violence (Ruefle and Reynolds 1995), and truancy (Cochran 1997). To further expunge teens from public spaces, cities and towns also introduced antiloitering laws that restricted where teens could hang out and for how long, although some were declared unconstitutional (1999). While research shows that curfew laws have little, if any, effect (Adams 2003; Males and Macallair 1999; McDowal et al. 2000), no curfew laws have been repealed. When the mayor of New Orleans was asked to justify teen curfew laws in the face of such research, he stated, "It keeps teenagers off the streets. They need it, there's too many teenagers hanging around the streets" (Valentine 2004: 91). Fear prompted legislators to limit teens' access to public spaces, but desire to assert authority and control teen freedoms allowed the restrictions to continue.

Similarly, legislative attempts have attempted to restrict teen access to online environments. Early efforts by Congress to limit minors from accessing websites with “obscene or indecent content" (Communications Decency Act of 1996) or 
content that is "harmful to minors" (Child Online Protection Act of 1998) have been curtailed by judicial decisions declaring them fully or partially unconstitutional. That said, one piece of legislation intended to limit marketing material (Children's Online Privacy Protection Act of 1998) has inadvertently limited access to minors under the age of 13. More recent bills have sought to ban minors' accessing from schools and libraries any social network sites and websites that enable communication (Fitzpatrick 2006; Stevens 2007). These attempts have failed but more legal efforts are under way.

Legal efforts to limit when, where, and how teens may engage with publics are the most formal means of controlling access and mobility, but they are not nearly as successful as parental restrictions. Media-produced horror stories about youth in public spaces prompted many parents to limit teens' physical mobility. Parents use fear in the process of regulating mobility, teaching their children to fear while simultaneously mandating rules based on their own fears. In some households, parents choose to create strict structures around their children's lives such that children lack free time in which to seek out publics.

Although the moral panic around public spaces is almost 20 years old, many teens and their parents still believe that public spaces are dangerous. Jordan, a Mexican-white 15-year-old living in Austin, told me that she is not allowed to go outside because "my mom's from Mexico ... and she thinks I'll get kidnapped." Natalie, the 15-year-old in Seattle, told me that she understands why her parents do 
not allow her to walk anywhere, but she wishes that the world were not so dangerous. Both teens and parents told me that they believed that the world was more dangerous today than ever before.

For most teens, the perceived dangers far exceed the actual risks. In Iowa, white 17-year-old Sam was afraid to ride his bike at night because "you never know who's out there late at night. I mean this isn't like crime capital of the world, but there is weirdoes here like there is everywhere else." While Sam is genuinely afraid, there are few crimes in his town and the risks are low. Some communities do have real and present dangers. For example, in a gang-ridden section of Los Angeles, I met black 16-year-old Keke only a few weeks after her brother was murdered by "Mexicans [who] thought he was a gangbanger." Keke has every reason to be afraid, but she is mostly frustrated. Her mother is not strict, but "she would rather me stay at home, because I can't go nowhere because of the gangs." Keke still goes out but she is very careful about where she goes and irritated that she has to take this into consideration. "I hate not being able to go places. I hate having to be careful what color shoes I'm wearing or what color is in my pants or what color's in my hair. I hate having to tuck my chain in when we walk in through a crowd." While Keke has a much higher likelihood of running into real danger than most teens I met, she also has fewer limitations to her mobility than those whose communities are less dangerous and more fearful. 
In speaking with parents, I found that many-especially those from wealthier and less crime-ridden communities-believed that they restricted their kids' mobility more than their parents had restricted theirs. They argued that these restrictions were necessary because they believed that society was becoming increasingly dangerous. Many of the teens I met never played outside because of their parents' fears. After blogging about this, I received an email from Enrique, a parent in Austin. He explained:

"Bottom line is that we live in a society of fear; it is unfortunate but true. As a parent, I will admit that I protect my daughter immensely, and I don't let my daughter go out to areas I can't see her. Much different when I was a kid. Am I being over protective? Maybe. But it is the way it is. Is it depressing? No it is not as we keep her busy very busy w/o making it depressing :-)"

Rather than simply enacting physical restrictions, En rique and many other parents chose to limit their children's mobility by instituting extensive schedules and structure. Research showing an inverse correlation between activities and deviance (Mahoney et al. 2005) furthered parents' views that instituting strong structures was beneficial to teens.

I interviewed teens whose social worlds were limited through such structuring and found that many were burned out. My interview with Myra, a white 15-year-old from Iowa, turned tragically comedic when "lack of time" became a refrain as she explained why she did not do various things. From Czech to track to orchestra to 
work in a nursery, her entire schedule had been organized by her mother. Myra did not like all of these activities, but she was resigned to keeping to her mother's schedule because her mother thought it was important. When I asked her if there were anything she did that her mother did not choose, she laughed and told me Spanish Club. Lack of freedom and control over her schedule was a sore topic for Myra. At one point, she noted with an exasperated tone that weekends were no freer than weekdays:

"Usually my mom will have things scheduled for me to do. So I really don't have much choice in what I'm doing Friday nights ... I haven't had a free weekend in so long. I cannot even remember the last time I got to choose what I wanted to do over the weekend."

While Myra's mother likely meant well, Myra was exhausted and felt socially disconnected because she did not have time to connect with friends outside of classes. Many teens who cite time as a limiting factor reference it in combination with parental restrictions. Nicholas, a white 16-year-old from Kansas, told me that he much preferred hanging out with friends in person than online, but that his parents made this impossible. He continued on to note, "I guess not enough free time, too 'cause with me, it's sports. I can't go out and do anything after school."

Physical mobility is also curtailed through structural forces, such as the limited public spaces that welcome teens and lack of transportation options. Many food, shopping, and entertainment venues limit teens explicitly or implicitly and some 
venues have installed a new sound technology to ward off teens through a highpitched sound that only they can hear (Lyall 2005). Even when there are places to go, teens often struggle to get there. In suburban Texas, white 15-year-old Aaron says that he and his friends do not go out "because we don't have cars." Getting a car is often a pivotal moment in teens' lives. As Tara, the 16-year-old from Michigan, explained, "The car is definitely freedom."

Just as adults restrict teens' mobility and freedom to access public spaces, so too do they curtail teen access to online spaces. As discussed above, the moral panic around MySpace prompted many parents to respond by forbidding or limiting access. Additionally, schools use filters to ban access and websites themselves try to ban anyone under 13 from joining the site. While these restrictive efforts parallel those parents take to limit access to public spaces, they are also different because of how and why networked publics are accessed.

While it is possible to curtail access to physical public spaces by keeping teens at home, the Internet allows many teens to access networked publics without leaving home. ${ }^{12}$ Many parents believe that the Internet has tremendous value and few are interested in cutting off all access to the Internet. Yet when teens have Internet access—either at home or school—parents have less control over which networked publics teens access. It is easier for motivated teens to work around filters or avoid

\footnotetext{
${ }^{12}$ Interestingly, in Ghana, where Internet access requires visiting cafés, elders condemn these cafés because it means children are not at home, school, or church. The fears there are less about safety than discipline (Burrell 2007: 70-106).
} 
verbal restrictions than it is for them to sneak out of the house. Many parents are aware of this and, as a result, those who want to control their children's online encounters tend to focus more on limiting their privacy than their mobility.

\subsubsection{Limiting Privacy}

Limiting teenagers' privacy is another mechanism of control. Parents often told me that they thought it was important and their responsibility to know everything happening in their children's lives in order to be good parents, even if that meant invading their privacy, and teens frequently complained about the lack of privacy. What I heard from parents is echoed in online parenting forums that note that "privacy is a privilege" and "one freedom earned by a child is privacy" (Various 2008). The latter poster also noted that rules should come from a place of "protection and love, not the need to be controlling" (Various 2008).

How this plays out varies by household. At one extreme are "helicopter parents" who actively monitor every aspect of their children's lives and intervene on their children's behalf. More commonly, parents simply try to "overhear" everything that happens at home. In Michigan, white 16-year-old Bianca told me that she has no privacy with her family. She noted that she could not have a conversation with her best friend because "my family butts in to everything." She complains that her parents listen in on her conversations and even interrupt for clarifications. Her best friend, white 16-year-old Sasha, concurs, noting that her mother is also extremely nosy. 
Many of the teens I interviewed did not see home as a private space because they lacked control there. Cachi, a Puerto Rican 18-year-old in Iowa, felt that home could not be private because "there's always somebody there." Elsewhere in Iowa, white 15year-old Scott shared similar thoughts, noting that home could be private only when he was alone. He pointed to the fact that he was not allowed locks on his doors as a sign of his limited access to privacy. Even when teens had their own bedrooms with locked doors, they did not always feel confident that what was said there would not be overheard. Sam, the 17-year-old from Iowa, explained that he could not talk on the phone in his bedroom because "you never know what's going to go through those walls." Other teens complain that their parents listen in on their phone calls; this is one reason why teens value mobile phones.

While issues of privacy predate the Internet, the Internet raises new questions about privacy. In one online parenting community, a New York mother extends the privilege argument to the Internet. "I do not believe teenagers 'need' privacy-not when it comes to the Internet. I track everything my kids do online. I search their bedrooms too. I'm the parent-I'm not their friend" (MissyChrissy 2007). In a different thread (MissyChrissy 2008), she responds to a teen who is annoyed when parents look over teens' shoulders by stating,

"Annoying or not, I do it and will always do it. It's MY computer. I also log in and check their history, and track where they go, who they talk to ... everything. I'm a mom. It is my responsibility to protect them. I wouldn't let them talk to strangers 
'irl'13 so why would I let them do it online without supervising? That's just foolish, imo ${ }^{14}$. If my girls don't like my spying, they're free to not use the computer."

Teens view this issue differently. Kat, a white 15-year-old in Massachusetts, willingly gave her mother her Facebook profile, but she finds her mother's hovering annoying. "When I'm talking to somebody online, I don't like when they stand over my shoulder, and I'll be like, 'Mom, can you not read over my shoulder?' Not that I'm saying something bad. It just feels weird. I don't like it." Many teens, like Kat, are more discomforted by the invasive surveillance than the loss of privacy. Such panoptic approaches make them feel as if they have done something wrong, even when they know they have not.

While some parents believe that teens have no right to privacy whatsoever, a more common view is that because the Internet is "public," parents have the right to look at anything that teens post there. This view often upsets teens who see privacy from a different angle. In a Youth Radio episode, white 17-year-old Bly from Maine argues that just because something is publicly accessible does not mean that everyone should have the right to look.

"My mom always uses the excuse about the Internet being "public" when she defends herself. It's not like I do anything to be ashamed of, but a girl needs her

\footnotetext{
13 "irl" means "in real life"

14 "imo" means "in my opinion"
} 
privacy. I do online journals so I can communicate with my friends, not so my mother could catch up on the latest gossip of my life."

Bly believes that her mother should recognize her privacy and respect it by not looking at things that are not intended for her. Bly's mother sees her journal as a publication available for the general public and, as she notes, "I feel I'm part of the general public, so I can view it." They are at odds because they view "public-ness" and privacy differently. For Bly, privacy is not a technical feature, but a social contract. For Bly's mother, anything that is accessible to anyone is fundamentally not private.

The Internet has complicated how teens manage issues of privacy, particularly in the context of adult-teen relations. Even when teens use privacy settings to limit access, they are often incapable of keeping content from those who know them and who are determined to gain access. While many teens block their parents on social network sites, I heard multiple accounts of how parents gained access to teen profiles because the teens forgot to log out of their accounts. This scenario parallels that of parents who read their children's diaries because they left them unlocked in their bedrooms. Because of snooping parents, some teens think obscurity is a better privacy tool for preventing parents' access than technical barriers.

Teens' privacy was limited long before the Internet, but the persistent nature of the Internet makes it easier for parents to track everything that teens do and say. Summer, the 15-year-old from Michigan, tells me that it is much more difficult to 
maintain privacy online than in the park because, in the park, "you can see when there's people around you and stuff like that. So you can like quickly change the subject." Knowing the audience and not having to face the complications presented by the properties of networked publics makes it easier to control the situation such that the park "can be very private but it can also be very public at the same time." Online, Summer does not have that advantage and so she is more cautious.

The collapse of public and private makes it harder for teens to control parents' access and, thus, secure their privacy. Additionally, because many parents believe that they have a right to know what their children are saying online, new technologies have emerged that allow parents to track every keystroke and every mouse click. It is not clear whether or not these parents would attach a spy-cam to their children if they could.

\subsubsection{Network Effects of Control}

For many teens, the value of access to publics and privacy is not simply about personal freedoms, but about their ability to participate in social situations with friends and peers. Mobility is an issue because teens want to be able to access social spaces where other teens gather, both online and off. Privacy is important because teens want to be able to interact with their peers differently from how they interact with their parents. For teens, mobility and privacy are about control. 
While individual parents may make decisions about their child's privacy and mobility, these decisions are configured by broader systems of power and network effects. Parents' decisions are influenced by a general perception of what constitutes good parenting and by the norms in their community. Teens struggle with their parents over these issues, but parents also struggle with each other. Some parents believe that the collective norms are too loose while others see them as too liberal. Parenting with respect to social media is deeply contested and tensions between parents over what is appropriate affect teens as well.

Following up on a news article referencing my work, Mike, a father in Illinois, emailed me to explain that what he perceives to be a decline in societal values has motivated him to be strict with his children.

"The reason my children do not hang out as I used to as a teen is not due to predators necessarily, but due to other teens who have been raised on MTV, lack of parental guidance, and are treated as adults by their parents.... I believe MySpace further sends the entire dynamic down the rabbit hole. If parents took more responsibility for instilling values, morals and standards in their children (versus relying on the educational system, television, and the media), I feel that we could reclaim some of this lost teen freedom for our children.

Many parents like Mike restrict their children from accessing environments in which they may be corrupted, even if that results in a loss of access to social situations. 
Conversely, more permissive parents run into difficulties when they are deemed bad parents and when other parents refuse to let their children socialize together. Vicki, a mother in Pennsylvania, wrote to tell me that she sees her daughter's activities on MySpace as completely normal high-school behavior. "Her friends and she daily talk about who's responding to MySpace email, who's ranked high on their top 10 list, and photos of their latest haircuts or at a concert. It doesn't bother me at all, sounds normal to me." She is aware of what her daughter is doing on MySpace and they talk about how to be safe online. Her biggest concern is that MySpace and IM sometimes distract her daughter from doing her homework, but she also sees this as normal high-school behavior. Yet her decisions to be lax have affected her daughter in a different way.

"One family already banned their daughter from our house and my daughter from their house due to our permissiveness and my daughter's MySpace profile where she indulged in a little profanity and described a summer trip to Ozzfest (I was there, but not mentioned on the web site-big deal.)"

Vicki believes that it is important to maintain an open and trusting relationship with her daughter, but there is general pressure in her community to install software that will restrict what teens can do online. She worries that such an approach to parenting would create an unnecessary "battleground" and destroy the trust that she has worked to build with her daughter. 
When it comes to social media, parenting approaches are highly contested. Just as teens struggle to make sense of norms when socializing on these sites, parents struggle to work out what norms make sense for parenting with respect to these sites. Vicki and Mike approach parenting from entirely different positions, yet neither approach is normative in their communities. This does not affect just their relationships with their children but the ways in which their children can relate to other teens.

\subsection{In the Pursuit of Freedom}

Most teens I interviewed joined social network sites to interact with friends and peers, often to escape structural and social limitations they faced in other contexts. Many believed that MySpace and Facebook were effectively teen space. In a class assignment about the Internet, one teen wrote that teens like the Internet because "it is under our control so we don't have to listen to anyone to tell us what to do or not to do." While teens lack control over many physical spaces where adults dictate norms, the Internet offered uncharted territories for teens to define the norms and social dynamics. At the same time, by trying to control a separate space, teens replicated ongoing age segregation.

In asserting control over these sites, teens sought the freedom to interact on their own terms. Unfortunately, as these sites grew in popularity and adults became aware of teen participation, many were nonplussed that teens had found an outlet 
where adults had limited control over their behavior and a new set of fears broke out, resulting in adults' efforts to reassert control.

Embedded in adults' attempts to limit access and privacy was a reinforcement of an age-segregated, hierarchical society. Yet in many ways, networked technologies destabilize adult control. The technology allows teens to gather when their physical mobility is curtailed and technologically savvy teens can work around most technical measures introduced by adults. While the technology offers new potential for teen agency, many teens reproduce adult fears, accept adult restrictions, and reinforce normative adult values. Still, for some, networked publics remain a valuable outlet for escaping structural limitations.

The most disappointing aspect of adults' efforts to assert control over networked publics is a failure to recognize the potential value of participation in these spaces and other public environments. Networked publics—especially those communities of interest that encourage creativity and action-are often a valuable space for learning (Ito et al. 2008). Likewise, fears over interactions between adults and teens and the reinforcement of age segregation prevent valuable opportunities for learning. Intergenerational interactions are often extremely valuable for teensespecially high-risk teens (Ungar 2004)—because they help teens make sense of the world around them.

Access to public spaces is also a core site of learning. Emily, the white 16-year-old in Pennsylvania, told me that being exposed to different public situations is 
important for learning what is appropriate. She notes, "If you walk in and see what others are doing, then it kind of teaches you what you should do too." Her 14-yearold brother, Anthony, concurs and adds that "our parents teach us how we should behave in public." Both Emily and Anthony believe that whether through modeling or direct lessons, adults and access to public spaces are important for learning about social situations.

While fears have driven and continue to drive the ways in which adults shape teens' lives, teens continue to find ways of meeting their needs despite the restrictions they face. That said, when opportunities are made available for teens to interact with adults in a positive manner or to assert control over their environments, there is a tremendous potential for learning. 


\section{Chapter 7: Lasting Impressions}

In this dissertation, I have mapped out how many American teenagers have adopted social network sites as a core component of their everyday experiences and the ways in which their engagement has reshaped salient aspects of their lives. I have emphasized how technology inflects practices concerning identity, peer socialization, and relationships with adult society. In doing so, I have considered how specific properties of social network sites—namely persistence, searchability, replicability, and scalability-have played a role or a forcing function in prompting shifts in practice. I have also looked at how teens handle many of the significant dynamics that have arisen as a result of networked publics, namely invisible audiences, collapsed contexts, and the blurring of public and private. In this final chapter, I want to highlight that which I believe is especially interesting concerning my findings and their implications. My reflections are organized around three areas of interest: lessons from the everyday lives of teens, the significance of publics, and the future of networked publics.

\subsection{Lessons from the Everyday Lives of Teens}

Nineteenth-century French critic Jean-Baptiste-Alphonse Karr famously quipped, "Plus ça change, plus c'est la même chose. (The more things change, the more they are the same.)" While networked publics have brought about the potential for new configurations of sociality and public life, teens' engagement with social 
network sites reveals a continuation of earlier practices inflected in new ways. Teens are still engaged in complex processes of identity work to locate themselves in social worlds that are predominantly defined by school, religious organizations, family, and geography. Most are still looking to be validated by peers and to gain status in social worlds that predate the Internet. They still struggle to gain acceptance in an adultnarrated society and to have control over their lives as they come of age. At the core, for most teens, not much has changed with respect to the central dynamics of youth and the social frame in which they occur.

That said, the ways in which networked technology has reshaped social conditions have resulted in a wide range of shifts in how these processes unfold. Features like the public articulation of Friends on social network sites have required teens to develop new strategies for managing their social relations and to account for how their digital performance of identity is shaped by their peers' choices in how they in turn display themselves online. This has complicated unequal friendships, prompting a different, more layered form of social drama, which is further complicated by the role that technology plays in teen dramas more broadly. While balancing friendships has always been a part of teen life, relationships have primarily been implicitly_not explicitly_marked. Even if the core practice is not different, this simple technical change creates ripple effects in the social fabric of teens' lives.

Social media is not inherently attractive to teens, but networked publics are frequently valued as spaces to hang out. My findings show that teens are drawn to 
social media collectively and that individuals choose to participate because their friends do. The appeal is not the technology itself-nor any particular technologybut the presence of friends and peers. When asked, teens consistently reported that they would prefer to socialize in physical spaces without constant parental oversight. Given that this is not an option for many of them and that many have more access to networked publics than to unmediated public spaces, social network sites are often an accepted alternative. Those who do actively socialize offline appear to use social network sites differently from those who are restricted. While the latter are deeply engaged in hanging-out activities, the former use these tools to share media, gather information, and communicate between offline encounters. The differences in practice show the ways in which these tools can be used at many different levels.

Teens do not possess a natural capability that enables them to understand how to navigate social media or the resultant dynamics, but they are learning to do so alongside their broader efforts to understand social life. In this way, they are learning to make sense of publics and social worlds that fundamentally include the properties and dynamics of networked technologies. Unlike adults, who are relearning how to behave in public because of networked technologies, teens are simply learning how to behave in public with networked publics in mind.

While teenagers are actively learning as a part of engaging with social media, their participation in public social settings—networked or not-is broadly frowned upon as unsafe or dismissed as frivolous. Although learning to navigate social worlds 
is a critical skill that teens develop through interaction with peers and adults, it is frequently unrecognized in lieu of the skills taught through formal education. One of the most notable shifts I observed in the structural conditions of today's teens, compared to those of earlier decades, involves their limited opportunities for un regulated, unstructured social interaction. Their desire to connect with others is too frequently ignored or disregarded, creating a context in which many must become creative in making space for maintaining connections outside the control of adults.

Unfortunately, teens' efforts to sidestep adults while seeking social spaces of their own have furthered intergenerational conflict fueled by fears and worries. This narrative is not new, but parents are unable to maintain control in this networked context simply by limiting the physical mobility and freedoms of their children. Through the use of technology, teens are able to socialize with others from inside the boundaries of their homes. This presents new freedoms for teens, but it also provokes new fears among adults.

In public discourse, there is a tendency to claim that all has changed or that nothing has, to argue that technology is utterly destroying teens' lives or providing for their salvation. The reality, as evidenced by my findings, is that none of these extremes is accurate. The shifts in teens' lives are often subtle and connected to broader societal trends. Furthermore, as is true of technology more broadly, social media is neither good nor bad, nor is it neutral (Kranzberg 1986). Throughout this 
document are examples of teens' using social media for both productive and destructive purposes. Some teens use these tools to achieve greater freedoms and others find their same social conditions reinforced. What gives hope (and fear) to adult society is the potential of networked publics. The key is for adults, and society more broadly, to engage with these issues and help guide teens in making healthy decisions that allow them to leverage social media in positive ways as part of their everyday lives.

\subsection{The Significance of Publics}

Publics have long been implicated in teenagers' coming-of-age story. Teens push for access to publics because of the ways in which these spaces and audiences help them work through identity and make sense of social life. The desire to be in public predates the Internet, but networked publics offer new possibilities to further these longstanding desires. Publics are woven throughout my dissertation, but their significance is often understated in my analysis.

Identity is fundamentally about locating oneself within a cultural context. As teenagers seek to understand who they are, they are driven to public life, both to consume different ways in which identity is constructed and in their efforts to mark themselves accordingly. Baudelaire's Parisian flâneur enters the public to see and be seen. Teenagers approach publics in a similar vain. Like the flâneur, teens use fashion to convey information about their identities. Physical public spaces are still important to teens with respect to identity, but when their access is curtailed, their 
opportunities for self-expression are limited. Networked publics introduce new opportunities for seeing and being seen, but the processes of self-expression are also shaped by the structural differences of these environments.

Sociality is defined both by interacting with others and by participating in society more broadly. Publics play an important role in sociality by providing a context in which people can connect with others and locate themselves in terms of status. Although school publics serve this purpose for many teens, the limited interaction time at school prompts teens to find new ways to connect with their peers. While friendships can be managed privately, teens seek out places where their peers congregate in order to build new connections and seek intimate relationships. Movie theaters and malls are still desirable spaces for gathering with peers, but when physical mobility is limited, networked publics offer a reasonable alternative. For most teens in my study, networked publics primarily serve as an extension of school publics or provide an opportunity to mix peers from school, religious organizations, camp, and activities. For marginalized and ostracized teens, networked publics can provide alternate options. Yet even teens who fit in at school find value in the possible encounters with friends of friends. The teen years are marked by an interest in building new connections and socializing broadly. When adults try to curtail sociality, teens are often driven to find spaces and contexts in which they can connect with others. 
The processes of identity and sociality are frequently entwined, especially when teens leverage identity displays to seek status and validation and when teens try to build connections with others who share similar identity-based interests. Likewise, the publics in which teens move are layered and interconnected. School publics often extend beyond the school yard and mix with other publics. In such contexts, teens may seek status from school peers through the validation of others in those other publics. These same dynamics are mirrored and magnified online, but they are also shifted because of how sociality and identity are negotiated in networked publics.

Publics are also at the crux of adult fears and teens' desire for agency. While teens seek the unpredictable potential of publics, this is precisely what worries adults who prefer more controlled settings. In many ways, access to publics has become an adult privilege in contemporary society, especially as teenagers are limited in their mobility and as brokers of unmediated spaces discourage teen participation. Because of social factors, few teens are interested in intergenerational publics, but many fervently seek access to peer publics where adult power is not in play. Teens have long struggled to find a place for themselves; they have consistently formed counterpublics within broader structures. Yet when they do, adults typically demonize them, the identity markers they use, and the publics they co-opt. The demonization of MySpace is akin to the demonization of malls and parking lots that took place when I was growing up. 
The struggles that have unfolded over online freedoms parallel those over offline spaces and adults have translated their tactics for regulating teens into digital contexts. While some fears have subsided since my fieldwork, new ones have emerged and teens continue to be vulnerable to the ways in which adults seek to maintain power and authority over them.

The inability to access publics is an explicit reminder of teens' marginalized position within society. Teens' struggle to access these spaces is driven by their desire for agency and status in a world defined by adults. At the same time, adults' efforts to curtail teens' access to publics are driven by their fears and desire for control. When well-intentioned parents limit access to publics out of fear of potential dangers, they fail to provide their children with the tools to transition into adult society. This may have other unexpected consequences, including isolating teens from political life and curbing their civic engagement. I believe that the practice of maximum control and restrictions infantilizes teenagers, making them more dependent on or resentful of adults and adult society.

\subsection{The Future of Networked Publics}

The properties that are fundamental to networked publics and the dynamics that emerge from them are not wholly new. In many cases, they are an extension of the factors that Meyrowitz (1985) identified when speaking of television. What is unique are the ways in which they interrelate and their widespread significance as networked publics became broadly accessible. What I mark in this dissertation is not 
an end state. Publics will continue to be transformed by structural changes resulting from the interplay of new technologies and their adoption.

An additional shift, not analyzed or discussed in this dissertation, is already under way. Mobile phones, the increased availability of wireless frequencies, and new gen res of handheld devices are increasing the ubiquity of computing. Although mobile phones have been popular for some time (Ito 2005), the emergence of new "smart" phones and devices that leverage open wireless networks are introducing new interaction paradigms. With its voice and texting capabilities, the mobile has revolutionized how people communicate, but those interactions are still primarily one-to-one. Newer phones and emergent practices are creating a technical and social context for the rise of mobile networked publics.

Most of the teens I interviewed owned a mobile phone, but many craved the newer devices that supported Internet access and different genres of social media. Those who owned more advanced devices, like the Sidekick, revealed how newer technologies supported different interactive practices. As Shean, a black 17-year-old from Los Angeles, explained,

"It's like a mini-computer, you can just take it with you. If you're lost, you can get on MapQuest on your Sidekick. When you have your Sidekick, you can text message so fast-and it has AIM. And everybody has AIM. And all my friends have Sidekicks so it's like the new network for kids." 
This example highlights one way in which adoption of advanced mobile technology alters behavior.

Mobile technologies introduce a new property for consideration(dis)locatability. (Dis)locatability points to the ways in which mobile networked interactions are simultaneously independent of and deeply connected to physical location. Through the mobile, teens are able to interact with one another regardless of where they are; there is no need to be physically tethered to a specific place to connect or to be present at a known location to be reached. While dislocatability is already widely felt, global positioning system (GPS) technologies are reintroducing location into the experience. Users of newer phones can grab information related to their location, project their location publicly, and use their devices to find others who are nearby. This is the property of locatability. Together, dislocatability and locatability introduce new possibilities for how physicality and spatiality will intersect with networked publics.

It is too early to examine the role newer mobile phones will play in shaping practice and public life, but it is likely that the properties and dynamics of publics will be once again be transformed as networked publics go mobile. It is also unlikely that this will be the last innovation that will reconfigure the infrastructure of public life. New technologies will continue to emerge and the ways in which they are adopted and integrated into everyday life will reshape practice. There is no linear progression to development and other forces may dampen technological and 
cultural innovation (Zittrain 2008), but the structural conditions of public life will likely evolve.

My dissertation highlights how teenagers have adapted to and co-opted one technological shift, but new ones will emerge and require examination. Publics will continue to evolve and bring with them new dynamics that shift practices. By looking to the core properties and dynamics of networked publics, my analysis reveals one way to examine the structural transformations of public life that are likely to occur as new technologies emerge. Furthermore, the intergenerational struggles over networked publics also suggest that adoption, adaptation, and cooption of new shifts are unlikely to be experienced simultaneously.

Teenagers' experiences shed light on the shifts brought on by the transformation of publics because of their lack of familiarity with and indoctrination into broadly understood publics controlled by adults. They accept—and learn to make sense ofthe publics to which they have access, while adults often stumble because they are forced to develop new skills and let go of old presuppositions. In learning how to make sense of publics that are different from those with which their parents are comfortable, teenagers reveal valuable techniques for interpreting and reworking publics. Their experiences provide valuable insight for understanding how publics are transformed by structural forces. 


\section{Bibliography}

Abbott, Chris. 1998. "Making Connections: Young People and the Internet.” Pp. 84-105 in Digital Diversions: Youth Culture in the Age of Multimedia, edited by Julian Sefton-Green. London, UK: UCL Press.

Adamic, Lada A. and Natalie Glance. 2005. “The Political Blogosphere and the 2004 U.S. Election: Divided They Blog." Proceedings of Knowledge Discovery in Data, Chicago, Illinois: ACM, 36-43.

Adams, Kenneth. 2003. "The Effectiveness of Juvenile Curfews at Crime Prevention." The ANNALS of the American Academy of Political and Social Science 587(1): 136-159.

Anderson, Benedict. 2006a. Imagined Communities: Reflections on the Origin and Spread of Nationalism. New ed. New York: Verso.

Anderson, Chris. 2006b. The Long Tail: Why the Future of Business Is Selling Less of More. New York: Hyperion.

Appadurai, Arjun. 1996. Modernity at Large: Cultural Dimensions of Globalization. Minneapolis, MN: University of Minnesota Press.

Arendt, Hannah. 1998. The Human Condition. Chicago, IL: University of Chicago Press.

Aries, Philippe. 1962. Centuries of Childhood: A Social History of Family Life. New York: Random House.

Arnold, Rebecca. 2001. Fashion, Desire and Anxiety: Image and Morality in the 20th Century. Rutgers, NJ: Rutgers University Press. 
$\mathrm{Au}$, Wagner James. 2008. The Making of Second Life: Notes from the New World. New York: Collins.

Auslander, Philip. 1999. Liveness: Performance in a Mediatized Culture. London, UK: Routledge.

Bahney, Anna. 2006. “Don't Talk to Invisible Strangers.” The New York Times, March 9. Retrieved December 3, 2008 (http://www.nytimes.com/2006/03/09/ fashion/thursdaystyles/09parents.html).

Balsamo, Anne. 1995. "Signal to Noise: On the Meaning of Cyberpunk Subculture." Pp. 347-368 in Communication in the Age of Virtual Reality, edited by Frank Biocca and Mark R. Levy. Mahwah, NJ: Lawrence Erlbaum.

Barlow, John Perry. 1996. “A Declaration of Independence of Cyberspace.” Retrieved December 3, 2008 (http://homes.eff.org/ barlow/DeclarationFinal.html).

Baron, Naomi S. 2008. Always On: Language in an Online and Mobile World. Oxford, UK: Oxford University Press.

Battelle, John. 2005. The Search: How Google and Its Rivals Rewrote the Rules of Business and Transformed Our Culture. New York: Penguin.

Bauerlein, Mark. 2008. The Dumbest Generation: How the Digital Age Stupefies Young Americans and Jeopardizes Our Future (Or, Don't Trust Anyone under 30). New York: Tarcher.

Bausch, Suzy. 2007. Teens Who Visit Both MySpace and Facebook Drive Time Spent at the Social Networking Sites. Nielsen/NetRatings. Retrieved December 3, 2008 (http://www.nielsen-netratings.com/pr/pr_070920.pdf). 
Baym, Nancy K. 1993. "Interpreting Soap Operas and Creating Community: Inside a Computer-Mediated Fan Culture.” Journal of Folklore Research 30(2/3): 143177.

Benjamin, Walter. 1969. “The Work of Art in the Age of Mechanical Reproduction.” Pp. 217-252 in Illuminations, edited by Walter Benjamin. Translated by Harry Zohn. New York: Schocken Books.

Benkler, Yochai. 2006. The Wealth of Networks: How Social Production Transforms Markets and Freedom. New Haven, CT: Yale University Press.

Bennett, Andy. 2004. "Virtual Subculture? Youth, Identity and the Internet." Pp. 162-172 in After Subculture, edited by Andy Bennett and Keith Kahn-Harris. New York: Palgrave Macmillan.

Berman, Joshua and Amy S. Bruckman. 2001. “The Turing Game: Exploring Identity in an Online Environment." Convergence 7(3): 83.

Berndt, Thomas J. 1996. "Exploring the Effects of Friendship Quality on Social Development." Pp. 346-365 in The Company They Keep: Friendship in Childhood and Adolescence, edited by William M. Bukowski, Andrew F. Newcomb, and Willard W. Hartup. Cambridge, UK: Cambridge University Press.

Bettie, Julie. 2003. Women without Class: Girls, Race, and Identity. Berkeley: University of California Press.

Bijker, Wiebe E., Thomas Parke Hughes, and Trevor J. Pinch, eds. 1987. The Social Construction of Technological Systems: New Directions in the Sociology and History of Technology. Cambridge, MA: MIT Press.

Boellstorff, Tom. 2008. Coming of Age in Second Life: An Anthropologist Explores the Virtually Human. Princeton, NJ: Princeton University Press. 
Bourdieu, Pierre. 1984. Distinction: A Social Critique of the Judgement of Taste. London, UK: Routledge.

boyd, danah. 2002. "Faceted Id/entity: Managing Representation in a Digital World." Master's Thesis, Media Arts and Sciences, Massachusetts Institute of Technology, Cambridge, MA.

boyd, danah. 2006a. "Friendster Lost Steam. Is MySpace Just a Fad?” Apophenia, March 21, 2006. Retrieved December 3, 2008 (http://www.danah.org/papers/FriendsterMySpaceEssay.html).

boyd, danah. 2006b. "Friends, Friendsters, and MySpace Top 8: Writing Community into Being on Social Network Sites." First Monday 11(12). Retrieved June 26, 2008 (http://www.firstmonday.org/issues/issue11_12/boyd).

boyd, danah. 2007. "Why Youth (Heart) Social Network Sites: The Role of Networked Publics in Teenage Social Life." Pp. 119-142 in MacArthur Series on Digital Learning-Youth, Identity, and Digital Media Volume, edited by David Buckingham. Cambridge, MA: MIT Press.

boyd, danah. 2008a. "Facebook’s Privacy Trainwreck: Exposure, Invasion, and Social Convergence." Convergence 14(1): 13-20.

boyd, danah. 2008b. "None of This Is Real." Pp. 132-157 in Structures of Participation in Digital Culture, edited by Joe Karaganis. New York: Social Science Research Council.

boyd, danah m. and Nicole B. Ellison. 2007. "Social Network Sites: Definition, History, and Scholarship." Journal of Computer-Mediated Communication 13(1): article 11. Retrieved December 3, 2008 (http://jcmc.indiana.edu/vol13/issue1/boyd.ellison.html). 
Bradley, Michael J. 2003. Yes, Your Teen is Crazy!: Loving Your Kid Without Losing Your Mind. Gig Harbor, WA: Harbor Press, Inc.

Brake, David. 2008. "Shaping the 'me' in MySpace: The Framing of Profiles on a Social Network Site.” Pp. 285-300 in Digital Storytelling, Mediatized Stories: Self-representations in New Media, edited by Knut Lundby. New York: Peter Lang.

Brin, David. 1999. The Transparent Society: Will Technology Force Us to Choose between Privacy and Freedom? New York: Basic Books.

Bruckman, Amy and Mitch Resnick. 1995. “The MediaMOO Project:

Constructionism and Professional Community." Convergence 1(1): 94-109.

Bryant, J. Alison, Ashley Sanders-Jackson, and Amber M.K. Smallwood. 2006.

“IMing, Text Messaging, and Adolescent Social Networks." Journal of Computer-Mediated Communication 11(2): article 10. Retrieved December 3, 2008 (http://jcmc.indiana.edu/vol11/issue2/bryant.html).

Buchanan, Elizabeth A. 2004. Readings in Virtual Research Ethics: Issues and Controversies. Hershey, PA: Information Science.

Buckingham, David. 2000. After the Death of Childhood: Growing Up in the Age of Electronic Media. Cambridge, UK: Polity Press.

Buckingham, David. 2007. "Introducing Identity." Pp. 1-24 in MacArthur Series on Digital Learning-Youth, Identity, and Digital Media Volume, edited by David Buckingham. New York: MIT Press.

Burrell, Jenna. 2007. "Producing the Internet and Development: An Ethnography of Internet Café Use in Accra, Ghana." PhD Thesis, Sociology, The London School of Economics and Political Science, London, UK. 
Burrell, Jenna. Forthcoming. "The Fieldsite as a Network: A Strategy for Locating Ethnographic Research." Field Methods.

Calhoun, Craig. 1992a. Habermas and the Public Sphere. Cambridge, MA: The MIT Press.

Calhoun, Craig. 1992b. "Introduction." Pp. 1-50 in Habermas and the Public Sphere, edited by Craig J. Calhoun. Cambridge, MA: The MIT Press.

Castells, Manuel. 1996. The Rise of the Network Society. Cambridge, MA: Blackwell.

Castells, Manuel. 2004. The Power of Identity. Vol. 2. Malden, MA: Blackwell.

Chen, Wenhong and Barry Wellman. 2005. "Minding the Cyber-Gap: The Internet and Social Inequality." Pp. 523-545 in The Blackwell Companion to Social Inequalities, edited by Mary Romero and Eric Margolis.

Cherny, Lynn. 1999. Conversation and Community: Chat in a Virtual World. Stanford, CA: CSLI Publications.

Chicago v. Morales, 527 U.S. 41 (1999).

Chudacoff, Howard. 2007. Children at Play: An American History. New York: NYU Press.

Chudacoff, Howard P. 1989. How Old Are You?: Age Consciousness in American Culture. Princeton, NJ: Princeton University Press.

Clifford, James. 1997. Routes: Travel and Translation in the Late Twentieth Century. Cambridge, MA: Harvard University Press.

Clifford, James and George E. Marcus, eds. 1986. Writing Culture: The Poetics and Politics of Ethnography. Berkeley, CA: University of California Press. 
Clippinger, John. 2007. A Crowd of One: The Future of Individual Identity. New York: Perseus.

Cochran, J. Thomas. 1997. A Status Report on Youth Curfews in America's Cities. United States Conference of Mayors. Retrieved December 3, 2008 (http://www.usmayors.org/publications/curfew.htm).

Cohen, Rachel. 2003. "Livewire: Web Sites Try to Make Internet Dating Less Creepy.” Reuters, July 5. Retrieved July 5, 2003 (http://asia.reuters.com/ newsArticle.jhtml? type=internetNews\&storyID=3041934).

Cohen, Stanley. 1972. Folk Devils and Moral Panics: The Creation of the Mods and Rockers. London, UK: MacGibbon and Kee.

Corsaro, William A. 1985. Friendship and Peer Culture in the Early Years. Norwood, NJ: Ablex.

Corsaro, William A. 1997. The Sociology of Childhood. Thousand Oaks, CA: Pine Forge Press.

Cotterell, John. 1996. Social Networks and Social Influences in Adolescence. London, UK: Routledge.

Crane, Diana. 2000. Fashion and Its Social Agendas: Class, Gender, and Identity in Clothing. Chicago, IL: University of Chicago Press.

Crossley, Nick and John Michael Roberts. 2004. After Habermas: New Perspectives on the Public Sphere. Oxford, UK: Blackwell.

Csikszentmihalyi, Mihaly and Reed Larson. 1986. Being Adolescent: Conflict and Growth in the Teenage Years. New York: Basic Books.

Davis, Fred. 1992. Fashion, Culture, and Identity. Chicago, IL: University of Chicago Press. 
de Certeau, Michel. 2002. The Practice of Everyday Life. Berkeley and Los Angeles, CA: University of California Press.

Donath, Judith S. 1999. "Identity and Deception in the Virtual Community." Pp. 29-59 in Communities in Cyberspace, edited by Peter Kollock and Marc Smith. London, UK: Routledge.

Donath, Judith. 2007. "Signals in Social Supernets." Journal of Computer-Mediated Communication 13(1): article 12. Retrieved December 3, 2008 http://jcmc.indiana.edu/vol13/issue1/donath.html).

Donath, Judith and danah boyd. 2004. "Public Displays of Connection.” BT Technology Journal 22(4): 71-82.

Duncombe, Stephen. 2008. Notes from Underground: Zines and the Politics of Alternative Culture. Bloomington, IN: Microcosm.

Eckert, Penelope. 1989. Jocks and Burnouts: Social Categories and Identity in High School. New York: Teachers College Press.

Eckert, Penelope. 1996. "Vowels and Nail Polish: The Emergence of Linguistic Style in the Preadolescent Heterosexual Marketplace." Pp. 183-190 in Gender and Belief Systems, edited by Natasha Warner, Jocelyn Ahlers, Leela Bilmes, Monical Olver, Suzanna Wertheim, and Meilinda Chen. Berkeley, CA: Berkeley Women and Language Group University of California.

Eder, Donna, Catherine Colleen Evans, and Stephen Parker. 1995. School Talk: Gender and Adolescent Culture. New Brunswick, NJ: Rutgers University Press.

Eisenstein, Elizabeth L. 1980. The Printing Press as an Agent of Change. Vols. 1 and 2 in One. Cambridge, UK: Cambridge University Press. 
Ellison, Nicole, Rebecca Heino, and Jennifer Gibbs. 2006. "Managing Impressions Online: Self-Presentation Processes in the Online Dating Environment." Journal of Computer-Mediated Communication 11(2): article 2. Retrieved December 3, 2008 (http://jcmc.indiana.edu/vol11/issue2/ellison.html).

Ellison, Nicole B., Charles Steinfield, and Cliff Lampe. 2007. "The Benefits of Facebook "Friends:" Social Capital and College Students' Use of Online Social Network Sites." Journal of Computer-Mediated Communication 12(4): article 1. Retrieved December 3, 2008 (http://jcmc.indiana.edu/vol12/issue4/ellison.html).

Elm, Malin Sveningsson. 2008. "How Do Various Notions of Privacy Influence Decisions in Qualitative Internet Research?” Pp. 69-87 in Internet Inquiry: Conversations about Method, edited by Annette N. Markham and Nancy K. Baym. Los Angeles, CA: Sage.

Erikson, Erik H. 1959. Identity and the Life Cycle. New York: International Universities Press.

Fineman, Martha. 1994. The Public Nature of Private Violence: Women and the Discovery of Abuse. New York: Routledge.

Finkelhor, David, Kimberly Mitchell, and Janis Wolak. 2000. Online Victimization: A Report on the Nation's Youth. National Center for Missing and Exploited Children. Retrieved December 3, 2008 (http://eric.ed.gov/ERICWebPortal/recordDetail?accno=ED442039).

U.S. Congress. House of Representatives. Deleting Online Predators Act of 2006. H.R. 5319. 109th Congress, 2006.

Foucault, Michel. 1990. The History of Sexuality, Vol. 1: An Introduction. New York: Vintage. 
Frank, Thomas. 1997. The Conquest of Cool: Business Culture, Counterculture, and the Rise of Hip Consumerism. Chicago, IL: University of Chicago Press.

Fraser, Nancy. 1992. "Rethinking the Public Sphere: A Contribution to the Critique of Actually Existing Democracy." Pp. 109-142 in Habermas and the Public Sphere, edited by Craig Calhoun. Cambridge, MA: The MIT Press.

Gaines, Donna. 1998. Teenage Wasteland: Suburbia's Dead End Kids. Chicago, IL: University of Chicago Press.

Garfinkel, Simson. 2001. Database Nation: The Death of Privacy in the 21st Century. Sebastopol, CA: O’Reilly Media.

Gay, Paul du, Jessica Evans, and Peter Redman. 2001. Identity: A Reader. London, UK: Sage and The Open University.

Geertz, Clifford. 1973. The Interpretation of Cultures. New York: Basic Books.

Gelder, Ken and Sarah Thornton, eds. 1997. The Subcultures Reader. London, UK: Routledge.

Giddens, Anthony. 1991. Modernity and Self-Identity: Self and Society in the Late Modern Age. Palo Alto, CA: Stanford University Press.

Gilbert, James Burkhart. 1986. A Cycle of Outrage: America's Reaction to the Juvenile Delinquent in the 1950s. New York: Oxford University Press.

Gillmor, Dan. 2004. We the Media: Grassroots Journalism by the People, for the People. Sebastopol, CA: O’Reilly Media.

Glassner, Barry. 1999. The Culture of Fear: Why Americans Are Afraid of the Wrong Things. New York: Basic Books. 
Goffman, Erving. 1959. The Presentation of Self in Everyday Life. Garden City, New York: Doubleday.

Goffman, Erving. 1963. Stigma: Notes on the Management of Spoiled Identity. Englewood Cliffs, NJ: Prentice-Hall.

Goffman, Erving. 1966. Behavior in Public Places: Notes on the Social Organization of Gatherings. New York: Free Press.

Goffman, Erving. 1967. Interaction Ritual: Essays on Face-to-Face Behavior. Garden City, NY: Anchor Books.

Goode, Erich and Nachman Ben-Yehuda. 1994. Moral Panics: The Social Construction of Deviance. Oxford, UK: Blackwell.

Gordon, Jane. 2006. “MySpace Draws a Questionable Crowd.” The New York Times, February 26. Retrieved December 3, 2008 (http://www.nytimes.com/ 2006/02/26/nyregion/nyregionspecial2/26ctspace.html).

Gray, Mary L. Forthcoming. Out in the Country: Youth, Media, and the Queering of Rural America.

Green, Nicola. 1999. "Disrupting the Field: Virtual Reality Technologies and 'Multisited' Ethnographic Methods.” American Behavioral Scientist 43(3): 409-421.

Grimmelmann, James. 2004. “Regulation by Software.” Yale Law Journal 114: $1719-1758$.

Grimmelmann, James. 2008. "Facebook and the Social Dynamics of Privacy." The Selected Works of James Grimmelmann. Retrieved December 3, 2008 (http://works.bepress.com/james_grimmelmann/20). 
Grint, Keith and Steve Woolgar. 1997. "Configuring the User: Inventing New Technologies." Pp. 65-94 in The Machine at Work: Technology, Work, and Organization, edited by Keith Grint and Steve Woolgar. Cambridge, UK: Polity Press.

Grinter, Rebecca E. and Margery A. Eldridge. 2001. “y do tngrs luv 2 txt msg?” Pp. 219-238 in Proceedings of Seventh European Conference on ComputerSupported Cooperative Work ECSCW'01, Bonn, Germany: Kluwer Academic Publishers.

Grinter, Rebecca E., Leysia Palen, and Margery Eldridge. 2006. "Chatting with Teenagers: Considering the Place of Chat Technologies in Teen Life." ACM Transactions on Computer Human Interaction 13(4): 423-447.

Habermas, Jèrgen. 1991. The Structural Transformation of the Public Sphere: An Inquiry into a Category of Bourgeois Society. Cambridge, MA: MIT Press.

Hall, G. Stanley. 1908. Adolescence. London, UK: Appleton and Co.

Hall, Stuart and Tony Jefferson. 1976. Resistance through Rituals: Youth Subcultures in Post-War Britain. New York: Routledge.

Hancock, Jeffrey T., Catalina Toma, and Nicole Ellison. 2007. “The Truth about Lying in Online Dating Profiles.” Pp. 449-452 in Proceedings of the SIGCHI conference on Human Factors in Computing Systems. New York: ACM.

Haraway, Donna Jeanne. 1991a. "A Cyborg Manifesto: Science, Technology, and Socialist-Feminism in the Late Twentieth Century.” Pp. 149-181 in Simians, Cyborgs, and Women: The Reinvention of Nature, edited by Donna Jeanne Haraway. New York: Routledge.

Haraway, Donna Jeanne. 1991b. Simians, Cyborgs, and Women: The Reinvention of Nature. New York: Routledge. 
Hargittai, Eszter. 2002. “Second-Level Digital Divide: Differences in People's Online Skills.” First Monday 7(4): 1-20.

Hargittai, Eszter. 2007. "Whose Space? Differences among Users and Non-Users of Social Network Sites." Journal of Computer-Mediated Communication 13(1): article 14. Retrieved December 3, 2008 (http://jcmc.indiana.edu/vol13/issue1/hargittai.html).

Hays, K. Michael. 2000. Architecture Theory since 1968. Cambridge, MA: The MIT Press.

Haythornthwaite, Caroline and Barry Wellman. 2002. “The Internet in Everyday Life: An introduction.” Pp. 3-41 in The Internet in Everyday Life, edited by Barry Wellman and Caroline Haythorthwaite. London, UK: Blackwell.

Hebdige, Dick. 1979. Subculture: The Meaning of Style. London, UK: Routledge.

Hempel, Jessi. 2005. "Protecting Your Kids from Cyber-Predators.” BusinessWeek, December 12. Retrieved December 3, 2008 (http://www.businessweek.com/magazine/content/05_50/b3963015.htm).

Hinduja, Sameer and Justin Patchin. 2008. "Cyberbullying: An Exploratory Analysis of Factors Related to Offending and Victimization." Deviant Behavior 29(2): 129-156.

Hine, Christine. 1998. Virtual Ethnography. London, UK: Sage.

Hine, Thomas. 1999. The Rise and Fall of the American Teenager. New York: Perennial.

Hirsch, E. D., Joseph F. Kett, and James S. Trefil. 2002. The New Dictionary of Cultural Literacy. Boston, MA: Houghton Mifflin.

Hodkinson, Paul. 2002. Goth: Identity, Style and Subculture. Oxford, UK: Berg. 
Hodkinson, Paul and Sian Lincoln. 2008. "Online Journals as Virtual Bedrooms?: Young People, Identity and Personal Space.” Young 16(1): 27-46.

Holloway, Sarah L. and Gill Valentine. 2003. Cyberkids: Children in the Information Age. London, UK: Routledge.

Horne, Christine, Mary Kris Mcilwaine, and Kristie A. Taylor. 2005. "Civility and Order: Adult Social Control of Children in Public Places.” Pp. 181-200 in Together Alone: Personal Relationships in Public Places, edited by Calvin Morrill, David A. Snow, and Cindy White. Berkeley, CA: University of California Press.

Howes, Carollee. 1996. “The Earliest Friendships.” Pp. 66-86 in The Company They Keep: Friendship in Childhood and Adolescence, edited by William M. Bukowski, Andrew F. Newcomb, and Willard W. Hartup. Cambridge, UK: Cambridge University Press.

Ito, Mizuko. 2002. “Engineering Play: Children's Software and the Productions of Everyday Life.” PhD Thesis, Anthropology, Stanford University, Palo Alto, CA.

Ito, Mizuko. 2005. “Introduction: Personal, Portable, Pedestrian.” Pp. 1-18 in Personal, Portable, Pedestrian: Mobile Phones in Japanese Life, edited by Mizuko Ito, Daisuke Okabe, and Misa Matsuda. Cambridge, MA: MIT Press.

Ito, Mizuko. 2008. "Introduction." Pp. 1-14 in Networked Publics, edited by Kazys Varnelis. Cambridge, MA: MIT Press.

Ito, Mizuko, Sonja Baumer, Matteo Bittanti, danah boyd, Rachel Cody, Becky HerrStephenson, Heather A. Horst, Patricia G. Lange, Dilan Mahendran, Katynka Martinez, C.J. Pascoe, Dan Perkel, Laura Robinson, Christo Sims, and Lisa Tripp. 2008. Hanging Out, Messing Around and Geeking Out: Living and 
Learning with New Media. Chicago, IL: The John D. and Catherine T. MacArthur Foundation.

Jagodzinski, Cecile M. 1999. Privacy and Print: Reading and Writing in SeventeenthCentury England. Charlottesville, VA: University of Virginia Press.

James, Allison, Chris Jenks, and Alan Prout. 1988. Theorizing Childhood. Cambridge, UK: Polity.

Jenkins, Henry. 1992. Textual Poachers: Television Fans and Participatory Culture. New York: Routledge.

Jenkins, Henry. 2006. Convergence Culture: Where Old and New Media Collide. New York: New York University Press.

Jenkins, Henry, Katie Clinton, Ravi Purushotma, Alice J. Robinson, and Margaret Weigel. 2006. Confronting the Challenges of Participatory Culture: Media Education for the 21st Century. Chicago, IL: The John D. and Catherine T. MacArthur Foundation. Retrieved December 3, 2008 (http://www.newmedialiteracies.org/files/working/NMLWhitePaper.pdf).

Jerigan, Joy. 2008. "When You Don't Want to Be Facebook Friends." MSNBC, November 18. Retrieved December 3, 2008 (http://www.msnbc.msn.com/id/27775484/).

Johnston, Lloyd D., Patrick M. O’Malley, Jerald G. Bachman, and John E. Schulenberg. 2007. Monitoring the Future: National Results on Adolescent Drug Use. National Institute on Drug Abuse. Retrieved December 3, 2008 (http://monitoringthefuture.org/pubs/monographs/overview2007.pdf).

Jordon, Tim. 2008. Hacking: Digital Media and Technological Determinism. Cambridge, UK: Polity. 
Katz, James and Philip Aspden. 1997. "Motivations for and Barriers to Internet Usage: Results of a National Public Opinion Survey." Internet Research 7(3): $170-188$.

Kaufman, Gil. 2006. “Twenty Students Suspended in Latest Round of MySpaceRelated Busts.” MTV.com, March 3. Retrieved December 3, 2008 (http://www.mtv.com/news/articles/1525313/20060303/index.jhtml).

Kearney, Mary Celeste. 2006. Girls Make Media. New York: Routledge.

Keen, Andrew. 2007. The Cult of the Amateur: How Today's Internet Is Killing Our Culture and Assaulting Our Economy. New York: Doubleday.

Kelty, Christopher M. 2008. Two Bits: The Cultural Significance of Free Software. Durham, NC: Duke University Press.

Kendall, Lori. 2002. Hanging Out in the Virtual Pub: Masculinities and Relationships Online. Berkeley, CA: University of California Press.

Kinney, David A. 1993. "From Nerds to Normals: The Recovery of Identity among Adolescents from Middle School to High School." Sociology of Education 66(1): 21-40.

KNBC. 2006. "Mothers Think Teens Were Lured Away by MySpace.com Suitors.” NBC4.TV, February 26. Retrieved July 8, 2008 (http://www.knbc.com/news/7473070/detail.html).

Knobel, Michele and Colin Lankshear. 2007. "Online Memes, Affinities, and Cultural Production.” Pp. 199-228 in A New Literacies Sampler, edited by Michele Knobel and Colin Lankshear. New York: Peter Lang.

Kornblum, Janet and Mary Beth Marklein. 2006. "What You Say Online Could Haunt You.” USA Today, March 8. Retrieved December 3, 2008 
(http://www.usatoday.com/tech/news/internetprivacy/2006-03-08-facebookmyspace_x.htm).

Kranzberg, Melvin. 1986. “Technology and History: 'Kranzberg's Laws.” Technology and Culture 27(3): 544-560.

Lam, Chuck. 2007a. “Analyzing Facebook Usage by High School Demographic.” Data Strategy, September 14. Retrieved December 3, 2008 (http://datastrategy.wordpress.com/2007/09/14/analyzing-facebook-usage-byhigh-school-demographic/).

Lam, Chuck. 2007b. “Examining MySpace Usage by High School.” Data Strategy, September 19. Retrieved December 3, 2008 (http://datastrategy.wordpress.com/2007/09/19/examining-myspace-usage-byhigh-school/).

Larson, Reed W. 2001. "How US Children and Adolescents Spend Time: What It Does (and Doesn't) Tell Us about Their Development." Current Directions in Psychological Science 10(5): 160-164.

Lassiter, Luke Eric. 2005. "Collaborative Ethnography and Public Anthropology.” Current Anthropology 46(1): 83-106.

Leander, Kevin M. and Kelly K. McKim. 2003. “Tracing the Everyday 'Sitings' of Adolescents on the Internet: A Strategic Adaptation of Ethnography across Online and Offline Spaces.” Education, Communication \& Information 3(2): 211-240.

Leblanc, Lauraine. 1999. Pretty in Punk: Girls' Gender Resistance in a Boys' Subculture. New Brunswick, NJ: Rutgers University Press. 
Lederer, Scott, Jason I. Hong, Anind K. Dey, and James A. Landay. 2004. "Personal Privacy through Understanding and Action: Five Pitfalls for Designers.” Personal and Ubiquitous Computing 8: 440-454.

Lee, Jennifer 8. 2004. "Web Diarists Are Now Official Members of the Convention Press Corps.” The New York Times, July 26. Retrieved December 3, 2008 (http://www.nytimes.com/2004/07/26/politics/campaign/26blog.html).

Lenhart, Amanda, Joseph Kahne, Ellen Middaugh, Alexandra R. Macgill, Chris Evans, and Jessica Vitak. 2008. Teens, Video Games and Civics. Pew Internet and American Life Project. Retrieved December 3, 2008 (http://www.pewinternet.org/PPF/r/263/report_display.asp).

Lenhart, Amanda and Mary Madden. 2007a. Social Networking Websites and Teens: An Overview. Pew Internet and American Life Project. Retrieved December 3, 2008 (http://www.pewinternet.org/PPF/r/198/report_display.asp).

Lenhart, Amanda and Mary Madden. 2007b. Teens, Privacy, and Online Social Networks. Pew Internet and American Life Project. Retrieved December 3, 2008 (http://www.pewinternet.org/PPF/r/211/report_display.asp).

Lenhart, Amanda, Mary Madden, and Paul Hitlin. 2005. Teens and Technology: Youth Are Leading the Transition to a Fully Wired and Mobile Nation. Pew Internet and American Life Project. Retrieved December 3, 2008 (http://www.pewinternet.org/PPF/r/162/report_display.asp).

Lenhart, Amanda, Mary Madden, Alexandra R. Macgill, and Aaron Smith. 2007. Teens and Social Media. Pew Internet and American Life Project. Retrieved December 3, 2008 (http://www.pewinternet.org/PPF/r/230/report_display.asp). 
Lenhart, Amanda, Lee Rainie, and Oliver Lewis. 2001. Teenage Life Online: The Rise of the Instant-Message Generation and the Internet's Impact on Friendships and Family Relationships. Pew Internet and American Life Project. Retrieved December 3, 2008 (http://www.pewinternet.org/PPF/r/36/report_display.asp).

Lessig, Lawrence. 2005. Free Culture: The Nature and Future of Creativity. New York: Penguin (Non-Classics).

Lessig, Lawrence. 2006. Code: Version 2.0. New York: Basic Books.

Lessig, Lawrence. 2008. Remix: Making Art and Commerce Thrive in the Hybrid Economy. New York: Penguin.

Levy, Steven. 2007. “Are MySpace Users Now Spacing Out?” Newsweek, May 28. Retrieved December 3, 2008 (http://www.newsweek.com/id/34551).

Lewis, George H. 1990. "Community through Exclusion and Illusion: The Creation of Social Worlds in an American Shopping Mall." The Journal of Popular Culture 24(2): 121-136.

Li, Charlene. 2008. "Youth and Social Networks." From North American Social Technographics ${ }^{\circledR}$ Online Survey, Q2 2007; North American Technographics ${ }^{\circledR}$ Retail and Marketing Online Youth Survey, Q4 2007. Cambridge, MA: Forrester Research.

Lincoln, Sian. 2004. “Teenage Girls' 'Bedroom Culture’: Codes Versus Zones.” Pp. 94-106 in After Subculture: Critical Studies in Contemporary Youth Culture, edited by Andy Bennett and Keith Kahn-Harris. New York: Palgrave Macmillan.

Lincoln, Sian. 2005. "Feeling the Noise: Teenagers, Bedrooms and Music.” Leisure Studies 24(4): 399-414. 
Lipsman, Andrew. 2007. Social Networking Goes Global. comScore. Retrieved December 3, 2008 (http://www.comscore.com/press/release.asp?press=1555).

Lipsman, Andrew. 2008. Social Networking Explodes Worldwide as Sites Increase Their Focus on Cultural Relevance. comScore. Retrieved December 3, 2008 (http://www.comscore.com/press/release.asp? press=2396).

Liu, Hugo. 2007. “Social Network Profiles as Taste Performances.” Journal of Computer-Mediated Communication 13(1): article 13. Retrieved December 3, 2008 (http://jcmc.indiana.edu/vol13/issue1/liu.html).

Livingstone, Sonia M. 2002. Young People and New Media: Childhood and the Changing Media Environment. London, UK: Sage.

Livingstone, Sonia. 2005. Audiences and Publics: When Cultural Engagement Matters for the Public Sphere. Portland, OR: Intellect.

Livingstone, Sonia. 2006. "Children's Privacy Online: Experimenting with Boundaries Within and Beyond the Family.” Pp. 128-144 in Computers, Phones, and the Internet: Domesticating Information Technology, Human Technology Interaction, edited by Robert Kraut, Malcolm Brynin, and Sara Kiesler. Oxford, UK: Stanford University Press.

Livingstone, Sonia M. 2008. “Taking Risky Opportunities in Youthful Content Creation: Teenagers' Use of Social Networking Sites for Intimacy, Privacy and Self-Expression.” New Media \& Society 10(3): 459-477.

Lyall, Sarah. 2005. "What's the Buzz? Rowdy Teenagers Don't Want to Hear It.” The New York Times, November 29. Retrieved December 3, 2008 (http://www.nytimes.com/2005/11/29/international/europe/29repellent.html) 
Mahoney, Joseph L., Reed W. Larson, and Jacquelynne S. Eccles. 2005. Organized Activities as Contexts of Development: Extracurricular Activities, After School and Community Programs. Mahway, NJ: Lawrence Erlbaum.

Males, Mike and Dan Macallair. 1999. “An Analysis of Curfew Enforcement and Juvenile Crimes in California." Western Criminology Review 1(2). Retrieved December 3, 2008 (http://wcr.sonoma.edu/v1n2/males.html).

Malinowski, Bronislaw. 1922. Argonauts of the Western Pacific: An Account of Native Enterprise and Adventure in the Archipelagoes of Melanesian New Guinea. New York: Dutton.

Marcus, George E. 1995. "Ethnography in/of the World System: The Emergence of Multi-Sited Ethnography.” Annual Reviews in Anthropology 24: 95-117.

Marcus, George E. 1998. Ethnography through Thick and Thin. Princeton, NJ: Princeton University Press.

Markham, Annette N. and Nancy K. Baym. 2008. Internet Inquiry: Conversations about Method. London, UK: Sage.

Marlow, Cameron A. 2005. “The Structural Determinants of Media Contagion.” $\mathrm{PhD}$ Thesis, Media Arts and Sciences, Massachusetts Institute of Technology, Cambridge, MA.

Martin, Steven P. 2003. "Is the Digital Divide Really Closing? A Critique of Inequality Measurement in a Nation Online.” IT \& Society 1(4): 1-13.

Marwick, Alice. 2008. “To Catch a Predator? The MySpace Moral Panic.” First Monday 13(6): article 3. Retrieved December 3, 2008 (http://www.uic.edu/ htbin.cgiwrap/bin/ojs/index.php/fm/article/view/2152/1966). 
Matsuda, Misa. 2005. “Mobile Communication and Selective Sociality.” Pp. 123142 in Personal, Portable, Pedestrian: Mobile Phones in Japanese Life, edited by Mizuko Ito, Daisuke Okabe, and Misa Matsuda. Cambridge, MA: MIT Press.

McDougall, Bonnie S. and Anders Hansson, eds. 2002. Chinese Concepts of Privacy. Leiden, Netherlands: Brill.

McDowal, David, Colin Loftin, and Brian Wiersema. 2000. “The Impact of Youth Curfew Laws on Juvenile Crime Rates." Crime \& Delinquency 46(1): 76-91.

McLuhan, Marshall. 1964. Understanding Media: The Extensions of Man. New York: McGraw-Hill.

Mcnamara, Robert Hartmann. 2008. The Lost Population: Status Offenders in America. Durham, NC: Carolina Academic Press.

McPherson, Miller, Lynn Smith-Lovin, and James M. Cook. 2001. "Birds of a Feather: Homophily in Social Networks." Annual Review of Sociology 27(August): 415-444.

McRobbie, Angela and Jenny Garber. 1976. "Girls and Subcultures.” Pp. 209-222 in Resistance through Rituals: Youth Subcultures in Post-War Britain, edited by Stuart Hall and Tony Jefferson. New York: Routledge.

Mead, Margaret. 1928. Coming of Age in Samoa: A Psychological Study of Primitive Youth for Western Civilization. New York: W. Morrow.

Meyrowitz, Joshua. 1985. No Sense of Place: The Impact of Electronic Media on Social Behavior. New York: Oxford University Press.

Miller, Daniel and Don Slater. 2000. The Internet: An Ethnographic Approach. London, UK: Berg. 
Milner, Murray, Jr. 2004. Freaks, Geeks, and Cool Kids: American Teenagers, Schools, and the Culture of Consumption. New York: Routledge.

MissyChrissy. 2008. “Growing Up Online.” Families.com, February 1, 2008.

Retrieved December 3, 2008

(http://forums.families.com/showpost.php? $\mathrm{p}=908307$ \&postcount=9).

MissyChrissy. 2007. “My 15 yo son \& sex \& facebook.” Families.com, June 3, 2007.

Retrieved June 6, 2007

(http://forums.families.com/my-15-yo-son-amp-sex-amp-facebook,t108830).

Mitchell, William J. 1995. City of Bits: Space, Place, and the Infobahn. Cambridge, MA: MIT Press.

Momus. 1992. "Pop Stars? Nein Danke!” Grimsby Fishmarket. Retrieved December 3, 2008 (http://imomus.com/index499.html).

Montemayor, Raymond. 1983. "Parents and Adolescents in Conflict." Journal of Early Adolescence 3: 83-103.

Mosse, David. 2006. “Anti-Social Anthropology? Objectivity, Objection, and the Ethnography of Public Policy and Professional Communities*." Journal of the Royal Anthropological Institute 12(4): 935-956.

Muuss, Rolf E. 1995. Theories of Adolescence. Vol. 6. New York: Random House.

Negroponte, Nicholas. 1995. Being Digital. New York: Vintage Books.

Newcomb, Andrew F. and Catherine L. Bagwell. 1996. "The Developmental Significance of Children's Friendship Relations.” Pp. 289-321 in The Company They Keep: Friendship in Childhood and Adolescence, edited by William M. Bukowski, Andrew F. Newcomb, and Willard W. Hartup. Cambridge, UK: Cambridge University Press. 
Nissenbaum, Helen. 2004. "Privacy as Contextual Integrity." Washington Law Review 79(1): 101-139.

O’Shea, William. 2003. "Six Degrees of Sexual Frustration: Connecting the Dates with Friendster.com.” The Village Voice, July 4-10. Retrieved December 3, 2008 (http://www.villagevoice.com/news/0323,oshea,44576,1.html).

Ong, Walter J. 2002. Orality and Literacy. London, UK: Routledge.

Orgad, Shani. 2008. "How Can Researchers Make Sense of the Issues Involved in Collecting and Interpreting Online and Offline Data?” Pp. 32-53 in Internet Inquiry: Conversations about Method, edited by Annette N. Markham and Nancy K. Baym. Los Angeles, CA: Sage.

Ortiz, Steven M. 1994. "Shopping for Sociability in the Mall.” Pp. 193-199 in The Community of the Streets. Vol. Supplement 1, Research in Community Sociology, edited by Spencer E. Cahill and Lyn H. Lofland. Greenwich, CT: JAI Press.

Osgerby, Bill. 2004. Youth Media. London, UK: Routledge.

Osgood, D. Wayne and Amy L. Anderson. 2004. "Unstructured Socializing and Rates of Delinquency." Criminology 42(3): 519-550.

Pahl, Ray. 2000. On Friendship. Cambridge, UK: Polity.

Palfrey, John and Urs Gasser. 2008. Born Digital: Understanding the First Generation of Digital Natives. New York: Basic Books.

Parks, Malcolm R. and Kory Floyd. 1996. "Making Friends in Cyberspace.” Journal of Computer-Mediated Communication 1(4) Retrieved December 3, 2008 (http://jcmc.indiana.edu/vol1/issue4/parks.html). 
Pascoe, C. J. 2007. Dude, You're a Fag: Masculinity and Sexuality in High School. Berkeley, CA: University of California Press.

Paul, Smith Robert. 1957. Where Did You Go? Out. What Did You Do? Nothing. New York: Pocket Books.

Perkel, Daniel. 2006. "Copy and Paste Literacy: Literacy Practices in the Production of a MySpace Profile.” Presented at Informal Learning and Digital Media: Constructions, Contexts, Consequences, September 21-23, Denmark.

Perry, Pamela. 2002. Shades of White: White Kids and Racial Identities in High School. Durham, NC: Duke University Press.

Piacentini, Maria and Greig Mailer. 2004. "Symbolic Consumption in Teenagers' Clothing Choice." Journal of Consumer Behavior 3(3): 251-264.

Piaget, Jean. 1969. The Child's Conception of the World. Totowa, NJ: Littlefield, Adams, and Co.

Pimentel, Benjamin. 2006. "Keeping an Eye on the Kids: New Gadgets Leave Children Vulnerable in Cyberspace." San Francisco Chronicle, March 18. Retrieved December 3, 2008 (http://www.sfgate.com/cgi-bin/ article.cgi?f=/c/a/2006/03/18/BUG7VHQ3171.DTL).

Pinch, Trevor J. and Wiebe E. Bijker. 1984. "The Social Construction of Facts and Artefacts: Or How the Sociology of Science and the Sociology of Technology Might Benefit Each Other." Social Studies of Science 14(3): 399-441.

Pinch, Trevor J. and Wiebe E. Bijker. 1986. "Science, Relativism and the New Sociology of Technology: Reply to Russell." Studies of Science 16(2): 347-360.

Piot, Charles. 1999. Remotely Global: Village Modernity in West Africa. Chicago, IL: University of Chicago Press. 
Postman, Neil. 1994. The Disappearance of Childhood. New York: Vintage.

Reed, Adam. 2005. “'My Blog Is Me’: Texts and Persons in UK Online Journal Culture (and Anthropology)." Ethnos 70(2): 220-242.

Reid, Elizabeth M. 1991. "Electropolis: Communication and Community on Internet Relay Chat." Master's Thesis, Department of History, University of Melbourne, Melbourne, Australia.

Rettberg, Jill Walker. 2008. Blogging. Cambridge, UK: Polity Press.

Rheingold, Howard. 1994. The Virtual Community: Homesteading on The Electronic Frontier. New York: HarperPerennial.

Rheingold, Howard. 2002. Smart Mobs: The Next Social Revolution. Cambridge, MA: Perseus.

Rossler, Beate. 2004. The Value of Privacy. Cambridge, UK: Polity.

Ruefle, William and Kenneth Reynolds. 1995. "Curfew and Delinquency in Major American Cities." Crime and Delinquency 41: 347-363.

Russell, Stewart. 1986. "The Social Construction of Artefacts: Response to Pinch and Bijker." Social Studies of Science 16(2): 331-346.

Ryan, Mary P. 1992. Women in Public: Between Banners and Ballots, 1825-1880. Baltimore, MD: The Johns Hopkins University Press.

Savage, Jon. 2007. Teenage: The Creation of Youth Culture. New York: Viking.

Schrade, Brad. 2006. "Ex-Teacher Sent Teen Naked Pics." The Tennessean, April 27. Retrieved December 3, 2008 (http://www.tennessean.com/apps/pbcs.dll/ article?AID=/20060427/NEWS0201/604270438). 
Senft, Theresa M. 2008. Camgirls: Celebrity and Community in the Age of Social Networks. New York: Peter Lang.

Shea, Danny and Matt Feinstein. 2006. "An Open Letter to Mark Zuckerberg.” Daily Princetonian, March 9. Retrieved December 3, 2008 (http://www.dailyprincetonian.com/archives/2006/03/09/arts/14810.shtml).

Shepheard, Paul. 1994. What Is Architecture? An Essay on Landscapes, Buildings, and Machines. Cambridge, MA: The MIT Press.

Shirky, Clay. 2008. Here Comes Everybody: The Power of Organizing without Organizations. New York: Penguin.

Simmel, Georg. 1972. On Individuality and Social Forms. Chicago, IL: University of Chicago Press.

Skelton, Tracey and Gill Valentine. 1998. Cool Places: Geographies of Youth Cultures. London, UK: Routledge.

Solove, Daniel. 2007. “'I've Got Nothing to Hide'and Other Misunderstandings of Privacy." San Diego Law Review 44: 745.

Solove, Daniel J. 2006. The Digital Person: Technology and Privacy in the Information Age. New York: NYU Press.

Solove, Daniel J. 2007. The Future of Reputation: Gossip, Rumor, and Privacy on the Internet. New Haven, CT: Yale University Press.

Soukup, Charles. 2006. "Computer-Mediated Communication as a Virtual Third Place: Building Oldenburg's Great Good Places on the World Wide Web.” New Media \& Society 8(3): 421.

Springhall, John. 1998. Youth, Popular Culture and Moral Panics. New York: St. Martin's Press. 
Starr, Paul. 2005. The Creation of the Media: Political Origins of Modern Communication. New York: Basic Books.

Steele, Jeanne R. and Jane D. Brown. 1995. “Adolescent Room Culture: Studying Media in the Context of Everyday Life." Journal of Youth and Adolescence 24(5): 551-576.

Steiner, Peter. 1993. “On the Internet, Nobody Knows You're a Dog.” The New Yorker, 69(20): 61.

Stern, Susannah R. 2004. "Studying Adolescents Online: A Consideration of Ethical Issues.” Pp. 274-287 in Readings in Virtual Research Ethics: Issues and Controversies, edited by Elizabeth A. Buchanan. Hershey, PA: Information Science.

Sternheimer, Karen. 2006. Kids These Days: Facts and Fictions about Today's Youth. Lanham, MD: Rowman and Littlefield.

U.S. Congress. Senate. Protecting Children in the 21st Century Act. S. 49. 110th Congress, 2007.

Stone, Allucquère Rosanne. 1995. The War of Desire and Technology at the Close of the Mechanical Age. Cambridge, MA: MIT Press.

Strauch, Barbara. 2003. The Primal Teen: What the New Discoveries about the Teenage Brain Tell Us about Our Kids. New York: Doubleday.

Subrahmanyam, Kaveri and Patricia Greenfield. 2008. "Online Communication and Adolescent Relationships.” The Future of Children 18(1): 119-146.

Sundén, Jenny. 2003. Material Virtualities: Approaching Online Textual Embodiment. New York: Peter Lang. 
Swidey, Neil. 2008. "Friends in a Facebook World.” Boston Globe, November 30.

Retrieved December 3, 2008 (http://www.boston.com/bostonglobe/magazine/ articles/2008/11/30/friends_in_a_facebook_world/).

Tapscott, Don. 1998. Growing Up Digital: The Rise of the Net Generation. New York: McGraw-Hill.

Tapscott, Don and Anthony D. Williams. 2006. Wikinomics: How Mass Collaboration Changes Everything. New York: Portfolio.

Taylor, T. L. 2006. Play between Worlds: Exploring Online Game Culture. Cambridge, MA: MIT Press.

Thelwall, Michael. 2008. "Fk yea I swear: Cursing and Gender in MySpace." Corpora 3(1) 83-107.

Thompson, Michael, Catherine O’Neill Grace, and Lawrence J. Cohen. 2001. Best Friends, Worst Enemies: Understanding the Social Lives of Children. New York: Ballantine Books.

Thorne, Barrie. 1993. Gender Play: Girls and Boys in School. New Brunswick, NJ: Rutgers University Press.

Thornton, Sarah. 1996. Club Cultures: Music, Media, and Subcultural Capital. Middletown, CT: Wesleyan University Press/University Press of New England.

Toch, Hans. 1992. Living in Prison: The Ecology of Survival. Washington, DC: American Psychological Association (APA).

Turkle, Sherry. 1984. The Second Self: Computers and the Human Spirit. New York: Simon and Schuster. 
Turkle, Sherry. 1995. Life on the Screen: Identity in the Age of the Internet. New York: Simon and Schuster.

Turner, Fred. 2006. From Counterculture to Cyberculture: Stewart Brand, the Whole Earth Network, and the Rise of Digital Utopianism. Chicago, IL: University of Chicago Press.

Turner, Graeme. 2004. Understanding Celebrity. London, UK: Sage.

Ungar, Michael. 2004. “The Importance of Parents and Other Caregivers to the Resilience of High-risk Adolescents.” Family Process 43(1): 23-41.

Valentine, Gill. 2004. Public Space and the Culture of Childhood. Hants, UK: Ashgate.

van Dijk, Jan and Kenneth Hacker. 2003. "The Digital Divide as a Complex and Dynamic Phenomenon." The Information Society 19(4): 315-326.

Various. 2008. “How Much Privacy Do You Give a Teen?” Askville, January 25, 2008. Retrieved December 3, 2008 (http://askville.amazon.com/ privacy-give-teen/AnswerViewer.do? requestId=7624995).

Wakeford, Nina. 2003. "Internet Cafés in London: The Embedding of Local Culture in Global Communication: Independent.” New Media \& Society 5(3): 379399.

Walker, Jesse. 2004. Rebels on the Air: An Alternative History of Radio in America. New York: NYU Press.

Warner, Michael. 2002. Publics and Counterpublics. Cambridge, MA: MIT Press.

Wartella, Ellen and Sharon Mazzarella. 1990. “A Historical Comparison of Children's Use of Leisure Time." Pp. 173-194 in For Fun and Profit: The 
Transformation of Leisure into Consumption, edited by Richard Butsch. Philadelphia, PA: Temple University Press.

Weinberger, David. 2002. Small Pieces Loosely Joined: A Unified Theory of the Web. Cambridge, MA: Perseus.

Weinberger, David. 2008. Everything Is Miscellaneous: The Power of the New Digital Disorder. New York: Holt Paperbacks.

Willis, Paul. 1981. Learning to Labor: How Working Class Kids Get Working Class Jobs. New York: Columbia University Press.

Wilson, Brian. 2006. "Ethnography, the Internet, and Youth Culture: Strategies for Examining Social Resistance and 'Online-Offline' Relationships.” Canadian Journal of Education 29(1): 307-328.

Wolak, Janis, David Finkelhor, Kimberly J. Mitchell, and Michele L. Ybarra. 2008. "Online 'Predators' and Their Victims: Myths, Realities, and Implications for Prevention and Treatment." American Psychologist 63(2): 111-128.

Wolak, Janis, Kimberly Mitchell, and David Finkelhor. 2003. “Escaping or Connecting? Characteristics of Youth Who Form Close Online Relationships.” Journal of Adolescence 26: 105-119.

Wolak, Janis, Kimberly Mitchell, and David Finkelhor. 2006. Online Victimization of Youth: Five Years Later. National Center for Missing and Exploited Children. Retrieved December 3, 2008 (http://www.unh.edu/ccrc/pdf/CV138.pdf).

Wolak, Janis, Kimberly Mitchell, and David Finkelhor. 2007. “Does Online Harassment Constitute Bullying? An Exploration of Online Harassment by Known Peers and Online-Only Contacts." Journal of Adolescent Health 41: S51-S58. 
Wright, Frank Lloyd and Frederick Ed Gutheim. 1941. On Architecture: Selected Writings (1894-1940). New York: Grosset and Dunlap.

Ybarra, Michele and Kimberly Mitchell. 2004. "Youth Engaging in Online Harassment: Associations with Caregiver-Child Relationships, Internet Use, and Personal Characteristics." Journal of Adolescence 27: 319-336.

Ybarra, Michele L. and Kimberly Mitchell. 2008. "How Risky Are Social Networking Sites? A Comparison of Places Online Where Youth Sexual Solicitation and Harassment Occurs." Pediatrics 121(2): E350-E357.

Zittrain, Jonathan. 2008. The Future of the Internet-And How to Stop It. New Haven, CT: Yale University Press.

Zuckerman, Ethan. 2008. "What Bloggers Amplify from the BBC." My Heart's in Accra, January 28, 2008. Retrieved December 3, 2008 (http://www.ethanzuckerman.com/blog/2005/01/28/what-bloggers-amplifyfrom-the-bbc/). 


\section{Appendix 1: Brief Descriptions of Teens}

This Appendix contains brief descriptions of the teens mentioned throughout this dissertation. It includes both teens that I interviewed and teens that I observed. Not all teens that I interviewed are listed, although their demographics are described in aggregate in Chapter 2. The purpose of this Appendix is to shed more light on the quoted teens. My descriptions vary-some focus on physical characteristics or how the teen identifies while others focus on familial dynamics or hobbies. In choosing what to describe, I focus on what was most salient and may provide context for the quotes included.

I name cities when teens live in one of the 50 most populous cities in the United States; otherwise, I name states. Teens who I interviewed identified their age, grade, and race or ethnicity on a form before the interview. Gender was an open-ended question, but I converted markings like "F" or "girl" to female and "M" or "guy" to male. No subject identified as transgender on either the form or during the interview. I do not always have complete demographic information for teens whom I observed online. Where possible, I provide information that I can discern from their profiles. The ages provided are based on the date of their interview or online content.

In marking race and ethnicity, I draw on what teens marked on the form I provided and the way they identified in the interview or on their profile. Where 
multiple markers are provided, teens identified both; I list these alphabetically. I also use the most specific term teens used (e.g., Guatemalan instead of Latina).

Except when last names are provided, all names are pseudonyms. Those teens identified by first and last name either requested to be identified as such or published content for mass consumption. In many cases, teens chose their own pseudonyms; I selected the rest using a baby-name site.

\section{Aaron (15, Texas): $9^{\text {th }}$ grade, white, male.}

Interview: March 14, 2007.

Aaron, from suburban Austin, is far more interested in video games, making videos, and playing pranks than in girls. Fred is a close friend and they hang out a lot. His mother does not work and is generally concerned about him and his older sister. He does not tell her a lot of what he does so as to not worry her. He is involved in church and appears to be a fairly good student. SNS: MySpace (without parental knowledge).

\section{Alexis (16, Seattle): $10^{\text {th }}$ grade, Latina/white, female.}

Interview: January 20, 2007.

Alexis is a scrawny girl with bleached blond hair that is growing out. She is a vegetarian and likes to party a lot. Her mother has a problem with meth and is not that present as a mother. Alexis uses MySpace to find out what is happening and 
enjoys going out as much as possible. School is not her thing and her fashion suggests that she is into the indie rock subculture. She is a close friend of Kira's, although they rarely get together because of Kira's restrictions. SNS: MySpace.

\section{Allie (17, Indiana): $12^{\text {th }}$ grade, white, female.}

MySpace: December 7, 2007.

Allie's online identity is heavily shaped by her Christian views. Each blog post references God and she quotes scripture throughout her profile. She has a simple profile; she has not modified the layout. She comments regularly with a small number of friends and writes long, reflective blog posts, focused primarily on her struggles with religion. Allie is a redhead with a pixie haircut and, after turning 18, she posted photos of a new nose ring and a tattoo with a scripture reference. SNS: MySpace.

\section{Amy (16, Seattle): $10^{\text {th }}$ grade, black/white, female.}

Interview: January 20, 2007.

Amy is sociable, but her parents are rather strict and rarely allow her to socialize with friends outside of school. She was dating Michael at the time of our interview, but they broke up shortly afterward. She has long, straight, dark hair and is very expressive when she talks, but she also lets Michael speak for her. In her profile, she identifies boys, music, MySpace, and her cell phone as being of key importance to her and her photos highlight her relationship with friends. SNS: MySpace. 


\section{Ana-Garcia (15, Los Angeles): $10^{\text {th }}$ grade, Guatemalan/Pakistani, female.}

Interview: March 5, 2007.

Ana-Garcia lives with her parents, baby sister, and two brothers. Her family is Muslim and her father is quite strict with her, not allowing her to do much for fear that she will "run with boys" and get herself into trouble. Her father does not like her friends and does not let her hang out with them. For the most part, Ana-Garcia is unable to leave the house except to go to religious-based activities, although her mother sometimes allows her without telling her father. She is not allowed to be involved in after-school activities, either. While her brothers can do as they please, she is expected to stay at home and help her mother. SNS: MySpace.

\section{Anastasia (17, New York): $12^{\text {th }}$ grade, female.}

Comments: August 11, 2007.

Anastasia left a series of comments on my blog after her sister forwarded my essay on class dynamics in MySpace and Facebook to her. She mapped out the dynamics in her own school in a suburb of Manhattan and explained why social network sites were important to her and her peers; she encouraged other teenagers to join the dialogue and voice their opinions. She identified herself as "one of those kids that teachers and parents alike just love." In some of her comments, she chastised adults for not understanding teen life, mocked her peers for their immaturity, criticized MySpace for its advertising content, and encouraged parents to talk with their kids. SNS: Facebook, MySpace. 


\section{Anindita (17, Los Angeles): $12^{\text {th }}$ grade, Indian, female.}

Interview: February 20, 2007.

Anindita sees herself as an excellent student, popular in her school, and well liked by teachers. She is the only child of immigrant Indian parents whom she seeks to please. Her parents are restrictive, but she understands and appreciates this. SNS: Facebook, MySpace.

\section{Ann (15, Seattle): $9^{\text {th }}$ grade, white, female.}

Interview: January 21, 2007.

Ann's father is a pastor. She attends church every weekend and is raising money for the church. Every other weekend, she attends her friend Natalie's church because she does youth group with Natalie. She is very mild mannered, plays soccer and runs track, and babysits. She is terrified of predators' talking to her and her MySpace location is "Not Talking to Stalkers." SNS: MySpace.

\section{Anthony (14, Pennsylvania): $9^{\text {th }}$ grade, white, male.}

Interview: May 5, 2007.

Anthony likes skateboarding with his friends and is involved in various sports and activities. He does not spend much time online because he prefers to be doing things. He makes videos with his friends, but he has never uploaded them. Emily is his sister; their social worlds are very separate. SNS: MySpace. 


\section{Aria (20, California): college student, female.}

Blog: October 27, 2007.

Aria is a humanities major at a public college in Northern California. She is also a competitive cyclist and has a sharp sense of humor in her writing. Aria is not currently a U.S. citizen, but she has applied for citizenship and is waiting for the paperwork to go through. She has traveled extensively, including cycling in Kenya. SNS: MySpace.

\section{Bianca (16, Michigan): $10^{\text {th }}$ grade, white, female.}

Interview: June 26, 2007.

Bianca is a quiet girl with a handful of friends. She enjoys school. She has two younger sisters and her mother is a "homemaker." She and Sasha are best friends and neighbors; they hang out at Sasha's house frequently. Bianca babysits for money and regularly attends church, although she prefers Sasha's church and youth group to the one her parents attend. She loves text messaging. SNS: Facebook.

\section{Bly Lauritano-Werner (17, Maine): $12^{\text {th }}$ grade, white, female.}

Youth Radio Story: July 24, 2006.

Bly wrote and produced a story for Youth Radio about her struggles with her mother over her online diary. Her story, "Reading My LiveJournal" can be heard at 
http://www.youthradio.org/society/npr060628_onlinejournal.shtml—the story includes both her perspective and that of her mother. SNS: Facebook, LiveJournal.

\section{Brooke (15, Nebraska): $9^{\text {th }}$ grade, white, female.}

Interview: April 17, 2007.

Brooke has two close friends with whom she primarily socializes. Church youth group is really important to her. Her parents' house is solidly middle class and decorated in colonial style. She lives with her parents and her younger brother. Her parents have many rules, including a non-homework computer limitation of 30 minutes per day. Her mother regularly makes snacks for the neighborhood kids and socializes with the other parents in the neighborhood. She is shy and uncomfortable talking about herself; she prefers talking to people through the computer than over the phone. SNS: Facebook.

\section{Cachi (18, Iowa): $12^{\text {th }}$ grade, Puerto Rican, female.}

Interview: April 18, 2007.

Cachi is an outgoing girl with a lot of confidence and sass. Her mother left her when she was young, but they recently reconnected and she now lives in a generic new suburban neighborhood with her mother, her stepfather, and four of her siblings. She has five siblings and takes care of the three younger ones; she jokes that she also takes care of her mother, especially when her mother has been drinking. She 
reads as older than her age and has no problem getting into bars. She used to date Stan and is friends with Red and Wolf. SNS: Facebook, MiGente, MySpace.

\section{Cara (20, Maine): college student, female.}

Blog: August 21, 2006.

Cara is a bit of a social outcast and jokes that she is emo. She maintains a LiveJournal and is part of an online community dedicated to androgynous and boyish women. All quotes from Cara come from her LiveJournal. SNS: LiveJournal, MySpace.

Catalina (15, Austin): $10^{\text {th }}$ grade, white, female.

Interview: March 14, 2007.

Catalina lives in a traditional suburban neighborhood with her parents and two siblings. She is active in many activities, including track and drama. She and Jordan are friends, but they are also part of a broader friend group that socializes together regularly when they have spare time. She goes to a private Catholic school and attends church. SNS: Facebook, MySpace.

\section{Christopher (15, Alabama): between $9^{\text {th }}$ and $10^{\text {th }}$ grade, white, male.}

Interview: June 27, 2007.

Christopher's parents are divorced. During the school year, he lives with his mother, stepfather, two brothers, and a half sibling. He spends the summers in 
Michigan with his father, stepmother, brothers, and two other half siblings. In Alabama, Christopher has an extensive network of friends, primarily from church, but in Michigan, he knows no one his age and his family does not attend church. In Alabama, he plays music and does a lot of volunteering through church. In Michigan, his stepmother got him a job investigating Teen Second Life. He is into videogames and the computer more generally. SNS: None due to lack of interest.

\section{Clyde (16, Michigan): between $10^{\text {th }}$ and $11^{\text {th }}$ grade, Hispanic/white, male.}

Interview: June 26, 2007.

Clyde lives in a small house with his parents, brother, and two large dogs. His grandparents live nearby and he is quite close to them, especially his grandmother, who drives him places. His father does not work and it is clear that money is an issue in his family; lack of economic resources regularly emerges as an explanation for why he cannot do different things. That said, Clyde is involved in the school band and theater, almost an Eagle Scout, and passionate about video games. In general, Clyde is very negative and speaks derogatively about his classmates and life. SNS: Facebook, MySpace.

\section{Court (17, Nebraska): $11^{\text {th }}$ grade, white, male.}

Interview: April 27, 2007.

Court works at his father's greenhouse after school and on weekends. He is not that technologically engaged and gets online only every week or so. While the 
majority of his classmates use MySpace, Court has seen the site but has no interest in interacting with people online. He used to use IM, but no longer. His Internet use is primarily about downloading music and occasionally watching videos. To connect with friends, Court primarily uses his cell phone. SNS: None due to lack of interest.

\section{Craig Pelletier (17, California): $12^{\text {th }}$ grade, male.}

Blog: February 10, 2008.

Craig's father forwarded him my essay on class dynamics in MySpace and Facebook and this prompted him to write a blog post titled "Myface; Spacebook" about the dynamics he witnessed in his school. Craig's blog is a mix of social commentary and storytelling and the Wordpress design suggests that he is comfortable with technology. SNS: Facebook, MySpace.

\section{Dan (15, California): $10^{\text {th }}$ grade, white, male.}

Online conversations: Fall 2006.

Dan and I met at a conference for hackers, geeks, and techies. He lives in a small town in Northern California with his parents and attends a high school that focuses on technology. He enjoys helping people understand emergent technologies and he interns for a company doing precisely that. SNS: Facebook, MySpace. 


\section{Danny (17, Florida): $11^{\text {th }}$ grade, white, male.}

Email: March 26, 2006.

Danny is a gay teen living in what he describes as a "horrible red state." He is an active user of different social media and it appears that he uses the Internet to make connections with people who share his views. He read about me in Wired and emailed me a long note describing his opinion on online safety issues with respect to teens. Danny's native language is French and he blogs regularly about a wide range of topics but most notably gay rights issues. As a hobby, he does voices for friends' films. SNS: DeviantArt, Facebook, MySpace.

\section{Denzel (16, Los Angeles): $11^{\text {th }}$ grade, black, ${ }^{15}$ male.}

Interview: January 12, 2007.

Denzel is Nick's fraternal twin brother. His family is very close. He has an 11year-old sister of whom he and his brother are very protective. His father is the football coach at his school and Denzel is on the team. His father also has a MySpace, which he uses to talk to the football team. Denzel loves his Sidekick and uses it frequently to IM with friends. He is a strong student and is hoping to go to UCLA and become a civil engineer. SNS: MySpace.

\footnotetext{
${ }^{15}$ Denzel self-identifies as black; his twin brother Nick self-identifies as black with Native American roots.
} 


\section{Dom (16, Washington): $10^{\text {th }}$ grade, black, male.}

Interview: January 21, 2007.

Dom is an avid Internet user and uses a wide array of social media. He is passionate about hip-hop and rap music and enjoys making his own tracks with friends. He uses the Internet to connect with other musicians. On his MySpace, he lists his ex-girlfriend as his \# 1 because they are still friends. Dom is close with his brother Jon, who is one year older. SNS: MySpace.

\section{Dominic (16, Seattle): $10^{\text {th }}$ grade, white, male.}

Interview: January 21, 2007.

Dominic is calm, centered, and articulate. His passions are primarily interestdriven and somewhat geeky. Unlike his close friends (Mark and Kevin), he does not play World of Warcraft any longer because he cannot afford it; he does like other video games and plays them regularly. He also likes to draw and practices martial arts. SNS: Facebook, MySpace.

Eduardo (17, Los Angeles): high-school dropout, Hispanic, male.

Interview: December 5, 2006.

Eduardo got kicked out of high school, although he is starting to work with the alternative school to get a GED or high-school diploma. He lives with his mother and does not know his father. He knows of eight siblings, but he lives with only two 
of them and has never met one of them. Eduardo is passionate about making rap music and goes to the anti-gang community center to make music. He also likes basketball and photography and he wants to either be a photographer or a police officer. SNS: MySpace.

\section{Emily (16, Pennsylvania): $10^{\text {th }}$ grade, white, female.}

Interview: May 5, 2007.

Emily is not particularly popular and has struggled with this for a while; she is not particularly into school either. She prefers hanging out with peers in groups to doing things in smaller intimate groups. What she does is very influenced by her

peers and she knows it. Her passion is hunting. Anthony is her brother; their social worlds are very separate. SNS: Xanga.

\section{Fred (15, Texas): $9^{\text {th }}$ grade, white, male.}

Interview: March 14, 2007.

Fred, from suburban Austin, is a slightly geeky boy who really likes games and technology. His parents restrict his access to the Internet because they think that it takes away from his homework. In the summer, he will get back to playing World of Warcraft. He is far more interested in video games, making videos, and playing pranks than in girls. He is close with Aaron and they get together a lot. He is involved in his church and goes to church camp in the summers. SNS: None due to parental restriction. 


\section{Gabriella (15, Los Angeles): $10^{\text {th }}$ grade, Honduran, female.}

Interview: January 23, 2007.

Gabriella is an attractive girl and she knows it. She is wearing full makeup, her fingernails are all done up, and she talks in a way that makes it clear that she is used to charming everyone. Boys, shopping, and socializing are the center of her universe. Lolo acts as her sarcastic sidekick, constantly teasing Gabriella. She dates constantly, but her boyfriends tend to be jealous and controlling, restricting what she can do online and watching over her shoulder. She is regularly in trouble with her mother, mostly because she is not supposed to be dating. When she's "on punishment," her mother takes away her access to social technologies. SNS: MySpace.

\section{Gadil (16, Los Angeles): $11^{\text {th }}$ grade, Indian, male.}

Interview: March 5, 2007.

Gadil is a hard-working student taking all advanced placement classes in a magnet school for students interested in math, science, and engineering. He is involved in many activities, but tennis, yearbook, and the South Asian Club are the most important to him. He lives with his mother, three younger siblings, grandparents, and aunt. They speak Urdu and English at home. SNS: Facebook, LiveJournal, MySpace, Xanga. 


\section{Heather (16, Iowa): $10^{\text {th }}$ grade, white, female.}

Interview: April 21, 2007.

Heather's passion is swimming-her school year and summers revolve around swimming. Her friends are mostly from swim team, swim camp, or are swimmers at other schools. She works part time at Panera Bread. She drives and this gives her a great deal of freedom. She is quite confident, bouncy, and outgoing. She is in AP classes in school. She lives with her parents and a foreign-exchange student; her brother goes to college. SNS: Facebook, MySpace, Xanga.

\section{Hollie (15, Pennsylvania): $10^{\text {th }}$ grade, white, female.}

Interview: May 5, 2007.

Hollie is passionate about music and theater and dresses in emo fashion (think black eyeliner, T-shirts from Hot Topic). She lives in a small town in an old house that is filled with tchotchkes. Hollie is active in her church's youth group, does mission work during the summers, and is dating a boy from church. She is very focused on her friends and uses social media to communicate with them often. Her mother forbids her to use sites like MySpace, but she does so anyhow so that she can talk to friends from school and church. SNS: MySpace, Xanga (formerly). 
James (17, Seattle): $11^{\text {th }}$ grade, white with Native American roots, male.

Interview: January 20, 2007.

James is a bit cocky and enjoys ranting with sarcastic overtones. He is a plainlooking boy with closely cropped dirty blond hair who rarely smiles. He appears to be a bit of an outcast, but he is friends and neighbors with Michael. He is not that interested in school but really likes technology. His parents give him tremendous freedom. James is also passionate about cars and snowboarding. On MySpace, he lists himself as a drug dealer who makes more than $\$ 250,000$; he finds this entertaining. SNS: MySpace.

Jennifer (17, Kansas): $11^{\text {th }}$ grade, white, female.

Interview: April 15, 2007.

Jennifer is into country music, works at a grocery store chain, and is active in church. She is involved in school and participates in a variety of activities, including a marketing group and Young Life. Jennifer lives with her aunt and uncle and her cousins (whom she calls siblings). Her mother made some bad decisions, which is why she does not live with her, but they are friends and get closer as they get older. Her clothing style and crooked teeth signal that she comes from a poor background. She has a car and spends a lot of her money from work on gas. She is afraid of MySpace. She and Julie are good friends. SNS: Facebook. 
Jessica (14, Massachusetts): $9^{\text {th }}$ grade, white, female.

Interview: June 20, 2007.

Jessica is quiet but talkative, motivated by school, learning, and eventually going to college. She has gone to different schools but likes the charter school she is in now because she has more opportunities to be involved in activities and to be a part of student government. She uses IM, texting, and Facebook to connect with friends. SNS: Facebook.

Jill (14, Los Angeles): $9^{\text {th }}$ grade, El Salvadoran, female. Interview: January 23, 2007.

Jill is reserved around me but much more outgoing among her peers. She is quite active on MySpace-her background depicts a designer label's purses and her profile is covered with images of friends. She lives with her parents and one of her three older brothers. She goes to church on Fridays and Sundays and spends the rest of the weekend with her family. Her cousin is her closest friend, but she has a broad network at school and church. SNS: MySpace.

\section{Jordan (15, Austin): $10^{\text {th }}$ grade, Mexican/white, female.}

Interview: March 14, 2007.

Jordan is an only child, her mother is from Mexico, and her dad is from Iowa; they speak Spanish and English at home. She's involved in numerous activities, 
including soccer and church. She and Catalina are good friends and go to a small Catholic school. They are part of a much bigger group that they regularly socialize with, but spare time is definitely an issue. SNS: Facebook, MySpace.

\section{Julie (17, Kansas): $11^{\text {th }}$ grade, white, female.}

Interview: April 15, 2007.

Julie lives in a rural part of Kansas and has chickens, dogs, and cats. Julie is quiet, preppy, a cheerleader, and generally a "good girl." She wants to be a dental hygienist and move to Dallas because she loves the Dallas Cowboys and because it is big and appealing. She has a car, which is good because her closest friend is more than 10 minutes away by car. She has an older brother and lives with her parents. She is into country music and afraid of MySpace. She and Jennifer are good friends. SNS: Facebook.

Kaleb (15, Michigan): between $9^{\text {th }}$ and $10^{\text {th }}$ grade, black, male. Interview: June 27, 2007.

Kaleb is really into video games, music, and the black student union at school. He goes to school near his mother's work rather than in his neighborhood because it is a more diverse school. He bikes to his mother's office afterward and roams the campus or goes to a nearby community center. He lives with his parents and younger brother. SNS: Facebook, MySpace. 


\section{Kat (15, Massachusetts): $9^{\text {th }}$ grade, white, female.}

Interview: June 20, 2007.

Kat attends a charter school and there are only 30 people in her class. Kat wants to go to Princeton. She is extremely involved in activities, loves dance and has been dancing since she was young, and does lots of community service. She attends summer camp, likes to read, and is generally engaged. She has an older sister who goes to a different school. SNS: Facebook, MySpace (formerly).

\section{Keke (16, Los Angeles): $11^{\text {th }}$ grade, black, female.}

Interview: January 12, 2007.

Keke's world revolves around money and shopping; she is obsessed with material objects as a marker of status. She has many siblings older and younger; her older brother was recently killed in a gang fight. She is not that much into MySpace but her friends are and they created a profile for her. She prefers writing, but she does not share her thoughts with anyone. SNS: MySpace.

\section{Kevin (15, Seattle): $10^{\text {th }}$ grade, white, male.}

Interview: January 20, 2007.

Kevin is sweet and reads as a classic dork. He is a little chunky in that way that suggests he does not exercise, although he does play football. His MySpace is covered with girls, but it is clear that he is not yet dating. He is friends with Dominic and 
Mark, and the three of them live near each other. He does not have a broader friend group. He really likes video games and, particularly, World of Warcraft. SNS:

Facebook, MySpace, Xanga.

\section{Kiki (16, Kansas): $10^{\text {th }}$ grade, black/white, female.}

Interview: April 15, 2007.

Kiki lives with her mother's best friend; her mother died two years ago and her father is in prison. She spends a lot of time at a youth center because it gives her the freedom to hang out with friends without anyone's worrying. Her phone is really important to her, but she does not spend much time online because she prefers to go to the youth center. Kiki has a nose ring and big earrings; she is heavyset and has her hair partially twisted. She is outgoing and friends are tremendously important to her. SNS: MySpace.

\section{Kira (17, Seattle): $12^{\text {th }}$ grade, Latina/white, female.}

Interview: January 21, 2007.

Kira lives with her very strict aunt, her cousin, and her uncle; two of her stepsisters are in foster care. Her mother recently remarried and she and her new husband have twin girl babies; I get the sense that the new stepfather did not want former children around. Kira's got various piercings and dresses like a cross between a raver and a hippie. She is not allowed to do much-her aunt does not let her leave 
the house and her grandfather is convinced that she is going to "fuck up" like her mother. SNS: MySpace.

\section{Laura (17, Washington): $12^{\text {th }}$ grade, white with Native American roots, female.}

Interview: January 20, 2007.

Laura goes to Catholic school, but she is frustrated by the lack of religious values in her peers. Christianity is central to her identity and her MySpace profile reflects this. She lives an hour outside of Seattle in a small town; her parents are divorced and she moves between them. She identifies herself as a nerd because she likes to read. She created her MySpace to keep in touch with friends from different schools. Laura's boyfriend is jealous when she communicates with other boys online. SNS: MySpace.

\section{Leigh (14, Iowa): $9^{\text {th }}$ grade, white, female.}

Interview: April 22, 2007.

Leigh identifies as a lesbian and has been involved in her school's GSA since the beginning. She dated a girl whom she met through a school trip, but while they talked online regularly, they only met in person twice. She is passionate about the environment and gets involved with different activities, mostly because of parental encouragement. She goes to church every weekend, but she does not like it. She is not interested in socializing with the popular people at school. She has an older sister 
and lives with her parents. She and Myra are close friends, although they do not have much time to hang out outside of school these days. SNS: MySpace.

\section{Lila (18, Michigan): between $12^{\text {th }}$ grade and college, Vietnamese, female.}

Interview: June 27, 2007.

Lila is extremely bubbly and sociable. She lightens up when talking about friends or shopping. She is about to go to college nearby, but she will not live at home. Tara is her younger sister and she has another sister who is much older. She and Tara are very close and they finish each other's sentences, share clothes, and share most interests. Lila has a car and is given tremendous freedom. SNS: Facebook.

\section{Lilly (16, Kansas): $10^{\text {th }}$ grade, white, female.}

Interview: April 16, 2007.

Lilly writes for the school paper, plays soccer, and has aspirations of going to Cornell to do medicine or journalism. She lives with her mother and stepfather and has one sibling and two half siblings. Lilly shares her mother's desire to save the world and is passionate about being "productive." She and Melanie are good friends and live near each other. They used to hang out more before sports took over their lives. SNS: Facebook, MySpace. 


\section{Lolo (15, Los Angeles): $10^{\text {th }}$ grade, Guatemalan, female.}

Interview: January 23, 2007.

Lolo is Gabriella's friend and snarky sidekick. She is a bit heavier and less done up. She is also into boys, shopping, and socializing, but she does not get into nearly as much trouble as Gabriella does. She has a boyfriend, but her relationships do not seem to last long. Her ex-boyfriend made her delete her MySpace because of the drama it caused; she has not re-created it, although Gabriella is pressuring her to do so. Her parents are relatively strict, but she navigates this without a problem. SNS: None due to pressure from ex-boyfriend.

Maria (15, Massachusetts): $9^{\text {th }}$ grade, Peruvian, female.

Interview: June 20, 2007.

Maria was born in Peru but was adopted by a single woman shortly after she was born. She lives with her mother and has three younger sisters, ages 11,8 , and 4 . She and her mother are very close and her friends love her mother. Maria wants to be more involved in sports, but her small charter school does not offer them so she intends to switch schools next year. SNS: Facebook. 
Mark (15, Seattle): $10^{\text {th }}$ grade, white, male.

Interview: January 21, 2007.

Mark is extremely negative, especially toward his peers. He does not have many friends other than Kevin and Dominic, nor does it appear that he makes an effort to make more friends. He is relatively geeky, very aware of Web2.0 technologies, and likes playing World of Warcraft. He maintains multiple MySpaces and enjoys the technical aspects of MySpace. SNS: Facebook, MySpace.

\section{Martin (17, Ontario): $12^{\text {th }}$ grade, white, male.}

Blog: July 16, 2007.

Martin lives just over the Canadian border outside of Michigan. He is gay and uses the Internet to find other gay and gay-friendly teens. He loves video games and science-fiction TV shows. He wants to write plots for video games. Martin is short and skinny. SNS: Facebook, MySpace.

\section{Melanie (15, Kansas): $10^{\text {th }}$ grade, white, female.}

Interview: April 16, 2007.

Melanie is a photojournalist and works at the school paper. She also swims and loves Harry Potter (but not as a fan fic type). She keeps a diary that she writes in every night. She lives with her mother (who is single and dating a lot); her 18-yearold brother lives with her father, who is not far away. She is close to both of them. 
She is close friends with Lilly and they live nearby. Sports have taken over their lives so they do not spend much time together. SNS: Facebook, MySpace.

\section{Mic (15, Los Angeles): $10^{\text {th }}$ grade, Egyptian, male.}

Interview: January 22, 2007.

Mic is the only son of immigrant parents. They speak Arabic at home. His father is tremendously strict and believes that Mic is not mature enough to use the Internet so there is no access at home. He is also not allowed to socialize with peers and his father brings him home during lunch and immediately after school. He does socialize with cousins during family gatherings and with peers at church, which his family attends religiously. He likes technology, is not that good at school, and seems relatively numb. SNS: None due to parental restrictions.

\section{Michael (17, Seattle): $11^{\text {th }}$ grade, white, male.}

Interview: January 20, 2007.

Michael is a skinny teen with a mustache and plays football and soccer. He is not that into school and he sees "honors kids" as "stuck-up." He and James are neighbors and he was dating Amy at the time of our interview, but they broke up shortly afterward. His parents give him plenty of freedom to do as he wishes. SNS: MySpace. 
Mickey (15, Los Angeles): $10^{\text {th }}$ grade, Mexican, male.

Interview: January 12, 2007.

Mickey's favorite band is My Chemical Romance and his fashion and long bangs reinforce his participation in emo culture. Created by a friend, his profile is filled with photos of the band. Mickey is dating a girl and they talk on the phone constantly, much to the frustration of his friend Zero. Mickey does not connect with most of his peers, although he has a few close friends. Mickey is not doing that well in school, much to his parents' frustration. He speaks Spanish at home and regularly struggles with his parents' restrictions. SNS: MySpace.

\section{Milo (15, Los Angeles): $10^{\text {th }}$ grade, Egyptian, male.}

Interview: January 27, 2007.

Milo tells me that he lives in the "ghetto" with his brother and parents. At home, they speak Arabic and Milo has cousins who live nearby with whom he is quite close. Milo is passionate about gaming and spends a lot of time on World of Warcraft. He daily uses many forms of social media and has tried out a wide variety of social network sites (BlackPlanet, Tagged, Xanga, LiveJournal), but he now sticks to MySpace. He has a girlfriend with whom he tries to spend as much time as possible. SNS: MySpace. 
Myra (15, Iowa): $9^{\text {th }}$ grade, white, female.

Interview: April 22, 2007.

Myra is involved in many activities, mostly because her mother signs her up for everything; she identifies only one of those activities as being her choice. She also goes every weekend to church, where she takes part in more activities. She feels tired and stretched thin from being involved in too many things, but she also accepts her mother's control of her life as unchangeable. Myra is quiet and a bit geeky. She and Leigh are good friends from school, but because of Myra's schedule, they can never get together. SNS: None due to parental restrictions.

Nadine (16, New Jersey): grade unknown, white, female. MySpace: July 12, 2006.

Nadine's MySpace suggests that she is into emo music and generally frustrated with her peers. Her quotes are pulled from content she wrote on MySpace. SNS: MySpace.

Natalie (15, Seattle): $9^{\text {th }}$ grade, white, female.

Interview: January 20, 2007.

Natalie attends church every weekend and participates in youth group every week. Christianity also plays a dominant role in her self-description on MySpace. She plays soccer and will run track in the spring. She is best friends with Ann and 
sleeps over at her house every weekend. She babysits for extra money. Natalie is involved in numerous after-school activities—she dances but does not like it; she is apathetic about playing the oboe; she loves the different sports she engages in. Mostly, she participates in activities to be with friends. SNS: MySpace.

\section{Nicholas (16, Kansas): $10^{\text {th }}$ grade, white, male.}

Interview: April 14, 2007.

Nicholas is extremely active in sports, primarily soccer. He wants to earn a scholarship to go to college and thinks that it might be "neat" to be a nurse because the job would help people and pay well. He thinks he will go into the military like his father, partially because it will help pay for college, but also because it is a good thing to do. He is mostly concerned with hanging out with his friends and goofing off, although he also believes in getting good grades because this allows him to do what he wants on weekends, except when his parents find out that he has been drinking. Nicholas lives with his parents and younger brother. SNS: Facebook, MySpace. 
Nick (16, Los Angeles): $11^{\text {th }}$ grade, black with Native American roots, ${ }^{16}$ male.

Interview: January 12, 2007.

Nick is Denzel's fraternal twin brother. His family is very close and his father has a MySpace and is the football coach at school. Like his brother, he loves his Sidekick and uses it to IM with friends. Nick is an excellent student, passionate about school, and active on the football team. He wants to go to Stanford to become a cancer researcher. He has an 11-year-old sister of whom he and his brother are very protective. SNS: MySpace.

Nora (18, Virginia): grade unknown, white, female.

Blog: September 24, 2006.

Nora loves music, primarily that which she identifies as "pop punk." Her MySpace profile is littered with pictures of various indie rock bands and she talks about going to concerts. She also has photo displays of the TV show Heroes and the Twilight book series. She is short, has long reddish-blond hair, and is heavyset. SNS: MySpace.

\footnotetext{
${ }^{16}$ Nick self-identifies as black with Native American roots; his twin brother Denzel self-identifies as black.
} 


\section{Penelope (15, Nebraska): $9^{\text {th }}$ grade, white, female.}

Interview: April 17, 2007.

Penelope is a socially awkward teen who is conscientious of her lack of popularity at school. She is about to transition schools and is a bit nervous about making friends. She is simultaneously trying to fit in and be unique. She is involved in many activities and her parents keep her schedule rather booked; she echoes her father's view that "only the boring are bored." She does Weight Watchers with her mother, although she is more big boned than overweight. She bikes everywhere and is relatively free to do as she wants, when she has time. SNS: Facebook, MySpace, Xanga.

\section{Red (17, Iowa): $12^{\text {th }}$ grade, white, female.}

Interview: April 18, 2007.

Red is quieter than her friends, but she is also quite emotionally strong. She has had a tough relationship with her family_her father lives 90 minutes away and her mother goes through boyfriends rapidly. Red's source of stability is her boyfriend. He is out of high school and they have been dating for a long time. SNS: Facebook, MySpace. 


\section{Sabrina (14, Texas): $9^{\text {th }}$ grade, white, female.}

Interview: March 15, 2007.

Sabrina is a bit tomboyish, passionate about ROTC, and identifies as a "gaming freak." She is into anime, Harry Potter, and a bit of fandom. She is not particularly popular; she has a small number of friends at school, but none live nearby. Her best friend is male and into video games. Her parents have moved regularly and she has lived in eight places, including Japan and most recently Florida. She uses various social media to keep in touch with old friends. She is close with her parents and they live in a new middle-class suburb. SNS: Deviant Art, MySpace.

\section{Sam (17, Iowa): $11^{\text {th }}$ grade, white, male.}

Interview: April 21, 2007.

He lives with his mother, stepfather, and younger half brother (7). His brother worships him and he wants to go to college nearby so as to not be far away. Sam does not get along with his father. Sam loves baseball, has a private coach, and is looking to get a scholarship through baseball. He goes to a private Catholic school and has a car, which gives him a lot of mobility. He likes to party and drink; this has gotten him into trouble, but nothing serious. He worries that he will upset his mother and wishes he believed in God. He is mostly interested in girls and socializing and sees himself as a generic teen who just "goes with the flow." SNS:

\section{Facebook.}


Samantha (18, Seattle): $12^{\text {th }}$ grade, white, female.

Interview: January 20, 2007.

Samantha is an attractive, preppy-seeming girl. I get the sense that she is well liked but she also switched schools every year in high school so it may just be that she is personable from constant change. She has a boyfriend she really likes and she is quite religious. Church plays a critical role in her life and her profile speaks extensively about Jesus. SNS: MySpace.

\section{Sara (16, Austin): $10^{\text {th }}$ grade, white, female.}

Interview: March 15, 2007.

Sara comes from a wealthy family and her house is designed to signal their wealth. Her mother has a $\mathrm{PhD}$ and her father is a lawyer. Her parents are swimming fanatics; she prefers track. She has a car and drives her friends around; they primarily hang out at her house. She is popular at school, but she feels stressed and insecure. Although she has a group of friends, her boyfriend is the center of her world and they talk every night. SNS: None due to parental restrictions.

\section{Sasha (16, Michigan): $10^{\text {th }}$ grade, white, female.}

Interview: June 26, 2007.

Sasha's parents are divorced and she splits time with both; she has an older sister who graduated from high school. Her mother lives next door to her best friend, 
Bianca, and she and Bianca regularly get together at Sasha's mother's house. She is a pretty good dancer and enjoys school. She is more outgoing than Bianca, shares her love of text messaging, but she is forbidden to use Facebook. SNS: None due to parental restrictions.

\section{Scott (15, Iowa): $9^{\text {th }}$ grade, white, male.}

Interview: April 20, 2007.

Scott goes to a Catholic school, but he does not really like Catholicism. He identifies himself as politically liberal compared to his conservative peers. Scott plays football and is involved in theater at school. His parents give him plenty of freedom, but he cannot drive, which is a huge limitation. He likes to play sports-related video games. Scott is tall with short brown hair. SNS: Facebook.

\section{Seong (17, Los Angeles): $11^{\text {th }}$ grade, Korean, female.}

Interview: February 20, 2007.

Seong goes to a magnet school for music, although she is more interested in science than in music. She wants to go to college for pharmacy and she volunteered at a pharmacy. She lives with her parents; her older sister is in college. She speaks Korean with her mother, but her father wants to improve his English. Her boyfriend and friends are important to her and she sees them every weekend. She used to hang out with mostly Asian kids but not since she started high school. She sees Asian teens as having a mob mentality and says that many of her interests and activities 
changed when she stopped hanging out with them. SNS: Cyworld, Facebook, MySpace, Xanga.

\section{Shean (17, Los Angeles): $12^{\text {th }}$ grade, black, male.}

Interview: March 5, 2007.

Shean's parents are divorced and he has eight siblings and half siblings. His father lives in Las Vegas and they do not get along, although he and his mother are close. Friends are extremely important to Shean and he spends a lot of time running around with his crew. He has a lot of freedom when it comes to going out, in part because his mother is close with the mothers of his friends and his sister's friends are the younger siblings of his friends. Money is pretty important to him and he would love to be famous. SNS: MySpace.

\section{Skyler Sierra (18, Colorado): $12^{\text {th }}$ grade, white, female.}

Blog: March 16, 2006.

Skyler is the daughter of a well-known technology consultant. Skyler conveys her opinions of technology with her mother, who often quotes her on her blog; the quotes used in my dissertation are from her mother's blog. Skyler has long blond hair and blue eyes. SNS: MySpace. 
Stan (18, Iowa): $12^{\text {th }}$ grade, white, male.

Interview: April 18, 2007.

Stan plays many sports and has signed up to go into the National Guard when he graduates. He lives with his mother in a clearly working-class environment. Wolf now lives with Stan after being kicked out of his house. Stan used to date Cachi, but they were never serious. His friends joke that he is a male slut, and they like to tease him in general, probably because he reacts in an entertaining manner. His family does not have a working computer. SNS: MySpace.

\section{Summer (15, Michigan): between $9^{\text {th }}$ and $10^{\text {th }}$ grade, white, female.}

Interview: June 27, 2007.

Summer is a ballerina, plays volleyball, and identifies as a bookworm. She has a handful of friends, but she is not in the popular "wealthy" crowd at her Catholic school. Money is an issue for her family and she is not able to get a car or phone because of it. In the fifth grade, Summer was bullied by her best friend; she switched schools because of the rumors. She lives with her parents and younger sister. SNS:

MySpace. 


\section{Tara (16, Michigan): between $9^{\text {th }}$ and $10^{\text {th }}$ grade, Vietnamese, female.}

Interview: June 27, 2007.

Tara is extremely bubbly and sociable. She lightens up when talking about friends or shopping. She is involved in music, sports, clubs, and so on, but she is more interested in friends than in school. Lila is her older sister and she has another sister who is much older. She and Lila are very close and they finish each other's sentences, share clothes, and share most interests. She does not have as much freedom as Lila does and this can be frustrating at times. SNS: Facebook, MySpace.

\section{Tom (12, Boston): $7^{\text {th }}$ grade, white, male.}

Berkman Focus Group: November 15, 2007.

Tom is really into technology and a bit of a nerd. He likes to go the Science Museum for fun and is aware of the legality of various Internet practices. He attends a very expensive and elite private school. This interview was conducted as a focus group by the Berkman Center's Digital Natives project who graciously shared Tom's comments with me. SNS: Facebook.

\section{Traviesa (15, Los Angeles): $9^{\text {th }}$ grade, Hispanic, female.}

Interview: December 5, 2006.

Traviesa lives with her older sister, who is extremely strict. Her mother is a meth addict and Traviesa previously had a problem with drugs and "troublemaking" 
crowds. She lives in a gang-ridden community and is trying to find focus and get back on track after years of getting into trouble. In middle school, she identified as a loner but then became friends with the "cool" kids and recently realized that they are not as interesting as she once thought. She is extremely expressive when she talks and makes countless facial expressions to emphasize her points. SNS: MySpace.

\section{Ty (17, Los Angeles): $12^{\text {th }}$ grade, black, male.}

Interview: February 20, 2007.

Ty lives in a group home and is "in the system" (a.k.a. foster care). He used to live in Philadelphia with his mother before moving to L.A. to live with his father, but he got kicked out. The group home has some strict rules, including limitations on mobility and restrictions for visiting friends. He does not have a cell phone and there is no computer access at the group home, but he gets online at school. He draws a lot, loves salsa, and makes his own music with a guy who is trying to keep him out of trouble. He is into girls and misses being able to go to parties and hang out with friends. He has three siblings - they are all living in different places. SNS: MySpace.

\section{Wolf (18, Iowa): $12^{\text {th }}$ grade, white, male.}

Interview: April 18, 2007.

Wolf spends the entire interview interrupting his friends and raging about everything. He is tremendously angry, but it is clear that this is a cover for some 
major issues. He drinks to excess and engages in a lot of violent behavior. He comes from a working-class background. He loves his mother but they fight a lot. He and his alcoholic father fight more. He is now living with Stan after having been kicked out of his own house. He plans to join the military when he graduates. He is very condescending toward Cachi (who laughs at him) and Red (who takes it personally). SNS: MySpace.

\section{Zero (15, Los Angeles): $10^{\text {th }}$ grade, black with Native American roots, male.}

Interview: January 12, 2007.

Zero and Mickey are friends in a racially segregated school where there are few connections between black and Mexican teens. Zero is overweight and his description of his life suggests that he has few friends. Outside of school, Zero often helps his father with his housecleaning business. His father has a strong work ethic and believes that Zero should work to earn his own money. He and his father are close. Zero is into video games and uses the Internet mostly to shop, play games, and search for information. To talk with Mickey, Zero uses the phone. SNS: MySpace (but he rarely uses it). 


\section{Appendix 2: Features and Functionality}

The social network sites MySpace and Facebook have a variety of different features that teens use as a part of their participation on the sites. The structure of these sites is driven by their genre and its history (see boyd and Ellison 2007). My analysis, discussion, and use of teen quotes assume familiarity with these features and their functionality. This Appendix is intended to provide a brief overview of the features for those who are unfamiliar with the sites. What I describe here reflects the features and functionality that were present at the time of my fieldwork in 20042007; many of these features have changed since then. Unless indicated otherwise, the features that I describe are available on both sites.

While teens are not the only participants on social network sites, I am focusing on the features that are most relevant to teens. I do not document all features available, but rather, I focus on the ones that are most significant to my dissertation. The features themselves say little about the complexity of how teens use them and alter their practices because of them. Yet a cursory understanding of the most prevalent features is tremendously helpful for understanding the analysis presented throughout this dissertation.

Social network sites are web-based services that allow individuals to (1) construct a public or semi-public profile within a bounded system, (2) publicly articulate a list of other users ("Friends") with whom they share a connection, and (3) view and 
traverse their list of connections and those made by others within the system. During my fieldwork, MySpace and Facebook profiles comprised three core sections: (1) the content users provide to craft their profiles, (2) the list of Friends, and (3) a section for comments. More elements have been added since.

When teens join a social network site, they are asked to upload a photograph and fill out a set of forms that request demographic information (e.g., birthday, sex, location, etc.), taste preferences (e.g., favorite music/TV/movies, sexual orientation, interests, etc.). MySpace and Facebook ask different specific questions, but they generally fit into these categories. MySpace also requests responses to open-ended fields like "About Me" and "Who I'd Like to Meet." Teens are encouraged to describe themselves through free-form text although, as discussed in Chapter 4, teens do not always respond to these elements directly.

The content teens provide is used to automatically generate a Profile. Participants can alter their answers at any point; editing a profile also provides additional questions that were not available during the signup process.

When people sign up to Facebook, they are also asked to choose a Network. There were four types of networks: college, high school, corporate, and region. Teens could add themselves to their high-school network and/or their region's network. Anyone can be a member of a regional network, but individuals can be part of only one regional network and cannot change their regional network more than once a day. When teens sign up to join a high-school network, their request is sent to the 
network's moderators for approval. Teens who have access to .edu email addresses can also join college-level networks.

While profiles are automatically generated, they can be modified in various ways. On MySpace, users can insert HTML, Javascript, and CSS Code into some of the profile fields (most notably, the "About Me" one) to alter the background and layout, change the font colors and size, add multimedia, and modify or hide other elements on the profile. Teens talk about the alteration of these profiles as Layouts, Backgrounds, and, occasionally, Glitter. Although most MySpace participants do not know how to create this code, they can Copy/Paste this content from a variety of free websites where individuals (and marketers, spammers, and scammers) have provided code for MySpace layouts. This "feature" was not designed by MySpace; rather, it was a loophole that users figured out how to exploit and that MySpace did not stop. Teens' familiarity with this code is one form of technological literacy (Perkel 2006).

Facebook's profiles are structured as modules. Users can move modules around to rearrange their pages, but they cannot change the visual look and feel. At the end of my fieldwork, teens could also add modules created by third parties and vetted by Facebook called Apps (or applications). The thousands of available Apps are quite diverse. Examples of Apps that were popular when I was writing include: (fluff)Friends, Scrabulous, Fun Wall, and Movies. (fluff)Friends allows users to add a pet to their profile that can be fed and loved by Friends through clicks. Scrabulous 
allows users to play each other in an asynchronous word game that closely resembles Scrabble. Fun Wall resembles the Facebook Wall feature, only it allows users to add graffiti, images, and other multimedia. Movies allows users to list favorite movies and compare their tastes with Friends. Apps were introduced toward the end of my fieldwork so few teens talk about them, but they have become increasingly popular. MySpace has since added Apps to its site as well.

After creating a profile, teens are encouraged to search for people they know or invite their friends to the site. They may then "add" these people as Friends. Such an act prompts a Friend request that requires approval for the Friend-ship to be confirmed. Upon approval, both parties are listed as Friends on the site. On Facebook, teens are invited to describe their relationship to those whom they indicate as Friends. While this feature was designed with the idea that people would add only their closest and dearest, teens have a variety of different incentives for adding people as Friends (boyd 2006b). Since Friends are not always the same as friends, I capitalize social network site Friends to differentiate the usage.

Social network sites publicly display Friend connections on teens' profiles. This allows visitors to the profile to peruse a teen's Friends. Facebook displays six random Friends from a teen's primary network on the profile, allowing visitors to click to see more. MySpace allows users to choose their Top Friends. Top Friends, originally called Top 8, is a feature in which teens can order their Friends for public display. Originally, teens could pick only eight Friends to display; they can now choose up to 
24. Facebook does not natively have a Top Friends feature, but this is a popular App. Facebook does allow users to indicate one of their Friends as their significant other or spouse; this is displayed separately, alongside the user's tastes and demographic information. Some teens use this field to indicate their best friend, so when a teen's profile says, "Elizabeth is married to Hannah," it is not necessarily an indicator that the two teens have same-sex interests.

Profiles include a section where Friends can leave comments on each other's profiles. On MySpace, this feature is called Comments; on Facebook, this is called the Wall. The messages that people leave on a person's profile are visible to anyone who can view that profile. Conversations often take place here as teens respond to comments on their profile by leaving a comment on the commenter's profile.

MySpace has a section on the profile called Headlines where teens can post a pithy message that is displayed at the top of their profile; headlines are also visible in searches on the site. At the top of Facebook profiles is a feature called Status Updates. Teens are prompted to answer the question "What are you doing right now?" The answer appears under a person's name. Thus, if I answered, “is writing," my profile would say, "danah boyd is writing." Teens can see all of their Friends' status updates on a single page and they can subscribe to these updates via RSS. Updates disappear after a few days.

Both MySpace and Facebook profiles support a wide array of media. MySpace allows teens to add their favorite song to their profile. By default, when someone 
views a profile with a song on it, the song starts automatically. MySpace users can also upload videos to their profile; these can be displayed on a teen's profile or accessed through their collection of videos. Teens can leave comments on their Friends' videos. Both sites support the uploading of photos. Teens can organize their photos into albums and their Friends can comment on the photos.

Teens can send private messages to each other through each site, but not across sites. These resemble web mail and teens have an inbox available to them when they $\log$ in. MySpace also has a feature called Bulletins. Teens can post a message here and this message then appears on all of their Friends' bulletin boards. When teens check their bulletin boards, they can see all of the messages that all of their Friends posted.

Both sites support Groups. Participants can join public groups and communicate with one another using the group's bulletin board features. Teens' groups are listed on their profiles. Many teens join groups to have them listed on their profiles and never visit the actual group page. While there are groups that connect people around a topic, many of the groups have goofy names like "When I was your age, Pluto was a planet."

Midway through my fieldwork, Facebook introduced a feature called News Feed. When teens log in, their homepage consists of a list of automatically generated updates about their Friends' activity on the site. This constitutes the News Feed. Content on the News Feed includes things like who added whom as Friends, who updated their profile, who added new photos, and who broke up with whom. The 
News Feed originally had no advertisements, but this quickly changed. Initially, the News Feed was highly controversial, in part because teens could not adjust what appeared there (boyd 2008a). Facebook responded to the controversy by offering privacy settings for this feature so that teens can control what content of theirs might appear there. Teens' profiles also contain a Mini-Feed that is simply a list of updates about that person. Presumably, those are the elements that are broadcast to that teen's Friends' News Feed.

MySpace and Facebook take entirely different approaches to privacy. MySpace's approach to privacy is simple. Profiles are either public or private. Public profiles can be accessed by anyone on the site. Private profiles are visible only to a teen's Friends. MySpace users also have controls that allow them to limit who can contact them. Initially, private profiles were available only to those under 16, but MySpace eventually made this feature available to everyone. Teens can ban non-Friends from sending messages; block bands, comedians, and filmmakers from sending them Friend requests; and require those who do add them as Friends to indicate their last name or email.

Facebook's approach to privacy is far more complex than MySpace's. By default, a teen's profile is visible to their Friends and anyone in any of their networks. Through privacy settings, teens can adjust each component of their profile, making specific elements visible to "All of my networks and all of my friends," "Some of 


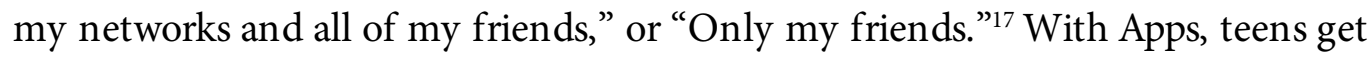
two additional options: "Only me" and "No one." The first three options and "Everyone" are options for visibility in Facebook searches. There is an additional toggle button for making the profile visible to search engines; this is on by default. More privacy options allow teens to control who can contact them, who can see their primary photo, how much of their data is used by partner advertisers, and which of their updates are broadcast to Friends' News Feed. There was also a Limited Profile option that allowed participants to add others as Friends but then allow them to see only limited profile modules. Participants may also block specific people from specific content. Facebook continues to add options to its privacy settings and much has changed since I did my fieldwork. For example, Facebook now allows teens to group their Friends into groups and organize them accordingly. While Facebook gives users complete flexibility over who can access what, it does so at the expense of simplicity and clarity.

Both Facebook and MySpace have tools that allow users to search for their friends, peers, and colleagues. Facebook's simple search feature allows teens to search by name and narrow by network; users can also surf Facebook through the list of Friends. MySpace provides three ways of finding people: search, browse, and surf. Anyone can search for anyone, provided that the searcher knows the other person's full name or email address. Adults may browse only for other adults, aged 18-68.

\footnotetext{
${ }^{17}$ Note: After my data collection, Facebook added an additional option so that participants could make their profiles visible to friends of friends.
} 
(Because teenagers regularly listed themselves as 69+, MySpace stopped allowing anyone to search for users 69 and older.) Minors may browse for anyone who is over 16. Those who are 14 or 15 are not visible through the browsing feature to anyone, regardless of age. On both Facebook and MySpace, surfing is the dominant way in which people find each other. Teens view the Friends lists of their Friends and surf to their profiles that way.

Both sites have many more features and the features continue to change. This description is by no means exhaustive, although the features that I lay out here play a significant role in my data, analysis, and discussion. 


\section{Appendix 3: Creative Commons License}

This is the text of Creative Commons' Attribution-NonCommercial-NoDerivs License, version 3.0. ${ }^{18}$

\section{License}

THE WORK (AS DEFINED BELOW) IS PROVIDED UNDER THE TERMS OF THIS CREATIVE COMMONS PUBLIC LICENSE ("CCPL" OR "LICENSE"). THE WORK IS PROTECTED BY COPYRIGHT AND/OR OTHER APPLICABLE LAW. ANY USE OF THE WORK OTHER THAN AS AUTHORIZED UNDER THIS LICENSE OR COPYRIGHT LAW IS PROHIBITED.

BY EXERCISING ANY RIGHTS TO THE WORK PROVIDED HERE, YOU ACCEPT AND AGREE TO BE BOUND BY THE TERMS OF THIS LICENSE. TO THE EXTENT THIS LICENSE MAY BE CONSIDERED TO BE A CONTRACT, THE LICENSOR GRANTS YOU THE RIGHTS CONTAINED HERE IN CONSIDERATION OF YOUR ACCEPTANCE OF SUCH TERMS AND CONDITIONS.

\section{Definitions}

1. "Collective Work" means a work, such as a periodical issue, anthology or encyclopedia, in which the Work in its entirety in unmodified form, along with one

\footnotetext{
${ }^{18}$ See: http://creativecommons.org/licenses/by-nc-nd/3.0/us/
} 
or more other contributions, constituting separate and independent works in themselves, are assembled into a collective whole. A work that constitutes a Collective Work will not be considered a Derivative Work (as defined below) for the purposes of this License.

2. "Derivative Work" means a work based upon the Work or upon the Work and other pre-existing works, such as a translation, musical arrangement, dramatization, fictionalization, motion picture version, sound recording, art reproduction, abridgment, condensation, or any other form in which the Work may be recast, transformed, or adapted, except that a work that constitutes a Collective Work will not be considered a Derivative Work for the purpose of this License. For the avoidance of doubt, where the Work is a musical composition or sound recording, the synchronization of the Work in timed-relation with a moving image ("synching") will be considered a Derivative Work for the purpose of this License.

3. "Licensor" means the individual, individuals, entity or entities that offers the Work under the terms of this License.

4. "Original Author" means the individual, individuals, entity or entities who created the Work.

5. "Work" means the copyrightable work of authorship offered under the terms of this License. 
6. "You" means an individual or entity exercising rights under this License who has not previously violated the terms of this License with respect to the Work, or who has received express permission from the Licensor to exercise rights under this License despite a previous violation.

\section{Fair Use Rights.}

Nothing in this license is intended to reduce, limit, or restrict any rights arising from fair use, first sale or other limitations on the exclusive rights of the copyright owner under copyright law or other applicable laws.

\section{License Grant.}

Subject to the terms and conditions of this License, Licensor hereby grants You a worldwide, royalty-free, non-exclusive, perpetual (for the duration of the applicable copyright) license to exercise the rights in the Work as stated below:

1. to reproduce the Work, to incorporate the Work into one or more Collective Works, and to reproduce the Work as incorporated in the Collective Works; and,

2. to distribute copies or phonorecords of, display publicly, perform publicly, and perform publicly by means of a digital audio transmission the Work including as incorporated in Collective Works.

The above rights may be exercised in all media and formats whether now known or hereafter devised. The above rights include the right to make such modifications 
as are technically necessary to exercise the rights in other media and formats, but otherwise you have no rights to make Derivative Works. All rights not expressly granted by Licensor are hereby reserved, including but not limited to the rights set forth in Sections 4(d) and 4(e).

\section{Restrictions.}

The license granted in Section 3 above is expressly made subject to and limited by the following restrictions:

1. You may distribute, publicly display, publicly perform, or publicly digitally perform the Work only under the terms of this License, and You must include a copy of, or the Uniform Resource Identifier for, this License with every copy or phonorecord of the Work You distribute, publicly display, publicly perform, or publicly digitally perform. You may not offer or impose any terms on the Work that restrict the terms of this License or the ability of a recipient of the Work to exercise the rights granted to that recipient under the terms of the License. You may not sublicense the Work. You must keep intact all notices that refer to this License and to the disclaimer of warranties. When You distribute, publicly display, publicly perform, or publicly digitally perform the Work, You may not impose any technological measures on the Work that restrict the ability of a recipient of the Work from You to exercise the rights granted to that recipient under the terms of the License. This Section 4(a) applies to the Work as incorporated in a Collective Work, but this does not require the Collective Work apart from the Work itself to 
be made subject to the terms of this License. If You create a Collective Work, upon notice from any Licensor You must, to the extent practicable, remove from the Collective Work any credit as required by Section 4(c), as requested.

2. You may not exercise any of the rights granted to You in Section 3 above in any manner that is primarily intended for or directed toward commercial advantage or private monetary compensation. The exchange of the Work for other copyrighted works by means of digital file-sharing or otherwise shall not be considered to be intended for or directed toward commercial advantage or private monetary compensation, provided there is no payment of any monetary compensation in connection with the exchange of copyrighted works.

3. If You distribute, publicly display, publicly perform, or publicly digitally perform the Work (as defined in Section 1 above) or Collective Works (as defined in Section 1 above), You must, unless a request has been made pursuant to Section 4(a), keep intact all copyright notices for the Work and provide, reasonable to the medium or means You are utilizing: (i) the name of the Original Author (or pseudonym, if applicable) if supplied, and/or (ii) if the Original Author and/or Licensor designate another party or parties (e.g. a sponsor institute, publishing entity, journal) for attribution ("Attribution Parties") in Licensor's copyright notice, terms of service or by other reasonable means, the name of such party or parties; the title of the Work if supplied; to the extent reasonably practicable, the Uniform Resource Identifier, if any, that Licensor specifies to be associated with the Work, 
unless such URI does not refer to the copyright notice or licensing information for the Work. The credit required by this Section 4(c) may be implemented in any reasonable manner; provided, however, that in the case of a Collective Work, at a minimum such credit will appear, if a credit for all contributing authors of the Collective Work appears, then as part of these credits and in a manner at least as prominent as the credits for the other contributing authors. For the avoidance of doubt, You may only use the credit required by this clause for the purpose of attribution in the manner set out above and, by exercising Your rights under this License, You may not implicitly or explicitly assert or imply any connection with, sponsorship or endorsement by the Original Author, Licensor and/or Attribution Parties, as appropriate, of You or Your use of the Work, without the separate, express prior written permission of the Original Author, Licensor and/or Attribution Parties.

4. For the avoidance of doubt, where the Work is a musical composition:

a. Performance Royalties Under Blanket Licenses. Licensor reserves the exclusive right to collect whether individually or, in the event that Licensor is a member of a performance rights society (e.g. ASCAP, BMI, SESAC), via that society, royalties for the public performance or public digital performance (e.g. webcast) of the Work if that performance is primarily intended for or directed toward commercial advantage or private monetary compensation. 
b. Mechanical Rights and Statutory Royalties. Licensor reserves the exclusive right to collect, whether individually or via a music rights agency or designated agent (e.g. Harry Fox Agency), royalties for any phonorecord You create from the Work ("cover version") and distribute, subject to the compulsory license created by 17 USC Section 115 of the US Copyright Act (or the equivalent in other jurisdictions), if Your distribution of such cover version is primarily intended for or directed toward commercial advantage or private monetary compensation.

5. Webcasting Rights and Statutory Royalties. For the avoidance of doubt, where the Work is a sound recording, Licensor reserves the exclusive right to collect, whether individually or via a performance-rights society (e.g. SoundExchange), royalties for the public digital performance (e.g. webcast) of the Work, subject to the compulsory license created by 17 USC Section 114 of the US Copyright Act (or the equivalent in other jurisdictions), if Your public digital performance is primarily intended for or directed toward commercial advantage or private monetary compensation.

\section{Representations, Warranties and Disclaimer}

UNLESS OTHERWISE MUTUALLY AGREED TO BY THE PARTIES IN

WRITING, LICENSOR OFFERS THE WORK AS-IS AND ONLY TO THE

EXTENT OF ANY RIGHTS HELD IN THE LICENSED WORK BY THE

LICENSOR. THE LICENSOR MAKES NO REPRESENTATIONS OR

WARRANTIES OF ANY KIND CONCERNING THE WORK, EXPRESS, 
IMPLIED, STATUTORY OR OTHERWISE, INCLUDING, WITHOUT

LIMITATION, WARRANTIES OF TITLE, MARKETABILITY,

MERCHANTIBILITY, FITNESS FOR A PARTICULAR PURPOSE,

NONINFRINGEMENT, OR THE ABSENCE OF LATENT OR OTHER DEFECTS, ACCURACY, OR THE PRESENCE OF ABSENCE OF ERRORS, WHETHER OR

NOT DISCOVERABLE. SOME JURISDICTIONS DO NOT ALLOW THE

EXCLUSION OF IMPLIED WARRANTIES, SO SUCH EXCLUSION MAY NOT APPLY TO YOU.

\section{Limitation on Liability.}

EXCEPT TO THE EXTENT REQUIRED BY APPLICABLE LAW, IN NO EVENT WILL LICENSOR BE LIABLE TO YOU ON ANY LEGAL THEORY FOR ANY SPECIAL, INCIDENTAL, CONSEQUENTIAL, PUNITIVE OR EXEMPLARY DAMAGES ARISING OUT OF THIS LICENSE OR THE USE OF THE WORK, EVEN IF LICENSOR HAS BEEN ADVISED OF THE POSSIBILITY OF SUCH DAMAGES.

\section{Termination}

1. This License and the rights granted hereunder will terminate automatically upon any breach by You of the terms of this License. Individuals or entities who have received Collective Works (as defined in Section 1 above) from You under this License, however, will not have their licenses terminated provided such individuals 
or entities remain in full compliance with those licenses. Sections $1,2,5,6,7$, and 8 will survive any termination of this License.

2. Subject to the above terms and conditions, the license granted here is perpetual (for the duration of the applicable copyright in the Work).

Notwithstanding the above, Licensor reserves the right to release the Work under different license terms or to stop distributing the Work at any time; provided, however that any such election will not serve to withdraw this License (or any other license that has been, or is required to be, granted under the terms of this License), and this License will continue in full force and effect unless terminated as stated above.

\section{Miscellaneous}

1. Each time You distribute or publicly digitally perform the Work (as defined in Section 1 above) or a Collective Work (as defined in Section 1 above), the Licensor offers to the recipient a license to the Work on the same terms and conditions as the license granted to You under this License.

2. If any provision of this License is invalid or unenforceable under applicable law, it shall not affect the validity or enforceability of the remainder of the terms of this License, and without further action by the parties to this agreement, such provision shall be reformed to the minimum extent necessary to make such provision valid and enforceable. 
3. No term or provision of this License shall be deemed waived and no breach consented to unless such waiver or consent shall be in writing and signed by the party to be charged with such waiver or consent.

4. This License constitutes the entire agreement between the parties with respect to the Work licensed here. There are no understandings, agreements or representations with respect to the Work not specified here. Licensor shall not be bound by any additional provisions that may appear in any communication from You. This License may not be modified without the mutual written agreement of the Licensor and You.

\section{Creative Commons Notice}

Creative Commons is not a party to this License, and makes no warranty whatsoever in connection with the Work. Creative Commons will not be liable to You or any party on any legal theory for any damages whatsoever, including without limitation any general, special, incidental or consequential damages arising in connection to this license. Notwithstanding the foregoing two (2) sentences, if Creative Commons has expressly identified itself as the Licensor hereunder, it shall have all rights and obligations of Licensor.

Except for the limited purpose of indicating to the public that the Work is licensed under the CCPL, Creative Commons does not authorize the use by either party of the trademark "Creative Commons" or any related trademark or logo of Creative Commons without the prior written consent of Creative Commons. Any 
permitted use will be in compliance with Creative Commons' then-current trademark usage guidelines, as may be published on its website or otherwise made available upon request from time to time. For the avoidance of doubt, this trademark restriction does not form part of this License.

Creative Commons may be contacted at http://creativecommons.org/. 